Supporting Information for

\title{
Thiopyrylium Scaffolds from the Lewis/Brønsted-acid-promoted Cyclization of Thioethers
}

Noriyoshi Nagahora, ${ }^{*}$ Rina Tanaka, Tomoyo Tada, Akira Yasuda, Yuji Yamada, Kosei Shioji, and Kentaro Okuma

Department of Chemistry, Faculty of Science, Fukuoka University, Jonan-ku, Fukuoka 814-0180, Japan

E-mail: nagahora@fukuoka-u.ac.jp

Table of Contents

$\begin{array}{ll}\text { Experimental Detail } & \text { S2-S35 }\end{array}$

$\begin{array}{ll}\text { Theoretical Calculations } & \text { S36-S46 }\end{array}$

$\begin{array}{ll}\text { References } & \text { S47 }\end{array}$

${ }^{1} \mathrm{H}$ and ${ }^{13} \mathrm{C}$ NMR Spectra of All New Compounds $\quad$ S48-S129 


\section{Experimental Detail}

General. All solvents were purified by standard methods. Preparative thin-layer chromatography (PTLC) was performed on Merck silica gel 60 PF254. Column chromatography was performed on silica gel 60N (Kanto Chemical) under an ambient atmosphere. ${ }^{1} \mathrm{H}(400 \mathrm{MHz})$ and ${ }^{13} \mathrm{C}$ NMR $(101 \mathrm{~Hz})$ spectra were recorded in $\mathrm{CDCl}_{3}$ on a Bruker Avance spectrometer using the residual resonances of $\mathrm{CHCl}_{3}\left(\delta_{\mathrm{H}}=7.26\right)$ and $\mathrm{CDCl}_{3}$ $\left(\delta_{\mathrm{C}}=77.0\right)$ as well as of $\mathrm{CD}_{3} \mathrm{CN}\left(\delta_{\mathrm{H}}=1.94 ; \delta_{\mathrm{C}}=1.32\right)$ as the internal standards to reference the ${ }^{1} \mathrm{H}$ and ${ }^{13} \mathrm{C}$ NMR spectra. ${ }^{19} \mathrm{~F}$ NMR spectra (376 MHz) were recorded on a Bruker Avance spectrometer using $\mathrm{CFCl}_{3}\left(\delta_{\mathrm{F}}=0.00\right)$ as an external standard. The assignment of the signals was typically accomplished on the basis of 1D (homodecoupling and DEPT) and 2D (COSY, HMQC, and HMBC) NMR techniques. Unless otherwise stated, all ${ }^{13} \mathrm{C}$ and ${ }^{19} \mathrm{~F} \mathrm{NMR}$ experiments were performed using broad-band ${ }^{1} \mathrm{H}$ decoupling. EI and ESI-TOF mass spectral data were obtained on a JEOL JMS-GCmateII and a JEOL JMS-T100CS spectrometer, respectively. Absorption spectra were recorded on a JASCO V-550 UV/Vis or a Shimadzu UV-3600 UV/vis/NIR spectrometer. Elemental analyses were carried out on a JM11 CHN analyzer by J-Science Lab. All melting points were determined on a Yanaco micro-melting point apparatus or a Mettler Toledo MP90 melting point system, and are uncorrected.

Materials. Unless stated otherwise, all materials were purchased from common commercial chemical suppliers and used without further purification unless stated otherwise. All reactions were carried out under an inert atmosphere of argon or nitrogen. 5-tert-Butyl-2-sulfanylbenzaldehyde was prepared according to the reported procedure. ${ }^{1}$

\section{Synthesis of 4-(tert-Butyl)-2-(5,5-dimethyl-1,3-dioxan-2-yl)benzenethiol}

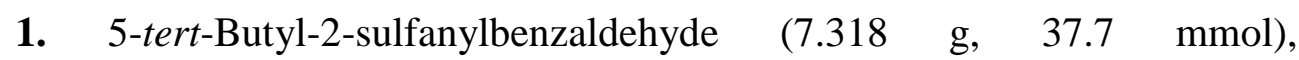
2,2-dimethylpropan-1,3-diol (3.954 g, $38.0 \mathrm{mmol})$, and $p$-toluenesulfonic acid monohydrate $(0.059 \mathrm{~g}, 0.34 \mathrm{mmol})$ were dissolved in toluene $(70 \mathrm{~mL})$.

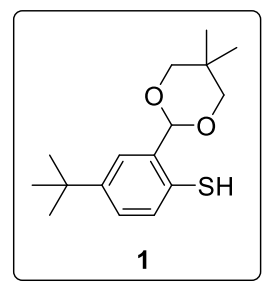
After being stirred at reflux for $4 \mathrm{~h}$ using an oil bath, the reaction mixture was allowed to cool to room temperature. After the solvent was removed under reduced pressure, purification of the crude product by column chromatography on silica gel (elution with dichloromethane) afforded 4-(tert-butyl)-2-(5,5-dimethyl-1,3-dioxan-2-yl)benzenethiol 1 (7.063 g, 25.2 mmol, 
$67 \%)$ as yellow oil. 1: ${ }^{1} \mathrm{H} \mathrm{MNR}\left(400 \mathrm{MHz}, \mathrm{CDCl}_{3}\right) \delta 7.67(\mathrm{~d}, J=2.4 \mathrm{~Hz}, 1 \mathrm{H}, \mathrm{ArH}), 7.25(\mathrm{~d}, J$ $=8.2 \mathrm{~Hz}, 1 \mathrm{H}, \mathrm{ArH}), 7.21(\mathrm{dd}, J=2.4,8.2 \mathrm{~Hz}, 1 \mathrm{H}, \mathrm{ArH}), 5.56(\mathrm{~s}, 1 \mathrm{H}, \mathrm{CH}), 3.78(\mathrm{~d}, J=10.8$ $\left.\mathrm{Hz}, 2 \mathrm{H}, \mathrm{CH}_{2}\right), 3.69$ (d, J = 10.8 Hz, 2H, $\left.\mathrm{CH}_{2}\right), 3.57$ (s, 1H, SH), 1.31 (s, 3H, $\left.\mathrm{CH}_{3}\right), 1.31$ (s, 9H, $\left.\mathrm{CH}_{3}\right), 0.80\left(\mathrm{~s}, 3 \mathrm{H}, \mathrm{CH}_{3}\right) ;{ }^{13} \mathrm{C}\left\{{ }^{1} \mathrm{H}\right\}$ NMR (101 MHz, $\left.\mathrm{CDCl}_{3}\right) \delta 149.4$ (C), 136.2 (C), 131.4 $(\mathrm{CH}), 126.3(\mathrm{CH}), 126.0(\mathrm{C}), 123.6(\mathrm{CH}), 100.2(\mathrm{CH}), 77.7\left(\mathrm{CH}_{2}\right), 34.5(\mathrm{C}), 31.2\left(\mathrm{CH}_{3}\right), 30.2$ (C), $23.2\left(\mathrm{CH}_{3}\right), 21.8\left(\mathrm{CH}_{3}\right)$; MS (EI, positive mode): $\mathrm{m} / \mathrm{z} 280\left([\mathrm{M}]^{+}\right)$; Anal. Calcd for $\mathrm{C}_{16} \mathrm{H}_{24} \mathrm{O}_{2} \mathrm{~S}: \mathrm{C}, 68.53 ; \mathrm{H}, 8.63 \%$; found: $\mathrm{C}, 68.22 ; \mathrm{H}, 8.69 \%$.

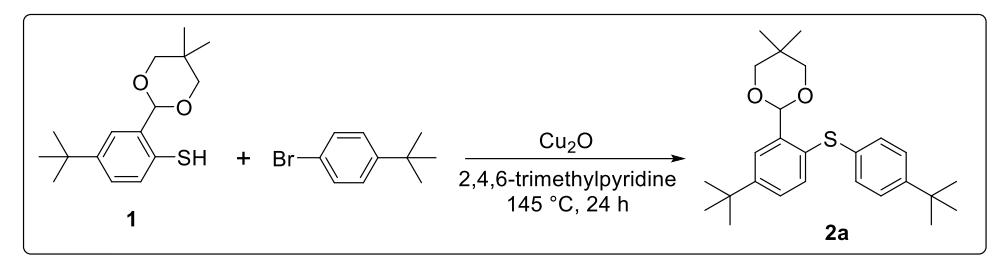

Synthesis of 2-[5-tert-Butyl-2-(4-tert-butylphenylthio)phenyl]-5,5-dimethyl-1,3-dioxane 2a. Benzenethiol 1 (378 mg, $1.35 \mathrm{mmol}$ ), 1-bromo-4-tert-butylbenzene (353 mg, $1.66 \mathrm{mmol}$ ), and copper(I) oxide (386 mg, $2.70 \mathrm{mmol}$ ) were dissolved in 2,4,6-trimethylpyridine (6 mL). After being stirred at $145^{\circ} \mathrm{C}$ for $24 \mathrm{~h}$ using an oil bath, the reaction mixture was allowed to cool to room temperature. After the solvent was removed under reduced pressure, purification of the crude product by column chromatography on silica gel (eluent: hexane/dichloromethane $\quad=\quad 1 / 1, \quad \mathrm{v} / \mathrm{v}) \quad$ afforded 2-[5-tert-butyl-2-(4-tert-butylphenylthio)phenyl]-5,5-dimethyl-1,3-dioxane 2a (325 mg, 0.788 mmol, 58\%) as colorless solid. 2a: mp 139-140 ${ }^{\circ} \mathrm{C} ;{ }^{1} \mathrm{H}$ NMR $\left(400 \mathrm{MHz}, \mathrm{CDCl}_{3}\right) \delta 7.78(\mathrm{~d}, J$ $=2.0 \mathrm{~Hz}, 1 \mathrm{H}, \mathrm{ArH}), 7.29(\mathrm{~d}, J=8.4 \mathrm{~Hz}, 1 \mathrm{H}, \mathrm{ArH}), 7.29$ (d, $J=8.4 \mathrm{~Hz}, 2 \mathrm{H}, \mathrm{ArH}), 7.21(\mathrm{dd}, J$ $=8.4 \mathrm{~Hz}, 1 \mathrm{H}, \mathrm{ArH}), 7.21(\mathrm{dd}, J=8.4 \mathrm{~Hz}, 2 \mathrm{H}, \mathrm{ArH}), 5.84(\mathrm{~s}, 1 \mathrm{H}, \mathrm{CH}), 3.73(\mathrm{~d}, J=11.2 \mathrm{~Hz}$, 2H, $\left.\mathrm{CH}_{2}\right), 3.64\left(\mathrm{~d}, J=11.2 \mathrm{~Hz}, 2 \mathrm{H}, \mathrm{CH}_{2}\right), 1.55\left(\mathrm{~s}, 3 \mathrm{H}, \mathrm{CH}_{3}\right), 1.32\left(\mathrm{~s}, 9 \mathrm{H}, \mathrm{CH}_{3}\right), 1.29$ (s, 9H, $\left.\mathrm{CH}_{3}\right), 0.78\left(\mathrm{~s}, 3 \mathrm{H}, \mathrm{CH}_{3}\right) ;{ }^{13} \mathrm{C}\left\{{ }^{1} \mathrm{H}\right\} \mathrm{NMR}\left(101 \mathrm{MHz}, \mathrm{CDCl}_{3}\right) \delta 150.9(\mathrm{C}), 149.7(\mathrm{C}), 138.4(\mathrm{C})$, $133.1(\mathrm{C}), 132.8(\mathrm{CH}), 130.6(\mathrm{C}), 130.0(\mathrm{C}), 130.4(\mathrm{CH}), 127.1(\mathrm{CH}), 126.3(\mathrm{CH}), 123.6(\mathrm{CH})$, $100.0(\mathrm{CH}), 77.7\left(\mathrm{CH}_{2}\right), 34.7(\mathrm{C}), 34.5(\mathrm{C}), 31.3\left(\mathrm{CH}_{3}\right), 31.2\left(\mathrm{CH}_{3}\right), 23.2\left(\mathrm{CH}_{3}\right), 21.8\left(\mathrm{CH}_{3}\right)$; MS (EI, positive mode): $\mathrm{m} / z 412\left([\mathrm{M}]^{+}\right)$; Anal. Calcd for $\mathrm{C}_{26} \mathrm{H}_{36} \mathrm{O}_{2} \mathrm{~S}: \mathrm{C}, 75.58 ; \mathrm{H}, 8.79 \%$; found: C, $75.20 ; \mathrm{H}, 9.14 \%$. 


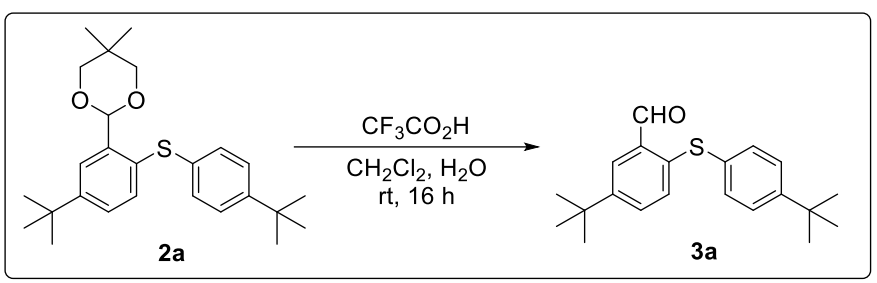

Synthesis of 5-tert-Butyl-2-(4-tert-butylphenylthio)benzaldehyde 3a. Compound 2a (0.900 $\mathrm{g}, 2.18 \mathrm{mmol})$ and trifluoroacetic acid $(5.0 \mathrm{~mL})$ was dissolved in a solution of THF $(10 \mathrm{~mL})$ and water $(10 \mathrm{~mL})$. After being stirred at room temperature for $2 \mathrm{~h}$, the solution was extracted with dichloromethane. After the organic layer was washed with water, the organic layers were dried over anhydrous sodium sulfate, filtered, and dried under reduced pressure to give crude yellow oil. Purification of the crude oil by column chromatography on silica gel (eluent: hexane/dichloromethane $\quad=\quad 1 / 1, \quad \mathrm{v} / \mathrm{v}) \quad$ afforded 5-tert-butyl-2-(4-tert-butylphenylthio)benzaldehyde 3a (0.604 g, $1.85 \mathrm{mmol}, 85 \%)$ as yellow oil. 3a: ${ }^{1} \mathrm{H}$ NMR (400 MHz, $\left.\mathrm{CDCl}_{3}\right) \delta 10.41(\mathrm{~s}, 1 \mathrm{H}, \mathrm{CH}), 7.90(\mathrm{~d}, J=2.4 \mathrm{~Hz}, 1 \mathrm{H}, \mathrm{ArH}), 7.47$ (dd, $J=2.4,8.4 \mathrm{~Hz}, 1 \mathrm{H}, \operatorname{ArH}), 7.39$ (d, $J=8.8 \mathrm{~Hz}, 2 \mathrm{H}, \mathrm{ArH}), 7.33$ (d, $J=8.8 \mathrm{~Hz}, 2 \mathrm{H}, \mathrm{ArH})$, $7.08(\mathrm{~d}, J=8.4 \mathrm{~Hz}, 1 \mathrm{H}, \mathrm{ArH}), 1.32\left(\mathrm{~s}, 9 \mathrm{H}, \mathrm{CH}_{3}\right), 1.32\left(\mathrm{~s}, 9 \mathrm{H}, \mathrm{CH}_{3}\right) ;{ }^{13} \mathrm{C}\left\{{ }^{1} \mathrm{H}\right\} \mathrm{NMR}(101 \mathrm{MHz}$, $\left.\mathrm{CDCl}_{3}\right) \delta 191.9(\mathrm{CH}), 151.4(\mathrm{C}), 149.9(\mathrm{C}), 138.4(\mathrm{C}), 133.7(\mathrm{C}), 132.5(\mathrm{CH}), 131.6(\mathrm{CH})$, $130.8(\mathrm{CH}), 130.3(\mathrm{C}), 128.1(\mathrm{CH}), 126.6(\mathrm{CH}), 34.64(\mathrm{C}), 34.58(\mathrm{C}), 31.2\left(\mathrm{CH}_{3}\right), 31.1\left(\mathrm{CH}_{3}\right)$; MS (EI, positive mode): $m / z 326\left([\mathrm{M}]^{+}\right)$; Anal. Calcd for $\mathrm{C}_{21} \mathrm{H}_{26} \mathrm{OS}$ : C, 77.25; $\mathrm{H}, 8.03 \%$; found: C, 76.95; H, 8.26\%.

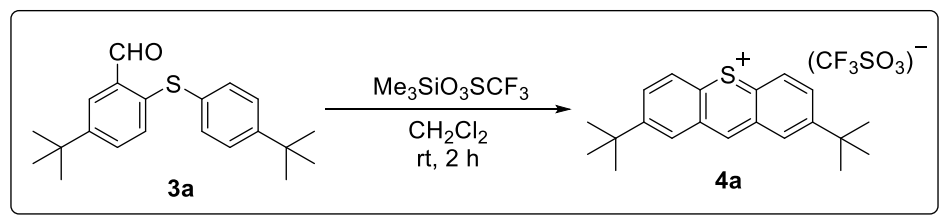

Reaction of 5-tert-Butyl-2-(4-tert-butylphenylthio)benzaldehyde 3a with Trimethylsilyl Trifluoromethanesulfonate (Me3SiOTf). $\mathrm{Me}_{3} \operatorname{SiOTf}(0.067 \mathrm{~g}, 0.30 \mathrm{mmol})$ was added to a solution of 5-tert-butyl-2-(4-tert-butylphenylthio)benzaldehyde $\mathbf{3 a}(0.050 \mathrm{~g}, 0.15 \mathrm{mmol})$ in dichloromethane $(6 \mathrm{~mL})$ at room temperature. After the solution was stirred at room 
temperature for $2 \mathrm{~h}$, all volatile substances were removed under reduced pressure. The resulting red oil was washed with cold dichloromethane, and dried under reduced pressure to give 2,7-di-tert-butylthioxanthylium trifluoromethanesulfonate 4a $(0.069 \mathrm{~g}, 0.15 \mathrm{mmol}$, quant.) as reddish orange oil. 4a: ${ }^{1} \mathrm{H}$ NMR $\left(400 \mathrm{MHz}, \mathrm{CD}_{3} \mathrm{CN}\right) \delta 10.18(\mathrm{~s}, 1 \mathrm{H}, \mathrm{ArH}), 8.77$ (d, $J=2.0 \mathrm{~Hz}, 2 \mathrm{H}, \mathrm{ArH}), 8.70(\mathrm{~d}, J=9.0 \mathrm{~Hz}, 2 \mathrm{H}, \mathrm{ArH}), 8.55(\mathrm{dd}, J=2.0,9.0 \mathrm{~Hz}, 2 \mathrm{H}, \mathrm{ArH}), 1.52$ $\left(\mathrm{s}, 9 \mathrm{H}, \mathrm{CH}_{3}\right) ;{ }^{13} \mathrm{C}\left\{{ }^{1} \mathrm{H}\right\} \mathrm{NMR}\left(101 \mathrm{MHz}, \mathrm{CD}_{3} \mathrm{CN}\right) \delta 161.1(\mathrm{CH}), 155.9(\mathrm{C}), 147.6(\mathrm{C}), 138.9$ $(\mathrm{CH}), 133.0(\mathrm{CH}), 131.6(\mathrm{C}), 128.2(\mathrm{CH}), 124.7$ (quart, $\left.J=281 \mathrm{~Hz}, \mathrm{CF}_{3}\right), 36.5(\mathrm{C}), 30.9$ $\left(\mathrm{CH}_{3}\right) ;{ }^{19} \mathrm{~F}$ NMR (376 MHz, CD $3 \mathrm{CN}$ ) $\delta-79.4$ (s); MS (ESI-TOF, positive mode): $\mathrm{m} / z, 309$ ([M-OTf $\left.]^{+}\right)$; HRMS (ESI-TOF, positive mode): $\mathrm{m} / \mathrm{z}$ found $309.1690\left([\mathrm{M}-\mathrm{OTf}]^{+}\right)$, calcd for $\mathrm{C}_{21} \mathrm{H}_{25} \mathrm{~S}: 309.1677$.

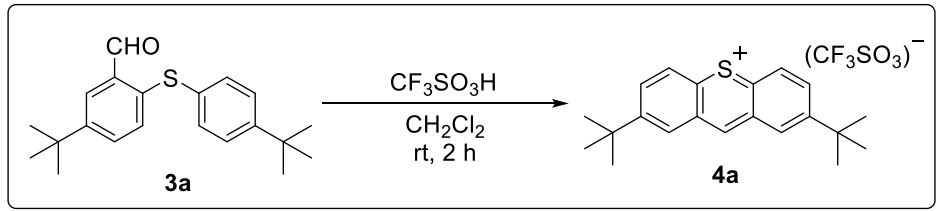

Reaction of 5-tert-Butyl-2-(4-tert-butylphenylthio)benzaldehyde $\quad 3 a \quad$ with Trifluoromethanesulfonic acid (TfOH). TfOH $(0.048 \mathrm{~g}, 0.32 \mathrm{mmol})$ was added to a solution of 5-tert-butyl-2-(4-tert-butylphenylthio)benzaldehyde $\quad 3 \mathbf{a} \quad(0.039 \mathrm{~g}, \quad 0.12 \mathrm{mmol})$ in dichloromethane $(6 \mathrm{~mL})$ at room temperature. After the solution was stirred at room temperature for $2 \mathrm{~h}$, all volatile substances were removed under reduced pressure. The resulting red oil was washed with cold dichloromethane, and dried under reduced pressure to give 2,7-di-tert-butylthioxanthylium trifluoromethanesulfonate $4 \mathbf{a}(0.055 \mathrm{~g}, 0.12 \mathrm{mmol}$, quant.) as reddish orange oil.

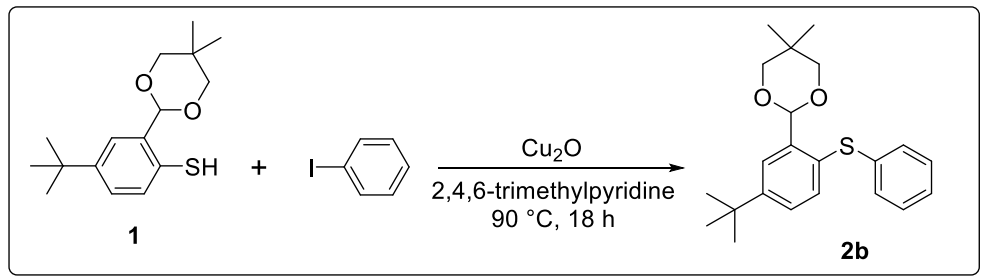

Synthesis of 2-[(5-tert-Butyl)-2-(phenylthiol)phenyl]-5,5-dimethyl-1,3-dioxane $2 \mathrm{~b}$. Benzenethiol 1 (791 mg, $2.82 \mathrm{mmol}$ ), iodobenzene (1.195 g, $5.86 \mathrm{mmol}$ ), and copper(I) oxide 
(805 mg, $5.63 \mathrm{mmol}$ ) were dissolved in 2,4,6-trimethylpyridine (7 mL). After being stirred at $90{ }^{\circ} \mathrm{C}$ for $18 \mathrm{~h}$ using an oil bath, the reaction mixture was allowed to cool to room temperature. After the solvent was removed under reduced pressure, purification of the crude product by column chromatography on silica gel (eluent: hexane/EtOAc $=10 / 1$, v/v) afforded 2-[(5-tert-butyl)-2-(phenylthiol)phenyl]-5,5-dimethyl-1,3-dioxane $2 \mathbf{b}(678 \mathrm{mg}, 1.90 \mathrm{mmol}$, $67 \%)$ as pale yellow oil. 2b: ${ }^{1} \mathrm{H}$ NMR $\left(400 \mathrm{MHz}, \mathrm{CDCl}_{3}\right) \delta 7.80(\mathrm{~d}, J=2.4 \mathrm{~Hz}, 1 \mathrm{H}, \mathrm{ArH})$, $7.30(\mathrm{dd}, J=2.4,8.4 \mathrm{~Hz}, 1 \mathrm{H}, \mathrm{ArH}), 7.26-7.24$ (m, 5H, ArH), 7.19-7.15 (m, 1H, ArH), 5.84 (s, 1H, CH), 3.73 (d, $\left.J=10.0 \mathrm{~Hz}, 2 \mathrm{H}, \mathrm{CH}_{2}\right), 3.62$ (d, $\left.J=10.0 \mathrm{~Hz}, 2 \mathrm{H}, \mathrm{CH}_{2}\right), 1.33\left(\mathrm{~s}, 9 \mathrm{H}, \mathrm{CH}_{3}\right)$, $1.32\left(\mathrm{~s}, 3 \mathrm{H}, \mathrm{CH}_{3}\right), 0.77\left(\mathrm{~s}, 3 \mathrm{H}, \mathrm{CH}_{3}\right) ;{ }^{13} \mathrm{C}\left\{{ }^{1} \mathrm{H}\right\} \mathrm{NMR}\left(101 \mathrm{MHz}, \mathrm{CDCl}_{3}\right) \delta 151.5(\mathrm{C}), 139.1$ (C), $137.3(\mathrm{C}), 133.4(\mathrm{CH}), 129.8(\mathrm{CH}), 129.7(\mathrm{C}), 128.9(\mathrm{CH}), 127.0(\mathrm{CH}), 126.2(\mathrm{CH})$, $123.6(\mathrm{CH}), 99.9(\mathrm{CH}), 77.8\left(\mathrm{CH}_{2}\right), 34.8(\mathrm{C}), 31.2\left(\mathrm{CH}_{3}\right), 30.2(\mathrm{C}), 23.2\left(\mathrm{CH}_{3}\right), 21.8\left(\mathrm{CH}_{3}\right)$; MS (EI, positive mode): $\mathrm{m} / \mathrm{z} 356\left([\mathrm{M}]^{+}\right)$; Anal. Calcd for $\mathrm{C}_{22} \mathrm{H}_{28} \mathrm{O}_{2} \mathrm{~S}: \mathrm{C}, 74.12 ; \mathrm{H}, 7.92 \%$; found: $\mathrm{C}, 73.91 ; \mathrm{H}, 8.05 \%$.

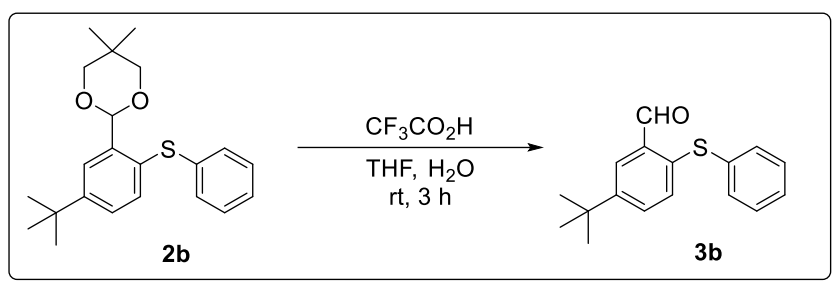

Synthesis of 5-tert-Butyl-2-(phenylthio)benzaldehyde 3b. Compound 2b (0.200 g, 0.561 mmol) and trifluoroacetic acid $(5.0 \mathrm{~mL})$ was dissolved in a solution of THF $(5 \mathrm{~mL})$ and water $(5 \mathrm{~mL})$. After being stirred at room temperature for $3 \mathrm{~h}$, the solution was extracted with dichloromethane. After the organic layer was washed with water, the organic layers were dried over anhydrous sodium sulfate, filtered, and dried under reduced pressure to give crude yellow oil. Purification of the crude oil by column chromatography on silica gel (eluent: hexane/dichloromethane $=1 / 1, \mathrm{v} / \mathrm{v})$ afforded 5-tert-butyl-2-(phenylthio)benzaldehyde $\mathbf{3 b}$ (0.085 g, $0.32 \mathrm{mmol}, 57 \%)$ as yellow oil. 3b: ${ }^{1} \mathrm{H}$ NMR $\left(400 \mathrm{MHz}, \mathrm{CDCl}_{3}\right) \delta 10.43(\mathrm{~s}, 1 \mathrm{H}$, CH), $7.92(\mathrm{~d}, J=2.4 \mathrm{~Hz}, 1 \mathrm{H}, \mathrm{ArH}), 7.48(\mathrm{dd}, J=2.4,8.3 \mathrm{~Hz}, 1 \mathrm{H}, \mathrm{ArH}), 7.38-7.30(\mathrm{~m}, 5 \mathrm{H}$, $\operatorname{ArH}), 7.13(\mathrm{~d}, J=8.3 \mathrm{~Hz}, 1 \mathrm{H}, \mathrm{ArH}), 1.33\left(\mathrm{~s}, 9 \mathrm{H}, \mathrm{CH}_{3}\right) ;{ }^{13} \mathrm{C}\left\{{ }^{1} \mathrm{H}\right\} \mathrm{NMR}\left(101 \mathrm{MHz}, \mathrm{CDCl}_{3}\right) \delta$ $192.0(\mathrm{CH}), 150.4(\mathrm{C}), 137.5(\mathrm{C}), 134.4(\mathrm{C}), 134.1(\mathrm{C}), 132.2(\mathrm{CH}), 131.7(\mathrm{CH}), 131.5(\mathrm{CH})$, 
$129.6(\mathrm{CH}), 128.0(\mathrm{CH}), 127.9(\mathrm{CH}), 34.7(\mathrm{C}), 31.1\left(\mathrm{CH}_{3}\right)$; MS (EI, positive mode): $\mathrm{m} / z 270$ $\left([\mathrm{M}]^{+}\right)$; Anal. Calcd for $\mathrm{C}_{17} \mathrm{H}_{18} \mathrm{OS}: \mathrm{C}, 75.52 ; \mathrm{H}, 6.71 \%$; found: $\mathrm{C}, 75.80 ; \mathrm{H}, 6.97 \%$.

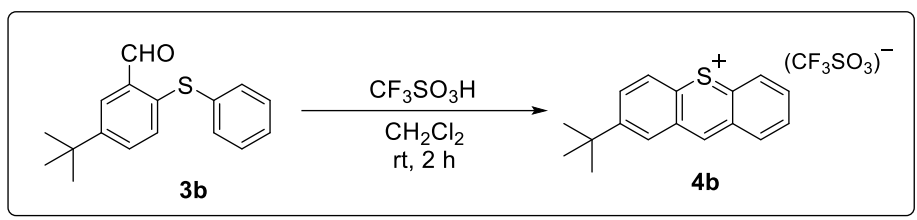

Reaction of 5-tert-Butyl-2-(phenylthio)benzaldehyde $3 b$ with TfOH. Trifluoromethanesulfonic acid $(0.030 \mathrm{~g}, 0.27 \mathrm{mmol})$ was added to a solution of 5-tert-butyl-2-(phenylthio)benzaldehyde $3 \mathbf{b}(0.030 \mathrm{~g}, 0.11 \mathrm{mmol})$ in dichloromethane $(7 \mathrm{~mL})$ at room temperature. After the solution was stirred at room temperature for $2 \mathrm{~h}$, all volatile substances were removed under reduced pressure. The resulting red oil was washed with cold dichloromethane, and dried under reduced pressure to give 2-tert-butylthioxanthylium trifluoromethanesulfonate $\mathbf{4 b}\left(0.044 \mathrm{~g}, 0.11 \mathrm{mmol}\right.$, quant.) as orange oil. 4b: ${ }^{1} \mathrm{H}$ NMR (400 $\left.\mathrm{MHz}, \mathrm{CD}_{3} \mathrm{CN}\right) \delta 10.22(\mathrm{~s}, 1 \mathrm{H}, \mathrm{CH}), 8.85(\mathrm{~d}, J=8.4 \mathrm{~Hz}, 1 \mathrm{H}, \mathrm{ArH}), 8.81(\mathrm{~d}, J=2.0 \mathrm{~Hz}, 1 \mathrm{H}$, ArH), 8.77-8.73 (m, 2H, ArH), 8.60 (dd, $J=2.0,8.8 \mathrm{~Hz}, 1 \mathrm{H}, \mathrm{ArH}), 8.41$ (t, $J=7.8 \mathrm{~Hz}, 1 \mathrm{H}$, $\mathrm{ArH}), 8.21(\mathrm{t}, J=7.6 \mathrm{~Hz}, 1 \mathrm{H}, \mathrm{ArH}), 1.53\left(\mathrm{~s}, 9 \mathrm{H}, \mathrm{CH}_{3}\right) ;{ }^{13} \mathrm{C}\left\{{ }^{1} \mathrm{H}\right\} \mathrm{NMR}\left(101 \mathrm{MHz}, \mathrm{CD}_{3} \mathrm{CN}\right) \delta$ $161.6(\mathrm{CH}), 156.0(\mathrm{C}), 149.4(\mathrm{C}), 148.2(\mathrm{C}), 139.4(\mathrm{CH}), 139.3(\mathrm{CH}), 138.1(\mathrm{CH}), 133.2(\mathrm{CH})$, $132.2(\mathrm{CH}), 131.5(\mathrm{C}), 131.3(\mathrm{C}), 128.4(\mathrm{CH}), 128.3(\mathrm{CH}), 121.0$ (quart, $J_{\mathrm{CF}}=317 \mathrm{~Hz}$, $\left.\mathrm{CF}_{3} \mathrm{SO}_{3}{ }^{-}\right), 36.5(\mathrm{C}), 30.9\left(\mathrm{CH}_{3}\right) ;{ }^{19} \mathrm{~F}$ NMR $\left(376 \mathrm{MHz}, \mathrm{CD}_{3} \mathrm{CN}\right) \delta-79.4$ (s); MS (ESI-TOF, positive mode): $m / z 253$ ([M-OTf] $]^{+}$); HRMS (ESI-TOF, positive mode): $m / z$ found 253.1060 $\left([\mathrm{M}-\mathrm{OTf}]^{+}\right)$, calcd for $\mathrm{C}_{17} \mathrm{H}_{17} \mathrm{~S}: 253.1051$.

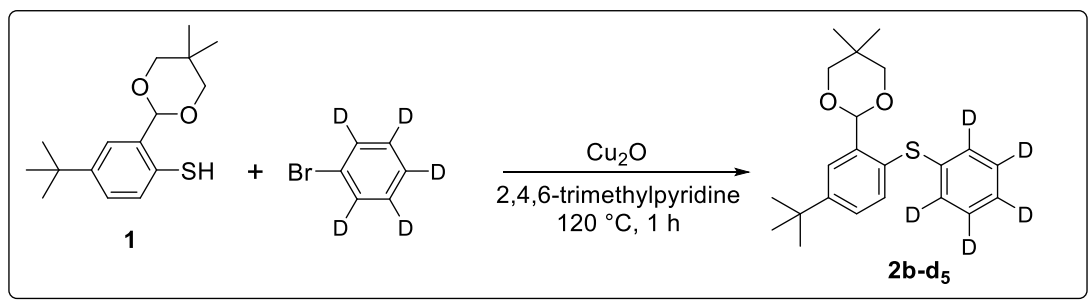

Synthesis of 2-[(5-tert-Butyl)-2-((phenyl-d5)thio)phenyl]-5,5-dimethyl-1,3-dioxane 2b-d5. Benzenethiol 1 (1.164 g, $4.15 \mathrm{mmol})$, bromobenzene- $d_{5}(0.810 \mathrm{~g}, 5.00 \mathrm{mmol})$, and copper(I) oxide (1.244 g, $8.55 \mathrm{mmol})$ were dissolved in 2,4,6-trimethylpyridine (8 mL). After being 
stirred at $120{ }^{\circ} \mathrm{C}$ for $1 \mathrm{~h}$ using an oil bath, the reaction mixture was allowed to cool to room temperature. After the solvent was removed under reduced pressure, purification of the crude product by column chromatography on silica gel (eluent: hexane/dichloromethane $=1 / 1, \mathrm{v} / \mathrm{v}$ ) afforded 2-[(5-tert-butyl)-2-((phenyl- $\left.d_{5}\right)$ thio)phenyl]-5,5-dimethyl-1,3-dioxane 2b-d5 (0.250 g, $0.691 \mathrm{mmol}, 17 \%)$ as pale yellow oil. 2b-d5: ${ }^{1} \mathrm{H} \mathrm{NMR}\left(400 \mathrm{MHz}, \mathrm{CDCl}_{3}\right) \delta 7.80(\mathrm{~d}, J=2.2$ $\mathrm{Hz}, 1 \mathrm{H}, \mathrm{ArH}), 7.30$ (dd, $J=2.2,8.3 \mathrm{~Hz}, 1 \mathrm{H}, \mathrm{ArH}), 7.25$ (d, J=8.3 Hz, 1H, ArH), 5.84 (s, 1H, $\mathrm{CH}), 3.73\left(\mathrm{~d}, J=10.8 \mathrm{~Hz}, 2 \mathrm{H}, \mathrm{CH}_{2}\right), 3.63\left(\mathrm{~d}, J=10.8 \mathrm{~Hz}, 2 \mathrm{H}, \mathrm{CH}_{2}\right), 1.33\left(\mathrm{~s}, 9 \mathrm{H}, \mathrm{CH}_{3}\right), 1.32$ (s, 3H, $\left.\mathrm{CH}_{3}\right), 0.77\left(\mathrm{~s}, 3 \mathrm{H}, \mathrm{CH}_{3}\right) ;{ }^{13} \mathrm{C}\left\{{ }^{1} \mathrm{H}\right\} \mathrm{NMR}\left(101 \mathrm{MHz}, \mathrm{CDCl}_{3}\right) \delta 151.3(\mathrm{C}), 139.0(\mathrm{C})$, $137.0(\mathrm{C}), 133.3(\mathrm{CH}), 129.7(\mathrm{C}), 129.3\left(\mathrm{t}, J_{\mathrm{CD}}=24.2 \mathrm{~Hz}, \mathrm{CD}\right), 128.4\left(\mathrm{t}, J_{\mathrm{CD}}=24.2 \mathrm{~Hz}, \mathrm{CD}\right)$, $126.9(\mathrm{CH}), 125.7\left(\mathrm{t}, J_{\mathrm{CD}}=24.2 \mathrm{~Hz}, \mathrm{CD}\right), 123.5(\mathrm{CH}), 99.8(\mathrm{CH}), 77.7\left(\mathrm{CH}_{2}\right), 34.7(\mathrm{C}), 31.2$ $\left(\mathrm{CH}_{3}\right), 30.2(\mathrm{C}), 23.2\left(\mathrm{CH}_{3}\right), 21.8\left(\mathrm{CH}_{3}\right)$; MS (EI, positive mode): $\mathrm{m} / z 361\left([\mathrm{M}]^{+}\right)$; HRMS (EI, positive mode): $m / z$ found $361.2116\left([\mathrm{M}]^{+}\right)$, calcd for $\mathrm{C}_{22} \mathrm{H}_{23} \mathrm{D}_{5} \mathrm{O}_{2} \mathrm{~S}: 361.2124$.

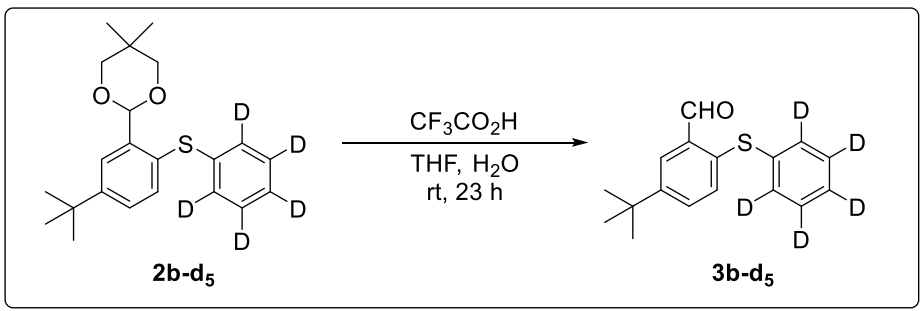

Synthesis of 5-tert-Butyl-2-[(phenyl-d5)thio]benzaldehyde 3b-d5. Compound 2b-d5 (0.100 $\mathrm{g}, 0.28 \mathrm{mmol})$ and trifluoroacetic acid $(4.0 \mathrm{~mL})$ was dissolved in a solution of THF $(5 \mathrm{~mL})$ and water $(5 \mathrm{~mL})$. After being stirred at room temperature for $23 \mathrm{~h}$, the solution was extracted with dichloromethane. After the organic layer was washed with water, the organic layers were dried over anhydrous sodium sulfate, filtered, and dried under reduced pressure to give crude yellow oil. Purification of the crude oil by column chromatography on silica gel (eluent: hexane/dichloromethane $=1 / 1, \mathrm{v} / \mathrm{v})$ afforded 5-tert-butyl-2-[(phenyl- $\left.d_{5}\right)$ thio $]$ benzaldehyde 3b-d5 (0.033 g, 0.12 mmol, 43\%) as yellow oil. 3b-d5: ${ }^{1} \mathrm{H}$ NMR (400 MHz, $\left.\mathrm{CDCl}_{3}\right) \delta 10.43$ (s, 1H, CH), $7.92(\mathrm{~d}, J=2.3 \mathrm{~Hz}, 1 \mathrm{H}, \mathrm{ArH}), 7.48(\mathrm{dd}, J=2.3,8.3 \mathrm{~Hz}, 1 \mathrm{H}, \mathrm{ArH}), 7.12(\mathrm{~d}, J=8.3$ $\mathrm{Hz}, 1 \mathrm{H}, \mathrm{ArH}), 1.33\left(\mathrm{~s}, 9 \mathrm{H}, \mathrm{CH}_{3}\right) ;{ }^{13} \mathrm{C}\left\{{ }^{1} \mathrm{H}\right\} \mathrm{NMR}\left(101 \mathrm{MHz}, \mathrm{CDCl}_{3}\right) \delta 192.0(\mathrm{CH}), 150.4(\mathrm{C})$, $137.5(\mathrm{C}), 134.1(\mathrm{C}), 134.0(\mathrm{C}), 131.8\left(\mathrm{t}, J_{\mathrm{CD}}=25.3 \mathrm{~Hz}, \mathrm{CD}\right), 131.7(\mathrm{CH}), 131.5(\mathrm{CH}), 129.1$ $\left(\mathrm{t}, J_{\mathrm{CD}}=24.2 \mathrm{~Hz}, \mathrm{CD}\right), 128.0(\mathrm{CH}), 127.4\left(\mathrm{t}, J_{\mathrm{CD}}=25.3 \mathrm{~Hz}, \mathrm{CD}\right), 34.7(\mathrm{C}), 31.1\left(\mathrm{CH}_{3}\right) ; \mathrm{MS}$ 
(EI, positive mode) $m / z 275\left([\mathrm{M}]^{+}\right)$; HRMS (EI, positive mode): $m / z$ found $275.1406\left([\mathrm{M}]^{+}\right.$), calcd for $\mathrm{C}_{17} \mathrm{H}_{13} \mathrm{D}_{5} \mathrm{OS}: 275.1392$.

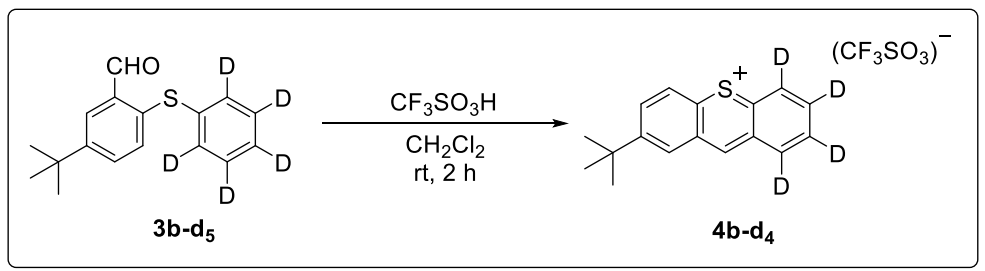

Reaction of 5-(tert-Butyl)-2-[(phenyl-d5)thio]benzaldehyde $3 \mathrm{~b}-\mathrm{d} 5$ with TfOH. Trifluoromethanesulfonic acid $(0.029 \mathrm{~g}, 0.28 \mathrm{mmol})$ was added to a solution of $\mathbf{3 b - d} \mathbf{5}(0.030 \mathrm{~g}$, $0.11 \mathrm{mmol})$ in dichloromethane $(5 \mathrm{~mL})$ at room temperature. After the solution was stirred at room temperature for $2 \mathrm{~h}$, all volatile substances were removed under reduced pressure. The resulting red oil was washed with cold dichloromethane, and dried under reduced pressure to give 2-tert-butylthioxanthylium-5,6,7,8- $d_{4}$ trifluoromethanesulfonate $\mathbf{4 b - d} \mathbf{4}(0.041 \mathrm{~g}, 0.10$ mmol, 91\%) as orange oil. 4b-d4: ${ }^{1} \mathrm{H}$ NMR (400 MHz, $\left.\mathrm{CD}_{3} \mathrm{CN}\right) \delta 10.23$ (s, 1H, ArH), 8.81 (d, $J=2.4 \mathrm{~Hz}, 1 \mathrm{H}, \mathrm{ArH}), 8.74(\mathrm{~d}, J=8.8 \mathrm{~Hz}, 1 \mathrm{H}, \mathrm{ArH}), 8.60(\mathrm{dd}, J=2.4,8.8 \mathrm{~Hz}, 1 \mathrm{H}, \mathrm{ArH}), 1.53$ (s, 3H, $\left.\mathrm{CH}_{3}\right) ;{ }^{13} \mathrm{C}\left\{{ }^{1} \mathrm{H}\right\}$ NMR (101 MHz, CD $\left.{ }_{3} \mathrm{CN}\right) \delta 161.6(\mathrm{CH}), 156.1(\mathrm{C}), 149.3(\mathrm{C}), 148.2$ (C), $139.5(\mathrm{CH}), 133.2(\mathrm{CH}), 131.5(\mathrm{C}), 131.3(\mathrm{C}), 128.3(\mathrm{CH}), 127.5(\mathrm{C}), 127.3(\mathrm{C}), 126.3$ (C), 124.8 (C), 121.5 (quart, $J=320 \mathrm{~Hz}, \mathrm{CF}_{3}$ ), $36.5(\mathrm{C}), 30.9\left(\mathrm{CH}_{3}\right) ;{ }^{19} \mathrm{~F}$ NMR (376 MHz, $\mathrm{CD}_{3} \mathrm{CN}$ ) $\delta-79.4$ (s); MS (ESI-TOF, positive mode): $m / z 257$ ([M-OTf] ${ }^{+}$); HRMS (ESI-TOF, positive mode): $\mathrm{m} / \mathrm{z}$ found $257.1318\left([\mathrm{M}-\mathrm{OTf}]^{+}\right)$, calcd for $\mathrm{C}_{17} \mathrm{H}_{13} \mathrm{D}_{4} \mathrm{~S}: 257.1302$.

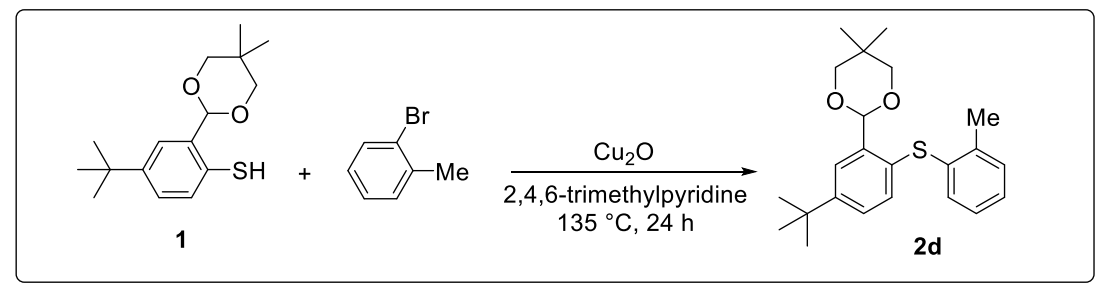

Synthesis of 2-[(5-tert-Butyl)-2-(2-methylphenylthio)phenyl]-5,5-dimethyl-1,3-dioxane 2d. Benzenethiol 1 (2.601 g, $9.28 \mathrm{mmol})$, 2-bromo-1-methylbenzene (2.036 g, $11.1 \mathrm{mmol})$, and copper(I) oxide (2.658 g, $18.6 \mathrm{mmol})$ were dissolved in 2,4,6-trimethylpyridine (30 mL). After being stirred at $135{ }^{\circ} \mathrm{C}$ for $24 \mathrm{~h}$ using an oil bath, the reaction mixture was allowed to 
cool to room temperature. After the solvent was removed under reduced pressure, purification of the crude product by column chromatography on silica gel (eluent: hexane/dichloromethane $\quad=\quad 4 / 1, \quad \mathrm{v} / \mathrm{v}) \quad$ afforded 2-[(5-tert-butyl)-2-(2-methylphenylthio)phenyl]-5,5-dimethyl-1,3-dioxane 2d (2.270 g, 6.12 mmol, 66\%) as colorless crystals. 2d: mp 71.3-72.5 ${ }^{\circ} \mathrm{C} ;{ }^{1} \mathrm{H}$ NMR $\left(400 \mathrm{MHz}, \mathrm{CDCl}_{3}\right) \delta 7.78$ $(\mathrm{d}, J=2.0 \mathrm{~Hz}, 1 \mathrm{H}, \mathrm{ArH}), 7.25(\mathrm{dd}, J=2.0,8.2 \mathrm{~Hz}, 1 \mathrm{H}, \mathrm{ArH}), 7.20(\mathrm{~d}, J=2.4 \mathrm{~Hz}, 1 \mathrm{H}, \mathrm{ArH})$, 7.18-7.06 (m, 3H, ArH), $7.02(\mathrm{~d}, J=8.4 \mathrm{~Hz}, 1 \mathrm{H}, \mathrm{ArH}), 5.80(\mathrm{~s}, 1 \mathrm{H}, \mathrm{CH}), 3.75(\mathrm{~d}, J=11.2 \mathrm{~Hz}$, 2H, $\left.\mathrm{CH}_{2}\right), 3.65$ (d, J=11.2 Hz, 2H, $\left.\mathrm{CH}_{2}\right), 2.38\left(\mathrm{~s}, 3 \mathrm{H}, \mathrm{CH}_{3}\right), 1.54\left(\mathrm{~s}, 3 \mathrm{H}, \mathrm{CH}_{3}\right), 1.32$ (s, 9H, $\left.\mathrm{CH}_{3}\right), 0.78\left(\mathrm{~s}, 3 \mathrm{H}, \mathrm{CH}_{3}\right) ;{ }^{13} \mathrm{C}\left\{{ }^{1} \mathrm{H}\right\} \mathrm{NMR}\left(101 \mathrm{MHz}, \mathrm{CDCl}_{3}\right) \delta 150.6(\mathrm{C}), 138.5(\mathrm{C}), 138.1(\mathrm{C})$, $135.3(\mathrm{C}), 131.67(\mathrm{CH}), 131.65(\mathrm{CH}), 130.3(\mathrm{CH}), 130.2(\mathrm{CH}), 126.92(\mathrm{CH}), 126.89(\mathrm{CH})$, $126.6(\mathrm{CH}), 123.4(\mathrm{CH}), 99.7(\mathrm{CH}), 77.7\left(\mathrm{CH}_{2}\right), 34.7(\mathrm{C}), 31.2\left(\mathrm{CH}_{3}\right), 30.2(\mathrm{C}), 23.2\left(\mathrm{CH}_{3}\right)$, $21.8\left(\mathrm{CH}_{3}\right), 20.5\left(\mathrm{CH}_{3}\right)$; MS (EI, positive mode): $\mathrm{m} / z 370\left([\mathrm{M}]^{+}\right)$; Anal. Calcd for $\mathrm{C}_{23} \mathrm{H}_{30} \mathrm{O}_{2} \mathrm{~S}$ : C, 74.55; H, 8.16\%; found: C, 74.24; H, 8.10\%.

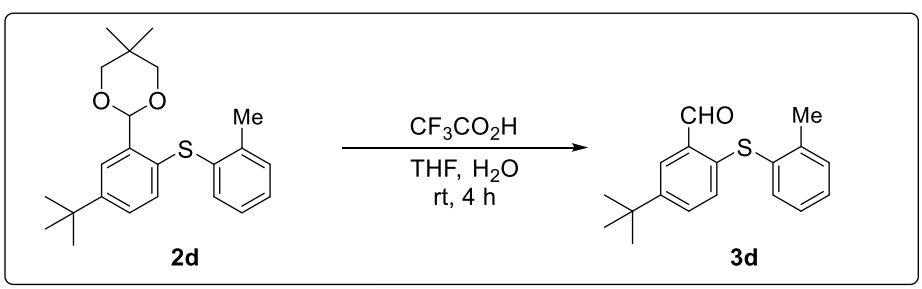

Synthesis of 5-tert-Butyl-2-(2-methylphenylthio)benzaldehyde 3d. Compound 2d (0.923 g, $2.49 \mathrm{mmol})$ and trifluoroacetic acid $(5.0 \mathrm{~mL})$ was dissolved in a solution of THF $(5 \mathrm{~mL})$ and water $(5 \mathrm{~mL})$. After being stirred at room temperature for $4 \mathrm{~h}$, the solution was extracted with dichloromethane. After the organic layer was washed with water, the organic layers were dried over anhydrous sodium sulfate, filtered, and dried under reduced pressure to give crude yellow oil. Purification of the crude oil by column chromatography on silica gel (eluent: hexane/dichloromethane $\quad=\quad 1 / 1, \quad \mathrm{v} / \mathrm{v}) \quad$ afforded 5-tert-butyl-2-(2-methylphenylthio)benzaldehyde 3d (0.652 g, $2.29 \mathrm{mmol}, 92 \%)$ as pale yellow oil. 3d: ${ }^{1} \mathrm{H}$ NMR (400 MHz, $\left.\mathrm{CDCl}_{3}\right) \delta 10.38(\mathrm{~s}, 1 \mathrm{H}, \mathrm{CHO}), 7.90$ (d, J = $2.4 \mathrm{~Hz}, 1 \mathrm{H}$, ArH), $7.43(\mathrm{dd}, J=2.4,8.4 \mathrm{~Hz}, 1 \mathrm{H}, \operatorname{ArH}), 7.32$ (d, $J=7.8 \mathrm{~Hz}, 1 \mathrm{H}, \operatorname{ArH}), 7.29-7.27$ (m, 2H, 
ArH), 7.21-7.18 (m, 1H, ArH), 6.87 (d, $J=8.4 \mathrm{~Hz}, 1 \mathrm{H}, \mathrm{ArH}), 2.39$ (s, 3H, $\left.\mathrm{CH}_{3}\right), 1.32$ (s, 9H, $\left.\mathrm{CH}_{3}\right) ;{ }^{13} \mathrm{C}\left\{{ }^{1} \mathrm{H}\right\} \mathrm{NMR}\left(101 \mathrm{MHz}, \mathrm{CDCl}_{3}\right) \delta 191.8$ (CHO), $149.5(\mathrm{C}), 140.9$ (C), 137.9 (C), $134.1(\mathrm{CH}), 133.4(\mathrm{C}), 132.5(\mathrm{C}), 131.6(\mathrm{CH}), 130.8(\mathrm{CH}), 129.6(\mathrm{CH}), 128.73(\mathrm{CH}), 128.65$ (CH), $127.1(\mathrm{CH}), 34.5(\mathrm{C}), 31.1\left(\mathrm{CH}_{3}\right), 20.5\left(\mathrm{CH}_{3}\right)$; MS (EI, positive mode): $m / z 284\left([\mathrm{M}]^{+}\right)$; HRMS (EI, positive mode): $\mathrm{m} / z$ found $284.1222\left([\mathrm{M}]^{+}\right)$, calcd for $\mathrm{C}_{18} \mathrm{H}_{20} \mathrm{OS}: 284.1235$.

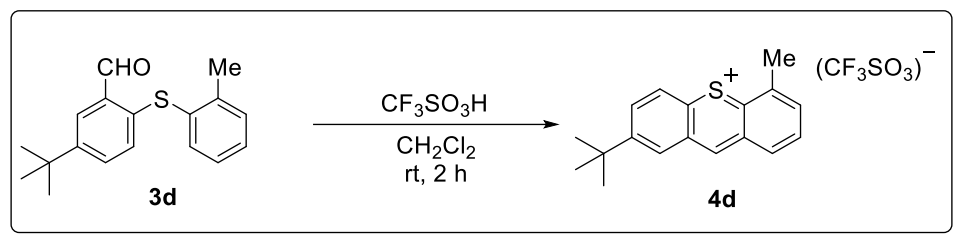

\section{Reaction of 5-tert-Butyl-2-(2-methylphenylthio)benzaldehyde 3d with TfOH.}

Trifluoromethanesulfonic acid $(0.452 \mathrm{~g}, 4.36 \mathrm{mmol})$ was added to a solution of compound $\mathbf{3 d}$ $(0.620 \mathrm{~g}, 2.18 \mathrm{mmol})$ in dichloromethane $(10 \mathrm{~mL})$ at room temperature. After the solution was stirred at room temperature for $2 \mathrm{~h}$, all volatile substances were removed under reduced pressure. The resulting red oil was washed with cold dichloromethane, and dried under reduced pressure to give 2-tert-butyl-5-methylthioxanthylium trifluoromethanesulfonate $\mathbf{4 d}$ (0.891 g, 2.14 mmol, 98\%) as waxy red solids. 4d: ${ }^{1} \mathrm{H}$ NMR (400 MHz, CD $\left.{ }_{3} \mathrm{CN}\right) \delta 10.20$ (s, 1H, ArH), $8.81(\mathrm{~d}, J=2.0 \mathrm{~Hz}, 1 \mathrm{H}, \operatorname{ArH}), 8.76(\mathrm{~d}, J=8.8 \mathrm{~Hz}, 1 \mathrm{H}, \operatorname{ArH}), 8.72(\mathrm{~d}, J=8.4 \mathrm{~Hz}$, 1H, ArH), 8.60 (dd, $J=2.0,8.8 \mathrm{~Hz}, 1 \mathrm{H}, \mathrm{ArH}), 8.27$ (d, $J=7.2 \mathrm{~Hz}, 1 \mathrm{H}, \mathrm{ArH}), 8.12$ (dd, $J=7.2$, $8.4 \mathrm{~Hz}, 1 \mathrm{H}, \mathrm{ArH}), 2.96\left(\mathrm{~s}, 3 \mathrm{H}, \mathrm{CH}_{3}\right), 1.81\left(\mathrm{~s}, 9 \mathrm{H}, \mathrm{CH}_{3}\right) ;{ }^{13} \mathrm{C}\left\{{ }^{1} \mathrm{H}\right\} \mathrm{NMR}\left(101 \mathrm{MHz}, \mathrm{CD}_{3} \mathrm{CN}\right) \delta$ $162.0(\mathrm{CH}), 156.1(\mathrm{C}), 148.9(\mathrm{C}), 147.3(\mathrm{C}), 140.1(\mathrm{CH}), 139.3(\mathrm{CH}), 137.3(\mathrm{C}), 136.7(\mathrm{CH})$, $133.0(\mathrm{CH}), 131.6(\mathrm{CH}), 131.1(\mathrm{C}), 128.5(\mathrm{CH}), 128.09(\mathrm{CH}), 121.4$ (quart, $J_{\mathrm{CF}}=317 \mathrm{~Hz}$, $\left.\mathrm{CF}_{3}\right), 36.5(\mathrm{C}), 30.9\left(\mathrm{CH}_{3}\right), 21.2\left(\mathrm{CH}_{3}\right) ;{ }^{19} \mathrm{~F} \mathrm{NMR}\left(376 \mathrm{MHz}, \mathrm{CD}_{3} \mathrm{CN}\right) \delta-79.4(\mathrm{~s}) ; \mathrm{MS}$ (ESI-TOF, positive mode): $m / z 267$ ([M-OTf $]^{+}$); Anal. Calcd for $\mathrm{C}_{19} \mathrm{H}_{19} \mathrm{~F}_{3} \mathrm{O}_{3} \mathrm{~S}_{2}: \mathrm{C}, 54.80 ; \mathrm{H}$, 4.60\%; found: C, 54.45; H, $4.35 \%$.

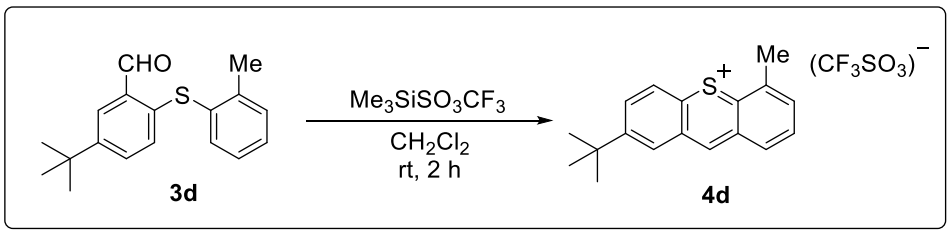


Reaction of 3d with Me3SiOTf. Trimethylsilyl trifluoromethanesulfonate $(0.160 \mathrm{~g}, 0.718$ mmol) was added to a solution of compound $\mathbf{3 d}(0.102 \mathrm{~g}, 0.359 \mathrm{mmol})$ in dichloromethane (5 $\mathrm{mL}$ ) at room temperature. After the solution was stirred at room temperature for $2 \mathrm{~h}$, the reaction mixture was added dropwise to cold $\mathrm{Et}_{2} \mathrm{O}(20 \mathrm{~mL})$. Purification of the resulting suspension using a centrifugal separator afforded 2-tert-butyl-5-methylthioxanthylium trifluoromethanesulfonate $\mathbf{4 f}(0.142 \mathrm{~g}, 0.341 \mathrm{mmol}, 95 \%)$ as red solids.

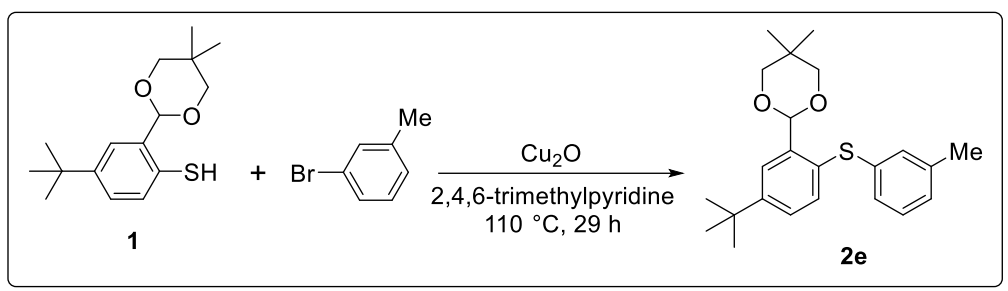

Synthesis of 2-[(5-tert-Butyl)-2-(3-methylphenylthio)phenyl]-5,5-dimethyl-1,3-dioxane 2e. Benzenethiol 1 (1.136 g, $4.05 \mathrm{mmol})$, 3-bromotoluene (0.840 g, $4.91 \mathrm{mmol})$, and copper(I) oxide (1.164 g, $8.13 \mathrm{mmol}$ ) were dissolved in 2,4,6-trimethylpyridine (15 mL). After being stirred at $110{ }^{\circ} \mathrm{C}$ for $29 \mathrm{~h}$ using an oil bath, the reaction mixture was allowed to cool to room temperature. After the solvent was removed under reduced pressure, purification of the crude product by column chromatography on silica gel (eluent: hexane/dichloromethane $=1 / 1$, v/v) afforded 2-[(5-tert-butyl)-2-(3-methylphenylthio)phenyl]-5,5-dimethyl-1,3-dioxane 2e (0.309 g, $0.834 \mathrm{mmol}, 21 \%)$ as colorless oil. 2e: ${ }^{1} \mathrm{H}$ NMR (400 MHz, $\left.\mathrm{CDCl}_{3}\right) \delta 7.79(\mathrm{~d}, J=2.0 \mathrm{~Hz}$, $1 \mathrm{H}, \mathrm{ArH}), 7.29(\mathrm{dd}, J=2.0,8.2 \mathrm{~Hz}, 1 \mathrm{H}, \mathrm{ArH}), 7.22(\mathrm{~d}, J=8.2 \mathrm{~Hz}, 1 \mathrm{H}, \operatorname{ArH}), 7.14(\mathrm{t}, J=7.6$ Hz, 1H, ArH), 7.12 (s, 1H, ArH), 7.04 (d, J=7.6 Hz, 1H, ArH), 6.99 (d, J=7.6 Hz, 1H, ArH), $5.84(\mathrm{~s}, 1 \mathrm{H}, \mathrm{CH}), 3.74$ (d, J = 10.4 Hz, 2H, $\left.\mathrm{CH}_{2}\right), 3.65$ (d, $\left.J=10.4 \mathrm{~Hz}, 2 \mathrm{H}, \mathrm{CH}_{2}\right), 2.29$ (s, 3H, $\left.\mathrm{CH}_{3}\right), 1.33$ (s, 9H, $\left.\mathrm{CH}_{3}\right), 1.32$ (s, 3H, $\left.\mathrm{CH}_{3}\right), 0.78$ (s, 3H, CH$) ;{ }^{13} \mathrm{C}\left\{{ }^{1} \mathrm{H}\right\}$ NMR (101 MHz, $\left.\mathrm{CDCl}_{3}\right) \delta 151.2(\mathrm{C}), 138.8(\mathrm{C} \times 2), 136.7(\mathrm{C}), 133.0(\mathrm{CH}), 130.8(\mathrm{CH}), 130.2(\mathrm{C}), 128.9(\mathrm{CH})$, $127.3(\mathrm{CH}), 127.3(\mathrm{CH}), 126.9(\mathrm{CH}), 123.5(\mathrm{CH}), 99.9(\mathrm{CH}), 77.8\left(\mathrm{CH}_{2}\right), 34.8(\mathrm{C}), 31.2$ $\left(\mathrm{CH}_{3}\right), 30.3(\mathrm{C}), 23.2\left(\mathrm{CH}_{3}\right), 21.9\left(\mathrm{CH}_{3}\right), 21.3\left(\mathrm{CH}_{3}\right)$; MS (EI, positive mode) $\mathrm{m} / z .370\left([\mathrm{M}]^{+}\right)$; Anal. Calcd for $\mathrm{C}_{23} \mathrm{H}_{30} \mathrm{O}_{2} \mathrm{~S}$ : C, 74.55; H, 8.16\%; found: C, 74.43; H, 7.90\%. 


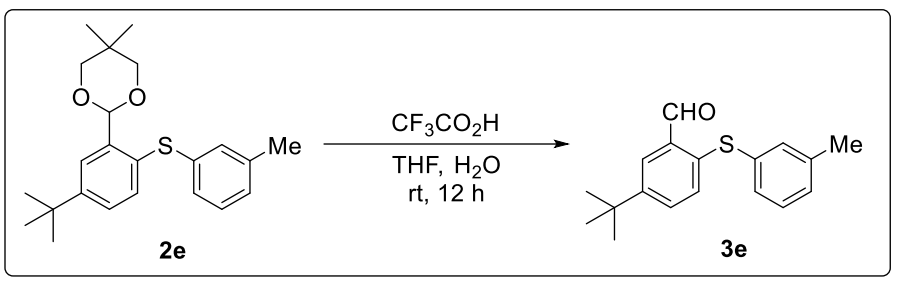

Synthesis of 5-tert-Butyl-2-(3-methylphenylthio)benzaldehyde 3e. Compound 2e (0.100 g, $0.270 \mathrm{mmol})$ and trifluoroacetic acid $(5.0 \mathrm{~mL})$ was dissolved in a solution of THF $(5 \mathrm{~mL})$ and water $(5 \mathrm{~mL})$. After being stirred at room temperature for $12 \mathrm{~h}$, the solution was extracted with dichloromethane. After the organic layer was washed with water, the organic layers were dried over anhydrous sodium sulfate, filtered, and dried under reduced pressure to give crude yellow oil. Purification of the crude oil by column chromatography on silica gel (eluent: hexane/dichloromethane $\quad=\quad 1 / 1, \quad \mathrm{v} / \mathrm{v}) \quad$ afforded 5-tert-butyl-2-(3-methylphenylthio)benzaldehyde $\mathbf{3 e}(0.074 \mathrm{~g}, 0.26 \mathrm{mmol}, 96 \%)$ as pale yellow oil. 3e: ${ }^{1} \mathrm{H}$ NMR (400 MHz, $\left.\mathrm{CDCl}_{3}\right) \delta 10.43$ (s, 1H, CHO), 7.91 (d, $J=2.4 \mathrm{~Hz}, 1 \mathrm{H}$, ArH), 7.47 (dd, $J=2.4,8.4 \mathrm{~Hz}, 1 \mathrm{H}, \mathrm{ArH}), 7.25-7.21$ (m, 2H, ArH), 7.16 (d, $J=8.0 \mathrm{~Hz}, 1 \mathrm{H}$, $\operatorname{ArH}), 7.12(\mathrm{~d}, J=8.4 \mathrm{~Hz}, 2 \mathrm{H}, \mathrm{ArH}), 2.33\left(\mathrm{~s}, 3 \mathrm{H}, \mathrm{CH}_{3}\right), 1.33\left(\mathrm{~s}, 9 \mathrm{H}, \mathrm{CH}_{3}\right) ;{ }^{13} \mathrm{C}\left\{{ }^{1} \mathrm{H}\right\} \mathrm{NMR}$ (101 MHz, $\left.\mathrm{CDCl}_{3}\right) \delta 191.9(\mathrm{CH}), 150.2$ (C), 139.4 (C), 137.8 (C), 133.9 (C), 133.9 (C), 132.8 $(\mathrm{CH}), 131.6(\mathrm{CH}), 131.3(\mathrm{CH}), 129.4(\mathrm{CH}), 128.8(\mathrm{CH}), 127.9(\mathrm{CH}), 34.6(\mathrm{C}), 31.1\left(\mathrm{CH}_{3}\right)$, $21.3\left(\mathrm{CH}_{3}\right)$; MS (EI, positive mode): $\mathrm{m} / z 284\left([\mathrm{M}]^{+}\right)$; Anal. Calcd for $\mathrm{C}_{18} \mathrm{H}_{20} \mathrm{OS}: \mathrm{C}, 76.01 ; \mathrm{H}$, 7.09\%; found: C, 75.95; H, 7.32\%.

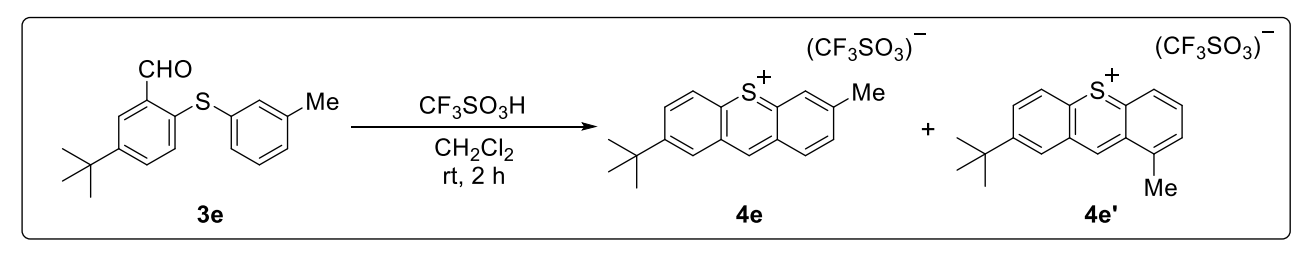

Reaction of 5-tert-Butyl-2-(4-methylphenylthio)benzaldehyde $3 \mathrm{e}$ with TfOH. Trifluoromethanesulfonic acid $(0.078 \mathrm{~g}, 0.52 \mathrm{mmol})$ was added to a solution of compound $\mathbf{3 e}$ $(0.100 \mathrm{~g}, 0.352 \mathrm{mmol})$ in dichloromethane $(4 \mathrm{~mL})$ at room temperature. After the solution was stirred at room temperature for $2 \mathrm{~h}$, all volatile substances were removed under reduced pressure. The formation of 2-tert-butyl-6-methylthioxanthylium trifluoromethanesulfonate $4 \mathbf{e}$ 
and 2-tert-butyl-8-methylthioxanthylium trifluoromethanesulfonate $4 \mathbf{e}^{\prime}$ was detected by NMR spectroscopy $\left(4 \mathbf{e}: 4 \mathbf{e}^{\prime}=88: 12\right)$. The resulting red oil was washed with cold $\mathrm{Et}_{2} \mathrm{O}$, and dried under reduced pressure to give $4 \mathbf{e}$ and $4 \mathbf{e}^{\prime}(0.126 \mathrm{~g}, 0.303 \mathrm{mmol}, 86 \%)$ as reddish orange oil. 4e and 4e': MS (ESI-TOF, positive mode): $m / z 267\left([\mathrm{M}-\mathrm{OTf}]^{+}\right)$; Anal. Calcd for $\mathrm{C}_{19} \mathrm{H}_{19} \mathrm{~F}_{3} \mathrm{O}_{3} \mathrm{~S}_{2}: \mathrm{C}, 54.80 ; \mathrm{H}, 4.60 \%$; found: $\mathrm{C}, 54.48 ; \mathrm{H}, 4.87 \%$. 4e: ${ }^{1} \mathrm{H}$ NMR $(400 \mathrm{MHz}$, $\left.\mathrm{CD}_{3} \mathrm{CN}\right) \delta 10.08(\mathrm{~s}, 1 \mathrm{H}, \mathrm{ArH}), 8.75(\mathrm{~d}, J=2.4 \mathrm{~Hz}, 1 \mathrm{H}, \mathrm{ArH}), 8.71(\mathrm{~d}, J=8.8 \mathrm{~Hz}, 1 \mathrm{H}, \mathrm{ArH})$, $8.66(\mathrm{~d}, J=8.8 \mathrm{~Hz}, 1 \mathrm{H}, \mathrm{ArH}), 8.55(\mathrm{~s}, 1 \mathrm{H}, \mathrm{ArH}), 8.54(\mathrm{dd}, J=2.0,8.8 \mathrm{~Hz}, 1 \mathrm{H}, \mathrm{ArH}), 8.04(\mathrm{dd}$, $J=2.0,8.8 \mathrm{~Hz}, 1 \mathrm{H}, \mathrm{ArH}), 2.80\left(\mathrm{~s}, 3 \mathrm{H}, \mathrm{CH}_{3}\right), 1.52\left(\mathrm{~s}, 9 \mathrm{H}, \mathrm{CH}_{3}\right) ;{ }^{13} \mathrm{C}\left\{{ }^{1} \mathrm{H}\right\} \mathrm{NMR}(101 \mathrm{MHz}$, $\left.\mathrm{CD}_{3} \mathrm{CN}\right) \delta 161.0(\mathrm{CH}), 150.9(\mathrm{C}), 144.9(\mathrm{C}), 138.3(\mathrm{C}), 135.2(\mathrm{C}), 131.6(\mathrm{CH}), 130.3(\mathrm{CH})$, $129.6(\mathrm{C}), 128.9(\mathrm{CH}), 127.8(\mathrm{C}), 127.1(\mathrm{CH}), 126.9(\mathrm{CH}), 126.1(\mathrm{C}), 121.9$ (quart, $J=320$ $\left.\mathrm{Hz}, \mathrm{CF}_{3}\right), 35.6(\mathrm{C}), 31.4\left(\mathrm{CH}_{3}\right), 21.7\left(\mathrm{CH}_{3}\right) .4 \mathbf{e}^{\prime}:{ }^{1} \mathrm{H}$ NMR $\left(400 \mathrm{MHz}, \mathrm{CD}_{3} \mathrm{CN}\right) \delta 10.38(\mathrm{~s}, 1 \mathrm{H}$, ArH), $8.92(\mathrm{~d}, J=2.4 \mathrm{~Hz}, 1 \mathrm{H}, \operatorname{ArH}), 8.70(\mathrm{~d}, J=9.2 \mathrm{~Hz}, 1 \mathrm{H}, \operatorname{ArH}), 8.59-8.56(\mathrm{~m}, 2 \mathrm{H}, \operatorname{ArH})$, 8.29-8.25 (m, 1H, ArH), 8.04-8.02 (m, 1H, ArH), 3.10 (s, 3H, CH $\mathrm{CH}_{3}, 1.53\left(\mathrm{~s}, 9 \mathrm{H}, \mathrm{CH}_{3}\right)$.

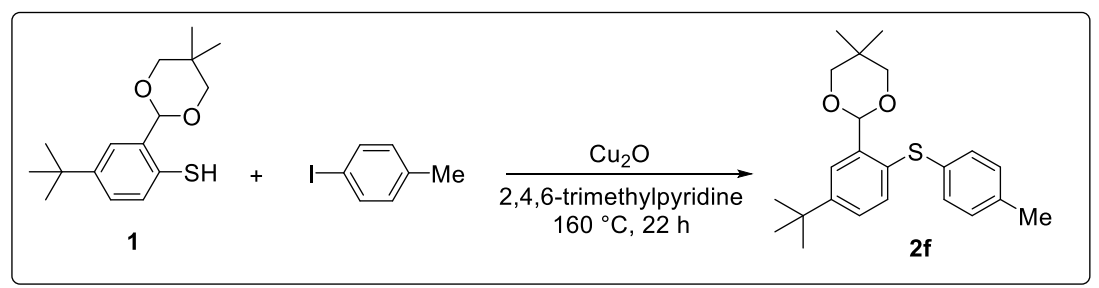

Synthesis of 2-[(5-tert-Butyl)-2-(4-methylphenylthio)phenyl]-5,5-dimethyl-1,3-dioxane 2f. Benzenethiol 1 (1.517 g, $5.41 \mathrm{mmol})$, 1-iodo-4-methylbenzene (0.840 g, $4.91 \mathrm{mmol})$, and copper(I) oxide (1.164 g, $8.13 \mathrm{mmol})$ were dissolved in 2,4,6-trimethylpyridine (30 mL). After being stirred at $160{ }^{\circ} \mathrm{C}$ for $22 \mathrm{~h}$ using an oil bath, the reaction mixture was allowed to cool to room temperature. After the solvent was removed under reduced pressure, purification of the crude product by column chromatography on silica gel (eluent: hexane/dichloromethane $\quad=\quad 1 / 1, \quad \mathrm{v} / \mathrm{v}) \quad$ afforded 2-[(5-tert-butyl)-2-(4-methylphenylthio)phenyl]-5,5-dimethyl-1,3-dioxane $2 \mathbf{2 f}$ (1.288 g, 3.48 mmol, 64\%) as colorless oil. 2f: ${ }^{1} \mathrm{H}$ NMR $\left(400 \mathrm{MHz}, \mathrm{CDCl}_{3}\right) \delta 7.77(\mathrm{~d}, J=2.4 \mathrm{~Hz}, 1 \mathrm{H}, \mathrm{ArH})$, $7.25(\mathrm{dd}, J=2.4,8.4 \mathrm{~Hz}, 1 \mathrm{H}, \mathrm{ArH}), 7.20(\mathrm{~d}, J=8.0 \mathrm{~Hz}, 2 \mathrm{H}, \mathrm{ArH}), 7.15(\mathrm{~d}, J=8.4 \mathrm{~Hz}, 1 \mathrm{H}$, ArH), $7.08(\mathrm{~d}, J=8.0 \mathrm{~Hz}, 2 \mathrm{H}, \mathrm{ArH}), 5.84(\mathrm{~s}, 1 \mathrm{H}, \mathrm{CH}), 3.74\left(\mathrm{~d}, J=11.2 \mathrm{~Hz}, 2 \mathrm{H}, \mathrm{CH}_{2}\right), 3.66(\mathrm{~d}$, 
$\left.J=11.2 \mathrm{~Hz}, 2 \mathrm{H}, \mathrm{CH}_{2}\right), 2.31\left(\mathrm{~s}, 3 \mathrm{H}, \mathrm{CH}_{3}\right), 1.32\left(\mathrm{~s}, 3 \mathrm{H}, \mathrm{CH}_{3}\right), 1.32\left(\mathrm{~s}, 9 \mathrm{H}, \mathrm{CH}_{3}\right), 0.78(\mathrm{~s}, 3 \mathrm{H}$, $\left.\mathrm{CH}_{3}\right) ;{ }^{13} \mathrm{C}\left\{{ }^{1} \mathrm{H}\right\}$ NMR $\left(101 \mathrm{MHz}, \mathrm{CDCl}_{3}\right) \delta 150.7(\mathrm{C}), 138.2(\mathrm{C}), 136.7$ (C), $132.9(\mathrm{C}), 132.2$ $(\mathrm{CH}), 131.00(\mathrm{CH}), 130.96(\mathrm{C}), 129.8(\mathrm{CH}), 126.8(\mathrm{CH}), 123.3(\mathrm{CH}), 99.8(\mathrm{CH}), 77.7\left(\mathrm{CH}_{2}\right)$, $34.7(\mathrm{C}), 31.2\left(\mathrm{CH}_{3}\right), 30.2(\mathrm{C}), 23.2\left(\mathrm{CH}_{3}\right), 21.8\left(\mathrm{CH}_{3}\right), 21.0\left(\mathrm{CH}_{3}\right)$; MS (EI, positive mode): $m / z 370\left([\mathrm{M}]^{+}\right)$; Anal. Calcd for $\mathrm{C}_{23} \mathrm{H}_{30} \mathrm{O}_{2} \mathrm{~S}: \mathrm{C}, 74.55 ; \mathrm{H}, 8.16 \%$; found: C, 74.21; H, 7.99\%.

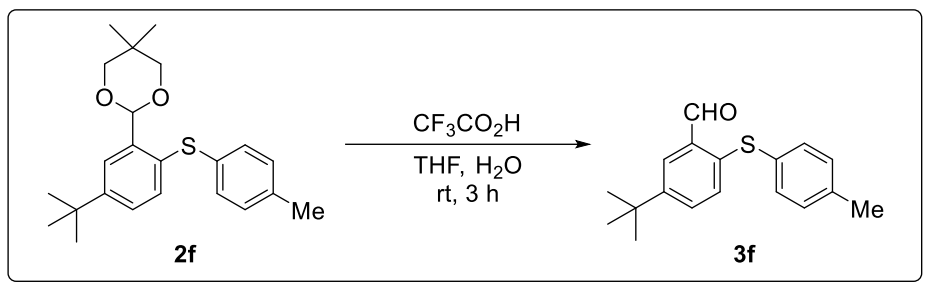

Synthesis of 5-tert-Butyl-2-(4-tolylthio)benzaldehyde 3f. Compound $2 \mathrm{f}$ ( 0.300 g, 0.810 $\mathrm{mmol})$ and trifluoroacetic acid $(5.0 \mathrm{~mL})$ was dissolved in a solution of THF $(5 \mathrm{~mL})$ and water (5 mL). After being stirred at room temperature for $3 \mathrm{~h}$, the solution was extracted with dichloromethane. After the organic layer was washed with water, the organic layers were dried over anhydrous sodium sulfate, filtered, and dried under reduced pressure to give crude yellow oil. Purification of the crude oil by column chromatography on silica gel (eluent: hexane/dichloromethane $=1 / 1, \mathrm{v} / \mathrm{v})$ afforded 5-tert-butyl-2-(4-tolylthio)benzaldehyde $\mathbf{3 f}$ (0.175 g, 0.617 mmol, 77\%) as pale yellow oil. 3f: ${ }^{1} \mathrm{H}$ NMR (400 MHz, $\left.\mathrm{CDCl}_{3}\right) \delta 10.41(\mathrm{~s}$, 1H, CH), $7.88(\mathrm{~d}, J=2.4 \mathrm{~Hz}, 1 \mathrm{H}, \mathrm{ArH}), 7.44(\mathrm{dd}, J=2.4,8.4 \mathrm{~Hz}, \mathrm{ArH}), 7.32$ (d, $J=8.0 \mathrm{~Hz}$, 2H, ArH), $7.18(\mathrm{~d}, J=8.0 \mathrm{~Hz}, 2 \mathrm{H}, \mathrm{ArH}), 7.04(\mathrm{~d}, J=8.4 \mathrm{~Hz}, 1 \mathrm{H}, \mathrm{ArH}), 2.36\left(\mathrm{~s}, 3 \mathrm{H}, \mathrm{CH}_{3}\right)$, $1.32\left(\mathrm{~s}, 9 \mathrm{H}, \mathrm{CH}_{3}\right) ;{ }^{13} \mathrm{C}\left\{{ }^{1} \mathrm{H}\right\} \mathrm{NMR}\left(101 \mathrm{MHz}, \mathrm{CDCl}_{3}\right) \delta 191.8(\mathrm{CH}), 149.7(\mathrm{C}), 138.8(\mathrm{C})$, $138.4(\mathrm{C}), 133.4(\mathrm{C}), 133.2(\mathrm{CH} \times 2), 131.5(\mathrm{CH}), 130.4(\mathrm{CH} \times 2), 130.3(\mathrm{CH}), 130.0(\mathrm{C}), 128.2$ (CH), $34.5(\mathrm{C}), 31.1\left(\mathrm{CH}_{3}\right), 21.2\left(\mathrm{CH}_{3}\right)$; MS (EI, positive mode): $m / z 284\left([\mathrm{M}]^{+}\right)$; Anal. Calcd for $\mathrm{C}_{18} \mathrm{H}_{20} \mathrm{OS}$ : C, 76.01; H, 7.09\%; found: C, 76.21; H, 7.18\%.

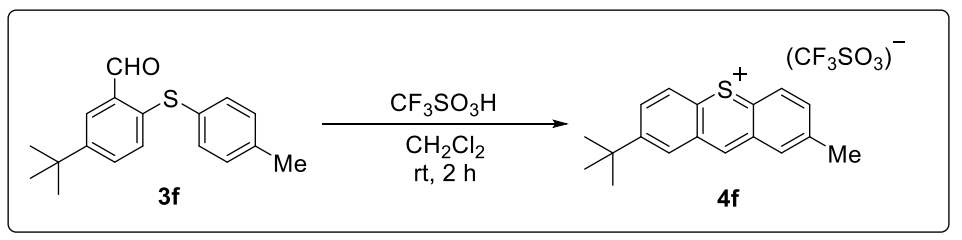


Reaction of 5-tert-Butyl-2-(4-tolylthio)benzaldehyde $3 f$ with TfOH. Trifluoromethanesulfonic acid $(0.029 \mathrm{~g}, 0.28 \mathrm{mmol})$ was added to a solution of compound $\mathbf{3 f}$ $(0.150 \mathrm{~g}, 0.527 \mathrm{mmol})$ in dichloromethane $(5 \mathrm{~mL})$ at room temperature. After the solution was stirred at room temperature for $2 \mathrm{~h}$, all volatile substances were removed under reduced pressure. The resulting red oil was washed with cold dichloromethane, and dried under reduced pressure to give 2-tert-butyl-7-methylthioxanthylium trifluoromethanesulfonate $\mathbf{4 f}$ (0.178 g, $0.427 \mathrm{mmol}, 81 \%)$ as red crystals. 4f: $\mathrm{mp} 162-164{ }^{\circ} \mathrm{C} ;{ }^{1} \mathrm{H}$ NMR $(400 \mathrm{MHz}$, $\left.\mathrm{CD}_{3} \mathrm{CN}\right) \delta 10.08(\mathrm{~s}, 1 \mathrm{H}, \mathrm{ArH}), 8.77(\mathrm{~d}, J=2.0 \mathrm{~Hz}, 1 \mathrm{H}, \mathrm{ArH}), 8.70(\mathrm{~d}, J=9.0 \mathrm{~Hz}, 1 \mathrm{H}, \mathrm{ArH})$, $8.66(\mathrm{~d}, J=8.8 \mathrm{~Hz}, 1 \mathrm{H}, \mathrm{ArH}), 8.62-8.61(\mathrm{~m}, 1 \mathrm{H}, \mathrm{ArH}), 8.55(\mathrm{dd}, J=2.0,9.0 \mathrm{~Hz}, 1 \mathrm{H}, \mathrm{ArH})$, $8.27(\mathrm{dd}, J=2.0,8.8 \mathrm{~Hz}, 1 \mathrm{H}, \mathrm{ArH}), 2.75\left(\mathrm{~s}, 3 \mathrm{H}, \mathrm{CH}_{3}\right), 1.52\left(\mathrm{~s}, 9 \mathrm{H}, \mathrm{CH}_{3}\right) ;{ }^{13} \mathrm{C}\left\{{ }^{1} \mathrm{H}\right\} \mathrm{NMR}(101$ $\left.\mathrm{MHz}, \mathrm{CD}_{3} \mathrm{CN}\right) \delta 160.4(\mathrm{CH}), 155.9(\mathrm{C}), 147.6(\mathrm{C}), 147.4(\mathrm{C}), 143.6(\mathrm{C}), 141.7(\mathrm{CH}), 138.9$ $(\mathrm{CH}), 136.3(\mathrm{CH}), 132.9(\mathrm{CH}), 131.61(\mathrm{C}), 131.59(\mathrm{C}), 128.2(\mathrm{CH}), 128.1(\mathrm{CH}), 122.1$ (quart, $\left.J_{\mathrm{CF}}=322 \mathrm{~Hz}, \mathrm{CF}_{3}\right), 36.5(\mathrm{C}), 30.9\left(\mathrm{CH}_{3}\right), 21.7\left(\mathrm{CH}_{3}\right) ;{ }^{19} \mathrm{~F}$ NMR $\left(376 \mathrm{MHz}, \mathrm{CD}_{3} \mathrm{CN}\right) \delta-79.5$ (s); MS (ESI-TOF, positive mode): $m / z 267$ ([M-OTf $\left.]^{+}\right)$; Anal. Calcd for $\mathrm{C}_{19} \mathrm{H}_{19} \mathrm{~F}_{3} \mathrm{O}_{3} \mathrm{~S}_{2}$ : C, 54.80; H, 4.60\%; found: C, 54.59; H, 4.53\%.

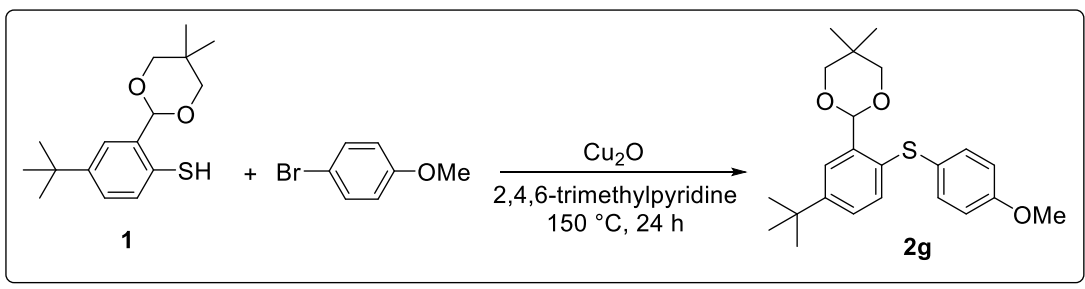

Synthesis of 2-[(5-tert-Butyl)-2-(4-methoxyphenylthio)phenyl]-5,5-dimethyl-1,3-dioxane 2g. Benzenethiol 1 (1.301 g, $4.64 \mathrm{mmol})$, 1-bomo-4-methoxybenzene (0.955 g, $5.58 \mathrm{mmol})$, and copper(I) oxide (1.294 g, $9.04 \mathrm{mmol})$ were dissolved in 2,4,6-trimethylpyridine (20 mL). After being stirred at $150{ }^{\circ} \mathrm{C}$ for $24 \mathrm{~h}$ using an oil bath, the reaction mixture was allowed to cool to room temperature. After the solvent was removed under reduced pressure, purification of the crude product by column chromatography on silica gel (eluent: hexane/dichloromethane $\quad=\quad 3 / 1, \quad \mathrm{v} / \mathrm{v}) \quad$ afforded 2-[(5-tert-butyl)-2-(4-methoxyphenylthio)phenyl]-5,5-dimethyl-1,3-dioxane 2g (1.006 g, 2.60 mmol, 58\%) as colorless crystals. 2g: mp 138.0-138.7 ${ }^{\circ} \mathrm{C} ;{ }^{1} \mathrm{H}$ NMR (400 MHz, $\left.\mathrm{CDCl}_{3}\right) \delta 7.74$ 
$(\mathrm{d}, J=2.4 \mathrm{~Hz}, 1 \mathrm{H}, \mathrm{ArH}), 7.32(\mathrm{~d}, J=8.8 \mathrm{~Hz}, 2 \mathrm{H}, \mathrm{ArH}), 7.22(\mathrm{dd}, J=2.4,8.0 \mathrm{~Hz}, 1 \mathrm{H}, \mathrm{ArH})$, $7.01(\mathrm{~d}, J=8.0 \mathrm{~Hz}, 1 \mathrm{H}, \mathrm{ArH}), 6.85(\mathrm{~d}, J=8.8 \mathrm{~Hz}, 2 \mathrm{H}, \mathrm{ArH}), 5.83$ (s, 1H, CH), 3.79 (s, 3H, $\left.\mathrm{OCH}_{3}\right), 3.76\left(\mathrm{~d}, J=10.8 \mathrm{~Hz}, 2 \mathrm{H}, \mathrm{CH}_{2}\right), 3.68\left(\mathrm{~d}, J=10.8 \mathrm{~Hz}, 2 \mathrm{H}, \mathrm{CH}_{2}\right), 1.33\left(\mathrm{~s}, 3 \mathrm{H}, \mathrm{CH}_{3}\right)$, $1.30\left(\mathrm{~s}, 9 \mathrm{H}, \mathrm{CH}_{3}\right), 0.80\left(\mathrm{~s}, 3 \mathrm{H}, \mathrm{CH}_{3}\right) ;{ }^{13} \mathrm{C}\left\{{ }^{1} \mathrm{H}\right\} \mathrm{NMR}\left(101 \mathrm{MHz}, \mathrm{CDCl}_{3}\right) \delta 159.3(\mathrm{C}), 149.9$ (C), $136.9(\mathrm{C}), 134.1(\mathrm{C}), 132.6(\mathrm{C}), 130.5(\mathrm{CH}), 126.7(\mathrm{CH}), 126.0(\mathrm{C}), 123.1(\mathrm{CH}), 114.8$ $(\mathrm{CH}), 99.6(\mathrm{CH}), 77.7\left(\mathrm{CH}_{2}\right), 55.3(\mathrm{CH}), 34.6(\mathrm{C}), 31.2\left(\mathrm{CH}_{3}\right), 30.3(\mathrm{C}), 23.2\left(\mathrm{CH}_{3}\right), 21.9$ $\left(\mathrm{CH}_{3}\right)$; MS (EI, positive mode): $\mathrm{m} / z 386\left([\mathrm{M}]^{+}\right)$; Anal. Calcd for $\mathrm{C}_{23} \mathrm{H}_{30} \mathrm{O}_{3} \mathrm{~S}: \mathrm{C}, 71.47 ; \mathrm{H}$, $7.82 \%$; found: C, $71.55 ; \mathrm{H}, 7.98 \%$.

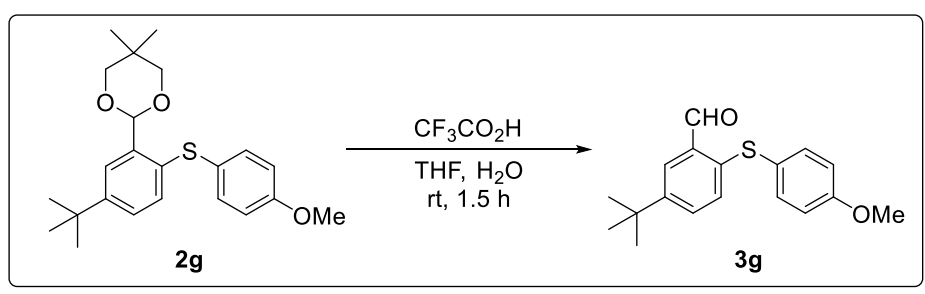

Synthesis of 5-tert-Butyl-2-(4-methoxyphenylthio)benzaldehyde 3g. Compound 2g (0.743 $\mathrm{g}, 2.10 \mathrm{mmol})$ and trifluoroacetic acid $(5.0 \mathrm{~mL})$ was dissolved in a solution of THF $(15 \mathrm{~mL})$ and water $(9 \mathrm{~mL})$. After being stirred at room temperature for $1.5 \mathrm{~h}$, the solution was extracted with dichloromethane. After the organic layer was washed with water, the organic layers were dried over anhydrous sodium sulfate, filtered, and dried under reduced pressure to give crude yellow oil. Purification of the crude oil by column chromatography on silica gel (eluent: hexane/dichloromethane $\quad=\quad 1 / 1, \quad \mathrm{v} / \mathrm{v}) \quad$ afforded 5-tert-butyl-2-(4-methoxyphenylthio)benzaldehyde $3 \mathrm{~g}(0.419 \mathrm{~g}, 1.39 \mathrm{mmol}, 66 \%)$ as pale yellow oil. 3g: ${ }^{1} \mathrm{H}$ NMR (400 MHz, $\left.\mathrm{CDCl}_{3}\right) \delta 10.37$ (s, 1H, CHO), 7.85 (d, $J=2.4 \mathrm{~Hz}, 1 \mathrm{H}$, ArH), $7.41(\mathrm{~d}, J=8.9 \mathrm{~Hz}, 2 \mathrm{H}, \mathrm{ArH}), 7.39$ (dd, $J=8.8,2.4 \mathrm{~Hz}, 1 \mathrm{H}, \mathrm{ArH}), 6.92$ (d, $J=8.9 \mathrm{~Hz}$, $2 \mathrm{H}, \mathrm{ArH}), 6.90(\mathrm{~d}, J=8.8 \mathrm{~Hz}, 1 \mathrm{H}, \mathrm{ArH}), 3.82\left(\mathrm{~s}, 3 \mathrm{H}, \mathrm{OCH}_{3}\right), 1.30\left(\mathrm{~s}, 9 \mathrm{H}, \mathrm{CH}_{3}\right) ;{ }^{13} \mathrm{C}\left\{{ }^{1} \mathrm{H}\right\}$ NMR (101 MHz, $\left.\mathrm{CDCl}_{3}\right) \delta 191.5(\mathrm{CH}), 160.2(\mathrm{C}), 148.6(\mathrm{C}), 140.3(\mathrm{C}), 136.0(\mathrm{CH}), 132.4$ (C), $131.3(\mathrm{CH}), 128.8(\mathrm{CH}), 128.5(\mathrm{CH}), 122.8(\mathrm{C}), 115.2(\mathrm{CH}), 55.2(\mathrm{CH}), 34.3(\mathrm{C}), 31.0$ $\left(\mathrm{CH}_{3}\right)$; MS (EI, positive mode): $\mathrm{m} / \mathrm{z} 300\left([\mathrm{M}]^{+}\right)$; HRMS (EI, positive mode): $\mathrm{m} / \mathrm{z}$ found $300.1192\left([\mathrm{M}]^{+}\right)$, calcd for $\mathrm{C}_{18} \mathrm{H}_{20} \mathrm{O}_{2} \mathrm{~S}: 300.1184$. 


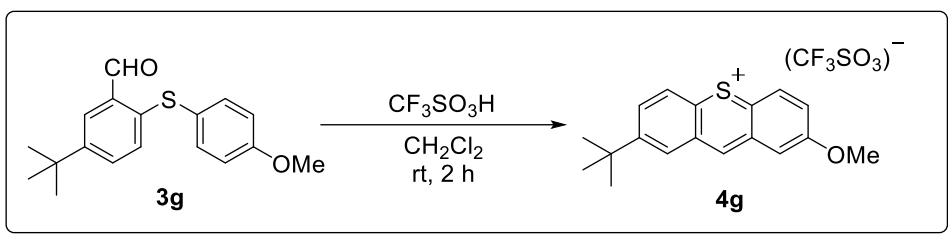

\section{Reaction of 5-tert-Butyl-2-(4-methoxyphenylthio)benzaldehyde $3 g$ with TfOH.}

Trifluoromethanesulfonic acid $(0.420 \mathrm{~g}, 2.80 \mathrm{mmol})$ was added to a solution of compound $\mathbf{3 g}$ $(0.419 \mathrm{~g}, 1.39 \mathrm{mmol})$ in dichloromethane $(10 \mathrm{~mL})$ at room temperature. After the solution was stirred at room temperature for $2 \mathrm{~h}$, all volatile substances were removed under reduced pressure. The resulting red oil was washed with cold dichloromethane, and dried under reduced pressure to give 2-tert-butyl-7-methoxythioxanthylium trifluoromethanesulfonate $\mathbf{4 g}$ (0.554 g, $1.28 \mathrm{mmol}, 92 \%)$ as red waxy solids. 4g: ${ }^{1} \mathrm{H} \mathrm{NMR}\left(400 \mathrm{MHz}, \mathrm{CD}_{3} \mathrm{CN}\right) \delta 9.98$ (s, 1H, ArH), 8.69 (d, $J=2.0 \mathrm{~Hz}, 1 \mathrm{H}, \operatorname{ArH}), 8.66(\mathrm{~d}, J=9.0 \mathrm{~Hz}, 1 \mathrm{H}, \mathrm{ArH}), 8.64(\mathrm{~d}, J=9.2 \mathrm{~Hz}$, 1H, ArH), 8.49 (dd, $J=2.0,9.0 \mathrm{~Hz}, 1 \mathrm{H}, \mathrm{ArH}), 8.11(\mathrm{~d}, J=2.7 \mathrm{~Hz}, 1 \mathrm{H}, \mathrm{ArH}), 8.02$ (dd, $J=2.7$, $9.2 \mathrm{~Hz}, 1 \mathrm{H}, \mathrm{ArH}), 4.10\left(\mathrm{~s}, 3 \mathrm{H}, \mathrm{OCH}_{3}\right), 1.51\left(\mathrm{~s}, 9 \mathrm{H}, \mathrm{CH}_{3}\right) ;{ }^{13} \mathrm{C}\left\{{ }^{1} \mathrm{H}\right\} \mathrm{NMR}\left(101 \mathrm{MHz}, \mathrm{CD}_{3} \mathrm{CN}\right)$ $\delta 162.1(\mathrm{C}), 158.2(\mathrm{CH}), 155.9(\mathrm{C}), 146.7(\mathrm{C}), 144.2(\mathrm{C}), 138.1(\mathrm{CH}), 133.7(\mathrm{C}), 132.5(\mathrm{CH})$, $132.1(\mathrm{CH}), 131.7(\mathrm{C}), 129.7(\mathrm{CH}), 128.1(\mathrm{CH}), 121.5$ (quart, $\left.{ }^{1} J_{\mathrm{CF}}=319 \mathrm{~Hz}, \mathrm{CF}_{3}\right), 114.0(\mathrm{CH})$, $57.5(\mathrm{CH}), 36.5(\mathrm{C}), 30.8(\mathrm{CH}) ;{ }^{19} \mathrm{~F}$ NMR $\left(376 \mathrm{MHz}, \mathrm{CD}_{3} \mathrm{CN}\right) \delta-79.4$ (s); MS (ESI-TOF, positive mode): $m / z, 283\left([\mathrm{M}-\mathrm{OTf}]^{+}\right)$; Anal. Calcd for $\mathrm{C}_{19} \mathrm{H}_{19} \mathrm{~F}_{3} \mathrm{O}_{4} \mathrm{~S}_{2}: \mathrm{C}, 52.77 ; \mathrm{H}, 4.43 \%$; found: C, 52.99; H, 4.67\%.

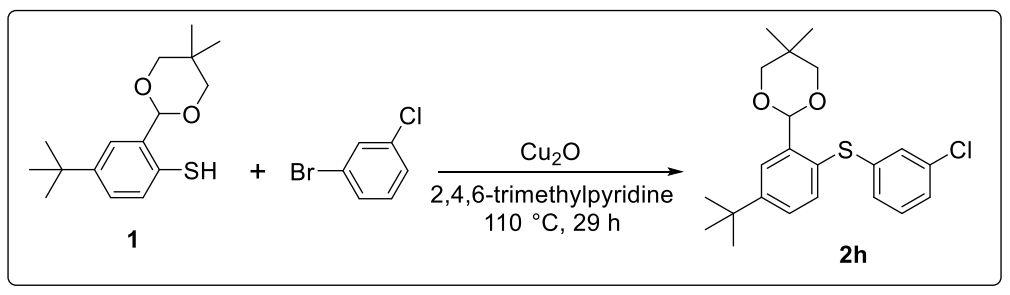

Synthesis of 2-[(5-tert-Butyl)-2-(3-chlorophenylthio)phenyl]-5,5-dimethyl-1,3-dioxane $2 \mathrm{~h}$. Benzenethiol 1 (1.077 g, $3.84 \mathrm{mmol}$ ), 1-bromo-3-chlorobenzene (0.902 g, $4.71 \mathrm{mmol})$, and copper(I) oxide (1.104 g, $7.72 \mathrm{mmol})$ were dissolved in 2,4,6-trimethylpyridine (13 mL). After being stirred at $110{ }^{\circ} \mathrm{C}$ for $29 \mathrm{~h}$ using an oil bath, the reaction mixture was allowed to 
cool to room temperature. After the solvent was removed under reduced pressure, purification of the crude product by column chromatography on silica gel (eluent: $\begin{array}{lllll}\text { dichloromethane/hexane } \quad=\quad 1 / 1, & \mathrm{v} / \mathrm{v}) \quad \text { afforded }\end{array}$ 2-[(5-tert-butyl)-2-(3-chlorophenylthio)phenyl]-5,5-dimethyl-1,3-dioxane $\mathbf{2 h}(0.455 \mathrm{~g}, 1.16$ mmol, 30\%) as colorless oil. 2h: ${ }^{1} \mathrm{H}$ NMR $\left(400 \mathrm{MHz}, \mathrm{CDCl}_{3}\right) \delta 7.82(\mathrm{~d}, J=2.4 \mathrm{~Hz}, 1 \mathrm{H}, \mathrm{ArH})$, $7.35(\mathrm{dd}, J=2.4,8.0 \mathrm{~Hz}, 1 \mathrm{H}, \operatorname{ArH}), 7.31(\mathrm{~d}, J=8.0 \mathrm{~Hz}, 1 \mathrm{H}, \operatorname{ArH}), 7.22(\mathrm{~s}, 1 \mathrm{H}, \operatorname{ArH})$, 7.17-7.10 (m, 2H, ArH), $7.03(\mathrm{~d}, J=7.2 \mathrm{~Hz}, 1 \mathrm{H}, \mathrm{ArH}), 5.81(\mathrm{~s}, 1 \mathrm{H}, \mathrm{CH}), 3.72(\mathrm{~d}, J=10.8 \mathrm{~Hz}$, 2H, $\left.\mathrm{CH}_{2}\right), 3.62\left(\mathrm{~d}, J=10.8 \mathrm{~Hz}, 2 \mathrm{H}, \mathrm{CH}_{2}\right), 1.34\left(\mathrm{~s}, 9 \mathrm{H}, \mathrm{CH}_{3}\right), 1.32\left(\mathrm{~s}, 3 \mathrm{H}, \mathrm{CH}_{3}\right), 0.78(\mathrm{~s}, 3 \mathrm{H}$, $\left.\mathrm{CH}_{3}\right) ;{ }^{13} \mathrm{C}\left\{{ }^{1} \mathrm{H}\right\}$ NMR (101 MHz, $\left.\mathrm{CDCl}_{3}\right) \delta 152.4(\mathrm{C}), 139.9(\mathrm{C}), 139.8$ (C), 134.7 (C), 134.2 $(\mathrm{CH}), 129.9(\mathrm{CH}), 128.6(\mathrm{CH}), 128.1(\mathrm{C}), 127.2(\mathrm{CH}), 127.1(\mathrm{CH}), 126.1(\mathrm{CH}), 123.9(\mathrm{CH})$, $99.8(\mathrm{CH}), 77.8\left(\mathrm{CH}_{2}\right), 34.9(\mathrm{C}), 31.2\left(\mathrm{CH}_{3}\right), 30.3(\mathrm{C}), 23.2\left(\mathrm{CH}_{3}\right), 21.8\left(\mathrm{CH}_{3}\right) ;$ MS (ESI-TOF, positive mode): $\mathrm{m} / \mathrm{z} 413\left([\mathrm{M}+\mathrm{Na}]^{+}\right)$; Anal. Calcd for $\mathrm{C}_{22} \mathrm{H}_{27} \mathrm{ClO}_{2} \mathrm{~S}: \mathrm{C}, 67.59 ; \mathrm{H}, 6.96 \%$; found: C, 67.35; H, 6.96\%.

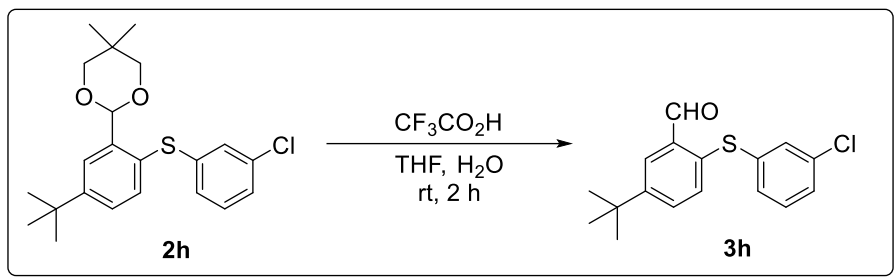

Synthesis of 5-tert-Butyl-2-(3-chlorophenylthio)benzaldehyde 3h. Compound $\mathbf{2 h}(0.295 \mathrm{~g}$, $0.755 \mathrm{mmol})$ and trifluoroacetic acid $(5.0 \mathrm{~mL})$ was dissolved in a solution of THF $(5 \mathrm{~mL})$ and water $(5 \mathrm{~mL})$. After being stirred at room temperature for $2 \mathrm{~h}$, the solution was extracted with dichloromethane. After the organic layer was washed with water, the organic layers were dried over anhydrous sodium sulfate, filtered, and dried under reduced pressure to give crude yellow oil. Purification of the crude oil by column chromatography on silica gel $\begin{array}{llll}\text { (hexane/dichloromethane } & = & 1 / 1, & \mathrm{v} / \mathrm{v})\end{array}$ 5-tert-butyl-2-(3-chlorophenylthio)benzaldehyde $\mathbf{3 h}(0.142 \mathrm{~g}, 0.466 \mathrm{mmol}, 62 \%)$ as yellow oil. 3h: ${ }^{1} \mathrm{H}$ NMR (400 MHz, $\left.\mathrm{CDCl}_{3}\right) \delta 10.42(\mathrm{~s}, 1 \mathrm{H}, \mathrm{CH}), 7.95(\mathrm{~d}, J=2.4 \mathrm{~Hz}, 1 \mathrm{H}, \mathrm{ArH}), 7.54$ $(\mathrm{dd}, J=2.4,8.4 \mathrm{~Hz}, 1 \mathrm{H}, \mathrm{ArH}), 7.32(\mathrm{~s}, 1 \mathrm{H}, \mathrm{ArH}), 7.26(\mathrm{~d}, J=8.4 \mathrm{~Hz}, 1 \mathrm{H}, \mathrm{ArH}), 7.21-7.19(\mathrm{~m}$, 2H, ArH), $1.35\left(\mathrm{~s}, 9 \mathrm{H}, \mathrm{CH}_{3}\right) ;{ }^{13} \mathrm{C}\left\{{ }^{1} \mathrm{H}\right\} \mathrm{NMR}\left(101 \mathrm{MHz}, \mathrm{CDCl}_{3}\right) \delta 191.8(\mathrm{CH}), 151.3(\mathrm{C})$, 
$137.0(\mathrm{C}), 135.6(\mathrm{C}), 135.2(\mathrm{C}), 134.5(\mathrm{C}), 132.4(\mathrm{CH}), 131.9(\mathrm{CH}), 131.0(\mathrm{CH}), 130.5(\mathrm{CH})$, $129.4(\mathrm{CH}), 128.1(\mathrm{CH}), 127.8(\mathrm{CH}), 34.8(\mathrm{C}), 31.1\left(\mathrm{CH}_{3}\right)$; MS (EI, positive mode): $\mathrm{m} / z .304$ $\left([\mathrm{M}]^{+}\right)$; Anal. Calcd for $\mathrm{C}_{17} \mathrm{H}_{27} \mathrm{ClOS}: \mathrm{C}, 66.98 ; \mathrm{H}, 5.62 \%$; found: $\mathrm{C}, 66.85 ; \mathrm{H}, 5.81 \%$.

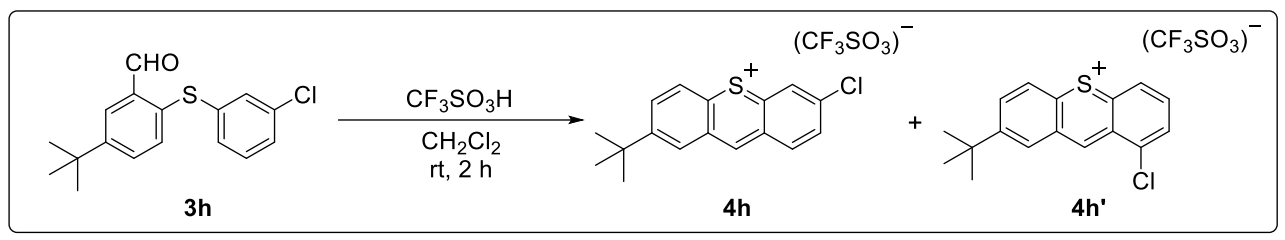

\section{Reaction of 5-tert-Butyl-2-(3-chlorophenylthio)benzaldehyde $3 \mathrm{~h}$ with TfOH.}

Trifluoromethanesulfonic acid $(0.099 \mathrm{~g}, 0.66 \mathrm{mmol})$ was added to a solution of compound $\mathbf{3 h}$ $(0.101 \mathrm{~g}, 0.331 \mathrm{mmol})$ in dichloromethane $(6 \mathrm{~mL})$ at room temperature. After the solution was stirred at room temperature for $2 \mathrm{~h}$, all volatile substances were removed under reduced pressure. The formation of 2-tert-butyl-6-chlorothioxanthylium trifluoromethanesulfonate $\mathbf{4 h}$ and 2-tert-butyl-8-chlorothioxanthylium trifluoromethanesulfonate $\mathbf{4 h}^{\mathbf{\prime}}$ was detected by NMR

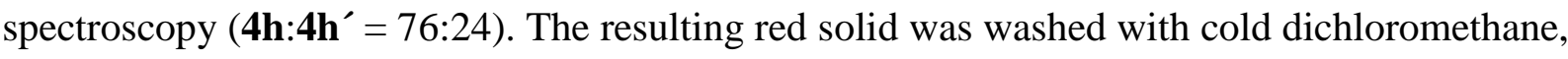
and dried under reduced pressure to give $\mathbf{4 h}$ and $\mathbf{4 h}^{\mathbf{\prime}}(0.114 \mathrm{~g}, 0.261 \mathrm{mmol}, 79 \%)$ as red solid. 4h and 4h': mp 210-212 ${ }^{\circ} \mathrm{C}$; MS (ESI-TOF, positive mode): $m / z 287$ ([M-OTf $]^{+}$); Anal. Calcd for $\mathrm{C}_{18} \mathrm{H}_{16} \mathrm{ClF}_{3} \mathrm{O}_{3} \mathrm{~S}_{2}$ : C, 49.49; H, 3.69\%; found: C, 49.25; H, 3.99\%. 4h: ${ }^{1} \mathrm{H}$ NMR (400 $\left.\mathrm{MHz}, \mathrm{CD}_{3} \mathrm{CN}\right) \delta 10.17(\mathrm{~s}, 1 \mathrm{H}, \mathrm{ArH}), 8.83(\mathrm{~d}, J=2.0 \mathrm{~Hz}, 1 \mathrm{H}, \mathrm{ArH}), 8.80(\mathrm{~d}, J=8.8 \mathrm{~Hz}, 1 \mathrm{H}$, $\operatorname{ArH}), 8.79(\mathrm{~d}, J=2.0 \mathrm{~Hz}, 1 \mathrm{H}, \mathrm{ArH}), 8.73$ (d, $J=9.0 \mathrm{~Hz}, 1 \mathrm{H}, \operatorname{ArH}), 8.61(\mathrm{dd}, J=2.0,9.0 \mathrm{~Hz}$, $1 \mathrm{H}, \mathrm{ArH}), 8.16(\mathrm{dd}, J=2.0,8.8 \mathrm{~Hz}, 1 \mathrm{H}, \mathrm{ArH}), 1.52\left(\mathrm{~s}, 9 \mathrm{H}, \mathrm{CH}_{3}\right) ;{ }^{13} \mathrm{C}\left\{{ }^{1} \mathrm{H}\right\} \mathrm{NMR}(101 \mathrm{MHz}$, $\left.\mathrm{CD}_{3} \mathrm{CN}\right) \delta 162.1(\mathrm{C}), 158.2(\mathrm{CH}), 155.9(\mathrm{C}), 146.7(\mathrm{C}), 144.2(\mathrm{C}), 138.1(\mathrm{CH}), 133.7(\mathrm{C})$, $132.5(\mathrm{CH}), 132.1(\mathrm{CH}), 131.7(\mathrm{C}), 129.7(\mathrm{CH}), 128.1(\mathrm{CH}), 121.5$ (quart, $J=320 \mathrm{~Hz}, \mathrm{CF}_{3}$ ), $114.0(\mathrm{CH}), 36.5(\mathrm{C}), 30.8\left(\mathrm{CH}_{3}\right) .4 \mathbf{h}^{\prime}:{ }^{1} \mathrm{H}$ NMR (400 MHz, $\left.\mathrm{CD}_{3} \mathrm{CN}\right) \delta 10.58(\mathrm{~s}, 1 \mathrm{H}, \mathrm{ArH})$, $9.00(\mathrm{~s}, 1 \mathrm{H}, \mathrm{ArH}), 8.78(\mathrm{~d}, J=7.2 \mathrm{~Hz}, 1 \mathrm{H}, \operatorname{ArH}), 8.69$ (d, $J=7.2 \mathrm{~Hz}, 1 \mathrm{H}, \operatorname{ArH}), 8.65$ (d, $J=$ $7.2 \mathrm{~Hz}, 1 \mathrm{H}, \mathrm{ArH}), 8.31(\mathrm{~d}, J=7.2 \mathrm{~Hz}, 2 \mathrm{H}, \mathrm{ArH}), 1.53\left(\mathrm{~s}, 9 \mathrm{H}, \mathrm{CH}_{3}\right)$. 


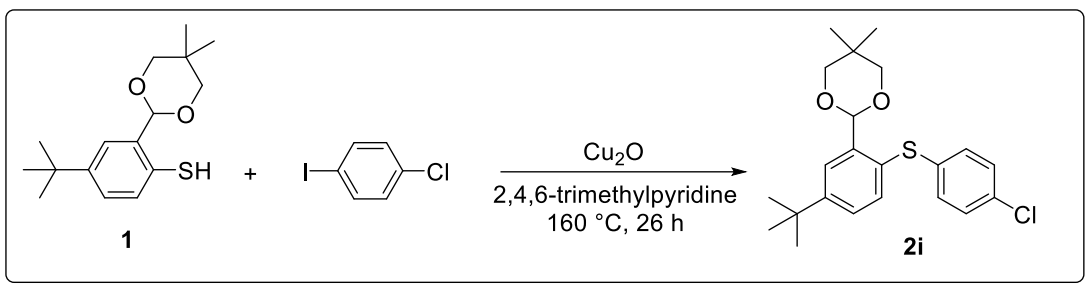

Synthesis of 2-[(5-tert-Butyl)-2-(4-chlorophenylthio)phenyl]-5,5-dimethyl-1,3-dioxane 2i.

Benzenethiol 1 (1.435 g, $5.12 \mathrm{mmol})$, 1-chloro-4-iodobenzene (1.216 g, $5.10 \mathrm{mmol})$, and copper(I) oxide (1.499 g, $10.5 \mathrm{mmol})$ were dissolved in 2,4,6-trimethylpyridine (30 mL). After being stirred at $160{ }^{\circ} \mathrm{C}$ for $26 \mathrm{~h}$ using an oil bath, the reaction mixture was allowed to cool to room temperature. After the solvent was removed under reduced pressure, purification of the crude product by column chromatography on silica gel (eluent: dichloromethane/hexane $\quad=\quad 1 / 1, \quad \mathrm{v} / \mathrm{v}) \quad$ afforded 2-[(5-tert-butyl)-2-(4-chlorophenylthio)phenyl]-5,5-dimethyl-1,3-dioxane $2 \mathbf{i}$ (0.581 g, 1.49 mmol, 29\%) as pale yellow oil. 2i: ${ }^{1} \mathrm{H}$ NMR (400 $\left.\mathrm{MHz}, \mathrm{CDCl}_{3}\right) \delta 7.81(\mathrm{~d}, J=2.4 \mathrm{~Hz}, 1 \mathrm{H}$, ArH), $7.32(\mathrm{dd}, J=2.4,8.0 \mathrm{~Hz}, 1 \mathrm{H}, \mathrm{ArH}), 7.26(\mathrm{~d}, J=8.0 \mathrm{~Hz}, 1 \mathrm{H}, \operatorname{ArH}), 7.21(\mathrm{~d}, J=8.8 \mathrm{~Hz}$, 2H, ArH), 7.14 (d, $J=8.8 \mathrm{~Hz}, 2 \mathrm{H}, \mathrm{ArH}), 5.82(\mathrm{~s}, 1 \mathrm{H}, \mathrm{CH}), 3.72\left(\mathrm{~d}, J=10.4 \mathrm{~Hz}, 2 \mathrm{H}, \mathrm{CH}_{2}\right)$, 3.62 (d, $\left.J=10.4 \mathrm{~Hz}, 2 \mathrm{H}, \mathrm{CH}_{2}\right), 1.33$ (s, 9H, $\left.\mathrm{CH}_{3}\right), 1.32$ (s, 3H, $\left.\mathrm{CH}_{3}\right), 0.77$ (s, 3H, $\left.\mathrm{CH}_{3}\right)$; ${ }^{13} \mathrm{C}\left\{{ }^{1} \mathrm{H}\right\}$ NMR (101 MHz, $\left.\mathrm{CDCl}_{3}\right) \delta 152.0(\mathrm{C}), 139.4(\mathrm{C}), 136.1(\mathrm{C}), 133.7(\mathrm{CH}), 132.1(\mathrm{C})$, $130.7(\mathrm{CH}), 129.1(\mathrm{CH}), 129.0(\mathrm{C}), 127.1(\mathrm{CH}), 123.8(\mathrm{CH}), 99.8(\mathrm{CH}), 77.8\left(\mathrm{CH}_{2}\right), 34.8(\mathrm{C})$, $31.2\left(\mathrm{CH}_{3}\right), 30.2(\mathrm{C}), 23.2\left(\mathrm{CH}_{3}\right), 21.8\left(\mathrm{CH}_{3}\right)$; MS (EI, positive mode): $m / z 390\left([\mathrm{M}]^{+}\right)$; Anal. Calcd for $\mathrm{C}_{22} \mathrm{H}_{27} \mathrm{ClO}_{2} \mathrm{~S}$ : C, 67.59; H, 6.96\%; found: C, 67.64; H, 6.86\%.

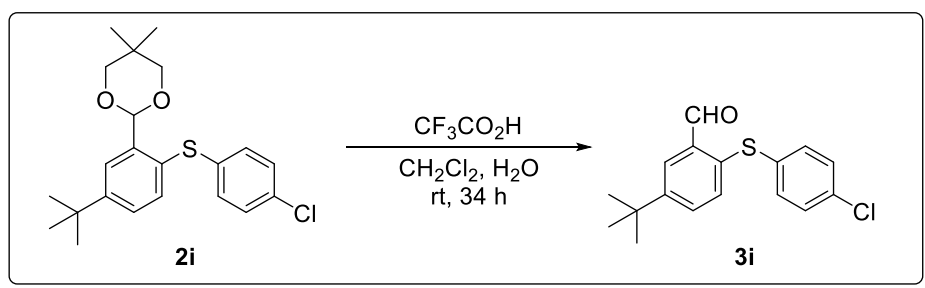

Synthesis of 5-tert-Butyl-2-(4-chlorophenylthio)benzaldehyde 3i. Compound $2 \mathrm{i}(0.300 \mathrm{~g}$, $0.767 \mathrm{mmol})$ and trifluoroacetic acid $(5.0 \mathrm{~mL})$ was dissolved in a solution of THF $(5 \mathrm{~mL})$ and 
water $(5 \mathrm{~mL})$. After being stirred at room temperature for $34 \mathrm{~h}$, the solution was extracted with dichloromethane. After the organic layer was washed with water, the organic layers were dried over anhydrous sodium sulfate, filtered, and dried under reduced pressure to give crude yellow oil. Purification of the crude oil by column chromatography on silica gel (eluent: hexane/dichloromethane $=1 / 1, \mathrm{v} / \mathrm{v}$ ) afforded 5-tert-butyl-2-(4-chlorophenylthio)benzaldehyde $3 \mathbf{i}(0.149 \mathrm{~g}, 0.489 \mathrm{mmol}, 64 \%)$ as pale yellow oil. 3i: ${ }^{1} \mathrm{H}$ NMR (400 MHz, $\left.\mathrm{CDCl}_{3}\right) \delta 10.40(\mathrm{~s}$, 1H, ArH), 7.92 (d, $J=2.4 \mathrm{~Hz}, 1 \mathrm{H}, \mathrm{ArH}), 7.49$ (dd, $J=2.4,8.4 \mathrm{~Hz}, 1 \mathrm{H}, \mathrm{ArH}), 7.34-7.26$ (m, $2 \mathrm{H}, \operatorname{ArH}), 7.11(\mathrm{~d}, J=8.4 \mathrm{~Hz}, 1 \mathrm{H}, \mathrm{ArH}), 1.34\left(\mathrm{~s}, 9 \mathrm{H}, \mathrm{CH}_{3}\right) ;{ }^{13} \mathrm{C}\left\{{ }^{1} \mathrm{H}\right\} \mathrm{NMR}(101 \mathrm{MHz}$, $\left.\mathrm{CDCl}_{3}\right) \delta 191.8(\mathrm{CH}), 150.7(\mathrm{C}), 136.8(\mathrm{C}), 134.2(\mathrm{C}), 134.0(\mathrm{C}), 133.4(\mathrm{CH}), 132.9(\mathrm{C})$, $131.7(\mathrm{CH}), 131.3(\mathrm{CH}), 129.7(\mathrm{CH}), 128.4(\mathrm{CH}), 34.7(\mathrm{C}), 31.1\left(\mathrm{CH}_{3}\right) ; \mathrm{MS}$ (EI, positive mode): $m / z 304\left([\mathrm{M}]^{+}\right)$; HRMS (EI, positive mode): $m / z$ found $304.0696\left([\mathrm{M}]^{+}\right)$, calcd for $\mathrm{C}_{17} \mathrm{H}_{17}{ }^{35} \mathrm{ClOS}: 304.0689$.

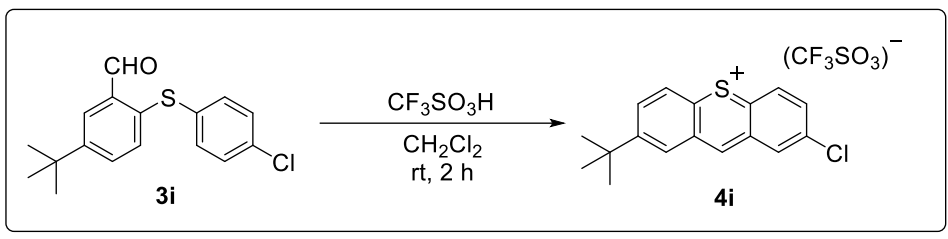

\section{Reaction of 5-tert-Butyl-2-(4-chlorophenylthio)benzaldehyde 3i with TfOH.} Trifluoromethanesulfonic acid $(0.074 \mathrm{~g}, 0.49 \mathrm{mmol})$ was added to a solution of compound $\mathbf{3 i}$ $(0.072 \mathrm{~g}, 0.24 \mathrm{mmol})$ in dichloromethane $(2 \mathrm{~mL})$ at room temperature. After the solution was stirred at room temperature for $2 \mathrm{~h}$, all volatile substances were removed under reduced pressure. The resulting red oil was washed with cold dichloromethane, and dried under reduced pressure to give 2-tert-butyl-7-chlorothioxanthylium trifluoromethanesulfonate $\mathbf{4 i}$ $(0.087 \mathrm{~g}, 0.20 \mathrm{mmol}, 83 \%)$ as red oil. ${ }^{1} \mathrm{H}$ NMR (400 MHz, $\left.\mathrm{CD}_{3} \mathrm{CN}\right) \delta 10.15(\mathrm{~s}, 1 \mathrm{H}, \mathrm{ArH})$, $8.88(\mathrm{~d}, J=2.0 \mathrm{~Hz}, 1 \mathrm{H}, \mathrm{ArH}), 8.81(\mathrm{~d}, J=2.0 \mathrm{~Hz}, 1 \mathrm{H}, \mathrm{ArH}), 8.76(\mathrm{~d}, J=9.2 \mathrm{~Hz}, 1 \mathrm{H}, \operatorname{ArH})$, $8.75(\mathrm{~d}, J=9.2 \mathrm{~Hz}, 1 \mathrm{H}, \mathrm{ArH}), 8.62(\mathrm{dd}, J=2.0,9.2 \mathrm{~Hz}, 1 \mathrm{H}, \mathrm{ArH}), 8.34(\mathrm{dd}, J=2.0,9.2 \mathrm{~Hz}$, $1 \mathrm{H}, \mathrm{ArH}), 1.52\left(\mathrm{~s}, 9 \mathrm{H}, \mathrm{CH}_{3}\right) ;{ }^{13} \mathrm{C}\left\{{ }^{1} \mathrm{H}\right\} \mathrm{NMR}\left(101 \mathrm{MHz}, \mathrm{CD}_{3} \mathrm{CN}\right) \delta 160.3(\mathrm{CH}), 156.7(\mathrm{C})$, $148.7(\mathrm{C}), 147.7(\mathrm{C}), 140.0(\mathrm{CH}), 139.1(\mathrm{CH}), 137.8(\mathrm{C}), 135.7(\mathrm{CH}), 133.2(\mathrm{CH}), 132.2(\mathrm{C})$, 
$131.9(\mathrm{C}), 130.2(\mathrm{CH}), 128.5(\mathrm{CH}), 121.4\left(\mathrm{q}, J_{\mathrm{CF}}=320 \mathrm{~Hz}, \mathrm{CF}_{3}\right), 36.6(\mathrm{C}), 30.8\left(\mathrm{CH}_{3}\right) ;{ }^{19} \mathrm{~F}$ NMR (376 MHz, CD $\left.{ }_{3} \mathrm{CN}\right) \delta-79.4$ (s); MS (ESI-TOF, positive mode): $m / z 287$ ([M-OTf $]^{+}$); HRMS (ESI-TOF, positive mode): $\mathrm{m} / z$ found $287.0655\left([\mathrm{M}-\mathrm{TfO}]^{+}\right)$, calcd for $\mathrm{C}_{17} \mathrm{H}_{16}{ }^{35} \mathrm{ClS}$ : 287.0661

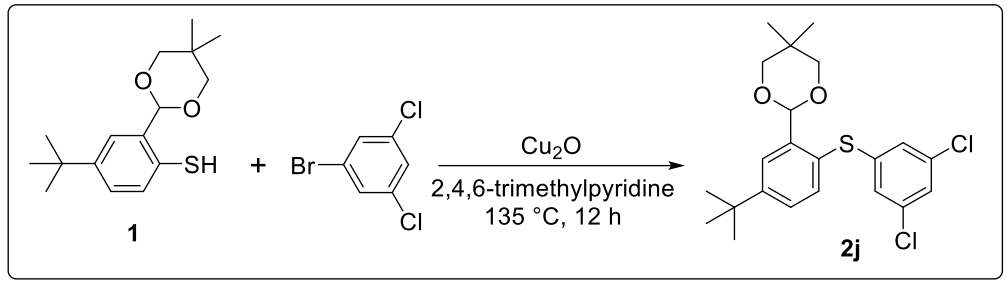

Synthesis of 2-[(5-tert-Butyl)-2-(3,5-dichlorophenylthio)phenyl]-5,5-dimethyl-1,3-dioxane 2j. Benzenethiol 1 (2.873 g, $10.2 \mathrm{mmol})$, 1-bromo-3,5-dichlorobenzene (2.755 g, $12.2 \mathrm{mmol}$ ), and copper(I) oxide $(2.919 \mathrm{~g}, 20.4 \mathrm{mmol})$ were dissolved in 2,4,6-trimethylpyridine (20 mL). After being stirred at $135{ }^{\circ} \mathrm{C}$ for $12 \mathrm{~h}$ using an oil bath, the reaction mixture was allowed to cool to room temperature. After the solvent was removed under reduced pressure, purification of the crude product by column chromatography on silica gel (eluent: dichloromethane/hexane $\quad=\quad 1 / 1, \quad \mathrm{v} / \mathrm{v}) \quad$ afforded 2-[(5-tert-butyl)-2-(3,5-dichlorophenylthio)phenyl]-5,5-dimethyl-1,3-dioxane $\quad \mathbf{2 j} \quad(0.434 \mathrm{~g}$, $1.02 \mathrm{mmol}, 10 \%)$ as colorless crystals. $2 \mathrm{j}: \mathrm{mp} 111.5-112.8{ }^{\circ} \mathrm{C} ;{ }^{1} \mathrm{H}$ NMR $\left(400 \mathrm{MHz}, \mathrm{CDCl}_{3}\right) \delta$ $7.84(\mathrm{~d}, J=2.4 \mathrm{~Hz}, 1 \mathrm{H}, \mathrm{ArH}), 7.36(\mathrm{dd}, J=2.0,8.0 \mathrm{~Hz}, 1 \mathrm{H}, \mathrm{ArH}), 7.34(\mathrm{~d}, J=8.0 \mathrm{~Hz}, 1 \mathrm{H}$, $\operatorname{ArH}), 7.12$ (s, 1H, ArH), 7.03 (s, 1H, ArH), $5.76(\mathrm{~s}, 1 \mathrm{H}, \mathrm{CH}), 3.72\left(\mathrm{~d}, J=11.2 \mathrm{~Hz}, 2 \mathrm{H}, \mathrm{CH}_{2}\right)$, $3.62\left(\mathrm{~d}, J=11.2 \mathrm{~Hz}, 2 \mathrm{H}, \mathrm{CH}_{2}\right), 1.35\left(\mathrm{~s}, 9 \mathrm{H}, \mathrm{CH}_{3}\right), 1.31\left(\mathrm{~s}, 3 \mathrm{H}, \mathrm{CH}_{3}\right), 0.78\left(\mathrm{~s}, 3 \mathrm{H}, \mathrm{CH}_{3}\right)$; ${ }^{13} \mathrm{C}\left\{{ }^{1} \mathrm{H}\right\}$ NMR $\left(101 \mathrm{MHz}, \mathrm{CDCl}_{3}\right) \delta 153.0(\mathrm{C}), 141.8(\mathrm{C}), 140.3(\mathrm{C}), 135.2(\mathrm{C}), 134.8(\mathrm{CH})$, $127.4(\mathrm{CH}), 126.7(\mathrm{CH}), 125.9(\mathrm{CH}), 124.2(\mathrm{CH}), 99.8(\mathrm{CH}), 77.2\left(\mathrm{CH}_{2}\right), 34.9(\mathrm{C}), 31.2$ $\left(\mathrm{CH}_{3}\right), 30.2(\mathrm{C}), 23.2\left(\mathrm{CH}_{3}\right), 21.8\left(\mathrm{CH}_{3}\right)$; MS (EI, positive mode): $m / 2424\left([\mathrm{M}]^{+}\right)$; Anal. Calcd for $\mathrm{C}_{22} \mathrm{H}_{26} \mathrm{Cl}_{2} \mathrm{O}_{2} \mathrm{~S}$ : C, 62.11; H, 6.16\%; found: C, 62.13; H, $6.23 \%$. 


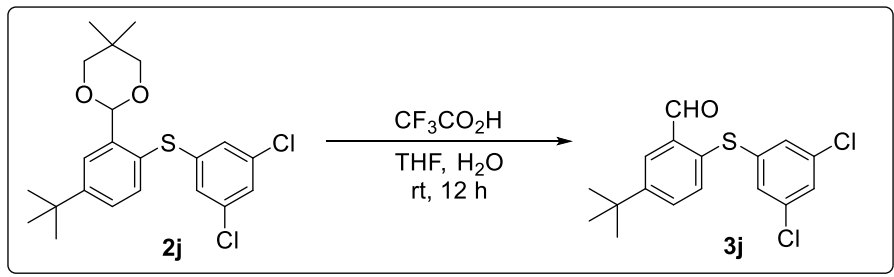

Synthesis of 5-tert-Butyl-2-(3,5-dichlorophenylthio)benzaldehyde 3j. Compound $2 \mathbf{j}$ (0.403 $\mathrm{g}, 0.947 \mathrm{mmol})$ and trifluoroacetic acid $(5.0 \mathrm{~mL})$ was dissolved in a solution of THF $(5 \mathrm{~mL})$ and water $(5 \mathrm{~mL})$. After being stirred at room temperature for $2 \mathrm{~h}$, the solution was extracted with dichloromethane. After the organic layer was washed with water, the organic layers were dried over anhydrous sodium sulfate, filtered, and dried under reduced pressure to give crude yellow oil. Purification of the crude oil by column chromatography on silica gel (hexane/dichloromethane $=\quad 1 / 1$, $\mathrm{v} / \mathrm{v})$ afforded 5-tert-butyl-2-(3,5-dichlorophenylthio)benzaldehyde $\mathbf{3 j}(0.263 \mathrm{~g}, 0.775 \mathrm{mmol}, 82 \%)$ as yellow oil. 2j: ${ }^{1} \mathrm{H}$ NMR (400 MHz, $\left.\mathrm{CDCl}_{3}\right) \delta 10.41$ (s, 1H, CHO), 7.98 (d, J = $2.4 \mathrm{~Hz}, 1 \mathrm{H}$, ArH), 7.59 (dd, $J=2.4,8.4 \mathrm{~Hz}, 1 \mathrm{H}, \mathrm{ArH}), 7.27$ (d, $J=8.0 \mathrm{~Hz}, 1 \mathrm{H}, \mathrm{ArH}), 7.25$ (d, $J=2.0 \mathrm{~Hz}$, 1H, ArH), 7.15 (d, J=2.0 Hz, 2H, ArH), $1.35\left(\mathrm{~s}, 9 \mathrm{H}, \mathrm{CH}_{3}\right) ;{ }^{13} \mathrm{C}\left\{{ }^{1} \mathrm{H}\right\} \mathrm{NMR}\left(101 \mathrm{MHz}, \mathrm{CDCl}_{3}\right)$ $\delta 191.6(\mathrm{CHO}), 152.3(\mathrm{C}), 139.2(\mathrm{C}), 135.8(\mathrm{C}), 135.0(\mathrm{C}), 133.8(\mathrm{C}), 133.3(\mathrm{C}), 132.1(\mathrm{CH})$, $128.4(\mathrm{CH}), 128.1(\mathrm{CH}), 127.5(\mathrm{CH}), 126.3(\mathrm{CH}), 34.9(\mathrm{C}), 31.0\left(\mathrm{CH}_{3}\right)$; MS (EI, positive mode): $m / z 338$ ([M] $\left.]^{+}\right)$; Anal. Calcd for $\mathrm{C}_{17} \mathrm{H}_{16} \mathrm{Cl}_{2} \mathrm{OS}: \mathrm{C}, 60.18 ; \mathrm{H}, 4.75 \%$; found: $\mathrm{C}, 59.94$. H, $5.04 \%$.

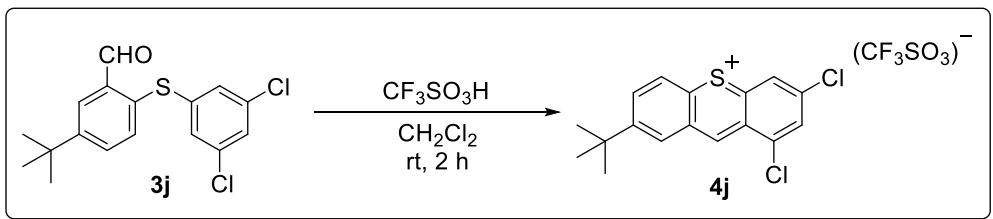

Reaction of 5-tert-Butyl-2-(3,5-dichlorophenylthio)benzaldehyde $3 \mathrm{j}$ with TfOH. Trifluoromethanesulfonic acid $(0.211 \mathrm{~g}, 1.41 \mathrm{mmol})$ was added to a solution of compound $\mathbf{3 j}$ $(0.222 \mathrm{~g}, 0.654 \mathrm{mmol})$ in dichloromethane $(5 \mathrm{~mL})$ at room temperature. After the solution was stirred at room temperature for $2 \mathrm{~h}$, the reaction mixture was added dropwise to cold $\mathrm{Et}_{2} \mathrm{O}(20$ 
$\mathrm{mL}$ ). Purification of the resulting suspension using a centrifugal separator afforded 2-tert-butyl-6,8-dichlorothioxanthylium trifluoromethanesulfonate $4 \mathbf{j}$ (0.296 g, 0.628 mmol, 96\%) as red waxy solid. 4j: ${ }^{1} \mathrm{H}$ NMR (400 MHz, $\left.\mathrm{CD}_{3} \mathrm{CN}\right) \delta 10.50(\mathrm{~s}, 1 \mathrm{H}, \mathrm{ArH}), 8.98(\mathrm{~d}, J=$ $2.0 \mathrm{~Hz}, 1 \mathrm{H}, \operatorname{ArH}), 8.77$ (d, $J=2.0 \mathrm{~Hz}, 2 \mathrm{H}, \operatorname{ArH}), 8.75(\mathrm{~d}, J=8.8 \mathrm{~Hz}, 1 \mathrm{H}, \operatorname{ArH}), 8.66$ (dd, $J=$ 2.0, $8.8 \mathrm{~Hz}, 1 \mathrm{H}, \mathrm{ArH}), 8.33(\mathrm{~d}, J=2.0 \mathrm{~Hz}, 1 \mathrm{H}, \mathrm{ArH}), 1.53\left(\mathrm{~s}, 9 \mathrm{H}, \mathrm{CH}_{3}\right) ;{ }^{13} \mathrm{C}\left\{{ }^{1} \mathrm{H}\right\} \mathrm{NMR}(101$ $\left.\mathrm{MHz}, \mathrm{CDCl}_{3}\right) \delta 157.8(\mathrm{CH}), 157.0(\mathrm{C}), 150.9(\mathrm{C}), 148.0(\mathrm{C}), 145.8$ (C), $141.6(\mathrm{C}), 140.7$ $(\mathrm{CH}), 134.3(\mathrm{CH}), 133.3(\mathrm{CH}), 131.9(\mathrm{C}), 128.2(\mathrm{CH}), 128.1(\mathrm{C}), 127.1(\mathrm{CH}), 121.72$ (quart, $J$ $\left.=320 \mathrm{~Hz}, \mathrm{CF}_{3}\right), 36.77(\mathrm{C}), 30.89\left(\mathrm{CH}_{3}\right) ;{ }^{19} \mathrm{~F} \mathrm{NMR}\left(376 \mathrm{MHz}, \mathrm{CD}_{3} \mathrm{CN}\right) \delta-79.4\left(\mathrm{~s} \mathrm{CF}_{3} \mathrm{SO}_{3}{ }^{-}\right)$; MS (ESI-TOF, positive mode): $m / z 321\left([\mathrm{M}-\mathrm{OTf}]^{+}\right)$; Anal. Calcd for $\mathrm{C}_{18} \mathrm{H}_{15} \mathrm{Cl}_{2} \mathrm{~F}_{3} \mathrm{O}_{3} \mathrm{~S}_{2}$ : C, 45.87; H, 3.21\%; found: C, 46.21. H, 2.95\%.

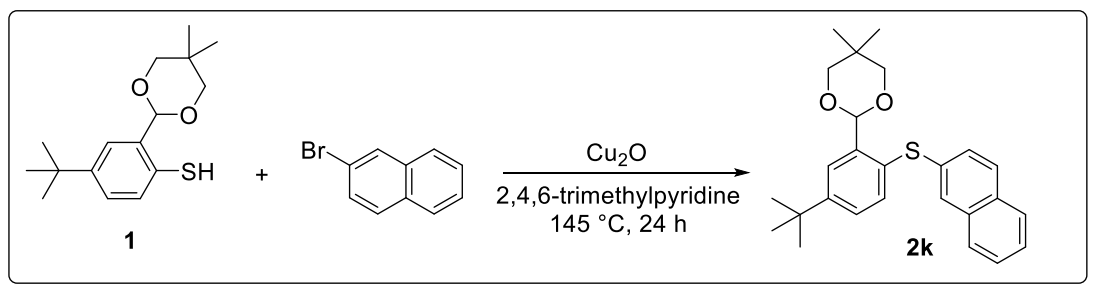

Synthesis of 2-[(5-tert-Butyl)-2-(naphth-2-ylthio)phenyl]-5,5-dimethyl-1,3-dioxane $2 \mathbf{k}$. Benzenethiol 1 (3.001 g, $10.7 \mathrm{mmol}$ ), 2-bromonaphthalene (2.668 g, $12.8 \mathrm{mmol}$ ), and copper(I) oxide $(3.070 \mathrm{~g}, 21.4 \mathrm{mmol})$ were dissolved in 2,4,6-trimethylpyridine $(20 \mathrm{~mL})$. After being stirred at $145^{\circ} \mathrm{C}$ for $24 \mathrm{~h}$ using an oil bath, the reaction mixture was allowed to cool to room temperature. After the solvent was removed under reduced pressure, purification of the crude product by column chromatography on silica gel (eluent: hexane/dichloromethane $\quad=\quad 4 / 1, \quad \mathrm{v} / \mathrm{v}) \quad$ afforded 2-[(5-tert-butyl)-2-(naphth-2-ylthio)phenyl]-5,5-dimethyl-1,3-dioxane $\quad 2 k \quad(1.154 \quad \mathrm{~g}, \quad 2.84$ mmol, 27\%) as pale yellow crystals. 2k: mp 96.8-98.2 ${ }^{\circ} \mathrm{C} ;{ }^{1} \mathrm{H}$ NMR (400 $\left.\mathrm{MHz}, \mathrm{CDCl}_{3}\right) \delta$ $7.83(\mathrm{~d}, J=2.4 \mathrm{~Hz}, 1 \mathrm{H}, \mathrm{ArH}), 7.77-7.73(\mathrm{~m}, 2 \mathrm{H}, \mathrm{ArH}), 7.46-7.38$ (m, 2H, ArH), 7.33-7.27 (m, 1H, ArH), 7.28 (d, J = 2.0 Hz, 1H, ArH), $7.24(\mathrm{~d}, \mathrm{~J}=5.6 \mathrm{~Hz}, 1 \mathrm{H}, \mathrm{ArH}), 5.89$ (s, 1H, CH), $3.73\left(\mathrm{dd}, J=10.8 \mathrm{~Hz}, 2 \mathrm{H}, \mathrm{CH}_{2}\right.$ ), 3.64 (dd, $J=10.8 \mathrm{~Hz}, 2 \mathrm{H}, \mathrm{CH}_{2}$ ), 1.34 (s, 9H, $\mathrm{CH}_{3}$ ), 1.32 (s, $\left.3 \mathrm{H}, \mathrm{CH}_{3}\right), 0.76\left(\mathrm{~s}, 3 \mathrm{H}, \mathrm{CH}_{3}\right) ;{ }^{13} \mathrm{C}\left\{{ }^{1} \mathrm{H}\right\} \mathrm{NMR}\left(101 \mathrm{MHz}, \mathrm{CDCl}_{3}\right) \delta 151.4(\mathrm{C}), 138.9(\mathrm{C}), 134.4$ 
(C), $133.7(\mathrm{C}), 133.2(\mathrm{CH}), 132.0(\mathrm{C}), 129.8(\mathrm{C}), 128.6(\mathrm{CH}), 128.4(\mathrm{CH}), 127.9(\mathrm{CH}), 127.7$ $(\mathrm{CH}), 127.3(\mathrm{CH}), 127.0(\mathrm{CH}), 126.4(\mathrm{CH}), 125.8(\mathrm{CH}), 123.5(\mathrm{CH}), 99.8(\mathrm{CH}), 77.7\left(\mathrm{CH}_{2}\right)$, $34.8(\mathrm{C}), 31.2\left(\mathrm{CH}_{3}\right), 30.2(\mathrm{C}), 23.2\left(\mathrm{CH}_{3}\right), 21.8\left(\mathrm{CH}_{3}\right)$; MS (EI, positive mode): $\mathrm{m} / z 406$ $\left([\mathrm{M}]^{+}\right)$; Anal. Calcd for $\mathrm{C}_{26} \mathrm{H}_{30} \mathrm{O}_{2} \mathrm{~S}: \mathrm{C}, 76.81 ; \mathrm{H}, 7.44 \%$; found: C, 76.95. H, 7.58\%.

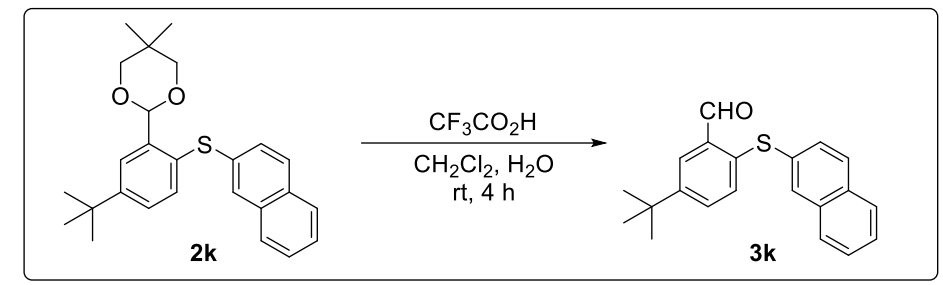

Synthesis of 5-tert-Butyl-2-(naphth-2-ylthio)benzaldehyde 3k. Compound 2k (1.421 g, $3.50 \mathrm{mmol})$ and trifluoroacetic acid $(2.0 \mathrm{~mL})$ was dissolved in a solution of THF $(5 \mathrm{~mL})$ and water $(5 \mathrm{~mL})$. After being stirred at room temperature for $4 \mathrm{~h}$, the solution was extracted with dichloromethane. After the organic layer was washed with water, the organic layers were dried over anhydrous sodium sulfate, filtered, and dried under reduced pressure to give crude pale yellow oil. Purification of the crude oil by column chromatography on silica gel (eluent: hexane/dichloromethane $=1 / 1$, v/v) afforded 5-tert-butyl-2-(naphth-2-ylthio)benzaldehyde 3k (1.119 g, 3.50 mmol, quant.) as pale yellow oil. 3k: ${ }^{1} \mathrm{H}$ NMR (400 MHz, $\left.\mathrm{CDCl}_{3}\right) \delta 10.45$ (s, 1H, CHO), 7.93 (d, $J=2.4 \mathrm{~Hz}, 1 \mathrm{H}, \mathrm{ArH}), 7.90(\mathrm{~d}, J=2.0 \mathrm{~Hz}, 1 \mathrm{H}, \mathrm{ArH}), 7.82(\mathrm{dd}, J=2.4,8.4$ $\mathrm{Hz}, 1 \mathrm{H}, \mathrm{ArH}), 7.78-7.74$ (m, 2H, ArH), 7.52-7.47 (m, 2H, ArH), 7.44 (dd, J=2.4, 8.4 Hz, 1H, $\operatorname{ArH}), 7.32-7.27(\mathrm{~m}, 1 \mathrm{H}, \operatorname{ArH}), 7.13(\mathrm{~d}, J=8.2 \mathrm{~Hz}, 1 \mathrm{H}, \mathrm{ArH}), 1.33\left(\mathrm{~s}, 9 \mathrm{H}, \mathrm{CH}_{3}\right) ;{ }^{13} \mathrm{C}\left\{{ }^{1} \mathrm{H}\right\}$ NMR (101 MHz, $\left.\mathrm{CDCl}_{3}\right) \delta 191.9(\mathrm{C}), 151.4(\mathrm{C}), 150.3(\mathrm{C}), 137.6(\mathrm{C}), 133.9(\mathrm{C}), 133.2(\mathrm{CH})$, $131.7(\mathrm{CH}), 131.2(\mathrm{CH}), 129.4(\mathrm{CH}), 128.2(\mathrm{CH}), 127.8(\mathrm{CH}), 127.6(\mathrm{CH}), 126.8(\mathrm{CH}), 126.7$ (CH), $123.5(\mathrm{C}), 34.6(\mathrm{C}), 31.1\left(\mathrm{CH}_{3}\right)$; MS (EI, positive mode): $\mathrm{m} / z 320\left([\mathrm{M}]^{+}\right)$; HRMS (EI, positive mode): $m / z$ found $406.1975\left([\mathrm{M}]^{+}\right)$, calcd for $\mathrm{C}_{26} \mathrm{H}_{30} \mathrm{O}_{2} \mathrm{~S}: 406.1967$.

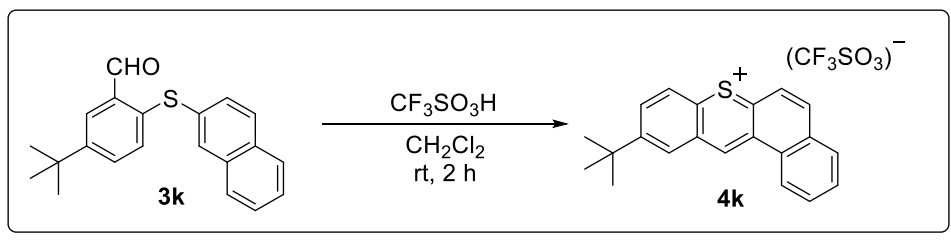




\section{Reaction of 5-tert-Butyl-2-(naphth-2-ylthio)benzaldehyde $3 \mathrm{k}$ with TfOH.}

Trifluoromethanesulfonic acid $(0.100 \mathrm{~g}, 0.666 \mathrm{mmol})$ was added to a solution of compound 3k $(0.100 \mathrm{~g}, 0.312 \mathrm{mmol})$ in dichloromethane $(5 \mathrm{~mL})$ at room temperature. After the solution was stirred at room temperature for $2 \mathrm{~h}$, the reaction mixture was added dropwise to cold $\mathrm{Et}_{2} \mathrm{O}$ $(20 \mathrm{~mL})$. Purification of the resulting suspension using a centrifugal separator afforded exclusively 10-tert-butylbenzo[a]thioxanthen-7-ium trifluoromethanesulfonate $4 \mathbf{k}(0.138 \mathrm{~g}$, 0.306 mmol, 98\%) as red solids. 4k: mp 253.1-254.5 ${ }^{\circ} \mathrm{C} ;{ }^{1} \mathrm{H}$ NMR (400 $\left.\mathrm{MHz}, \mathrm{CD}_{3} \mathrm{CN}\right) \delta$ $10.87(\mathrm{~s}, 1 \mathrm{H}, \operatorname{ArH}), 9.21(\mathrm{~d}, J=8.4 \mathrm{~Hz}, 1 \mathrm{H}, \mathrm{ArH}), 8.97$ (d, $J=2.0 \mathrm{~Hz}, 1 \mathrm{H}, \operatorname{ArH}), 8.74(\mathrm{~d}, J=$ $8.4 \mathrm{~Hz}, 1 \mathrm{H}, \mathrm{ArH}), 8.71(\mathrm{~d}, J=8.0 \mathrm{~Hz}, 1 \mathrm{H}, \mathrm{ArH}), 8.57$ (dd, $J=2.4,9.2 \mathrm{~Hz}, 1 \mathrm{H}, \mathrm{ArH}), 8.53$ (d, $J=6.9 \mathrm{~Hz}, 1 \mathrm{H}, \mathrm{ArH}), 8.35(\mathrm{~d}, J=1.3 \mathrm{~Hz}, 1 \mathrm{H}, \mathrm{ArH}), 8.33(\mathrm{~d}, J=1.3 \mathrm{~Hz}, 1 \mathrm{H}, \operatorname{ArH}), 8.17(\mathrm{dd}, J$ $=7.2,1.4 \mathrm{~Hz}, 1 \mathrm{H}, \mathrm{ArH}), 8.05(\mathrm{dd}, J=7.2,1.4 \mathrm{~Hz}, 1 \mathrm{H}, \mathrm{ArH}), 1.56\left(\mathrm{~s}, 9 \mathrm{H}, \mathrm{CH}_{3}\right) ;{ }^{13} \mathrm{C}\left\{{ }^{1} \mathrm{H}\right\}$ NMR (101 MHz, CD $\left.{ }_{3} \mathrm{CN}\right) \delta 181.5(\mathrm{C}), 156.8(\mathrm{C}), 155.2(\mathrm{C}), 152.6(\mathrm{CH}), 143.6(\mathrm{C}), 142.4$ $(\mathrm{CH}), 137.5(\mathrm{CH}), 133.1(\mathrm{C}), 132.8(\mathrm{C}), 132.5(\mathrm{CH}), 132.0(\mathrm{CH}), 131.79(\mathrm{CH}), 131.77(\mathrm{CH})$, $130.1(\mathrm{C}), 127.9(\mathrm{CH}), 125.0(\mathrm{CH}), 124.8(\mathrm{CH}), 122.0$ (quart, $J_{\mathrm{CF}}=318 \mathrm{~Hz}, \mathrm{CF}_{3} \mathrm{SO}_{3}{ }^{-}$), 36.7 (C), $31.1(\mathrm{CH}) ;{ }^{19} \mathrm{~F}$ NMR $\left(376 \mathrm{MHz}, \mathrm{CD}_{3} \mathrm{CN}\right) \delta-76.4\left(\mathrm{~s}, \mathrm{CF}_{3} \mathrm{SO}_{3}{ }^{-}\right)$; MS (ESI-TOF, positive mode): $m / z 303$ ([M-OTf] $]^{+}$); Anal. Calcd for $\mathrm{C}_{22} \mathrm{H}_{19} \mathrm{~F}_{3} \mathrm{O}_{3} \mathrm{~S}_{2}: \mathrm{C}, 58.40 ; \mathrm{H}, 4.23 \%$; found: $\mathrm{C}$, 58.20. H, $4.15 \%$.

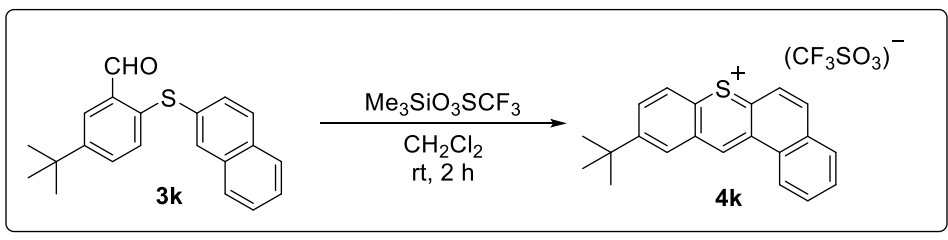

\section{Reaction of 5-tert-Butyl-2-(naphth-2-ylthio)benzaldehyde $3 \mathrm{k}$ with Me3SiOTf.} Trimethylsilyl trifluoromethanesulfonate $(0.167 \mathrm{~g}, 0.751 \mathrm{mmol})$ was added to a solution of compound 3k $(0.102 \mathrm{~g}, 0.318 \mathrm{mmol})$ in dichloromethane $(5 \mathrm{~mL})$ at room temperature. After the solution was stirred at room temperature for $2 \mathrm{~h}$, the reaction mixture was added dropwise to cold $\mathrm{Et}_{2} \mathrm{O}(20 \mathrm{~mL})$. Purification of the resulting suspension using a centrifugal separator afforded exclusively 10-tert-butylbenzo[a]thioxanthen-7-ium trifluoromethanesulfonate $\mathbf{4 k}$ (0.137 g, $0.302 \mathrm{mmol}, 95 \%)$ as red solids. 


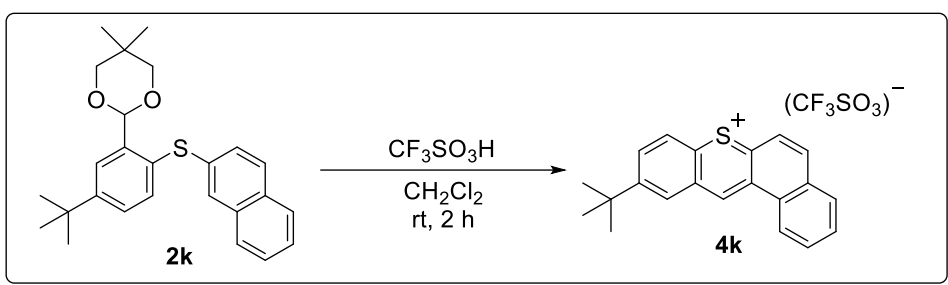

Reaction of $2 \mathbf{k}$ with TfOH. Trifluoromethanesulfonic acid $(0.157 \mathrm{~g}, 1.05 \mathrm{mmol})$ was added to a solution of compound $\mathbf{2 k}(0.202 \mathrm{~g}, 0.500 \mathrm{mmol})$ in dichloromethane $(8 \mathrm{~mL})$ at room temperature. After the solution was stirred at room temperature for $2 \mathrm{~h}$, the reaction mixture was added dropwise to cold $\mathrm{Et}_{2} \mathrm{O}(30 \mathrm{~mL})$. Purification of the resulting suspension using a centrifugal separator afforded exclusively 10-tert-butylbenzo[a]thioxanthen-7-ium trifluoromethanesulfonate $\mathbf{4 k}(0.208 \mathrm{~g}, 0.460 \mathrm{mmol}, 92 \%)$ as red solids.

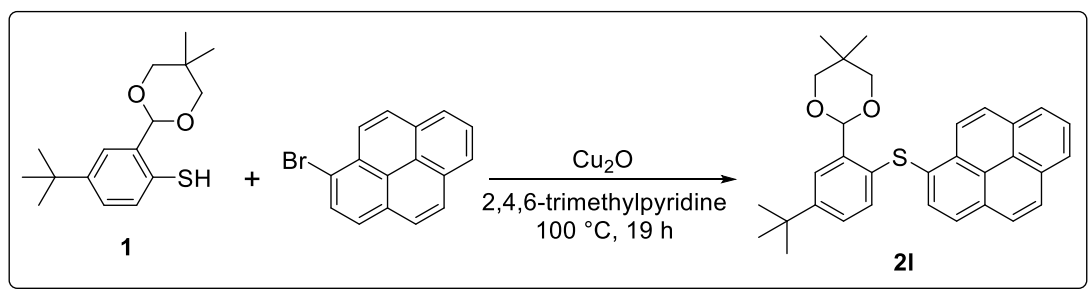

Synthesis of 2-[(5-tert-Butyl)-2-(pyren-1-ylthio)phenyl]-5,5-dimethyl-1,3-dioxane 21 . Benzenethiol 1 (0.847 g, $3.02 \mathrm{mmol})$, 1-bromopyrene (1.018 g, $3.62 \mathrm{mmol})$, and copper(I) oxide $(0.888 \mathrm{~g}, 6.21 \mathrm{mmol})$ were dissolved in 2,4,6-trimethylpyridine $(8 \mathrm{~mL})$. After being stirred at $100{ }^{\circ} \mathrm{C}$ for $19 \mathrm{~h}$ using an oil bath, the reaction mixture was allowed to cool to room temperature. After the solvent was removed under reduced pressure, purification of the crude product by column chromatography on silica gel (eluent: hexane/dichloromethane $=1 / 1$, v/v) afforded 2-[(5-tert-butyl)-2-(pyren-1-ylthio)phenyl]-5,5-dimethyl-1,3-dioxane 21 (0.153 g, $0.318 \mathrm{mmol}, 11 \%$ ) as yellow crystals. $2 \mathrm{l}$ : $\mathrm{mp} 165-167{ }^{\circ} \mathrm{C} ;{ }^{1} \mathrm{H}$ NMR (400 MHz, $\left.\mathrm{CDCl}_{3}\right) \delta 8.66$ (d, $J=9.2 \mathrm{~Hz}, 1 \mathrm{H}, \mathrm{ArH}), 8.20(\mathrm{dd}, J=1.6,7.7 \mathrm{~Hz}, 2 \mathrm{H}, \mathrm{ArH}), 8.13(\mathrm{~d}, J=9.3 \mathrm{~Hz}, 1 \mathrm{H}, \mathrm{ArH})$, 8.09-8.06 (m, 2H, ArH), 8.04-8.00 (m, 2H, ArH), 7.96 (d, J=8.0 Hz, 1H, ArH), 7.82 (d, $J=$ 
$2.3 \mathrm{~Hz}, 1 \mathrm{H}, \mathrm{ArH}), 7.12$ (dd, $J=2.3,8.3 \mathrm{~Hz}, 1 \mathrm{H}, \mathrm{ArH}), 6.89$ (d, $J=8.3 \mathrm{~Hz}, 1 \mathrm{H}, \mathrm{ArH}), 5.99$ (s, 1H, CH), $3.78\left(\mathrm{~d}, J=11.2 \mathrm{~Hz}, 2 \mathrm{H}, \mathrm{CH}_{2}\right), 3.68\left(\mathrm{~d}, J=11.2 \mathrm{~Hz}, 2 \mathrm{H}, \mathrm{CH}_{2}\right), 1.35\left(\mathrm{~s}, 3 \mathrm{H}, \mathrm{CH}_{3}\right)$, $1.29\left(\mathrm{~s}, 3 \mathrm{H}, \mathrm{CH}_{3}\right), 0.77\left(\mathrm{~s}, 3 \mathrm{H}, \mathrm{CH}_{3}\right) ;{ }^{13} \mathrm{C}\left\{{ }^{1} \mathrm{H}\right\} \mathrm{NMR}\left(101 \mathrm{MHz}, \mathrm{CDCl}_{3}\right) \delta 150.3(\mathrm{C}), 137.4$ (C), $131.8(\mathrm{C}), 131.4(\mathrm{C}), 131.3(\mathrm{C}), 131.2(\mathrm{CH}), 131.11(\mathrm{CH}), 131.05(\mathrm{C}), 130.95(\mathrm{C})$, $130.02(\mathrm{C}), 128.3(\mathrm{CH}), 127.7(\mathrm{CH}), 127.3(\mathrm{CH}), 127.0(\mathrm{CH}), 126.2(\mathrm{CH}), 125.4(\mathrm{CH}, 2 \mathrm{C})$, $125.3(\mathrm{C}), 125.2(\mathrm{CH}), 124.7(\mathrm{CH}), 124.5(\mathrm{C}), 123.4(\mathrm{CH}), 99.8(\mathrm{CH}), 77.8\left(\mathrm{CH}_{2}\right), 34.7(\mathrm{C})$, $31.3\left(\mathrm{CH}_{3}\right), 30.3(\mathrm{C}), 23.3\left(\mathrm{CH}_{3}\right), 21.9\left(\mathrm{CH}_{3}\right)$; MS (EI, positive mode): $\mathrm{m} / 2480\left([\mathrm{M}]^{+}\right)$; Anal. Calcd for $\mathrm{C}_{32} \mathrm{H}_{34} \mathrm{O}_{2} \mathrm{~S}$ : C, 79.63; H, 7.10\%; found: C, 79.44; H, 7.07\%.

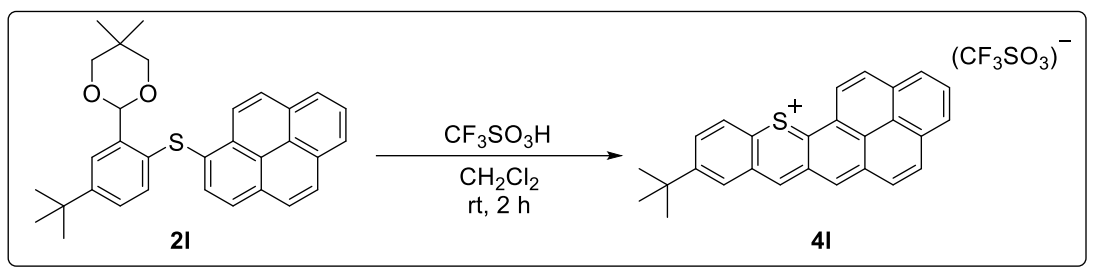

Reaction of 2-[(5-tert-Butyl)-2-(pyren-1-ylthio)phenyl]-5,5-dimethyl-1,3-dioxane 21 with TfOH. Trifluoromethanesulfonic acid $(0.022 \mathrm{~g}, 0.15 \mathrm{mmol})$ was added to a solution of compound $2 \mathbf{i}(0.032 \mathrm{~g}, 0.066 \mathrm{mmol})$ in dichloromethane $(3 \mathrm{~mL})$ at room temperature. After the solution was stirred at room temperature for $2 \mathrm{~h}$, all volatile substances were removed under reduced pressure. The resulting green solid was washed with cold dichloromethane, and dried under reduced pressure to give 9-tert-butylphenaleno[1,9-bc]thioxanthen-12-ium trifluoromethanesulfonate $4 \mathbf{l}(0.030 \mathrm{~g}, 0.057 \mathrm{mmol}, 86 \%)$ as green powder. $4 \mathbf{l}: \mathrm{mp}>300{ }^{\circ} \mathrm{C}$; ${ }^{1} \mathrm{H}$ NMR (400 MHz, CD 3 CN) $\delta 9.31(\mathrm{~s}, 1 \mathrm{H}, \mathrm{ArH}), 8.44(\mathrm{dd}, J=2.0,8.8 \mathrm{~Hz}, 1 \mathrm{H}, \mathrm{ArH}), 8.38$ (brs, 1H, ArH), 8.29 (d, J = 8.8 Hz, 1H, ArH), 8.17 (s, 1H, ArH), 8.06 (d, J = 9.2 Hz, 1H, $\operatorname{ArH}), 8.02(\mathrm{~d}, J=9.2 \mathrm{~Hz}, 1 \mathrm{H}, \operatorname{ArH}), 7.76(\mathrm{~d}, J=7.6 \mathrm{~Hz}, 1 \mathrm{H}, \mathrm{ArH}), 7.70(\mathrm{~d}, J=9.2 \mathrm{~Hz}, 1 \mathrm{H}$, ArH), $7.67(\mathrm{~d}, J=9.2 \mathrm{~Hz}, 1 \mathrm{H}, \operatorname{ArH}), 7.53(\mathrm{~d}, J=6.8 \mathrm{~Hz}, 1 \mathrm{H}, \operatorname{ArH}), 7.46(\mathrm{t}, J=7.6 \mathrm{~Hz}, 1 \mathrm{H}$, ArH); ${ }^{19} \mathrm{~F}$ NMR (376 MHz, CD 3 CN) $\delta-79.4$ (s); MS (ESI-TOF, positive mode): $\mathrm{m} / z 377$ ([M-TfO $]^{+}$); Anal. Calcd for $\mathrm{C}_{28} \mathrm{H}_{21} \mathrm{~F}_{3} \mathrm{O}_{3} \mathrm{~S}_{2}: \mathrm{C}, 63.87 ; \mathrm{H}, 4.02 \%$; found: $\mathrm{C}, 63.57 ; \mathrm{H}, 4.39 \%$. Satisfactory ${ }^{13} \mathrm{C}$ NMR data of $\mathbf{4 l}$ could not be obtained due to its low solubility in common organic solvents. 


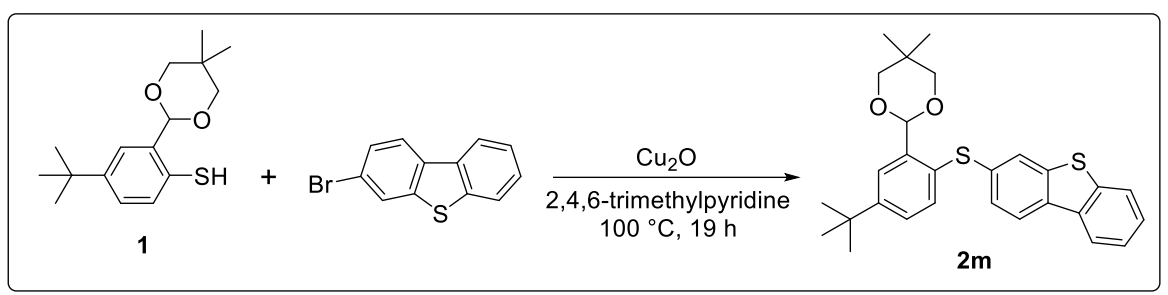

Synthesis of 2m. Benzenethiol 1 (0.729 g, $2.60 \mathrm{mmol}), 3$-bromodibenzo[b,d]thiophene $(0.819$ g, $3.11 \mathrm{mmol})$, and copper(I) oxide $(0.775 \mathrm{~g}, 5.41 \mathrm{mmol})$ were dissolved in 2,4,6-trimethylpyridine (7 mL). After being stirred at $100{ }^{\circ} \mathrm{C}$ for $19 \mathrm{~h}$ using an oil bath, the reaction mixture was allowed to cool to room temperature. After the solvent was removed under reduced pressure, purification of the crude product by column chromatography on silica gel (eluent: dichloromethane/hexane $=1 / 1, \quad \mathrm{v} / \mathrm{v}$ ) afforded 2-[(5-tert-butyl)-2-(dibenzo[b,d]thien-3-ylthio)phenyl]-5,5-dimethyl-1,3-dioxane $2 \mathrm{~m}(0.153 \mathrm{~g}$, $0.331 \mathrm{mmol}, 13 \%)$ as colorless crystals. $2 \mathrm{~m}: \mathrm{mp} 70.5-72.0{ }^{\circ} \mathrm{C} ;{ }^{1} \mathrm{H} \mathrm{NMR}\left(400 \mathrm{MHz}, \mathrm{CDCl}_{3}\right) \delta$ 8.10-8.06 (m, 1H, ArH), 8.01 (dd, $J=0.4,8.0 \mathrm{~Hz}, 1 \mathrm{H}, \mathrm{ArH}), 7.84-7.79$ (m, 2H, ArH), 7.72 (dd, $J=0.5,1.7 \mathrm{~Hz}, 1 \mathrm{H}, \mathrm{ArH}), 7.46-7.41$ (m, 2H, ArH), 7.34-7.31 (m, 2H, ArH), 7.30 (dd, $J$ $=0.7,8.3 \mathrm{~Hz}, 1 \mathrm{H}, \mathrm{ArH}), 5.88(\mathrm{~s}, 1 \mathrm{H}, \mathrm{CH}), 3.73\left(\mathrm{~d}, J=11.2 \mathrm{~Hz}, 2 \mathrm{H}, \mathrm{CH}_{2}\right), 3.64(\mathrm{~d}, J=11.2$ $\left.\mathrm{Hz}, 2 \mathrm{H}, \mathrm{CH}_{2}\right), 1.35$ (s, 9H, $\left.\mathrm{CH}_{3}\right), 1.32\left(\mathrm{~s}, 3 \mathrm{H}, \mathrm{CH}_{3}\right), 0.77$ (s, 3H, $\left.\mathrm{CH}_{3}\right) ;{ }^{13} \mathrm{C}\left\{{ }^{1} \mathrm{H}\right\} \mathrm{NMR}(101$ $\left.\mathrm{MHz}, \mathrm{CDCl}_{3}\right) \delta 151.7$ (C), 140.3 (C), 139.4 (C), 139.2 (C), 136.1 (C), 135.1 (C), 134.0 (C), $133.6(\mathrm{CH}), 129.6(\mathrm{C}), 127.1(\mathrm{CH}), 126.7(\mathrm{CH}), 126.4(\mathrm{CH}), 124.5(\mathrm{CH}), 123.7(\mathrm{CH}), 123.6$ $(\mathrm{CH}), 122.8(\mathrm{CH}), 121.8(\mathrm{CH}), 121.4(\mathrm{CH}), 99.9(\mathrm{CH}), 77.8\left(\mathrm{CH}_{2}\right), 34.8(\mathrm{C}), 31.2\left(\mathrm{CH}_{3}\right), 30.3$ (C), $23.2\left(\mathrm{CH}_{3}\right), 21.8\left(\mathrm{CH}_{3}\right)$; MS (EI, positive mode): $\mathrm{m} / \mathrm{z} 462\left([\mathrm{M}]^{+}\right)$Anal. Calcd for $\mathrm{C}_{28} \mathrm{H}_{30} \mathrm{O}_{2} \mathrm{~S}_{2}$ : C, 72.69; H, 6.54\%; found: C, 72.51; H, 6.88\%.

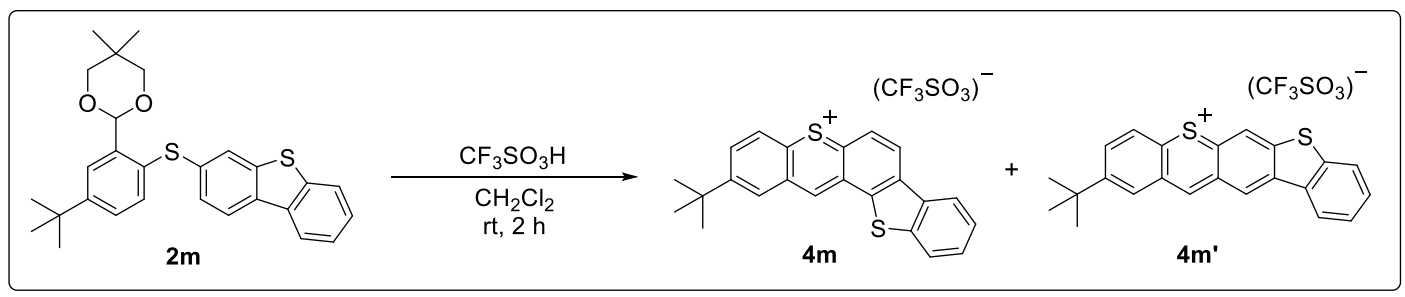


Reaction of $2 \mathrm{~m}$ with TfOH. Trifluoromethanesulfonic acid (0.036 g, $0.24 \mathrm{mmol})$ was added to a solution of compound $\mathbf{2 m}(0.050 \mathrm{~g}, 0.11 \mathrm{mmol})$ in dichloromethane $(5 \mathrm{~mL})$ at room temperature. After the solution was stirred at room temperature for $2 \mathrm{~h}$, all volatile substances were removed under reduced pressure. The resulting green solid was washed with cold dichloromethane, and dried under reduced pressure to give 2-tert-butylbenzo[4,5]thieno[2,3-a]thioxanthen-5-ium trifluoromethanesulfonate $\mathbf{4 m}$ and 2-tert-butylbenzo[4,5]thieno[3,2-b]thioxanthen-5-ium trifluoromethanesulfonate $\mathbf{4 \mathbf { m } ^ { \prime }}$ (0.048 g, $\left.0.094 \mathbf{m m o l}, 85 \%, \mathbf{4 m : 4 m ^ { \prime }}=81: 19\right)$ as reddish brown powder. $\mathbf{4 m}$ and $\mathbf{4 m ^ { \prime }}$ : MS (ESI-TOF, positive mode): $m / z 359\left([\mathrm{M}-\mathrm{OTf}]^{+}\right)$; Anal. Calcd for $\mathrm{C}_{24} \mathrm{H}_{19} \mathrm{~F}_{3} \mathrm{O}_{3} \mathrm{~S}_{3}$ : C, 56.68; $\mathrm{H}, 3.77 \%$; found: C, 56.45; H, 4.08\%. 4m: ${ }^{1} \mathrm{H}$ NMR (400 MHz, $\left.\mathrm{CD}_{3} \mathrm{CN}\right) \delta 10.04$ (s, 1H, ArH), 9.37 (s, 1H, ArH), 9.06 (s, 1H, ArH), $8.72(\mathrm{~d}, J=2.0 \mathrm{~Hz}, 1 \mathrm{H}, \mathrm{ArH}), 8.55$ (d, $J=9.2 \mathrm{~Hz}, 1 \mathrm{H}, \mathrm{ArH})$, $8.50(\mathrm{dd}, J=2.0,9.2 \mathrm{~Hz}, 1 \mathrm{H}, \mathrm{ArH}), 8.42-8.40(\mathrm{~m}, 1 \mathrm{H}, \mathrm{ArH}), 7.91-7.89$ (m, 1H, ArH), 7.62-7.58 (m, 2H, ArH), $1.55\left(\mathrm{~s}, 9 \mathrm{H}, \mathrm{CH}_{3}\right) ;{ }^{13} \mathrm{C}\left\{{ }^{1} \mathrm{H}\right\} \mathrm{NMR}\left(101 \mathrm{MHz}, \mathrm{CD}_{3} \mathrm{CN}\right) \delta 156.2(\mathrm{C})$, $153.0(\mathrm{CH}), 151.2(\mathrm{C}), 148.9(\mathrm{C}), 145.8$ (C), 144.7 (C), 139.7 (C), $138.4(\mathrm{CH}), 136.6(\mathrm{C})$, $135.0(\mathrm{C}), 132.7(\mathrm{CH}), 132.2(\mathrm{CH}), 130.5(\mathrm{C}), 129.8(\mathrm{CH}), 128.0(\mathrm{CH}), 127.2(\mathrm{CH}), 124.4$ $(\mathrm{CH}), 123.7(\mathrm{CH}), 123.6(\mathrm{CH}), 121.7$ (quart, $\left.J_{\mathrm{CF}}=270 \mathrm{~Hz}, \mathrm{CF}_{3}\right) .4 \mathbf{m}^{\prime}:{ }^{1} \mathrm{H}$ NMR (400 MHz, $\left.\mathrm{CD}_{3} \mathrm{CN}\right) \delta 10.18(\mathrm{~s}, 1 \mathrm{H}, \mathrm{ArH}), 8.87(\mathrm{~d}, J=2.0 \mathrm{~Hz}, 1 \mathrm{H}, \mathrm{ArH}), 8.86(\mathrm{~d}, J=12.0 \mathrm{~Hz}, 1 \mathrm{H}, \mathrm{ArH})$, $8.61(\mathrm{~d}, J=8.8 \mathrm{~Hz}, 1 \mathrm{H}, \mathrm{ArH}), 8.56(\mathrm{~d}, J=8.8 \mathrm{~Hz}, 1 \mathrm{H}, \mathrm{ArH}), 8.54(\mathrm{dd}, J=2.0,9.2 \mathrm{~Hz}, 1 \mathrm{H}$, ArH), 8.29 (d, $J=7.6 \mathrm{~Hz}, 1 \mathrm{H}, \mathrm{ArH}), 7.95(\mathrm{~d}, J=7.6 \mathrm{~Hz}, 1 \mathrm{H}, \mathrm{ArH}), 7.54(\mathrm{t}, J=7.6 \mathrm{~Hz}, 1 \mathrm{H}$, ArH), 7.44 (t, $J=7.6 \mathrm{~Hz}, 1 \mathrm{H}, \mathrm{ArH}), 1.55\left(\mathrm{~s}, 9 \mathrm{H}, \mathrm{CH}_{3}\right)$.

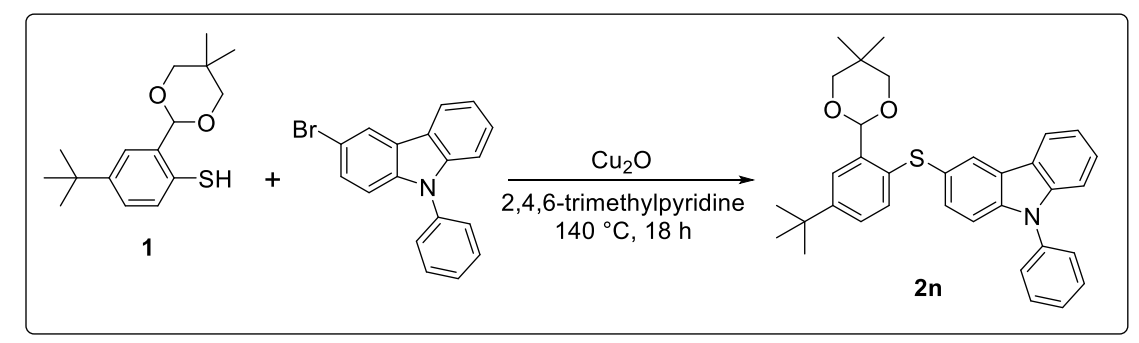

Synthesis

of 3-[(4-tert-Butyl)-2-(5,5-dimethyl-1,3-dioxan-2-yl)phenylthiol)]-9-phenyl-9H-carbazole 2 n. Benzenethiol 1 (0.538 g, $1.92 \mathrm{mmol})$, 3-bromo-9-phenyl-9H-carbazole (0.618 g, $1.92 \mathrm{mmol})$, 
and copper(I) oxide $(0.555 \mathrm{~g}, 3.88 \mathrm{mmol})$ were dissolved in 2,4,6-trimethylpyridine $(8 \mathrm{~mL})$. After being stirred at $140{ }^{\circ} \mathrm{C}$ for $18 \mathrm{~h}$ using an oil bath, the reaction mixture was allowed to cool to room temperature. After the solvent was removed under reduced pressure, purification of the crude product by column chromatography on silica gel (elution with dichloromethane) afforded

3-[(4-tert-butyl)-2-(5,5-dimethyl-1,3-dioxan-2-yl)phenylthiol)]-9-phenyl-9H-carbazole $\quad$ 2n (0.950 g, $1.82 \mathrm{mmol}, 95 \%)$ as pale yellow crystals. $2 \mathrm{n}$ : $\mathrm{mp} 80-82{ }^{\circ} \mathrm{C} ;{ }^{1} \mathrm{H}$ NMR $(400 \mathrm{MHz}$, $\left.\mathrm{CDCl}_{3}\right) \delta 8.27(\mathrm{~d}, J=2.0 \mathrm{~Hz}, 1 \mathrm{H}, \mathrm{ArH}), 7.75(\mathrm{~d}, J=2.0 \mathrm{~Hz}, 1 \mathrm{H}, \mathrm{ArH}), 7.47(\mathrm{dd}, J=2.0,8.4$ $\mathrm{Hz}, 1 \mathrm{H}, \mathrm{ArH}), 7.35$ (d, $J=8.4 \mathrm{~Hz}, 1 \mathrm{H}, \mathrm{ArH}), 7.17$ (dd, $J=2.0,8.4 \mathrm{~Hz}, 1 \mathrm{H}, \operatorname{ArH}), 6.97$ (d, $J=$ $8.4 \mathrm{~Hz}, 1 \mathrm{H}, \mathrm{ArH}), 5.92(\mathrm{~s}, 1 \mathrm{H}, \mathrm{CH}), 3.80\left(\mathrm{~d}, J=10.8 \mathrm{~Hz}, 2 \mathrm{H}, \mathrm{CH}_{2}\right), 3.75(\mathrm{~d}, J=10.8 \mathrm{~Hz}, 2 \mathrm{H}$, $\left.\mathrm{CH}_{2}\right), 1.35$ (s, 3H, CH$), 1.29$ (s, 9H, $\left.\mathrm{CH}_{3}\right), 0.81\left(\mathrm{~s}, 3 \mathrm{H}, \mathrm{CH}_{3}\right) ;{ }^{13} \mathrm{C}\left\{{ }^{1} \mathrm{H}\right\} \mathrm{NMR}(101 \mathrm{MHz}$, $\left.\mathrm{CDCl}_{3}\right) \delta 149.3(\mathrm{C}), 141.3(\mathrm{C}), 140.6(\mathrm{C}), 137.4(\mathrm{C}), 136.1(\mathrm{C}), 133.9(\mathrm{C}), 131.6(\mathrm{CH}), 129.9$ $(\mathrm{CH}), 129.7(\mathrm{CH}), 127.7(\mathrm{CH}), 127.1(\mathrm{CH}), 126.6(\mathrm{CH}), 126.4(\mathrm{CH}), 126.0(\mathrm{CH}), 124.6(\mathrm{C})$, $124.3(\mathrm{C}), 122.9(\mathrm{CH}), 122.8(\mathrm{C}), 120.5(\mathrm{CH}), 120.3(\mathrm{CH}), 110.7(\mathrm{CH}), 109.9(\mathrm{CH}), 99.7$ $(\mathrm{CH}), 77.8\left(\mathrm{CH}_{2}\right), 34.6(\mathrm{C}), 31.3\left(\mathrm{CH}_{3}\right), 30.3(\mathrm{C}), 23.3\left(\mathrm{CH}_{3}\right), 21.9\left(\mathrm{CH}_{3}\right)$; MS (ESI-TOF, positive mode): $m / z 544\left([\mathrm{M}+\mathrm{Na}]^{+}\right)$; Anal. Calcd for $\mathrm{C}_{34} \mathrm{H}_{35} \mathrm{NO}_{2} \mathrm{~S}: \mathrm{C}, 78.27 ; \mathrm{H}, 6.76 ; \mathrm{N}$, 2.68\%; found: C, 77.88; H, 6.65; N, 2.84\%.

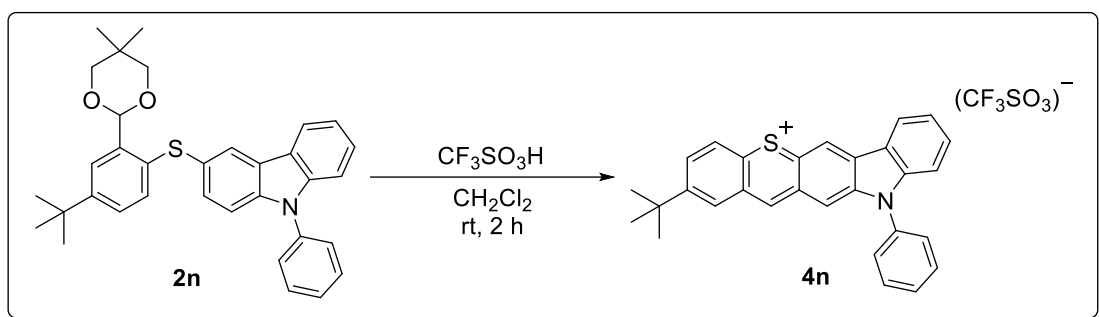

Reaction of 2 n with TfOH. Trifluoromethanesulfonic acid $(0.074 \mathrm{~g}, 0.49 \mathrm{mmol})$ was added to a solution of 3-[(4-tert-butyl)-2-(5,5-dimethyl-1,3-dioxan-2-yl)phenylthiol)]-9-phenyl-9H-carbazole $\mathbf{2 m}$ $(0.100 \mathrm{~g}, 0.192 \mathrm{mmol})$ in dichloromethane $(5 \mathrm{~mL})$ at room temperature. After the solution was stirred at room temperature for $2 \mathrm{~h}$, and was added dropwise to cold $\mathrm{Et}_{2} \mathrm{O}(50 \mathrm{~mL})$. Purification of the resulting suspension using a centrifugal separator afforded 
2-tert-butyl-11-phenyl-11H-thiochromeno[3,2-b]carbazol-5-ium trifluoromethanesulfonate 4n (0.076 g, $0.14 \mathrm{mmol}, 73 \%)$ as dark green powder. 4n: mp 213-215 ${ }^{\circ} \mathrm{C} ;{ }^{1} \mathrm{H}$ NMR $(400 \mathrm{MHz}$, $\left.\mathrm{CD}_{3} \mathrm{CN}\right) \delta 9.99(\mathrm{~s}, 1 \mathrm{H}, \mathrm{ArH}), 9.42(\mathrm{~s}, 1 \mathrm{H}, \mathrm{ArH}), 8.62(\mathrm{~s}, 1 \mathrm{H}, \mathrm{ArH}), 8.59(\mathrm{~d}, J=9.2 \mathrm{~Hz}, 1 \mathrm{H}$, ArH), 8.59 (d, $J=2.0 \mathrm{~Hz}, 1 \mathrm{H}, \mathrm{ArH}), 8.47$ (d, $J=7.6 \mathrm{~Hz}, 1 \mathrm{H}, \mathrm{ArH}), 8.45$ (dd, $J=2.0,9.2 \mathrm{~Hz}$, 1H, ArH), 7.83-7.70 (m, 6H, ArH), 7.50-7.44 (m, 2H, ArH), $1.51\left(\mathrm{~s}, 9 \mathrm{H}, \mathrm{CH}_{3}\right) ;{ }^{13} \mathrm{C}\left\{{ }^{1} \mathrm{H}\right\}$ NMR (101 MHz, CD $\left.{ }_{3} \mathrm{CN}\right) \delta 159.8(\mathrm{CH}), 154.6(\mathrm{C}), 147.7$ (C), 145.9 (C), $143.4(\mathrm{C}), 139.1$ (C), $137.9(\mathrm{CH}), 136.6(\mathrm{C}), 136.0(\mathrm{C}), 134.5(\mathrm{CH}), 132.5(\mathrm{CH}), 131.7(\mathrm{CH} \times 2), 130.3(\mathrm{CH})$, $130.2(\mathrm{C}), 129.2(\mathrm{C}), 128.3(\mathrm{CH}), 127.7(\mathrm{CH} \times 2), 125.0(\mathrm{CH}), 123.4(\mathrm{CH}), 119.6$ (quart, $J_{\mathrm{CF}}=$ $\left.320 \mathrm{~Hz}, \mathrm{CF}_{3}\right), 119.0(\mathrm{C}), 118.9(\mathrm{CH}), 116.0(\mathrm{CH}), 112.0(\mathrm{CH}), 36.4(\mathrm{C}), 31.0\left(\mathrm{CH}_{3}\right) ;{ }^{19} \mathrm{~F}$ NMR (376 MHz, CD $\left.{ }_{3} \mathrm{CN}\right) \delta-79.4$ (s); MS (ESI-TOF, positive mode): $m / z 418$ ([M-OTf] ${ }^{+}$); Anal. Calcd for $\mathrm{C}_{30} \mathrm{H}_{24} \mathrm{~F}_{3} \mathrm{NO}_{3} \mathrm{~S}_{2}$ : C, 63.48; H, 4.26; N, 2.47\%; found: C, 63.23; H, 4.49; N, 2.43\%. 


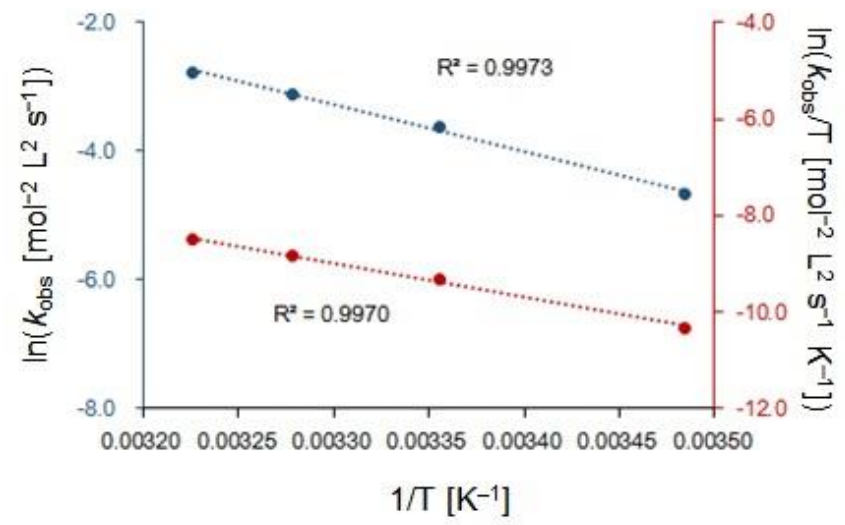

Figure S1. Arrhenius (blue) and Eyring-Polanyi (red) plots for the TfOH-promoted intramolecular cyclization of thioether $\mathbf{3 b}$. 


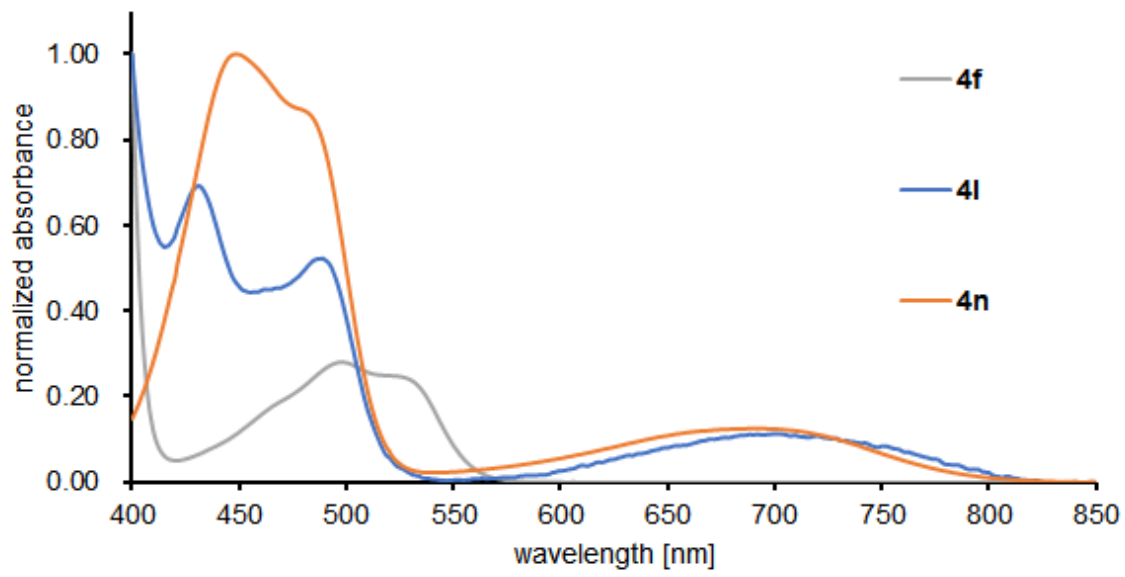

Figure S2. UV-vis absorption spectra of thiopyrylium trifluoromethanesulfonates $\mathbf{4 f}, \mathbf{4 l}$, and $4 n$ in $\mathrm{CH}_{2} \mathrm{Cl}_{2}$ at room temperature.

Table S1. Photophysical properties of $\mathbf{4 f}, \mathbf{4 l}$, and $\mathbf{4 n}$ in $\mathrm{CH}_{2} \mathrm{Cl}_{2}$ at room temperature.

\begin{tabular}{lll}
\hline Compd. & \multicolumn{2}{l}{ Absorption } \\
\cline { 2 - 3 } & $\lambda_{\max }[\mathrm{nm}]$ & $\varepsilon\left[\mathrm{cm}^{-1} \mathrm{M}^{-1}\right]$ \\
\hline 4f & 533 & 2200 \\
4l & 701 & 889 \\
4n & 693 & 2530 \\
\hline
\end{tabular}


Theoretical Calculations. All density functional theory (DFT) calculations were performed utilizing the Gaussian 16 package. ${ }^{2}$ The Becke's three-parameter hybrid functional with Lee-Yang-Parr correlation functional (B3LYP) ${ }^{3}$ and Truhlar group's new functional (MN15), ${ }^{4}$ which was recently reported to demonstrate broader accuracy, were employed with a standard split valence-type basis sets. The geometries of $\mathbf{4 f}^{\mathbf{\prime}}, \mathbf{4} \mathbf{l}^{\prime}$, and $\mathbf{4 n}^{\prime}$, where the counter anions were omitted, were optimized by the $6-311+\mathrm{G}(2 \mathrm{~d}, \mathrm{p})$ basis sets. Harmonic vibrational frequency calculations were carried out for the stationary points to confirm each structure being either a minimum, with no imaginary frequency, or a transition structure (TS), with one imaginary frequency. Molecular orbitals and TD-DFT calculations were carried out at the $6-311+\mathrm{G}(2 \mathrm{~d}, \mathrm{p})$ levels using the B3LYP density functional models. Moreover, reaction paths including geometry optimization of transition states (TSs) were calculated using the synchronous transit-guided quasi-Newton method. ${ }^{5}$ The nature of stationary points obtained by the geometry optimization, that is yielding one imaginary frequency for TSs and none for the minima, was checked by the harmonic vibrational analysis at the same calculation level. Each transition state was further confirmed by intrinsic reaction coordinate (IRC) approach ${ }^{6}$ where the minima on the energy profile was obtained by following its normal mode downhill from TS on both reaction sides. The Gibbs free energies of minima and TSs were estimated with zero-point energy (ZPE) and thermal energy corrections obtained in the condition of 298.15 K and $1 \mathrm{~atm}$. The bulk solvent effects of dichloromethane might be adequately evaluated by using polarizable continuum model (PCM) ${ }^{7}$, denoted as " $\mathrm{PCM}\left(\mathrm{CH}_{2} \mathrm{Cl}_{2}\right)$-".

The relative Gibbs free energies in the reaction path was based on the protonated thioether solvated by a $\mathrm{TfOH}$ molecule $(\mathbf{3 c})$. Though the protonation step of neutral thioether was significantly unfavorable, where the free energies were calculated to be about $20 \mathrm{kcal} / \mathrm{mol}$ at both functionals, an excess of $\mathrm{TfOH}$ in our experimental condition shifted the equilibrium of the reaction toward the unstable side, implying that the protonated one might be a reasonable starting point in the energy profile. The desolvation of a $\mathrm{TfOH}$ molecule and subsequent resolvation process ( $\mathbf{B} \rightarrow \mathbf{C} \rightarrow \mathbf{D}$ in Figure 1) might include sufficiently large basis-set superposition-error (BSSE) due to our lower level of a basis set, so that a BSSE correction using a counter-poise method ${ }^{8}$ was applied to obtain more accurate binding energies. Here, it should be noted that the reorientation of $\mathrm{TfOH}$ which directly proceeds from $\mathbf{B}$ to $\mathbf{D}$ with 
maintaining hydrogen bonds was calculated to be much less stable. On the other hand, the desolvated intermediate acquires stability driven by the entropy increase.

All computations were carried out using the computer facilities at Research Institute for Information Technology, Kyushu University.

Table S2. Summary of the computational results.

\begin{tabular}{lllll}
\hline Compound & $\begin{array}{l}\text { Point } \\
\text { group }\end{array}$ & $\begin{array}{l}\text { Dipole } \\
\text { moment } \\
\text { (Debye) }\end{array}$ & $\begin{array}{l}\text { Number of imaginary } \\
\text { frequencies }\end{array}$ & $\begin{array}{l}\text { Computed total } \\
\text { energies (Hartree) }\end{array}$ \\
\hline $\mathbf{4 f}^{\prime}$ & $C \mathrm{~s}$ & 3.354313 & 0 & -1095.564099 \\
$\mathbf{4 l ^ { \prime }}$ & $C \mathrm{~s}$ & 0.845149 & 0 & -1439.854481 \\
$\mathbf{4 n}^{\prime}$ & $C \mathrm{~s}$ & 1.581817 & 0 & -1572.652837 \\
\hline
\end{tabular}



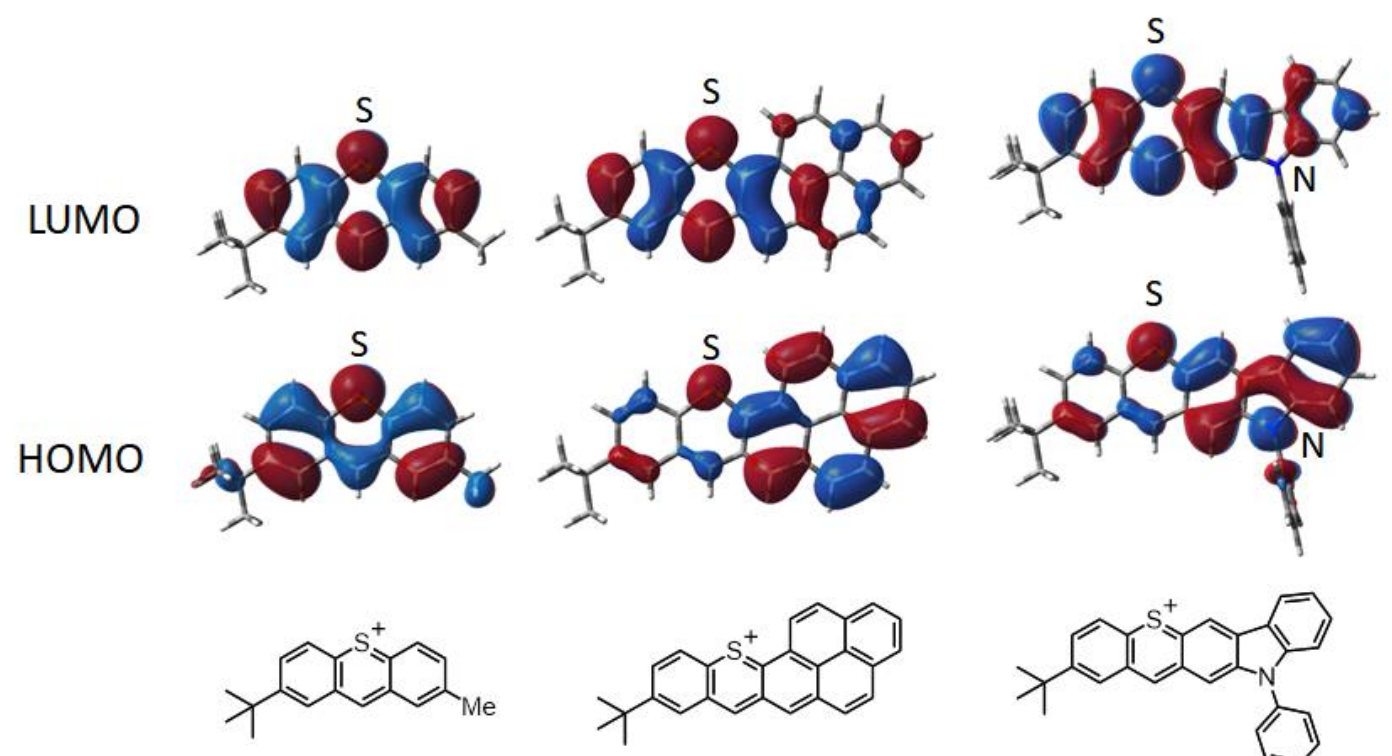

4f'

$4 I^{\circ}$

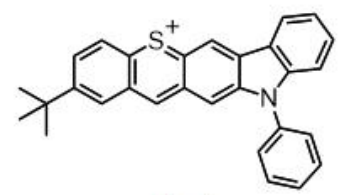

$4 n^{\prime}$

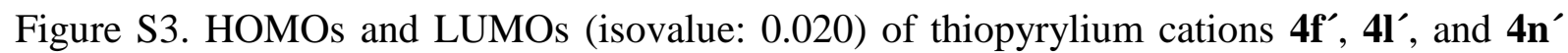
calculated at the B3LYP/6-311+G(2d,p) level of theory.

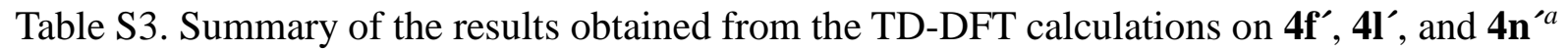

\begin{tabular}{llllll}
\hline Compound & $E[\mathrm{eV}]^{b}$ & $\lambda[\mathrm{nm}]^{c}$ & $f^{d}$ & Transition \\
\hline $\mathbf{4 f}^{\prime}$ & 2.515 & 493.0 & 0.0450 & HOMO $\rightarrow$ LUMO \\
$\mathbf{4 l}^{\prime}$ & 1.657 & 748.1 & 0.0242 & HOMO $\rightarrow$ LUMO & \\
$\mathbf{4 n}^{\prime}$ & 1.825 & 679.3 & 0.0345 & HOMO $\rightarrow$ LUMO & \\
\hline \multicolumn{2}{l}{ Calculations } & were & \multicolumn{2}{c}{ carried } & out
\end{tabular}

$\operatorname{PCM}\left(\mathrm{CH}_{2} \mathrm{Cl}_{2}\right)-\mathrm{B} 3 \mathrm{LYP} / 6-311+\mathrm{G}(2 \mathrm{~d}, \mathrm{p}) / / \mathrm{B} 3 \mathrm{LYP} / 6-311+\mathrm{G}(2 \mathrm{~d}, \mathrm{p})$ level of theory.

${ }^{b}$ Excitation energy.

${ }^{c}$ Excitation wavelength.

${ }^{d}$ Oscillator strength. 
Table S4. Theoretically optimized cartesian coordinates of thiopyrylium cation $\mathbf{4 f}^{\prime}$ at B3LYP/6-311+G(2d,p) level of theory.

\begin{tabular}{llll}
\hline atom & $\mathrm{x}$ & $\mathrm{y}$ & $\mathrm{z}$ \\
\hline $\mathrm{C}$ & 1.235228 & 0.321244 & 0 \\
$\mathrm{C}$ & -0.1906 & 0.340355 & 0 \\
$\mathrm{C}$ & -0.93755 & -0.84043 & 0 \\
$\mathrm{C}$ & -0.42828 & -2.14536 & 0 \\
$\mathrm{C}$ & 0.97562 & -2.40036 & 0 \\
$\mathrm{~S}$ & 2.153105 & -1.13795 & 0 \\
$\mathrm{C}$ & 1.938149 & 1.533299 & 0 \\
$\mathrm{C}$ & 1.24791 & 2.72236 & 0 \\
$\mathrm{C}$ & -0.17347 & 2.794072 & 0 \\
$\mathrm{C}$ & -0.85509 & 1.602017 & 0 \\
$\mathrm{C}$ & -1.3157 & -3.25536 & 0 \\
$\mathrm{C}$ & -0.86228 & -4.55359 & 0 \\
$\mathrm{C}$ & 0.538866 & -4.76222 & 0 \\
$\mathrm{C}$ & 1.440648 & -3.72113 & 0 \\
$\mathrm{C}$ & -0.85987 & 4.163254 & 0 \\
$\mathrm{C}$ & -2.39148 & 4.040774 & 0 \\
$\mathrm{C}$ & -0.42828 & 4.942455 & 1.263494 \\
$\mathrm{C}$ & -0.42828 & 4.942455 & -1.26349 \\
$\mathrm{C}$ & -1.81481 & -5.7195 & 0 \\
$\mathrm{H}$ & -2.018 & -0.73719 & 0 \\
$\mathrm{H}$ & 3.021366 & 1.533782 & 0 \\
$\mathrm{H}$ & 1.821949 & 3.639978 & 0 \\
$\mathrm{H}$ & -1.93636 & 1.586322 & 0 \\
$\mathrm{H}$ & -2.38101 & -3.05249 & 0 \\
$\mathrm{H}$ & 0.916559 & -5.77802 & 0 \\
$\mathrm{H}$ & 2.50472 & -3.92357 & 0 \\
$\mathrm{H}$ & -2.83436 & 5.037602 & 0 \\
$\mathrm{H}$ & -2.76194 & 3.5225 & -0.88781 \\
$\mathrm{H}$ & -2.76194 & 3.5225 & 0.887813 \\
$\mathrm{H}$ & -0.90646 & 5.923728 & 1.271395 \\
$\mathrm{H}$ & 0.650394 & 5.105543 & 1.302108 \\
$\mathrm{H}$ & -0.72414 & 4.416095 & 2.173607 \\
$\mathrm{H}$ & -0.72414 & 4.416095 & -2.17361 \\
$\mathrm{H}$ & -0.90646 & 5.923728 & -1.2714 \\
$\mathrm{H}$ & 0.650394 & 5.105543 & -1.30211 \\
$\mathrm{H}$ & -1.28244 & -6.66988 & 0 \\
$\mathrm{H}$ & -2.46103 & -5.69556 & 0.880323 \\
$\mathrm{H}$ & -2.46103 & -5.69556 & -0.88032 \\
\hline & & & \\
\hline
\end{tabular}


Table S5. Theoretically optimized cartesian coordinates of thiopyrylium cation $4 \mathbf{I}^{\prime}$ at B3LYP/6-311+G(2d,p) level of theory.

\begin{tabular}{|c|c|c|c|}
\hline atom & $\mathrm{x}$ & $\mathrm{y}$ & $\mathrm{z}$ \\
\hline $\mathrm{C}$ & -1.05481 & -2.16609 & 0 \\
\hline C & 0.352475 & -2.3631 & 0 \\
\hline $\mathrm{C}$ & 1.232844 & -1.27751 & 0 \\
\hline C & 0.89157 & 0.079387 & 0 \\
\hline C & -0.46968 & 0.52408 & 0 \\
\hline$S$ & -1.78478 & -0.60407 & 0 \\
\hline $\mathrm{C}$ & -1.90499 & -3.2799 & 0 \\
\hline C & -1.36934 & -4.54646 & 0 \\
\hline C & 0.031292 & -4.79431 & 0 \\
\hline C & 0.856427 & -3.69595 & 0 \\
\hline C & 1.928418 & 1.045062 & 0 \\
\hline C & 1.663637 & 2.396279 & 0 \\
\hline C & 0.294639 & 2.842676 & 0 \\
\hline $\mathrm{C}$ & -0.77742 & 1.907408 & 0 \\
\hline C & 0.54277 & -6.23858 & 0 \\
\hline C & 2.077797 & -6.3072 & 0 \\
\hline C & 0.018996 & -6.95922 & 1.263164 \\
\hline C & 0.018996 & -6.95922 & -1.26316 \\
\hline C & 2.718562 & 3.373544 & 0 \\
\hline C & 2.443038 & 4.696239 & 0 \\
\hline $\mathrm{C}$ & 1.088636 & 5.177862 & 0 \\
\hline C & 0.018996 & 4.235199 & 0 \\
\hline C & -1.32681 & 4.69855 & 0 \\
\hline C & -2.38005 & 3.735702 & 0 \\
\hline C & -2.12054 & 2.403403 & 0 \\
\hline C & 0.791307 & 6.539651 & 0 \\
\hline $\mathrm{C}$ & -0.5305 & 6.983417 & 0 \\
\hline C & -1.57842 & 6.078194 & 0 \\
\hline $\mathrm{H}$ & 2.293175 & -1.50893 & 0 \\
\hline $\mathrm{H}$ & -2.97965 & -3.14413 & 0 \\
\hline $\mathrm{H}$ & -2.05382 & -5.38488 & 0 \\
\hline $\mathrm{H}$ & 1.931377 & -3.81414 & 0 \\
\hline $\mathrm{H}$ & 2.954485 & 0.697119 & 0 \\
\hline $\mathrm{H}$ & 2.393827 & -7.3513 & 0 \\
\hline $\mathrm{H}$ & 2.509374 & -5.83828 & -0.88761 \\
\hline $\mathrm{H}$ & 2.509374 & -5.83828 & 0.887612 \\
\hline $\mathrm{H}$ & 0.372628 & -7.99206 & 1.271718 \\
\hline $\mathrm{H}$ & -1.07157 & -6.98759 & 1.301718 \\
\hline $\mathrm{H}$ & 0.377201 & -6.47263 & 2.17305 \\
\hline
\end{tabular}




\begin{tabular}{llll}
\hline $\mathrm{H}$ & 0.377201 & -6.47263 & -2.17305 \\
$\mathrm{H}$ & 0.372628 & -7.99206 & -1.27172 \\
$\mathrm{H}$ & -1.07157 & -6.98759 & -1.30172 \\
$\mathrm{H}$ & 3.743473 & 3.022853 & 0 \\
$\mathrm{H}$ & 3.247154 & 5.422598 & 0 \\
$\mathrm{H}$ & -3.40513 & 4.086651 & 0 \\
$\mathrm{H}$ & -2.95166 & 1.709982 & 0 \\
$\mathrm{H}$ & 1.601607 & 7.258957 & 0 \\
$\mathrm{H}$ & -0.73772 & 8.046262 & 0 \\
$\mathrm{H}$ & -2.603 & 6.43024 & 0 \\
\hline
\end{tabular}

Table S6. Theoretically optimized cartesian coordinates of thiopyrylium cation $4 \mathbf{n}^{\prime}$ at B3LYP/6-311+G(2d,p) level of theory.

\begin{tabular}{llll}
\hline atom & $\mathrm{x}$ & $\mathrm{y}$ & $\mathrm{z}$ \\
\hline $\mathrm{C}$ & 2.508705 & -2.2314 & 0 \\
$\mathrm{C}$ & 2.693216 & -0.8228 & 0 \\
$\mathrm{C}$ & 1.604078 & 0.06153 & 0 \\
$\mathrm{C}$ & 0.251931 & -0.27773 & 0 \\
$\mathrm{C}$ & -0.1795 & -1.65438 & 0 \\
$\mathrm{~S}$ & 0.949231 & -2.97274 & 0 \\
$\mathrm{C}$ & 3.630344 & -3.07147 & 0 \\
$\mathrm{C}$ & 4.893338 & -2.52747 & 0 \\
$\mathrm{C}$ & 5.128546 & -1.12608 & 0 \\
$\mathrm{C}$ & 4.022115 & -0.31068 & 0 \\
$\mathrm{C}$ & -0.72615 & 0.755276 & 0 \\
$\mathrm{C}$ & -2.05152 & 0.413369 & 0 \\
$\mathrm{C}$ & -2.4748 & -0.96588 & 0 \\
$\mathrm{C}$ & -1.53125 & -1.98293 & 0 \\
$\mathrm{C}$ & 6.568257 & -0.60055 & 0 \\
$\mathrm{C}$ & 6.622792 & 0.935104 & 0 \\
$\mathrm{C}$ & 7.294697 & -1.11664 & 1.262823 \\
$\mathrm{C}$ & 7.294697 & -1.11664 & -1.26282 \\
$\mathrm{~N}$ & -3.17006 & 1.219974 & 0 \\
$\mathrm{C}$ & -4.3015 & 0.406251 & 0 \\
$\mathrm{C}$ & -3.90549 & -0.9548 & 0 \\
$\mathrm{C}$ & -5.64246 & 0.775554 & 0 \\
$\mathrm{C}$ & -6.58217 & -0.24492 & 0 \\
$\mathrm{C}$ & -6.20999 & -1.6012 & 0 \\
$\mathrm{C}$ & -4.87768 & -1.96459 & 0 \\
$\mathrm{C}$ & -3.16627 & 2.654548 & 0 \\
$\mathrm{C}$ & -3.16743 & 3.343302 & 1.209882 \\
\hline & & &
\end{tabular}




\begin{tabular}{llll}
\hline $\mathrm{C}$ & -3.16743 & 4.734071 & 1.206018 \\
$\mathrm{C}$ & -3.16708 & 5.428295 & 0 \\
$\mathrm{C}$ & -3.16743 & 4.734071 & -1.20602 \\
$\mathrm{C}$ & -3.16743 & 3.343302 & -1.20988 \\
$\mathrm{H}$ & 1.839951 & 1.120933 & 0 \\
$\mathrm{H}$ & 3.502583 & -4.14718 & 0 \\
$\mathrm{H}$ & 5.736728 & -3.20584 & 0 \\
$\mathrm{H}$ & 4.132269 & 0.765188 & 0 \\
$\mathrm{H}$ & -0.40763 & 1.789765 & 0 \\
$\mathrm{H}$ & -1.84054 & -3.0212 & 0 \\
$\mathrm{H}$ & 7.663951 & 1.260999 & 0 \\
$\mathrm{H}$ & 6.149467 & 1.362104 & -0.8875 \\
$\mathrm{H}$ & 6.149467 & 1.362104 & 0.887497 \\
$\mathrm{H}$ & 8.324295 & -0.75335 & 1.271524 \\
$\mathrm{H}$ & 7.332797 & -2.20686 & 1.301527 \\
$\mathrm{H}$ & 6.804487 & -0.76317 & 2.172652 \\
$\mathrm{H}$ & 6.804487 & -0.76317 & -2.17265 \\
$\mathrm{H}$ & 8.324295 & -0.75335 & -1.27152 \\
$\mathrm{H}$ & 7.332797 & -2.20686 & -1.30153 \\
$\mathrm{H}$ & -5.94163 & 1.815217 & 0 \\
$\mathrm{H}$ & -7.6346 & 0.011069 & 0 \\
$\mathrm{H}$ & -6.9788 & -2.36268 & 0 \\
$\mathrm{H}$ & -4.59209 & -3.00945 & 0 \\
$\mathrm{H}$ & -3.17426 & 2.791737 & 2.142165 \\
$\mathrm{H}$ & -3.17182 & 5.273913 & 2.144702 \\
$\mathrm{H}$ & -3.17031 & 6.511242 & 0 \\
$\mathrm{H}$ & -3.17182 & 5.273913 & -2.1447 \\
$\mathrm{H}$ & -3.17426 & 2.791737 & -2.14217 \\
\hline & & &
\end{tabular}

Table S7. Theoretically optimized cartesian coordinates of intermediates and transition states in the reaction pathway for the cyclization of thioether $\mathbf{3 c}$ at the $\operatorname{PCM}\left(\mathrm{CH}_{2} \mathrm{Cl}_{2}\right)$-B3LYP/6-31G(d) level.

\begin{tabular}{lrrr} 
TS1 & \multicolumn{3}{l}{} \\
\hline atom & $\mathrm{x}$ & $\mathrm{y}$ & $\mathrm{z}$ \\
\hline $\mathrm{C}$ & -1.94812 & 1.880798 & -0.33608 \\
$\mathrm{C}$ & -1.14302 & 2.90331 & -0.84892 \\
$\mathrm{C}$ & -1.71086 & 1.375887 & 0.98813 \\
$\mathrm{C}$ & -0.12942 & 3.441739 & -0.06323 \\
$\mathrm{C}$ & -0.66782 & 1.980467 & 1.767478 \\
$\mathrm{C}$ & 0.125943 & 2.973917 & 1.24583 \\
$\mathrm{C}$ & -3.49448 & -0.40118 & -0.57089 \\
$\mathrm{C}$ & -2.55403 & -1.07008 & 0.238536 \\
$\mathrm{C}$ & -4.74804 & -0.95947 & -0.80179
\end{tabular}




\begin{tabular}{lrrr}
$\mathrm{C}$ & -2.90183 & -2.30046 & 0.803547 \\
$\mathrm{C}$ & -5.07486 & -2.2023 & -0.24335 \\
$\mathrm{C}$ & -4.15507 & -2.87043 & 0.557424 \\
$\mathrm{C}$ & -1.20238 & -0.50125 & 0.51356 \\
$\mathrm{C}$ & 4.092501 & -1.17719 & -0.59557 \\
$\mathrm{~F}$ & 4.662374 & -0.60594 & -1.64604 \\
$\mathrm{~F}$ & 4.99058 & -1.39441 & 0.358448 \\
$\mathrm{~F}$ & 3.506558 & -2.31433 & -0.94627 \\
$\mathrm{H}$ & -1.29133 & 3.259515 & -1.8632 \\
$\mathrm{H}$ & -2.56038 & 1.001861 & 1.552893 \\
$\mathrm{H}$ & 0.493997 & 4.227737 & -0.47879 \\
$\mathrm{H}$ & -0.51671 & 1.622901 & 2.781573 \\
$\mathrm{H}$ & 0.927706 & 3.412175 & 1.829844 \\
$\mathrm{H}$ & -5.47158 & -0.43152 & -1.41504 \\
$\mathrm{H}$ & -2.17985 & -2.81364 & 1.428053 \\
$\mathrm{H}$ & -6.05409 & -2.63083 & -0.43207 \\
$\mathrm{H}$ & -4.40565 & -3.82768 & 1.002944 \\
$\mathrm{H}$ & -0.57317 & -0.25098 & -0.34136 \\
$\mathrm{H}$ & 0.392863 & -0.90446 & 1.492966 \\
$\mathrm{H}$ & 3.945896 & 1.079513 & 1.505764 \\
$\mathrm{O}$ & -0.58057 & -1.05739 & 1.545727 \\
$\mathrm{O}$ & 2.163068 & -0.71286 & 1.229618 \\
$\mathrm{O}$ & 1.950667 & 0.434241 & -1.03022 \\
$\mathrm{O}$ & 3.649953 & 1.214188 & 0.580269 \\
$\mathrm{~S}$ & -3.11084 & 1.133354 & -1.41915 \\
$\mathrm{~S}$ & 2.76463 & -0.03213 & 0.076029 \\
\hline & & &
\end{tabular}

\begin{tabular}{lrrr}
\multicolumn{4}{l}{ Intermediate B } \\
\hline atom & $\mathrm{X}$ & $\mathrm{y}$ & \multicolumn{2}{l}{$\mathrm{z}$} \\
\hline $\mathrm{C}$ & -2.73396 & 1.455101 & -0.51952 \\
$\mathrm{C}$ & -2.86831 & 2.747778 & -1.0461 \\
$\mathrm{C}$ & -2.02756 & 1.230977 & 0.777955 \\
$\mathrm{C}$ & -2.29175 & 3.821592 & -0.39125 \\
$\mathrm{C}$ & -1.37753 & 2.440783 & 1.352505 \\
$\mathrm{C}$ & -1.53358 & 3.673761 & 0.80961 \\
$\mathrm{C}$ & -2.96225 & -1.28311 & -0.44785 \\
$\mathrm{C}$ & -1.90275 & -1.27957 & 0.473762 \\
$\mathrm{C}$ & -3.70301 & -2.4411 & -0.71073 \\
$\mathrm{C}$ & -1.60193 & -2.47559 & 1.127442 \\
$\mathrm{C}$ & -3.38272 & -3.62363 & -0.04608 \\
$\mathrm{C}$ & -2.33301 & -3.63857 & 0.87278 \\
$\mathrm{C}$ & -1.08147 & -0.02519 & 0.746729 \\
$\mathrm{C}$ & 4.008138 & -0.8829 & -0.60809 \\
$\mathrm{~F}$ & 4.599169 & -0.47996 & -1.72428 \\
$\mathrm{~F}$ & 4.917184 & -1.2037 & 0.30578 \\
$\mathrm{~F}$ & 3.217958 & -1.92148 & -0.8538 \\
\hline
\end{tabular}




\begin{tabular}{lrrr}
\hline $\mathrm{H}$ & -3.40612 & 2.903427 & -1.97563 \\
$\mathrm{H}$ & -2.81528 & 0.956856 & 1.51132 \\
$\mathrm{H}$ & -2.40459 & 4.812514 & -0.82136 \\
$\mathrm{H}$ & -0.80057 & 2.283322 & 2.25682 \\
$\mathrm{H}$ & -1.07932 & 4.547703 & 1.263081 \\
$\mathrm{H}$ & -4.52022 & -2.41705 & -1.42556 \\
$\mathrm{H}$ & -0.79451 & -2.47848 & 1.8503 \\
$\mathrm{H}$ & -3.95575 & -4.52378 & -0.24444 \\
$\mathrm{H}$ & -2.08386 & -4.55424 & 1.400251 \\
$\mathrm{H}$ & -0.35934 & 0.127383 & -0.06744 \\
$\mathrm{H}$ & 0.537437 & -0.01609 & 1.834417 \\
$\mathrm{H}$ & 4.397069 & 1.58086 & 1.234798 \\
$\mathrm{O}$ & -0.42028 & -0.12643 & 1.983674 \\
$\mathrm{O}$ & 2.379741 & 0.046963 & 1.330892 \\
$\mathrm{O}$ & 2.086605 & 0.974774 & -1.02092 \\
$\mathrm{O}$ & 4.04392 & 1.647637 & 0.322307 \\
$\mathrm{~S}$ & -3.3935 & 0.154051 & -1.40948 \\
$\mathrm{~S}$ & 2.937667 & 0.505833 & 0.056944 \\
\hline
\end{tabular}

Intermediate D

\begin{tabular}{lrrr}
\hline atom & \multicolumn{1}{l}{$\mathrm{x}$} & $\mathrm{z}$ \\
\hline $\mathrm{C}$ & -3.00903 & -0.4456 & -0.51368 \\
$\mathrm{C}$ & -4.01181 & -1.33827 & -0.91997 \\
$\mathrm{C}$ & -1.8312 & -0.93076 & 0.268449 \\
$\mathrm{C}$ & -3.95601 & -2.6637 & -0.52902 \\
$\mathrm{C}$ & -1.92034 & -2.35104 & 0.713119 \\
$\mathrm{C}$ & -2.91331 & -3.17678 & 0.30074 \\
$\mathrm{C}$ & -1.75633 & 1.985803 & -0.22842 \\
$\mathrm{C}$ & -1.03423 & 1.420346 & 0.835451 \\
$\mathrm{C}$ & -1.44376 & 3.25398 & -0.73099 \\
$\mathrm{C}$ & 0.006299 & 2.167622 & 1.391649 \\
$\mathrm{C}$ & -0.39622 & 3.977852 & -0.16468 \\
$\mathrm{C}$ & 0.327083 & 3.433537 & 0.895929 \\
$\mathrm{C}$ & -1.39714 & 0.056393 & 1.399789 \\
$\mathrm{C}$ & 3.413832 & 0.154061 & -0.49596 \\
$\mathrm{~F}$ & 2.715408 & 1.206053 & -0.07044 \\
$\mathrm{~F}$ & 4.537774 & 0.0451 & 0.204684 \\
$\mathrm{~F}$ & 3.696164 & 0.294499 & -1.78852 \\
$\mathrm{H}$ & -4.84096 & -0.9851 & -1.52442 \\
$\mathrm{H}$ & -0.97714 & -0.92849 & -0.44637 \\
$\mathrm{H}$ & -4.7467 & -3.33422 & -0.85235 \\
$\mathrm{H}$ & -1.11207 & -2.71549 & 1.337234 \\
$\mathrm{H}$ & -2.93365 & -4.21898 & 0.598796 \\
$\mathrm{H}$ & -2.01359 & 3.670264 & -1.55625 \\
$\mathrm{H}$ & 0.57395 & 1.745728 & 2.212457 \\
$\mathrm{H}$ & -0.14943 & 4.959986 & -0.55468 \\
\hline & & &
\end{tabular}




\begin{tabular}{llll}
\hline $\mathrm{H}$ & 1.146525 & 3.989546 & 1.340226 \\
$\mathrm{H}$ & -2.22406 & 0.154672 & 2.113567 \\
$\mathrm{H}$ & -0.53309 & -0.84371 & 2.948432 \\
$\mathrm{H}$ & 1.234104 & -0.99191 & 1.516398 \\
$\mathrm{O}$ & -0.26812 & -0.48355 & 2.087199 \\
$\mathrm{O}$ & 3.253205 & -2.52467 & -0.58706 \\
$\mathrm{O}$ & 1.134982 & -1.17767 & -1.00188 \\
$\mathrm{O}$ & 2.139056 & -1.36851 & 1.292905 \\
$\mathrm{~S}$ & -3.17261 & 1.193745 & -0.96081 \\
$\mathrm{~S}$ & 2.390861 & -1.39616 & -0.28346 \\
\hline
\end{tabular}

Intermediate TS2

\begin{tabular}{|c|c|c|c|}
\hline atom & $\mathrm{X}$ & $\mathrm{y}$ & $\mathrm{z}$ \\
\hline $\mathrm{C}$ & -2.47955 & -0.80055 & -0.49302 \\
\hline $\mathrm{C}$ & -3.02001 & -1.92882 & -1.13132 \\
\hline $\mathrm{C}$ & -1.59304 & -0.96698 & 0.621048 \\
\hline $\mathrm{C}$ & -2.76635 & -3.20142 & -0.64021 \\
\hline $\mathrm{C}$ & -1.43106 & -2.29676 & 1.141478 \\
\hline $\mathrm{C}$ & -1.96807 & -3.39352 & 0.502269 \\
\hline $\mathrm{C}$ & -1.78299 & 1.86687 & -0.33847 \\
\hline $\mathrm{C}$ & -1.08911 & 1.545921 & 0.837713 \\
\hline $\mathrm{C}$ & -1.61872 & 3.116881 & -0.94902 \\
\hline $\mathrm{C}$ & -0.23188 & 2.508343 & 1.38745 \\
\hline $\mathrm{C}$ & -0.76657 & 4.061212 & -0.38337 \\
\hline $\mathrm{C}$ & -0.06932 & 3.75501 & 0.786419 \\
\hline $\mathrm{C}$ & -1.30176 & 0.222414 & 1.551991 \\
\hline $\mathrm{C}$ & 3.072604 & 0.259511 & -0.58762 \\
\hline $\mathrm{F}$ & 2.577674 & 1.291352 & 0.07902 \\
\hline $\mathrm{F}$ & 4.309942 & 0.000441 & -0.20153 \\
\hline $\mathrm{F}$ & 3.017513 & 0.469745 & -1.8914 \\
\hline $\mathrm{H}$ & -3.64759 & -1.7996 & -2.00736 \\
\hline $\mathrm{H}$ & -0.36598 & -1.02673 & -0.16057 \\
\hline $\mathrm{H}$ & -3.19454 & -4.05956 & -1.14893 \\
\hline $\mathrm{H}$ & -0.8182 & -2.43608 & 2.025361 \\
\hline $\mathrm{H}$ & -1.79045 & -4.39194 & 0.886063 \\
\hline $\mathrm{H}$ & -2.15276 & 3.343584 & -1.867 \\
\hline $\mathrm{H}$ & 0.309137 & 2.2711 & 2.295954 \\
\hline $\mathrm{H}$ & -0.64496 & 5.028694 & -0.86052 \\
\hline $\mathrm{H}$ & 0.599432 & 4.484166 & 1.232925 \\
\hline $\mathrm{H}$ & -2.13552 & 0.31724 & 2.258477 \\
\hline $\mathrm{H}$ & -0.3519 & -0.35547 & 3.237265 \\
\hline $\mathrm{H}$ & 1.097847 & -0.70087 & 1.755002 \\
\hline $\mathrm{O}$ & -0.11804 & -0.06285 & 2.340868 \\
\hline $\mathrm{O}$ & 2.667963 & -2.4506 & -0.67087 \\
\hline $\mathrm{O}$ & 0.661968 & -0.88501 & -0.79211 \\
\hline $\mathrm{O}$ & 1.902145 & -1.24706 & 1.36083 \\
\hline
\end{tabular}




\begin{tabular}{llll}
\hline $\mathrm{S}$ & -2.93362 & 0.762624 & -1.13304 \\
$\mathrm{~S}$ & 2.015625 & -1.25343 & -0.19592 \\
\hline
\end{tabular}

\begin{tabular}{lrrr} 
Product $\mathbf{F}$ & \multicolumn{3}{r}{} \\
\hline atom & $\mathrm{x}$ & $\mathrm{y}$ & \\
\hline $\mathrm{C}$ & 2.517829 & 1.822536 & 0.05082 \\
$\mathrm{C}$ & 2.125783 & 3.172313 & 0.037814 \\
$\mathrm{C}$ & 1.583359 & 0.808626 & 0.433823 \\
$\mathrm{C}$ & 0.831069 & 3.510483 & 0.395139 \\
$\mathrm{C}$ & 0.259046 & 1.200723 & 0.796444 \\
$\mathrm{C}$ & -0.10837 & 2.525003 & 0.775133 \\
$\mathrm{C}$ & 4.273417 & -0.26645 & -0.28503 \\
$\mathrm{C}$ & 3.177699 & -1.09033 & 0.126916 \\
$\mathrm{C}$ & 5.511826 & -0.84892 & -0.60674 \\
$\mathrm{C}$ & 3.378746 & -2.50181 & 0.199902 \\
$\mathrm{C}$ & 5.663364 & -2.22263 & -0.52139 \\
$\mathrm{C}$ & 4.595614 & -3.05631 & -0.11675 \\
$\mathrm{C}$ & 1.923364 & -0.5536 & 0.458468 \\
$\mathrm{C}$ & -3.47078 & 0.611248 & -0.38282 \\
$\mathrm{~F}$ & -3.14495 & 0.997162 & 0.853509 \\
$\mathrm{~F}$ & -2.36558 & 0.448043 & -1.10482 \\
$\mathrm{~F}$ & -4.25842 & 1.514305 & -0.94778 \\
$\mathrm{H}$ & 2.832888 & 3.94323 & -0.25117 \\
$\mathrm{H}$ & -5.56319 & -0.80601 & 1.477949 \\
$\mathrm{H}$ & 0.535466 & 4.55493 & 0.381104 \\
$\mathrm{H}$ & -0.43456 & 0.417598 & 1.086583 \\
$\mathrm{H}$ & -1.11738 & 2.817176 & 1.044614 \\
$\mathrm{H}$ & 6.342593 & -0.22469 & -0.91986 \\
$\mathrm{H}$ & 2.544169 & -3.12168 & 0.512086 \\
$\mathrm{H}$ & 6.622647 & -2.665 & -0.77113 \\
$\mathrm{H}$ & 4.74182 & -4.12945 & -0.05871 \\
$\mathrm{H}$ & 1.135756 & -1.24411 & 0.761759 \\
$\mathrm{H}$ & -0.90354 & -2.00162 & 2.42603 \\
$\mathrm{H}$ & -1.59599 & -2.05925 & 1.061133 \\
O & -0.71572 & -2.02194 & 1.474981 \\
O & -4.76411 & -1.38685 & -1.6378 \\
O & -5.67913 & -0.61344 & 0.52408 \\
O & -3.53356 & -1.9215 & 0.532531 \\
$\mathrm{~S}$ & 4.150777 & 1.460346 & -0.4123 \\
$\mathrm{~S}$ & -4.35402 & -1.03736 & -0.29266 \\
\hline & & & \\
& & &
\end{tabular}




\section{References}

(1) (a) Arai, T.; Yamamoto, Y. Org. Lett. 2014, 16, 1700-1703. (b) Arai, T.; Miyazaki, T.; Ogawa, H.; Masu, H. 2016, 18, 5824-5827. (c) Soeta, T.; Shitaya, S.; Okuno, T.; Fujinami, S.; Ukaji, Y. Tetrahedron 2016, 72, 7901-7905. (d) Simlandy, A. K.; Mukherjee, S. J. Org. Chem. 2017, 82, 4851-4858.

(2) Gaussian 16, Revision A.03, Frisch, M. J.; Trucks, G. W.; Schlegel, H. B.; Scuseria, G. E.; Robb, M. A.; Cheeseman, J. R.; Scalmani, G.; Barone, V.; Petersson, G. A.; Nakatsuji, H.; Li, X.; Caricato, M.; Marenich, A. V.; Bloino, J.; Janesko, B. G.; Gomperts, R.; Mennucci, B.; Hratchian, H. P.; Ortiz, J. V.; Izmaylov, A. F.; Sonnenberg, J. L.; Williams-Young, D.; Ding, F.; Lipparini, F.; Egidi, F.; Goings, J.; Peng, B.; Petrone, A.; Henderson, T.; Ranasinghe, D.; Zakrzewski, V. G.; Gao, J.; Rega, N.; Zheng, G.; Liang, W.; Hada, M.; Ehara, M.; Toyota, K.; Fukuda, R.; Hasegawa, J.; Ishida, M.; Nakajima, T.; Honda, Y.; Kitao, O.; Nakai, H.; Vreven, T.; Throssell, K.; Montgomery, Jr., J. A.; Peralta, J. E.; Ogliaro, F.; Bearpark, M. J.; Heyd, J. J.; Brothers, E. N.; Kudin, K. N.; Staroverov, V. N.; Keith, T. A.; Kobayashi, R.; Normand, J.; Raghavachari, K.; Rendell, A. P.; Burant, J. C.; Iyengar, S. S.; Tomasi, J.; Cossi, M.; Millam, J. M.; Klene, M.; Adamo, C.; Cammi, R.; Ochterski, J. W.; Martin, R. L.; Morokuma, K.; Farkas, O.; Foresman, J. B.; Fox, D. J. Gaussian, Inc., Wallingford CT, 2016.

(3) Becke, A. D. Density-functional thermochemistry. III. The role of exact exchange. J. Chem. Phys. 1993, 98, 5648-5652.

(4) Yu, H. S.; He, X.; Li, S. L.; Truhlar, D. G. MN15: A Kohn-Sham Global-Hybrid Exchange-Correlation Density Functional with Broad Accuracy for Multi-Reference and Single-Reference Systems and Noncovalent Interactions. Chem. Sci. 2016, 7, 5032-5051.

(5) Peng C.; Ayala,P. Y.; Schlegel, H. B.; Frisch, M. J. Using redundant internal coordinates to optimize equilibrium geometries and transition states. J. Comp. Chem. 1996, 17, 49-56.

(6) Gonzalez, C.; Schlegel, H. B. An improved algorithm for reaction path following. J. Chem. Phys. 1989, 90, 2154-2161.

(7) Tomasi, J.; Mennucci, B.; Cammi, R. Quantum mechanical continuum solvation models. Chem. Rev. 2005, 105, 2999-3093.

(8) Simon, S.; Duran, M.; Dannenberg, J. J. How does basis set superposition error change the potential surfaces for hydrogen-bonded dimers? J. Chem. Phys. 1996, 105, 11024-11031. 
${ }^{1} \mathrm{H}$ and ${ }^{13} \mathrm{C}$ NMR Spectra of All New Compounds 
${ }^{1} \mathrm{H}$ NMR (400 MHz, $\mathrm{CDCl}_{3}, 298 \mathrm{~K}$ )
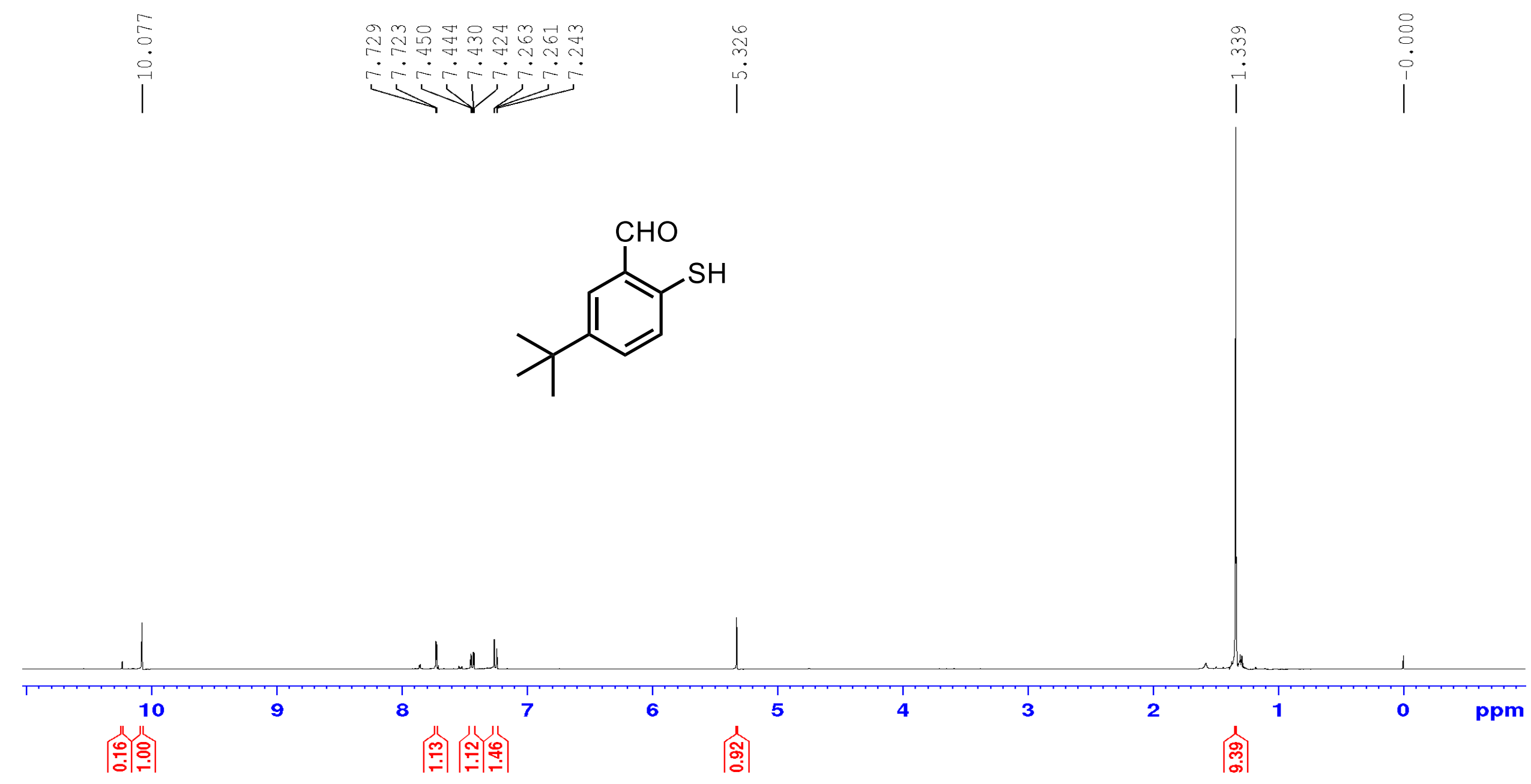
${ }^{13} \mathrm{C}\left\{{ }^{1} \mathrm{H}\right\}$ NMR (101 MHz, $\mathrm{CDCl}$, $\left.298 \mathrm{~K}\right)$
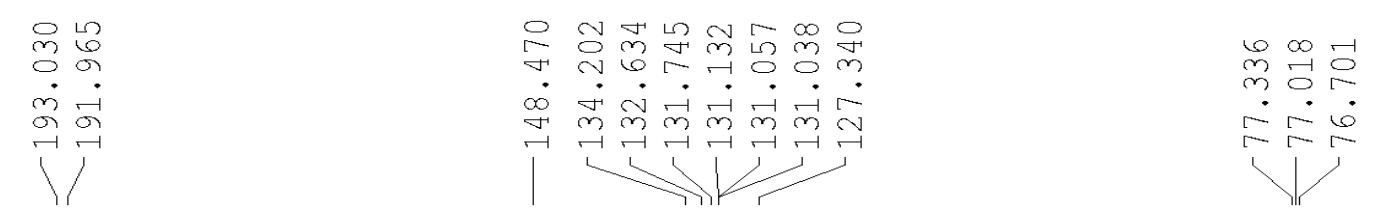

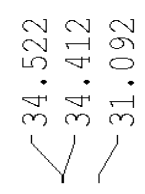<smiles>CC(C)(C)c1ccc(S)c(C=O)c1</smiles>

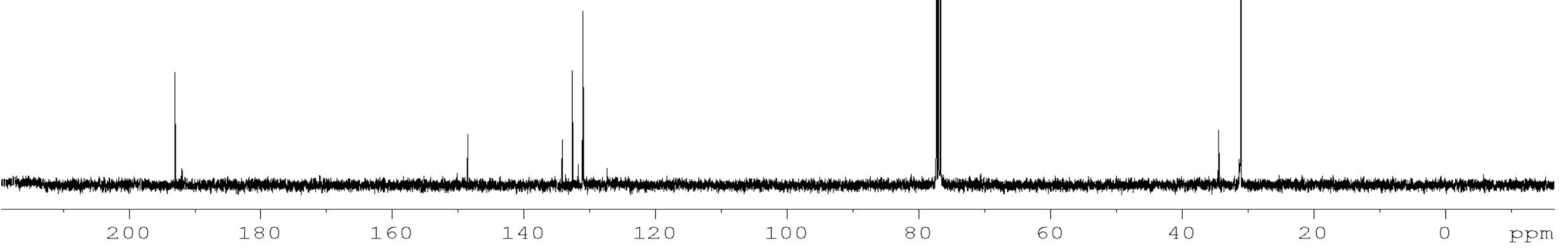


${ }^{1} \mathrm{H}$ NMR (400 MHz, $\mathrm{CDCl}_{3}, 298 \mathrm{~K}$ )

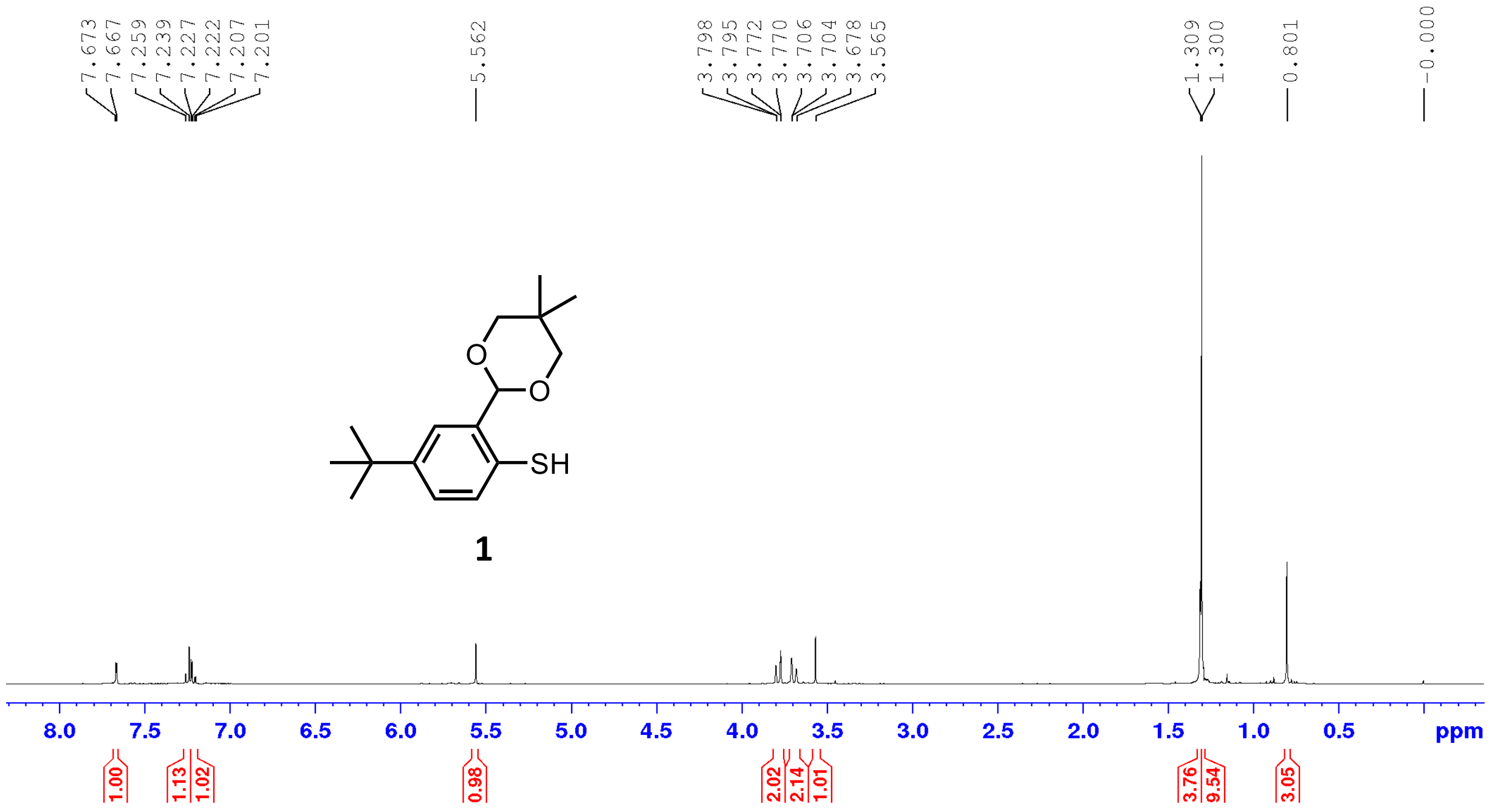


${ }^{13} \mathrm{C}\left\{{ }^{1} \mathrm{H}\right\} \mathrm{NMR}\left(101 \mathrm{MHz}, \mathrm{CDCl}_{3}, 298 \mathrm{~K}\right)$

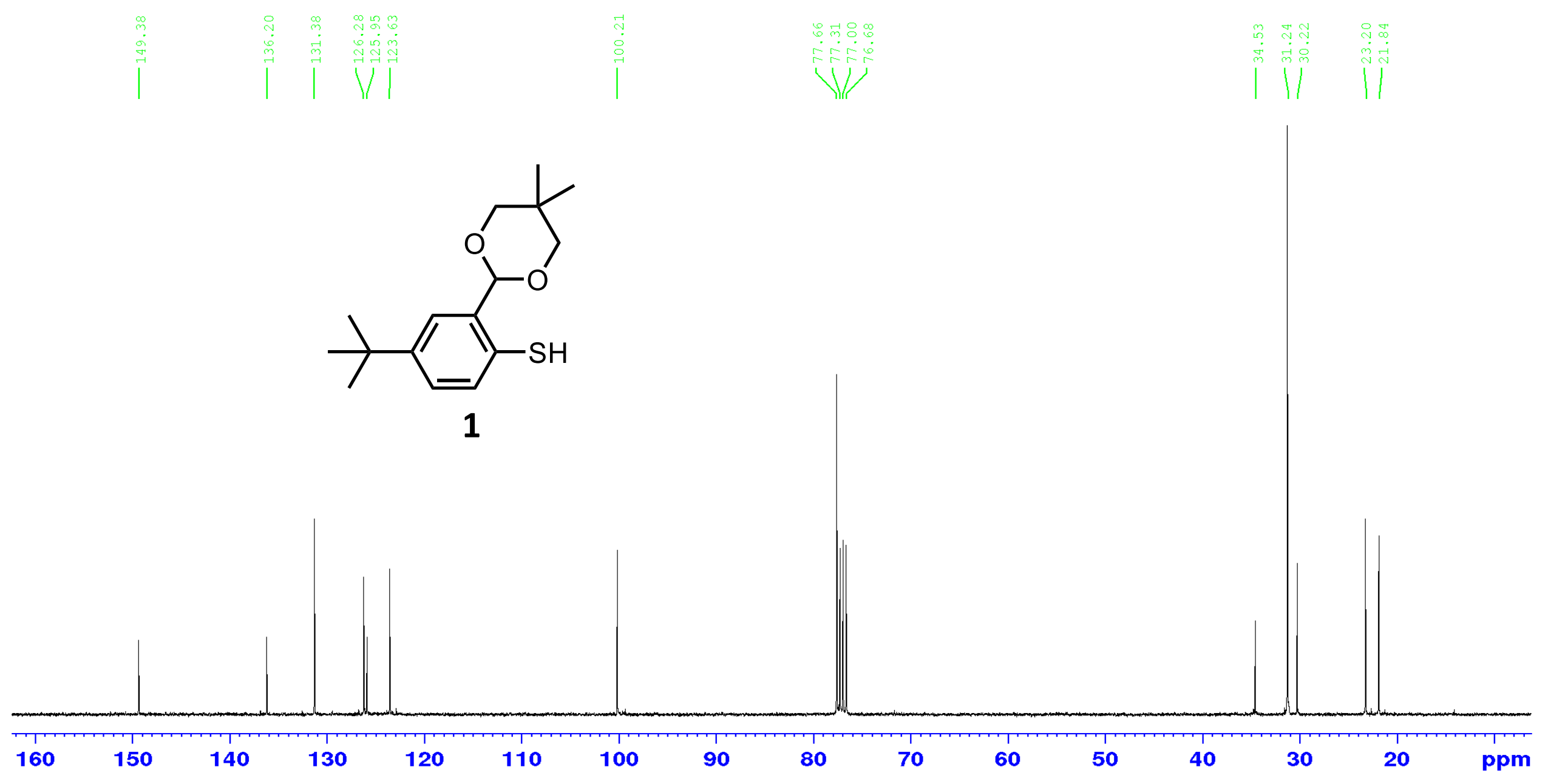


${ }^{1} \mathrm{H}$ NMR (400 MHz, $\mathrm{CDCl}_{3}, 298 \mathrm{~K}$ )

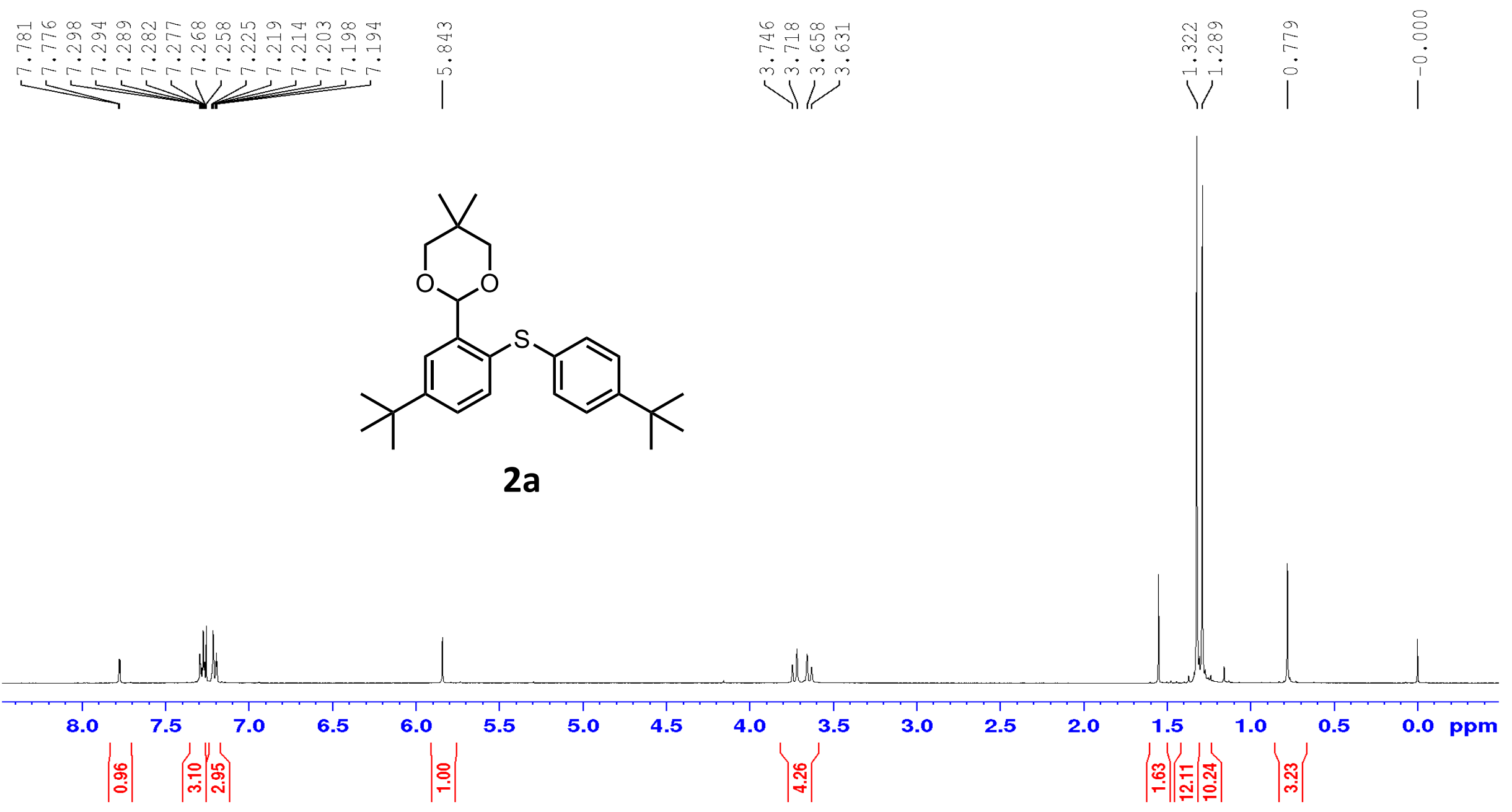


${ }^{13} \mathrm{C}\left\{{ }^{1} \mathrm{H}\right\} \mathrm{NMR}\left(101 \mathrm{MHz}, \mathrm{CDCl}_{3}, 298 \mathrm{~K}\right)$
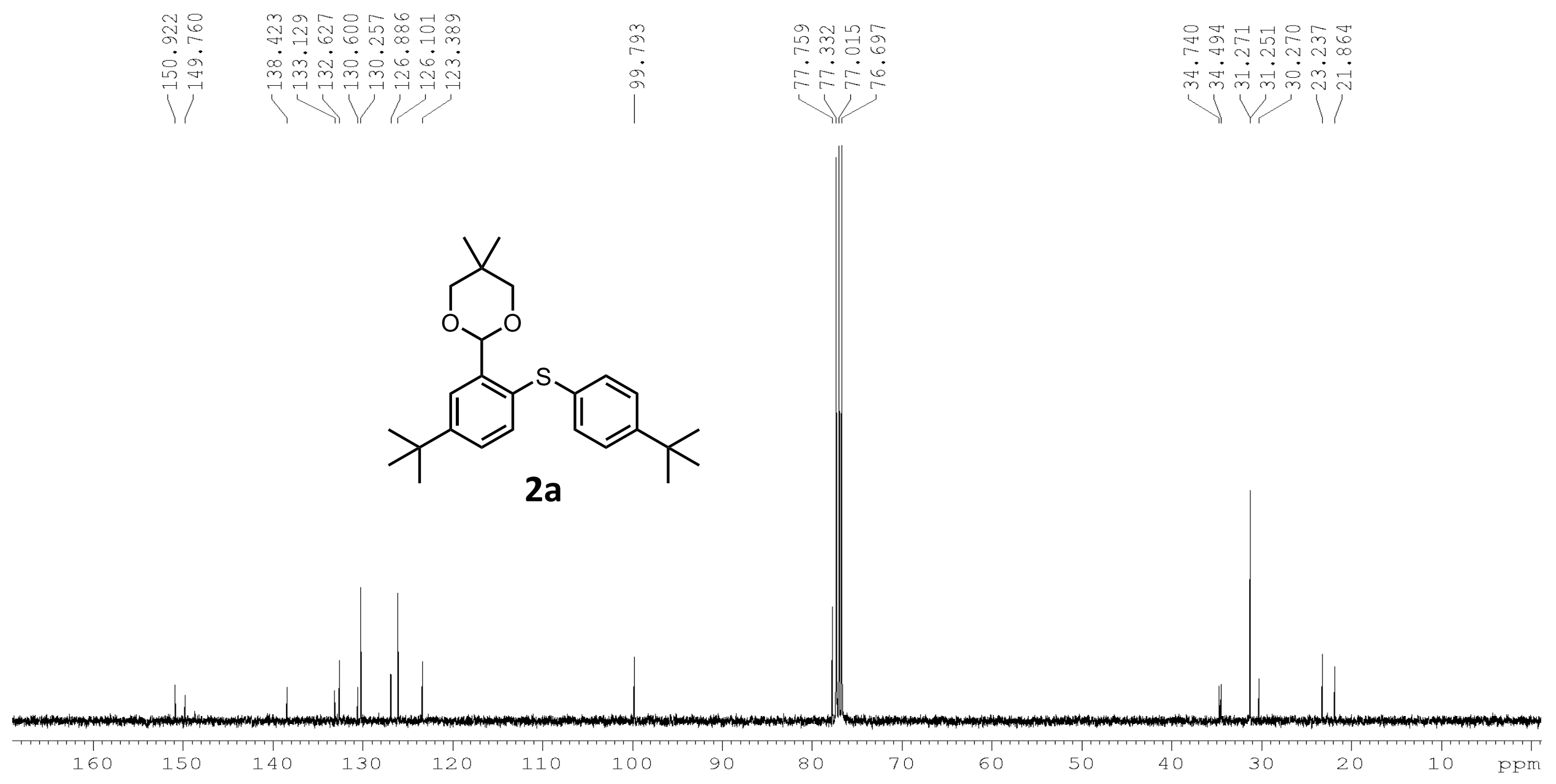
${ }^{1} \mathrm{H}$ NMR (400 MHz, $\mathrm{CDCl}_{3}, 298 \mathrm{~K}$ )
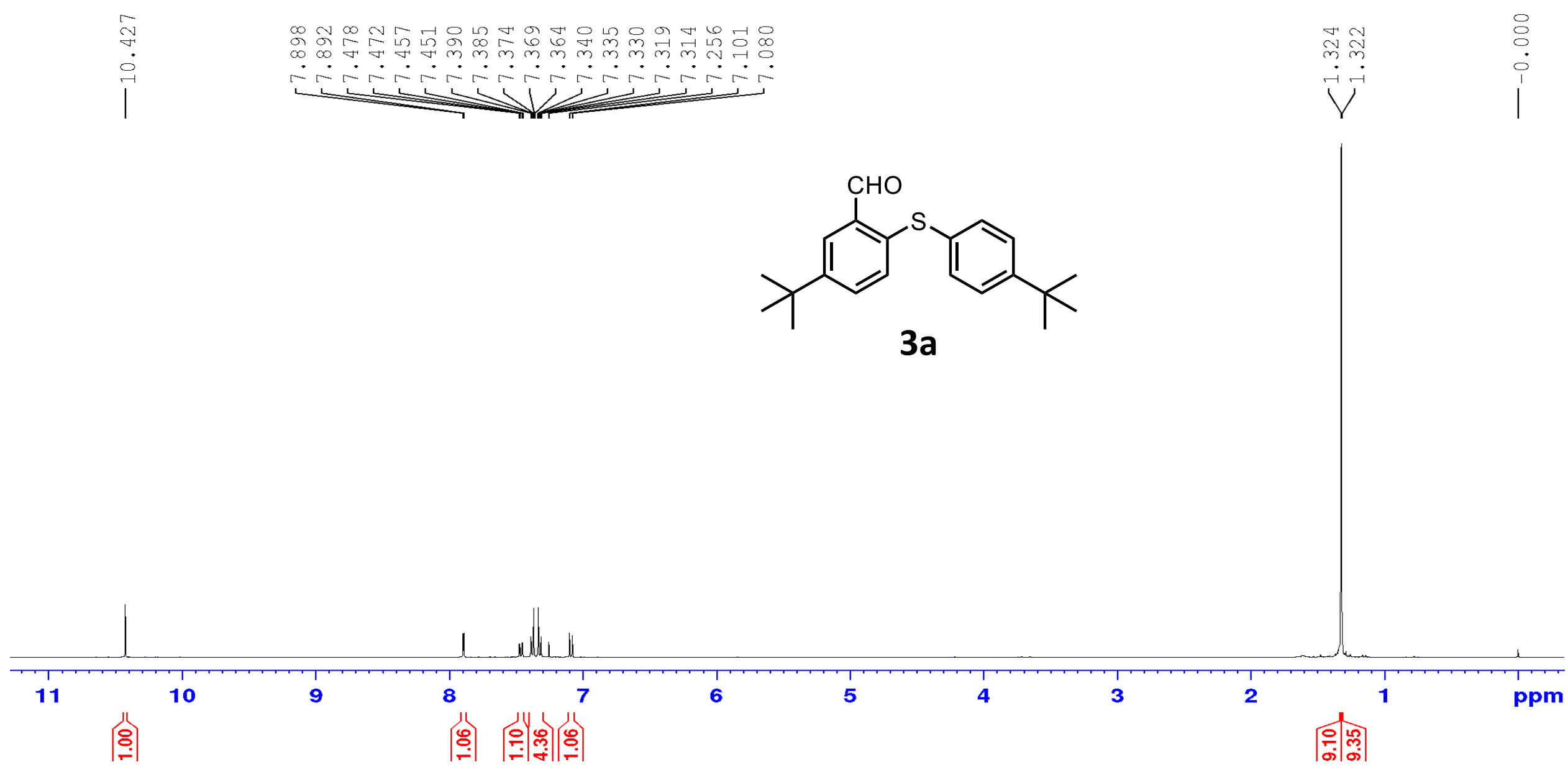
${ }^{13} \mathrm{C}\left\{{ }^{1} \mathrm{H}\right\}$ NMR (101 MHz, $\mathrm{CDCl}$, $\left.298 \mathrm{~K}\right)$
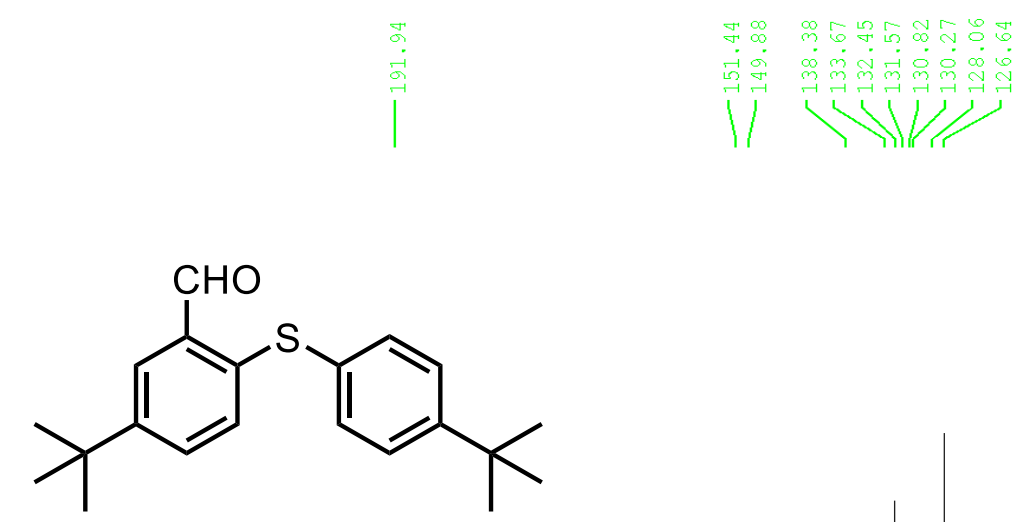

$3 a$

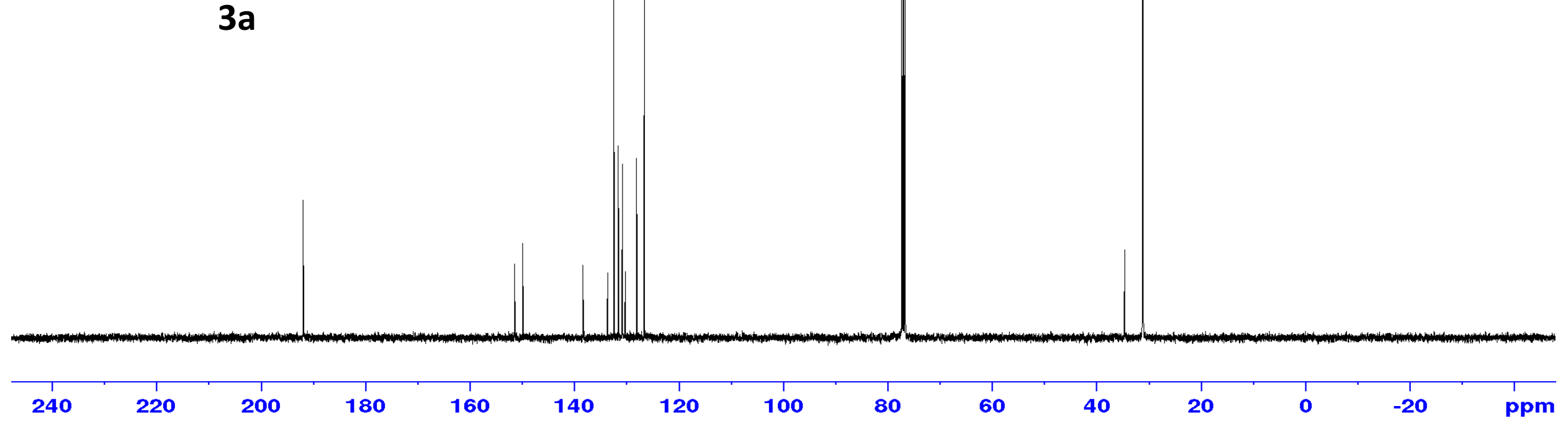


${ }^{1} \mathrm{H}$ NMR (400 MHz, CD ${ }_{3} \mathrm{CN}, 298 \mathrm{~K}$ )

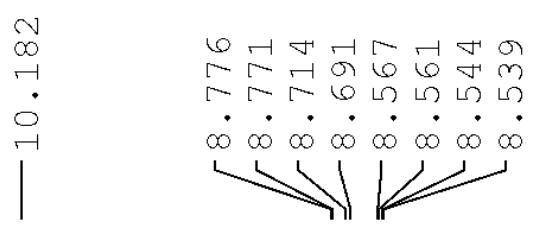

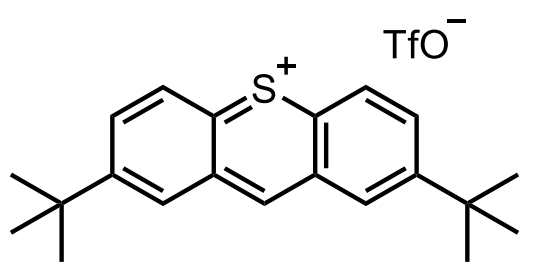

$4 a$

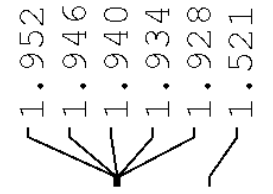

เn t

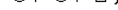

w
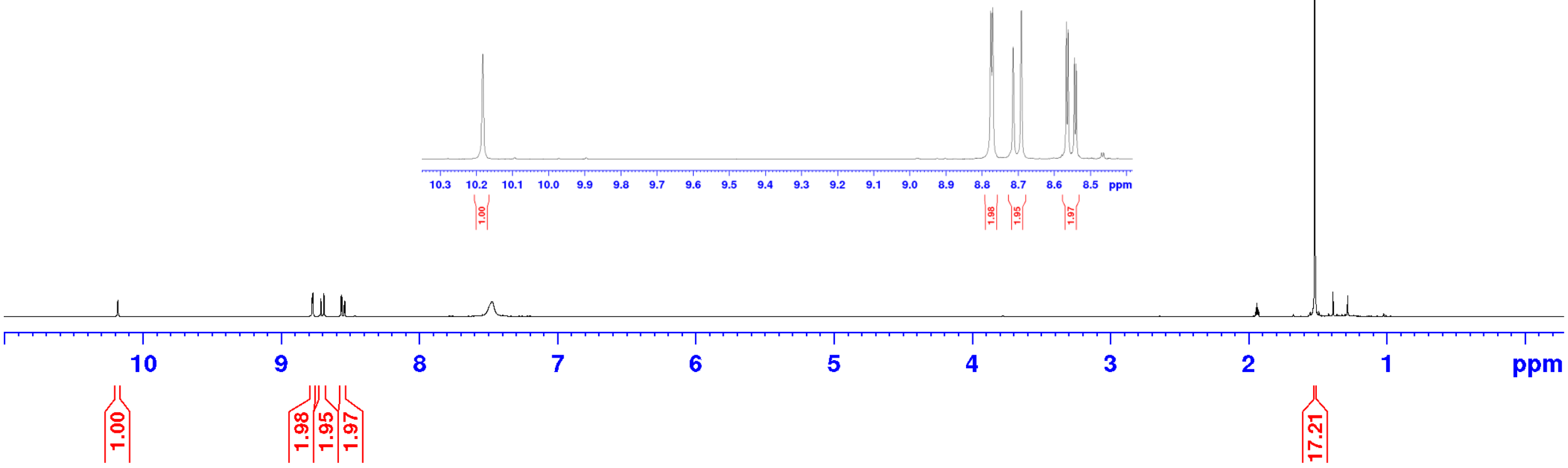
${ }^{1} \mathrm{H} \mathrm{NMR}\left(400 \mathrm{MHz}, \mathrm{CDCl}_{3}, 298 \mathrm{~K}\right)$

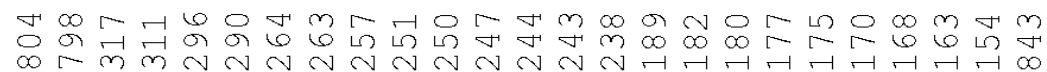

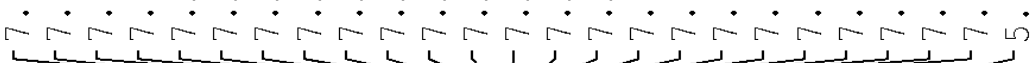

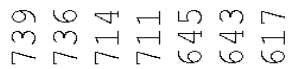

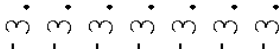

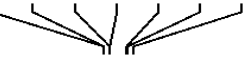<smiles>CC1(C)COC(c2cc(C(C)(C)C)ccc2Sc2ccccc2)OC1</smiles>

$2 b$

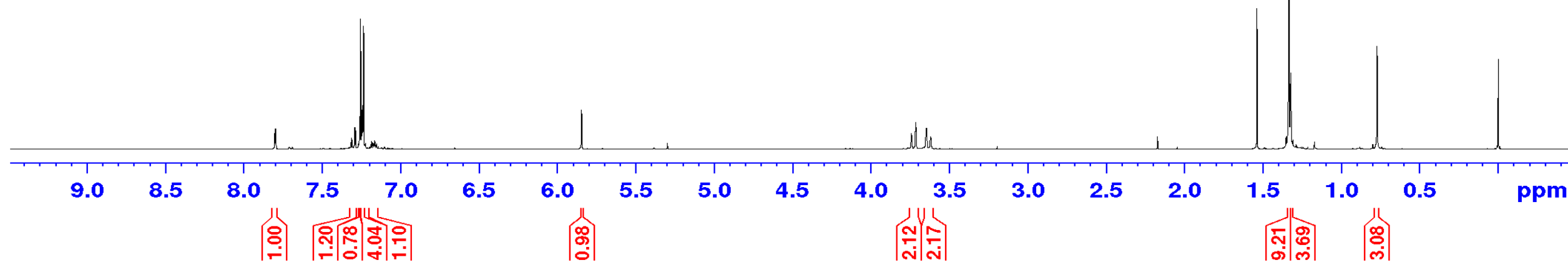


${ }^{13} \mathrm{C}\left\{{ }^{1} \mathrm{H}\right\} \mathrm{NMR}\left(101 \mathrm{MHz}, \mathrm{CDCl}_{3}, 298 \mathrm{~K}\right)$

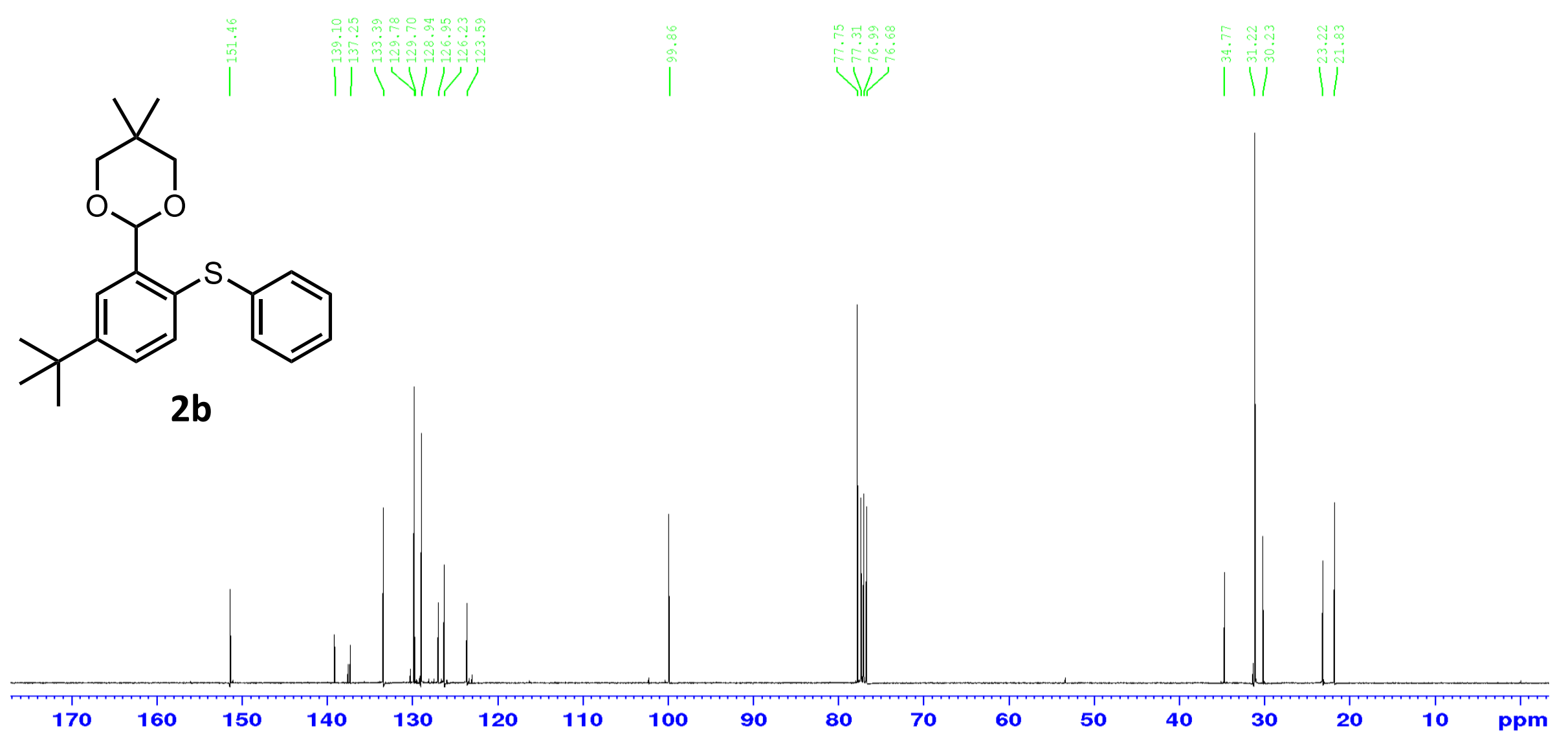


${ }^{1} \mathrm{H}$ NMR (400 MHz, $\mathrm{CDCl}_{3}, 298 \mathrm{~K}$ )
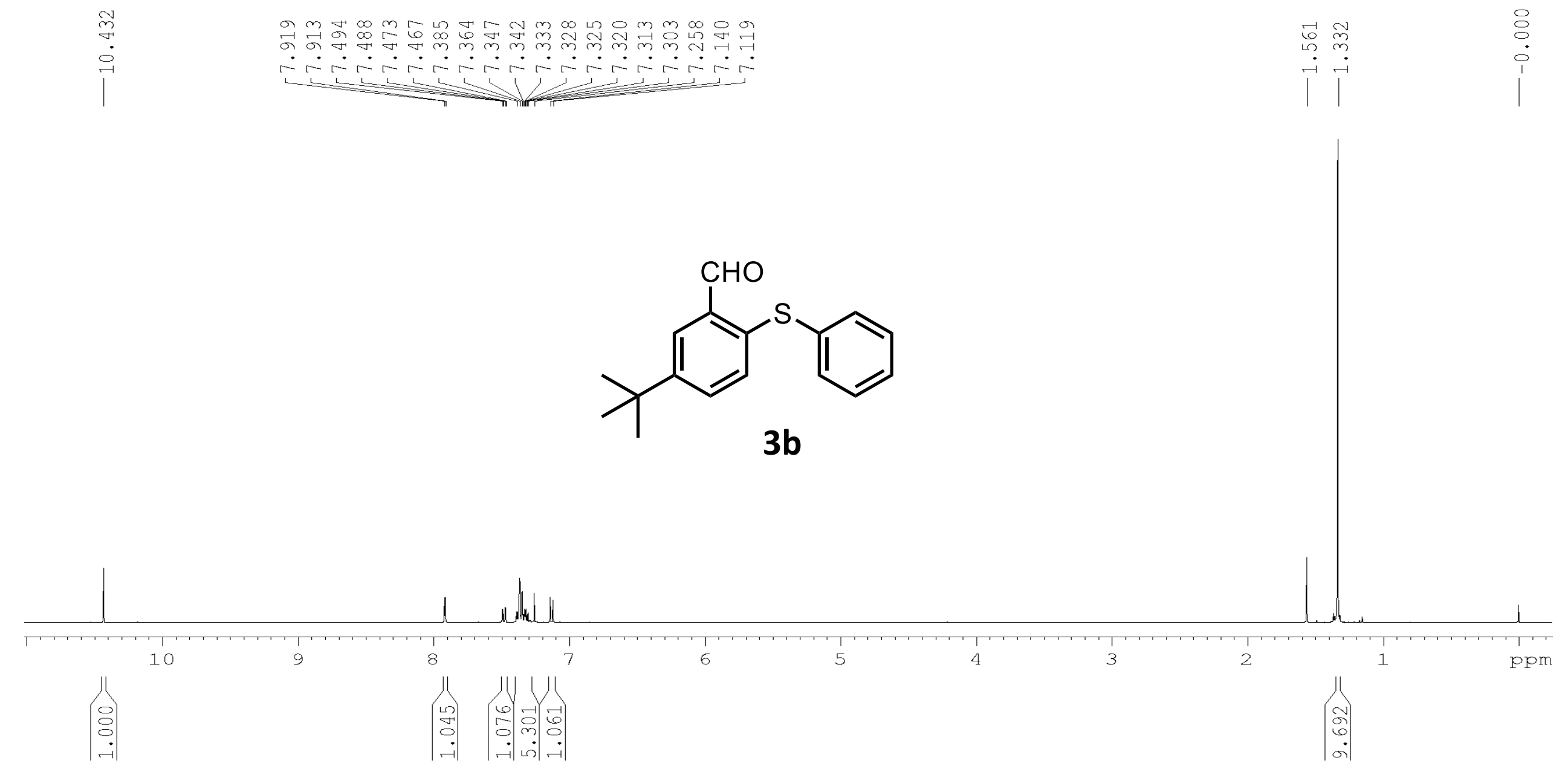
${ }^{13} \mathrm{C}\left\{{ }^{1} \mathrm{H}\right\} \mathrm{NMR}\left(101 \mathrm{MHz}, \mathrm{CDCl}_{3}, 298 \mathrm{~K}\right)$
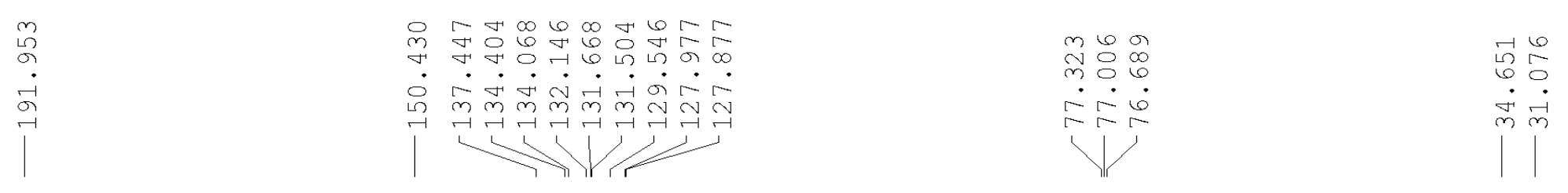<smiles>CC(C)(C)c1ccc(Sc2ccccc2)c(C=O)c1</smiles>

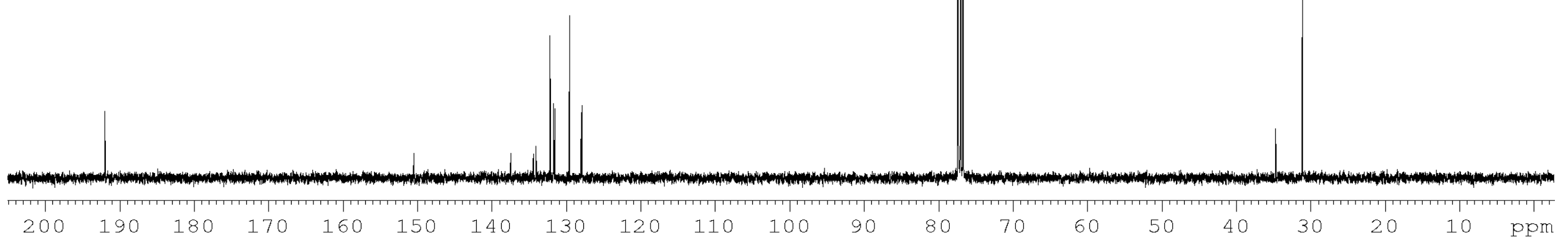


${ }^{1} \mathrm{H}$ NMR (400 MHz, $\mathrm{CD}_{3} \mathrm{CN}, 298 \mathrm{~K}$ )

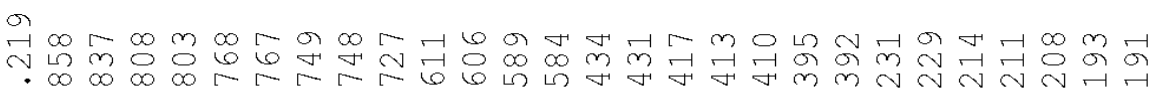

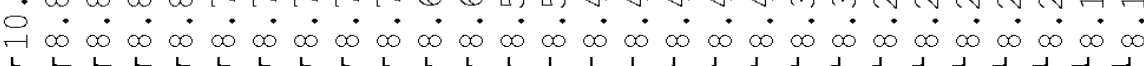

-

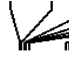

4b
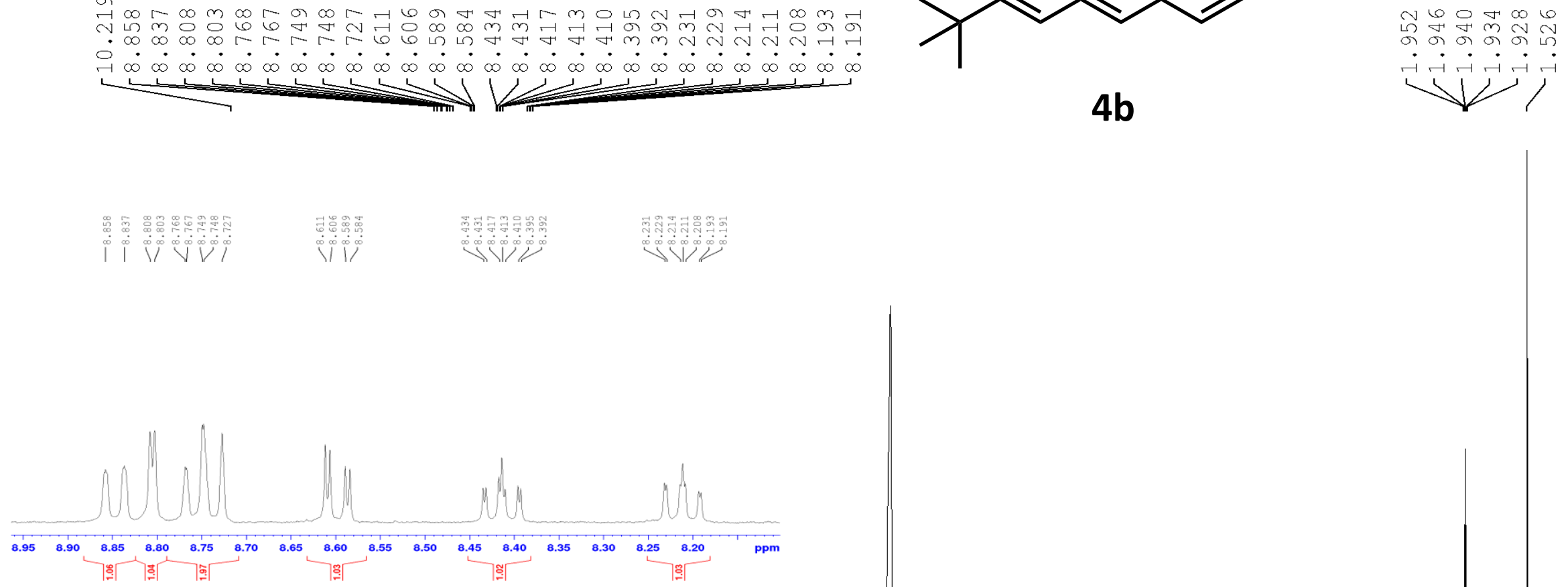

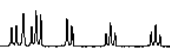
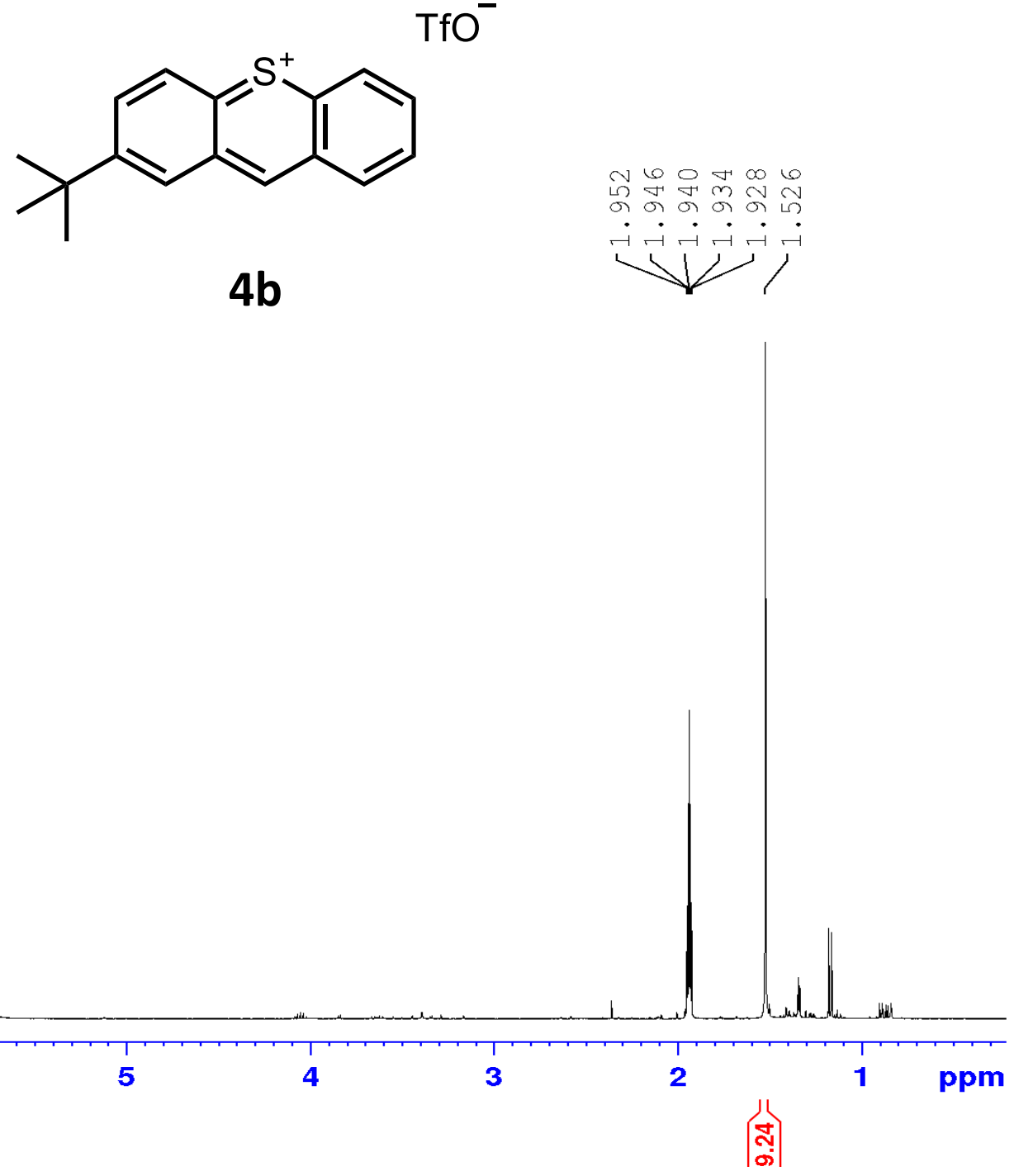


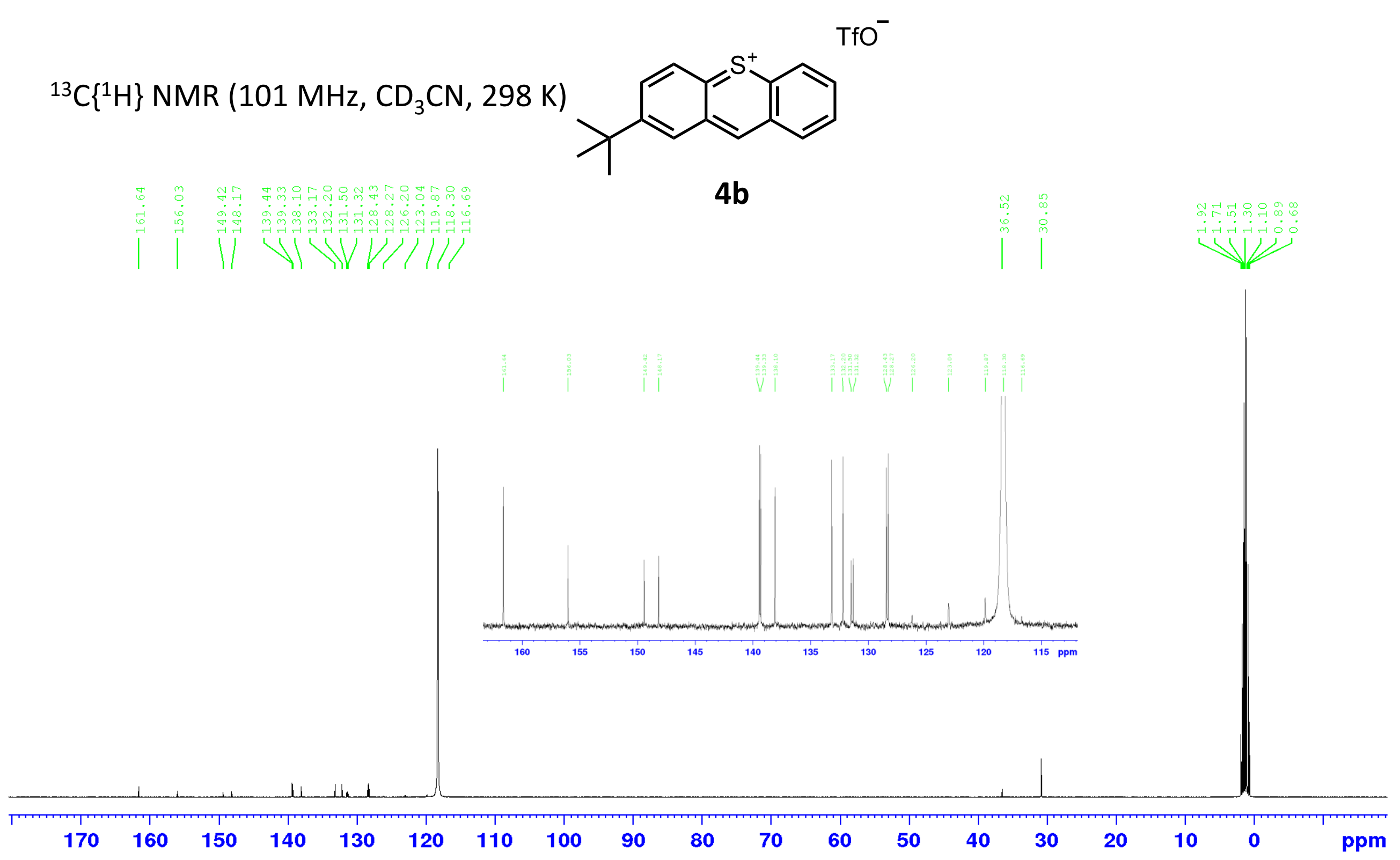


${ }^{1} \mathrm{H}$ NMR (400 MHz, $\left.\mathrm{CDCl}_{3}, 298 \mathrm{~K}\right)$
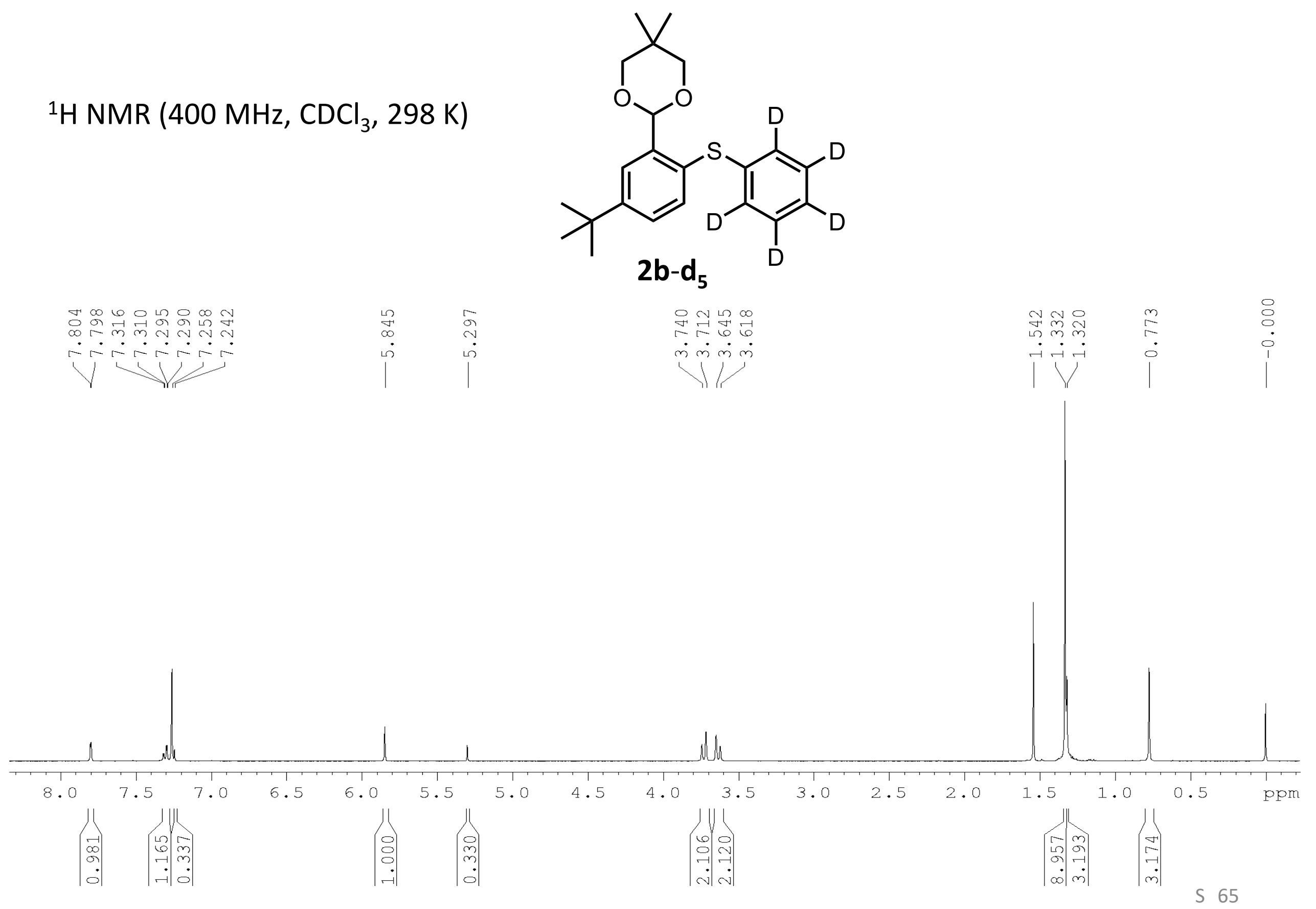
${ }^{13} \mathrm{C}\left\{{ }^{1} \mathrm{H}\right\}$ NMR (101 MHz, $\left.\mathrm{CDCl}, 298 \mathrm{~K}\right)$

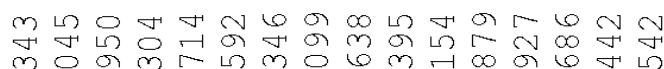

- 00 .

क्या-

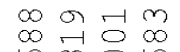

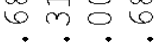

FER<smiles>[2H]c1c([2H])c([2H])c(Sc2ccc(C(C)(C)C)cc2C2OCC(C)(C)CO2)c([2H])c1[2H]</smiles>

(1) Anf

$2 b-d_{5}$

गें

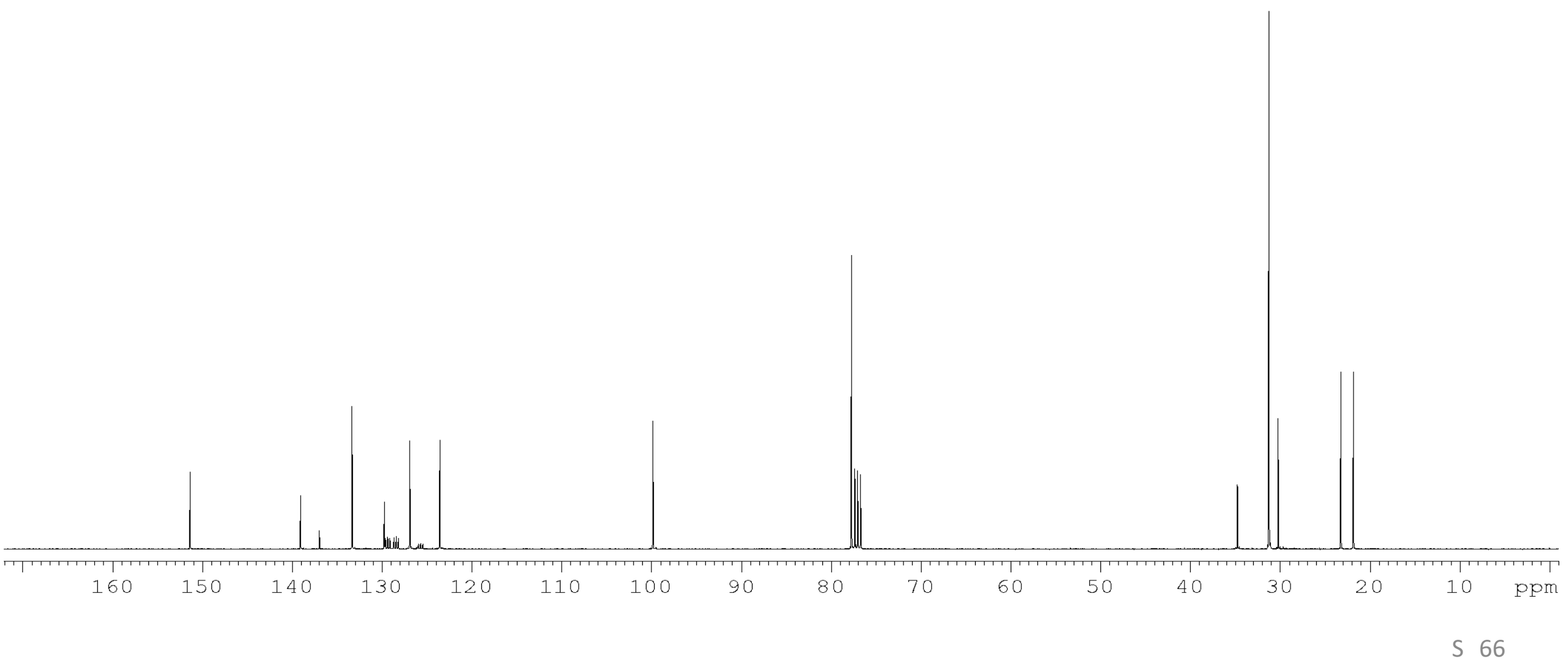


${ }^{1} \mathrm{H}$ NMR (400 MHz, $\mathrm{CDCl}_{3}, 298 \mathrm{~K}$ )
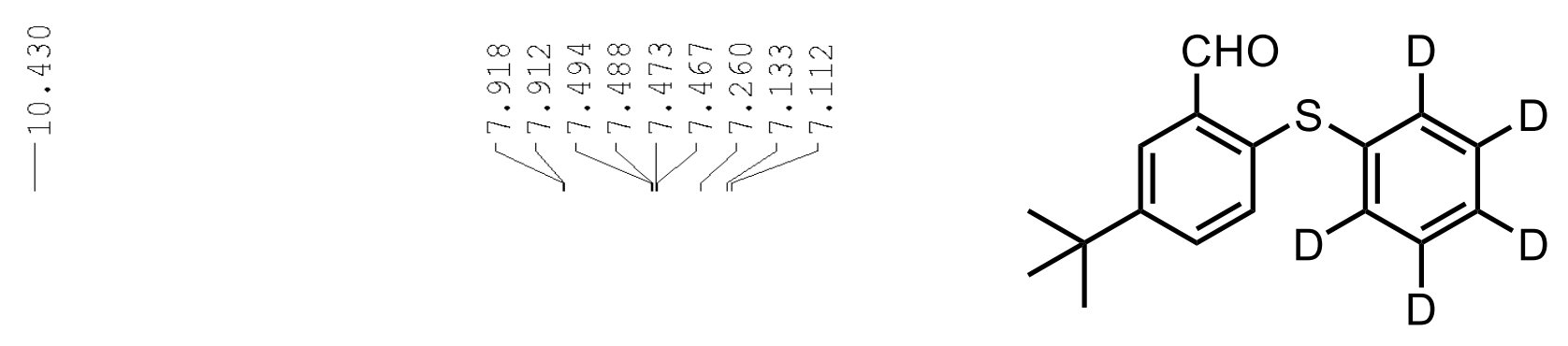

$3 b-d_{5}$

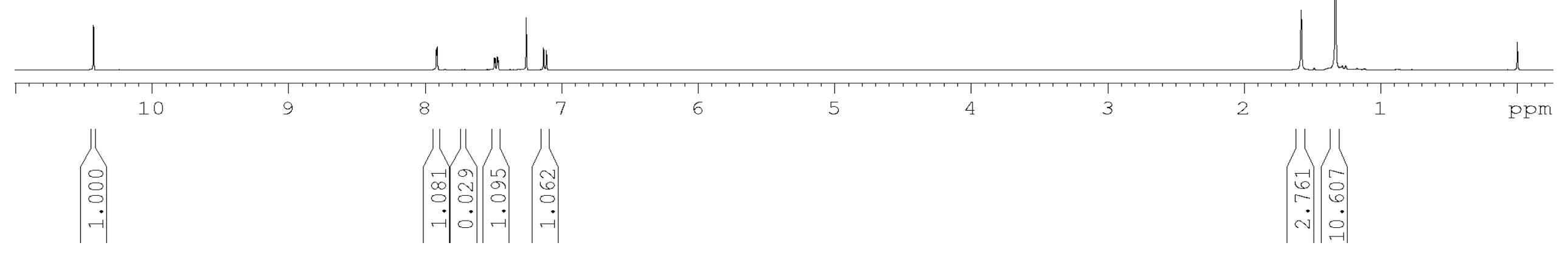


${ }^{13} \mathrm{C}\left\{{ }^{1} \mathrm{H}\right\} \mathrm{NMR}\left(101 \mathrm{MHz}, \mathrm{CDCl}_{3}, 298 \mathrm{~K}\right)$

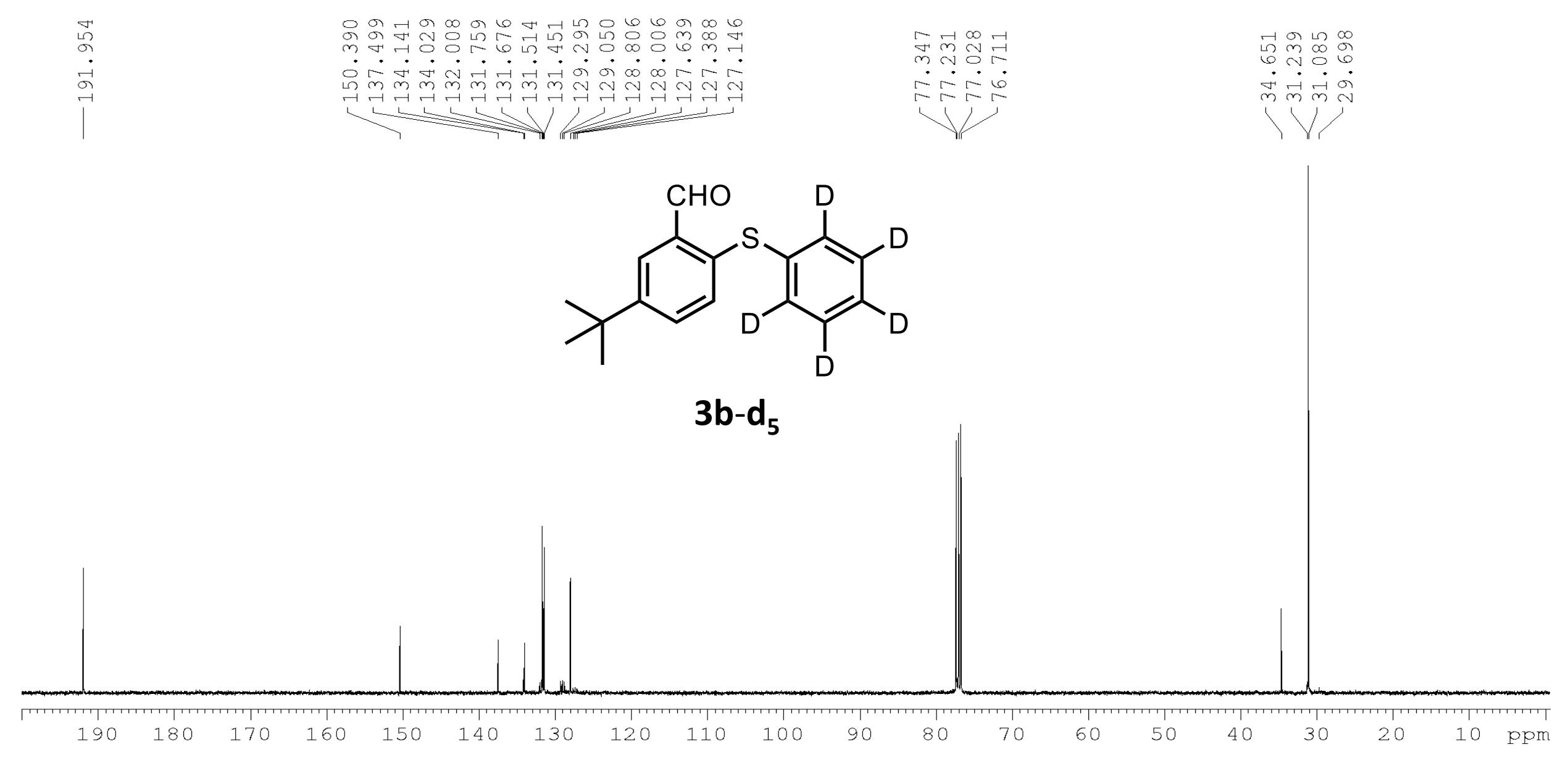


${ }^{1} \mathrm{H}$ NMR (400 MHz, CD 3 CN, $298 \mathrm{~K}$ )

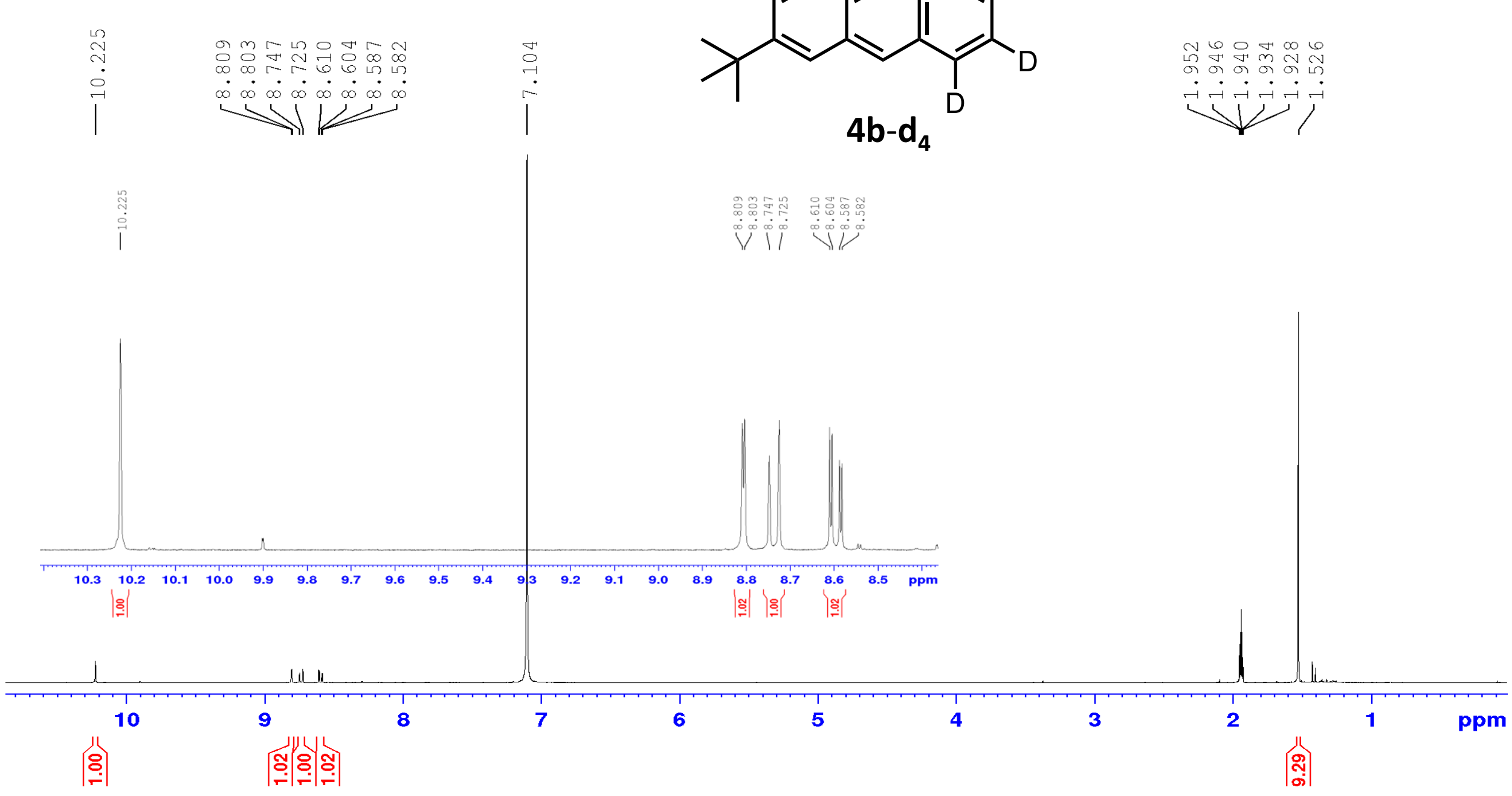


${ }^{13} \mathrm{C}\left\{{ }^{1} \mathrm{H}\right\}$ NMR (101 MHz, CD 3 CN, $\left.298 \mathrm{~K}\right)$
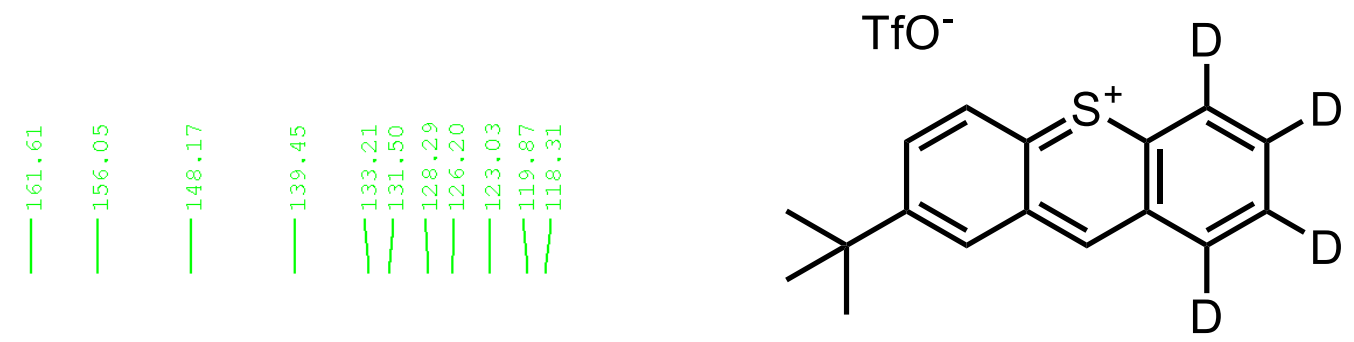

$4 b-d_{4}$

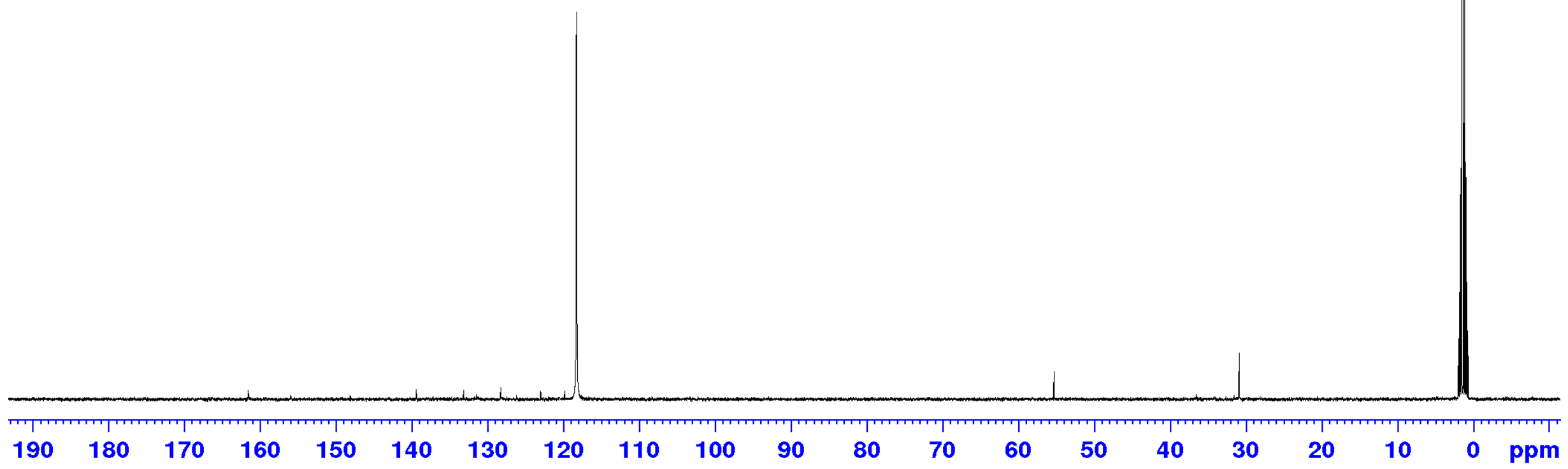


${ }^{1} \mathrm{H}$ NMR (400 MHz, $\mathrm{CDCl}_{3}, 298 \mathrm{~K}$ )
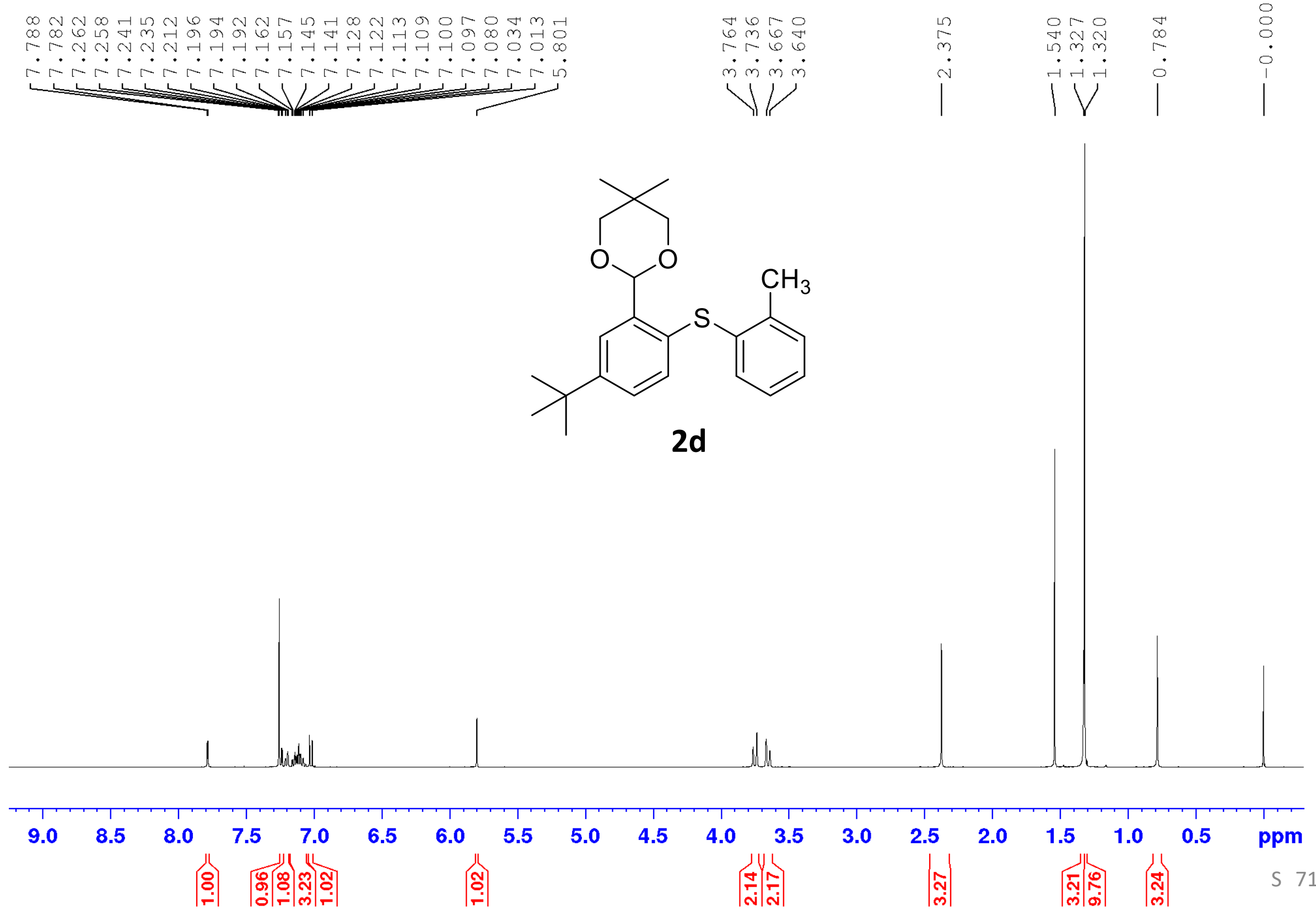
${ }^{13} \mathrm{C}\left\{{ }^{1} \mathrm{H}\right\}$ NMR (101 MHz, $\left.\mathrm{CDCl}_{3}, 298 \mathrm{~K}\right)$

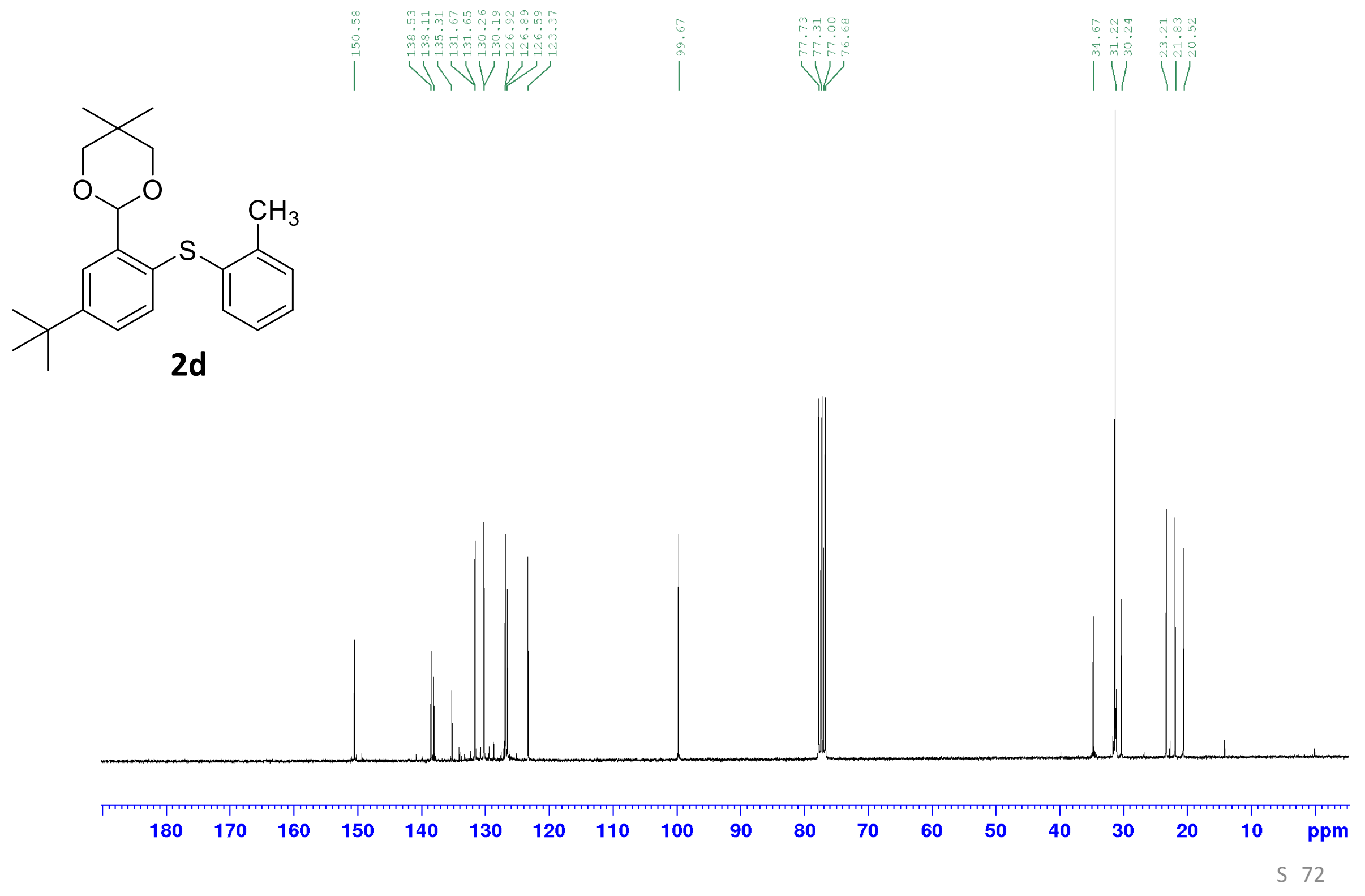


${ }^{1} \mathrm{H}$ NMR (400 MHz, $\mathrm{CDCl}_{3}, 298 \mathrm{~K}$ )
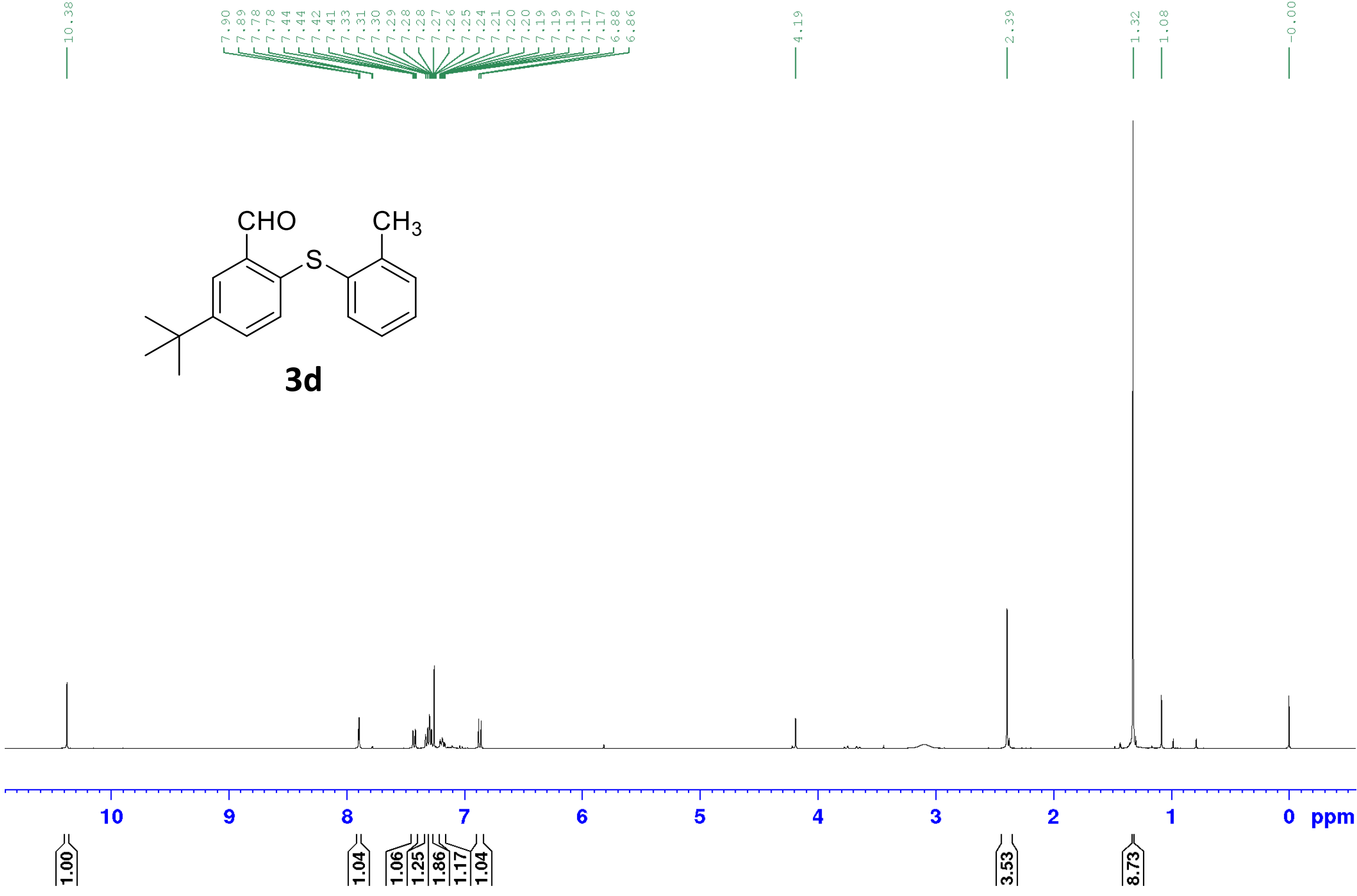
${ }^{13} \mathrm{C}\left\{{ }^{1} \mathrm{H}\right\}$ NMR (101 MHz, CDCl, $\left.298 \mathrm{~K}\right)$
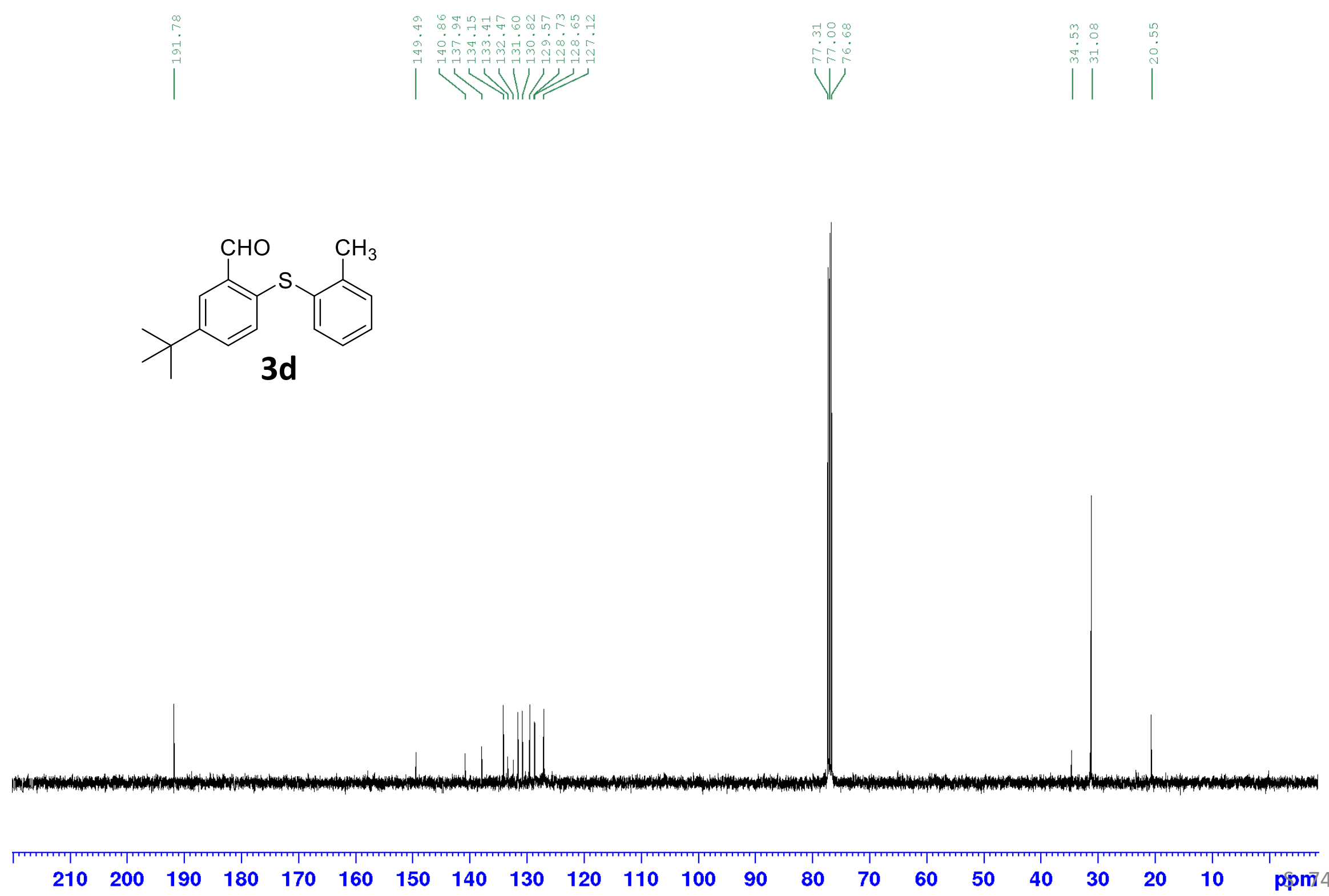
${ }^{1} \mathrm{H}$ NMR (400 MHz, CD 3 CN, $298 \mathrm{~K}$ )
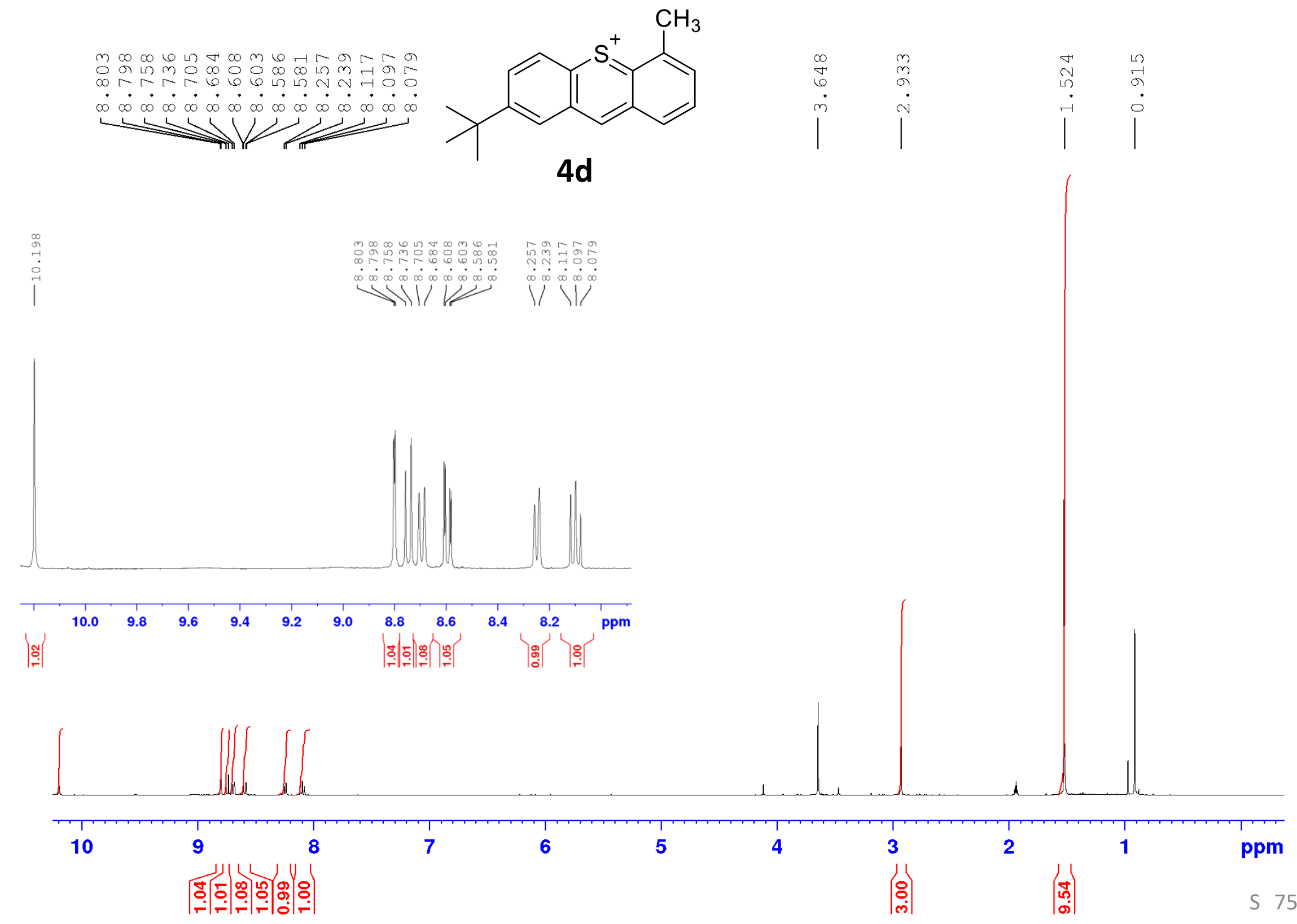

$\mathrm{TfO}^{-}$ 
${ }^{13} \mathrm{C}\left\{{ }^{1} \mathrm{H}\right\}$ NMR $\left(101 \mathrm{MHz}, \mathrm{CD}_{3} \mathrm{CN}, 298 \mathrm{~K}\right)$
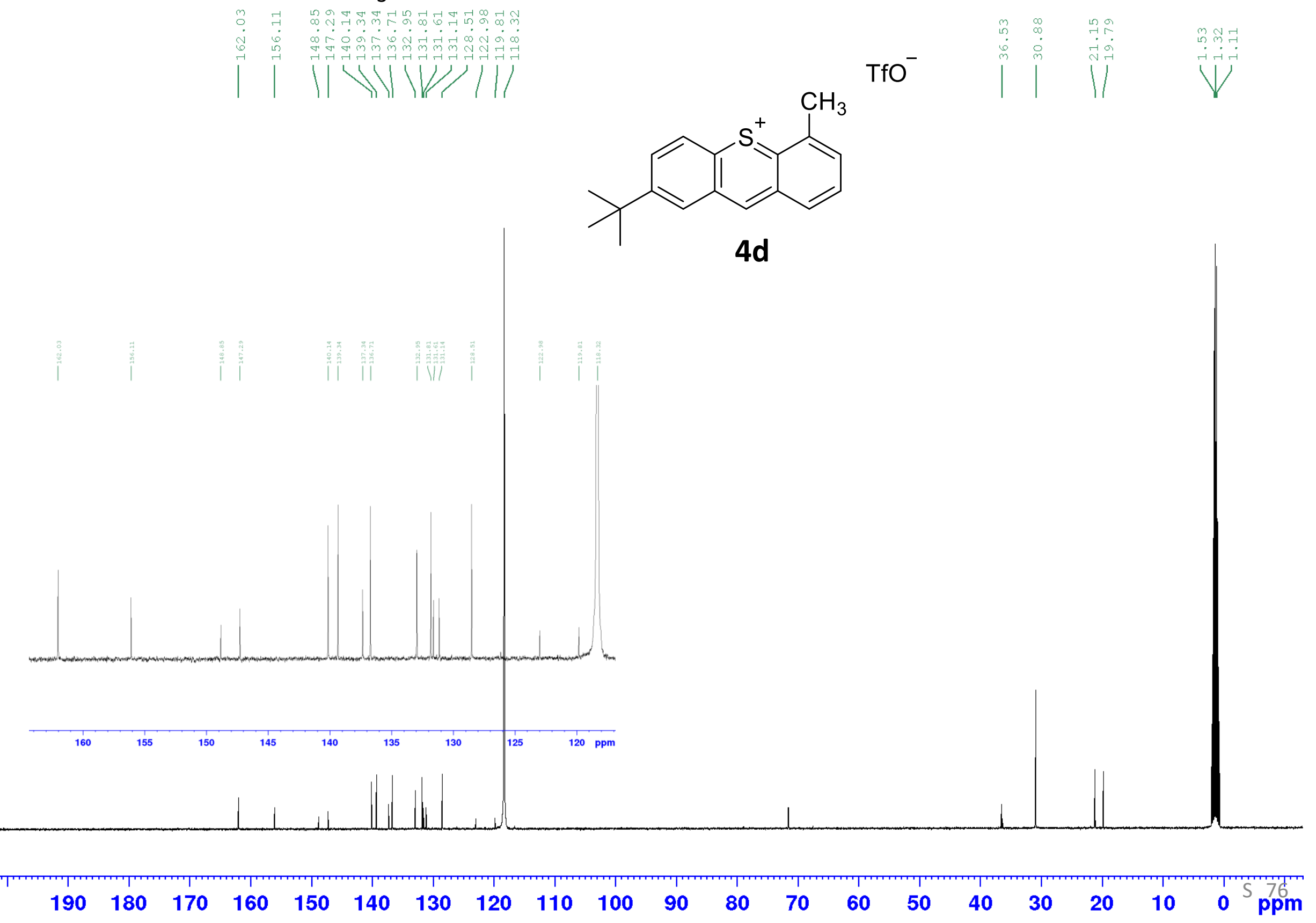
${ }^{1} \mathrm{H}$ NMR (400 MHz, $\left.\mathrm{CDCl}_{3}, 298 \mathrm{~K}\right)$
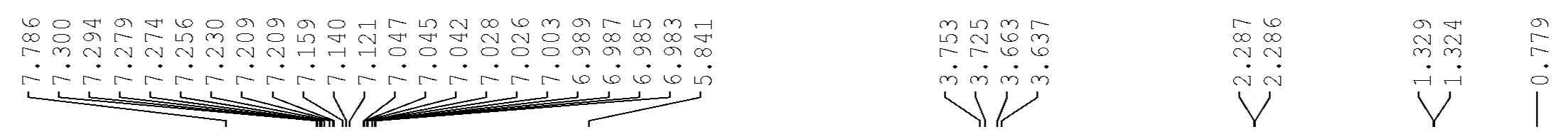

8
8
0
1
1<smiles>Cc1cccc(Sc2ccc(C(C)(C)C)cc2C2OCC(C)(C)CO2)c1</smiles>

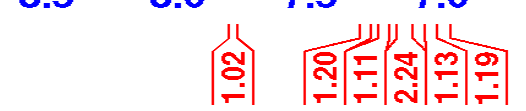

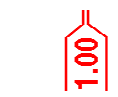

會

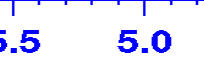

$4.5 \quad 4.0$
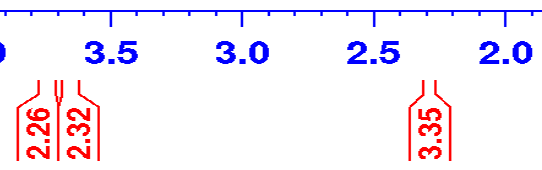

岕命 
${ }^{13} \mathrm{C}\left\{{ }^{1} \mathrm{H}\right\}$ NMR (101 MHz, $\left.\mathrm{CDCl}_{3}, 298 \mathrm{~K}\right)$
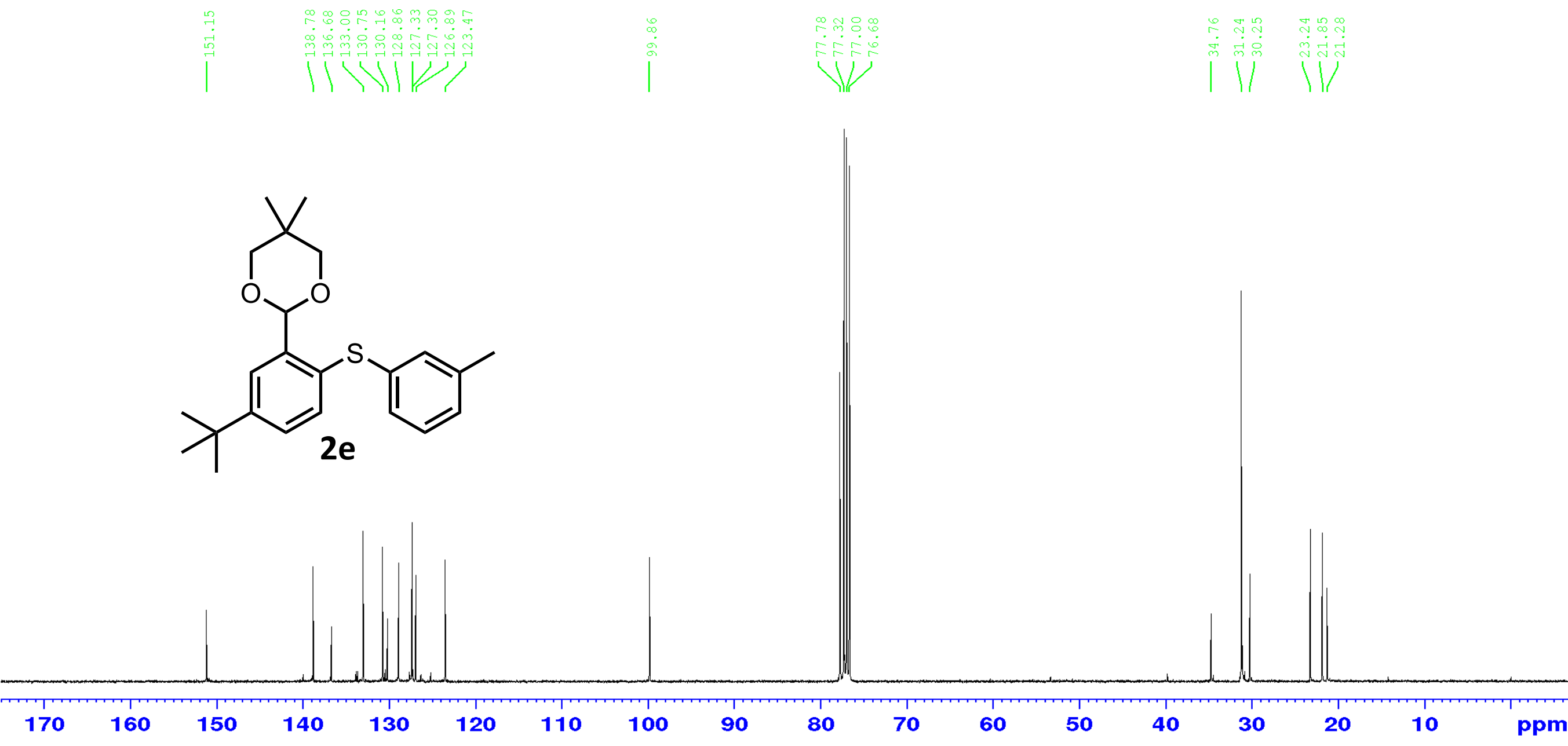
${ }^{1} \mathrm{H} \mathrm{NMR}\left(400 \mathrm{MHz}, \mathrm{CDCl}_{3}, 298 \mathrm{~K}\right)$
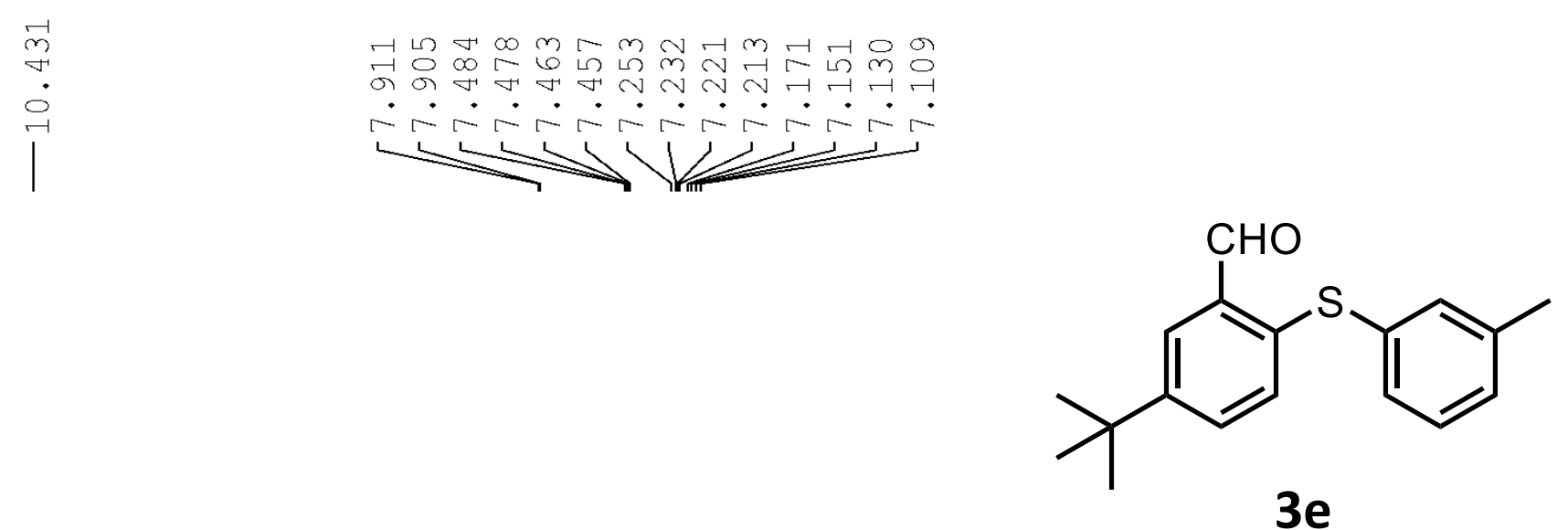

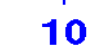

용

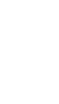

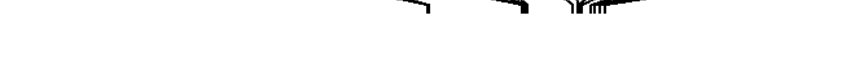

\section{3e}


${ }^{13} \mathrm{C}\left\{{ }^{1} \mathrm{H}\right\}$ NMR (101 MHz, $\left.\mathrm{CDCl}_{3}, 298 \mathrm{~K}\right)$
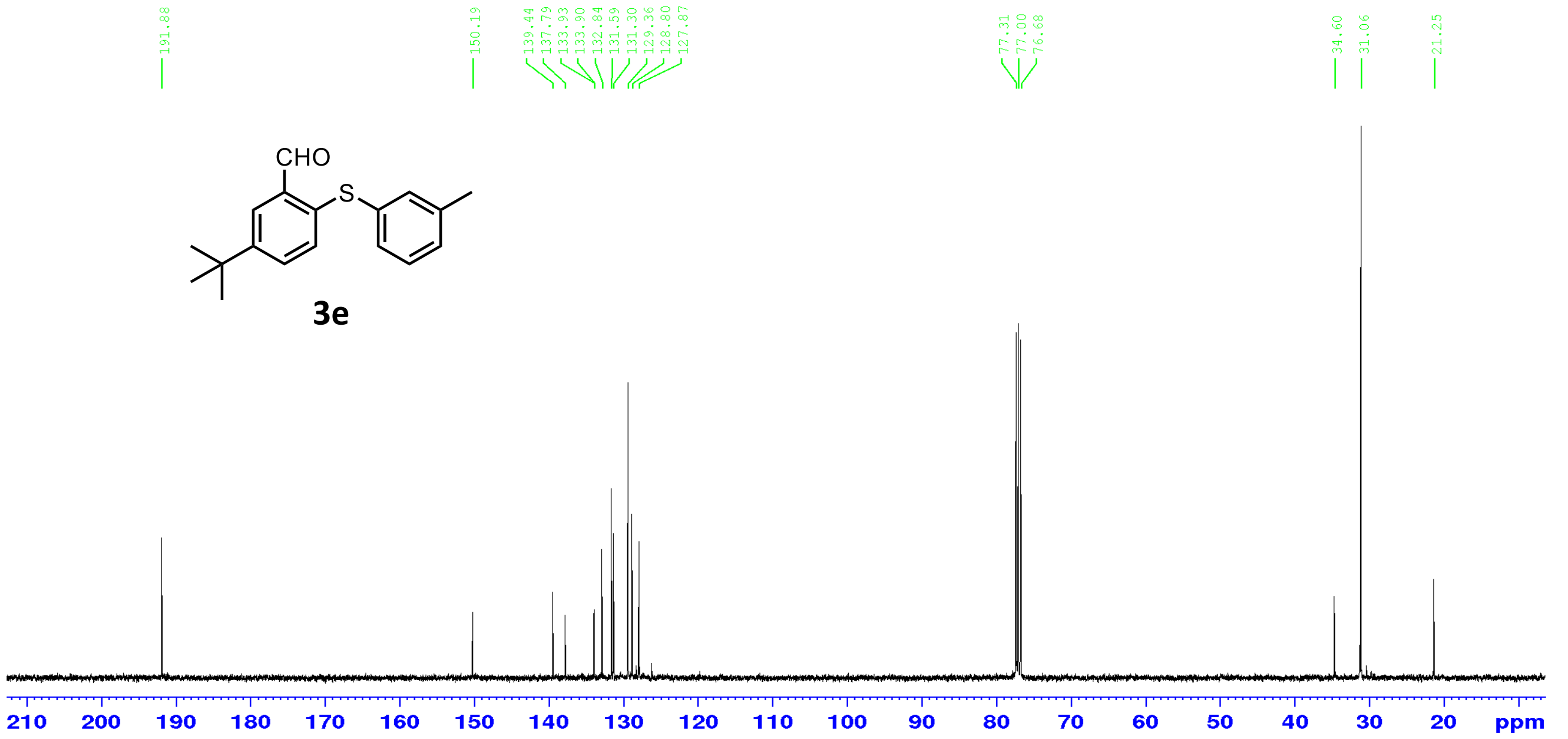
${ }^{1} \mathrm{H}$ NMR (400 MHz, $\left.\mathrm{CD}_{3} \mathrm{CN}, 298 \mathrm{~K}\right)$

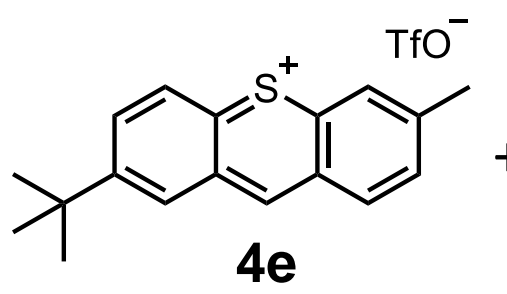

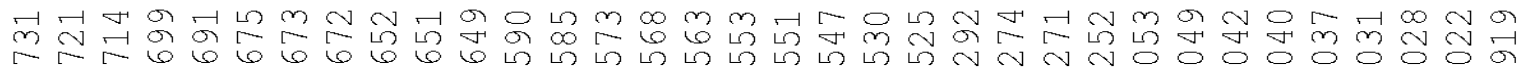

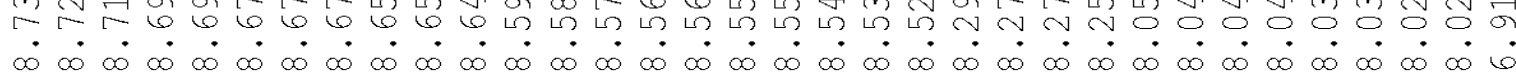

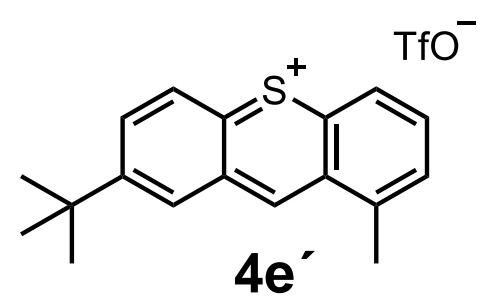

๖ • • ? ? ? ? 1

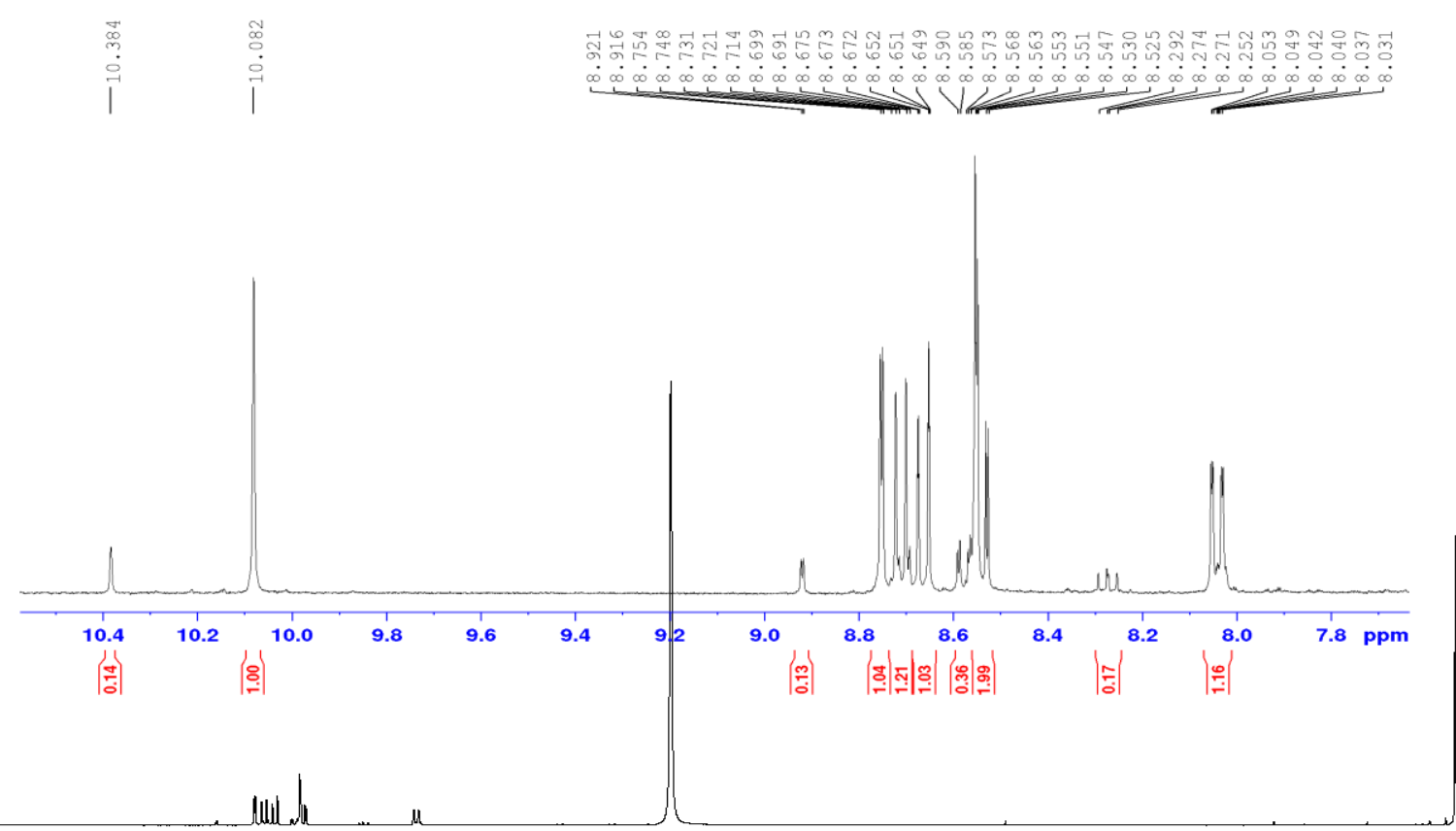

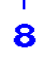

8

7
5

突 㑒

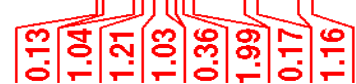

$10 \quad 9$

6

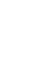

4

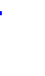


${ }^{13} \mathrm{C}\left\{{ }^{1} \mathrm{H}\right\}$ NMR (101 MHz, $\left.\mathrm{CD}_{3} \mathrm{CN}, 298 \mathrm{~K}\right)$

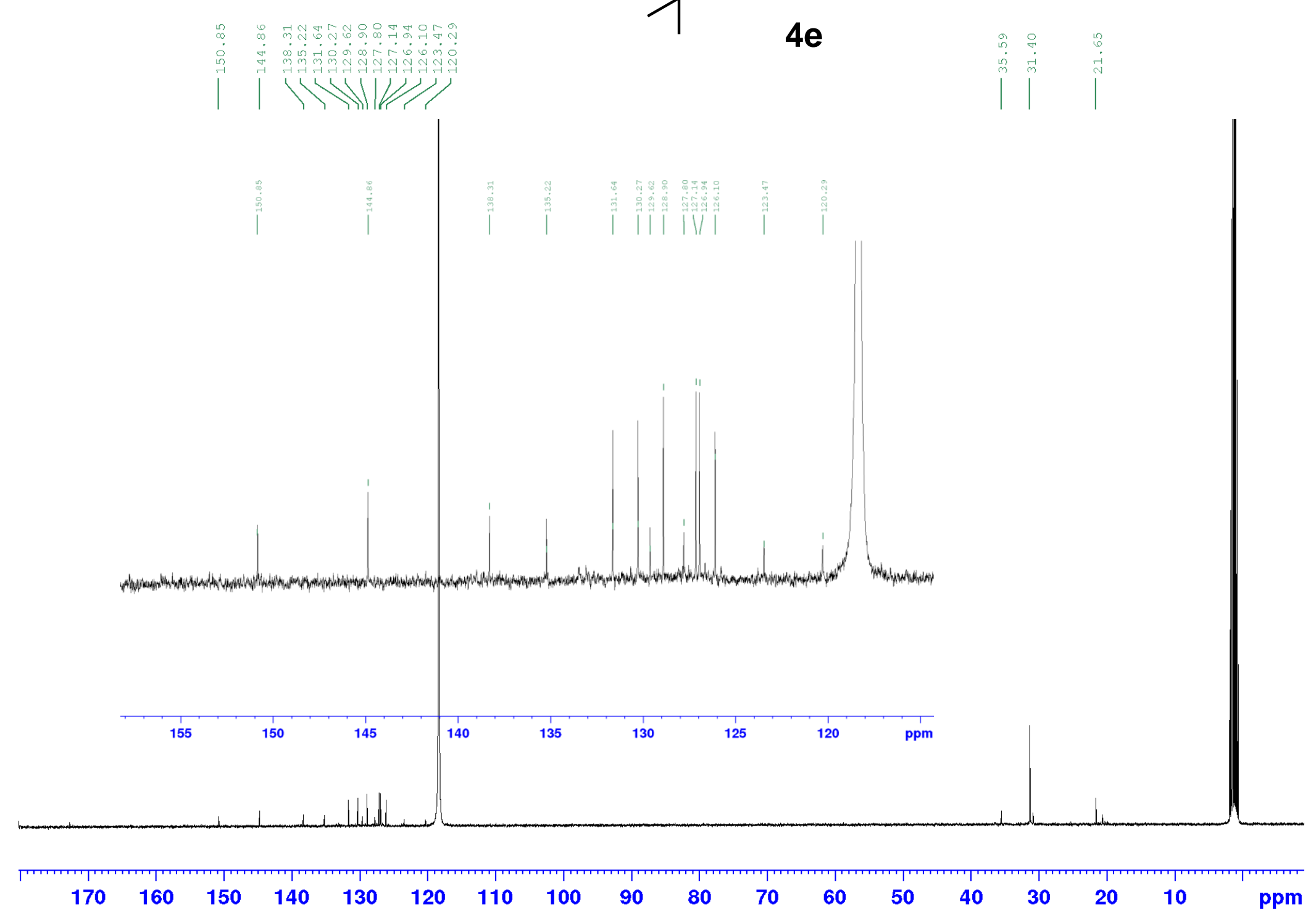


${ }^{1} \mathrm{H}$ NMR (400 MHz, $\mathrm{CDCl}_{3}, 298 \mathrm{~K}$ )
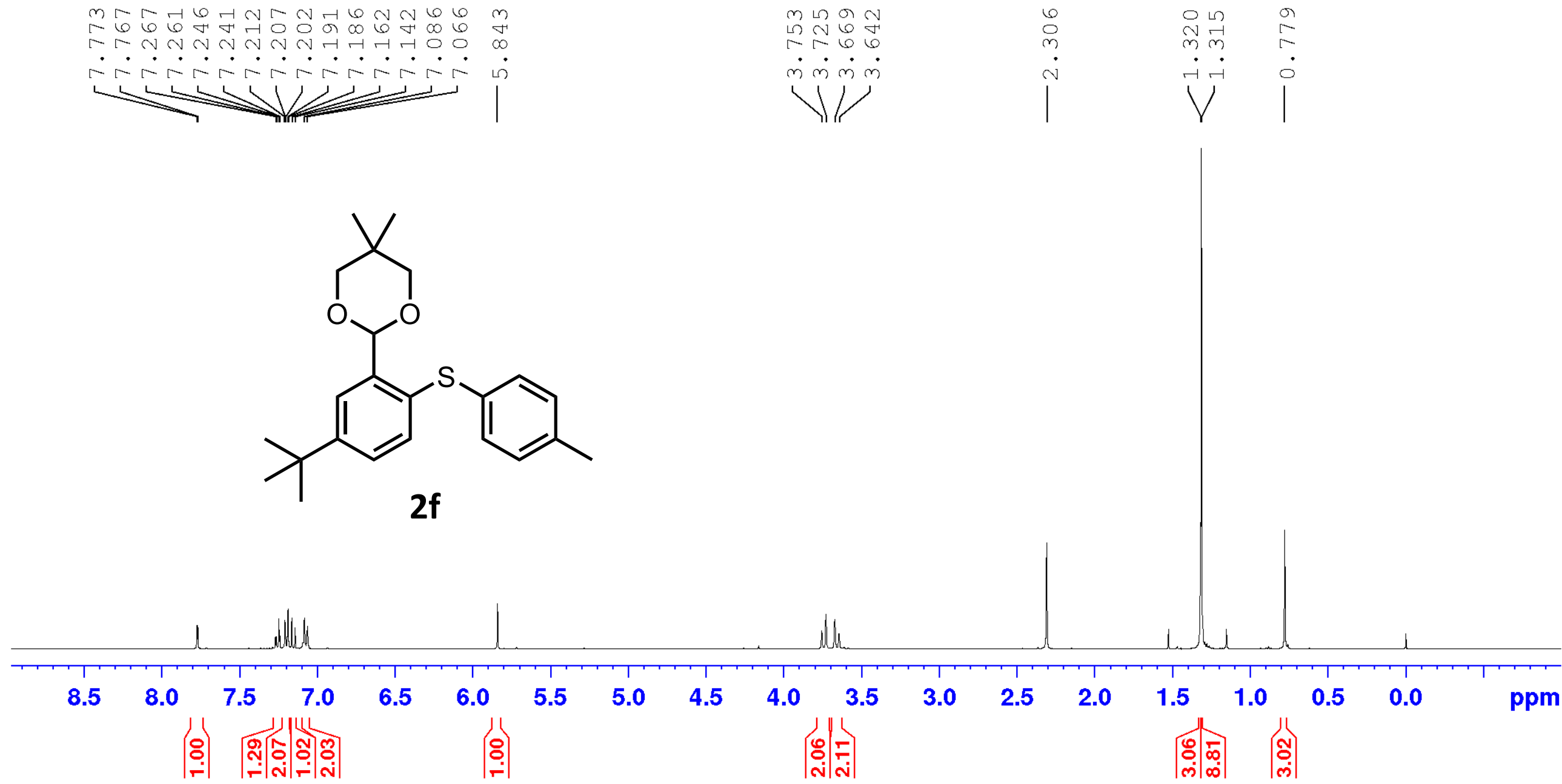
${ }^{13} \mathrm{C}\left\{{ }^{1} \mathrm{H}\right\}$ NMR (101 MHz, $\left.\mathrm{CDCl}_{3}, 298 \mathrm{~K}\right)$

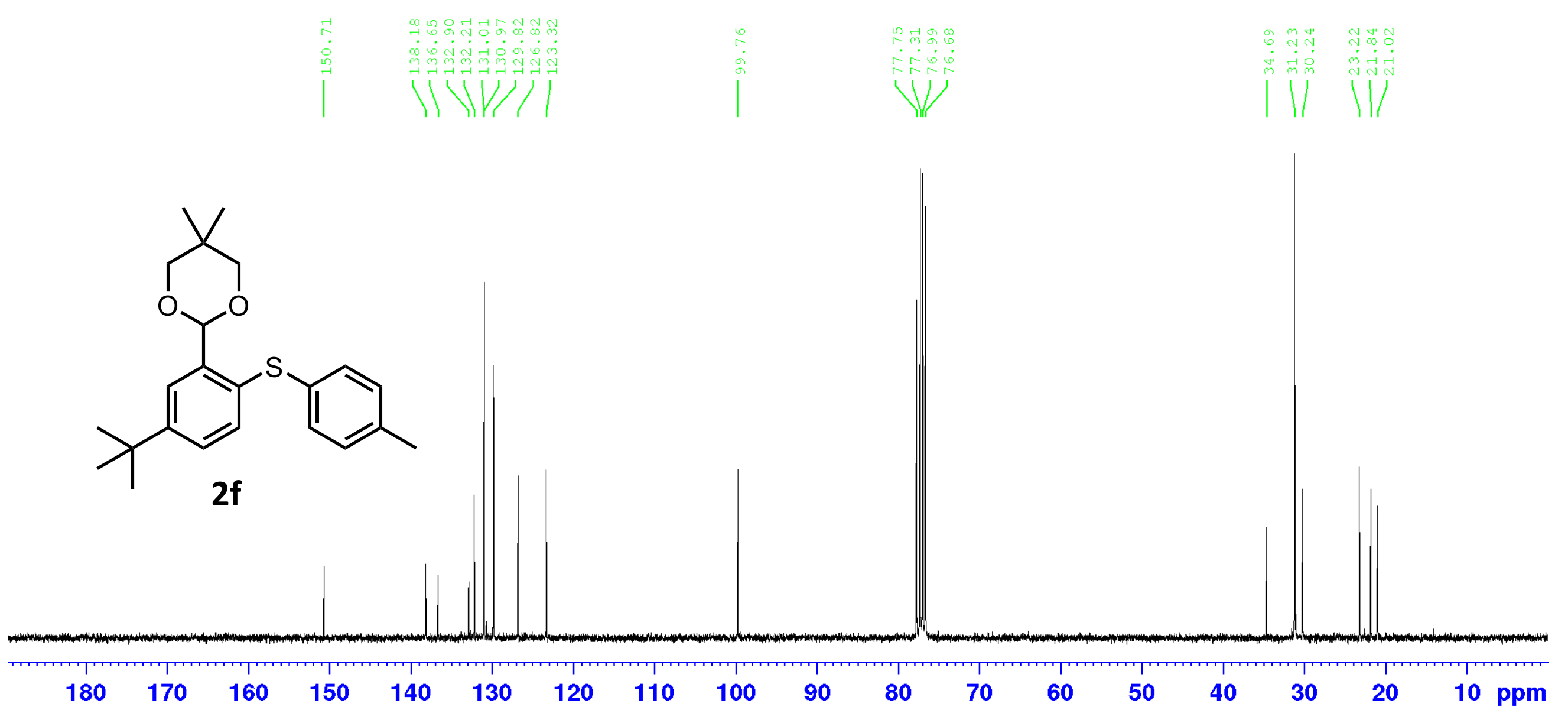


${ }^{1} \mathrm{H}$ NMR (400 MHz, $\left.\mathrm{CDCl}_{3}, 298 \mathrm{~K}\right)$
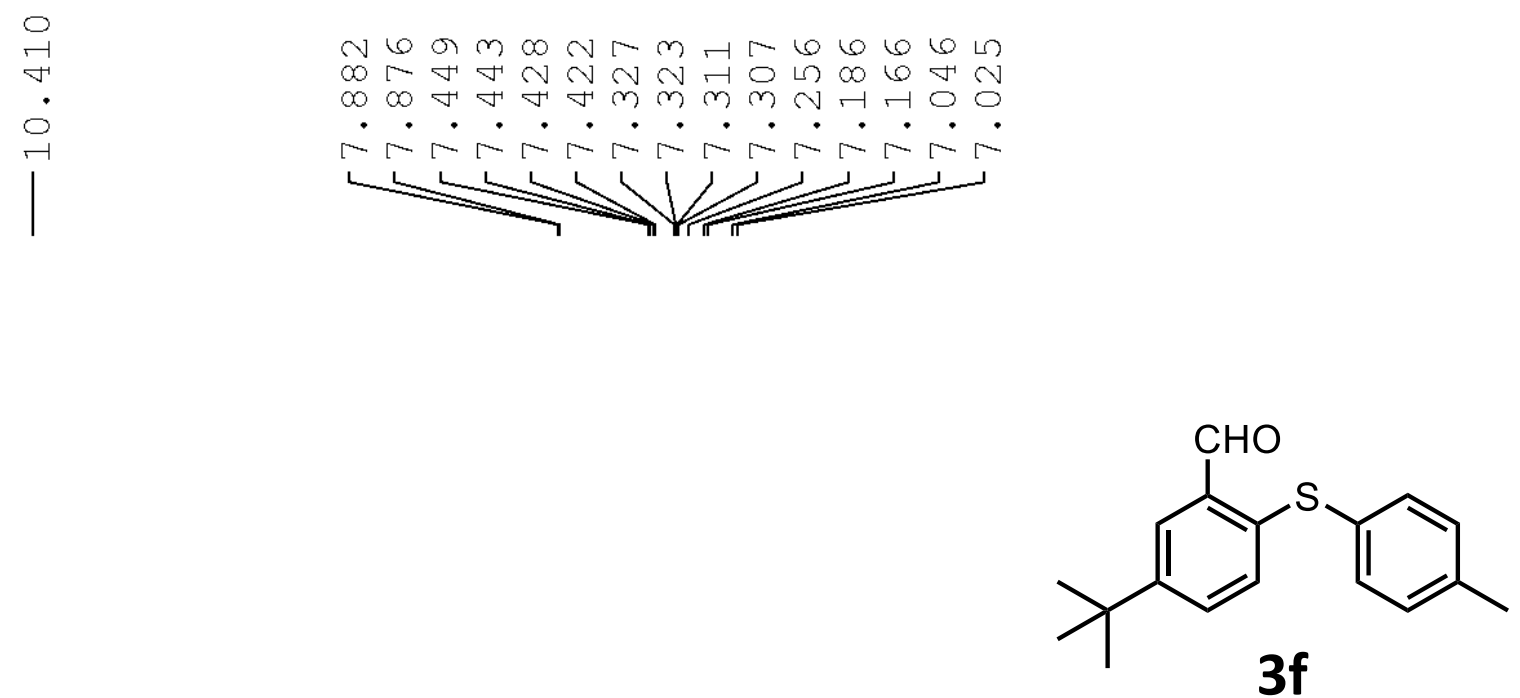

\section{9}

이미

| 
${ }^{13} \mathrm{C}\left\{{ }^{1} \mathrm{H}\right\}$ NMR (101 MHz, $\left.\mathrm{CDCl}_{3}, 298 \mathrm{~K}\right)$
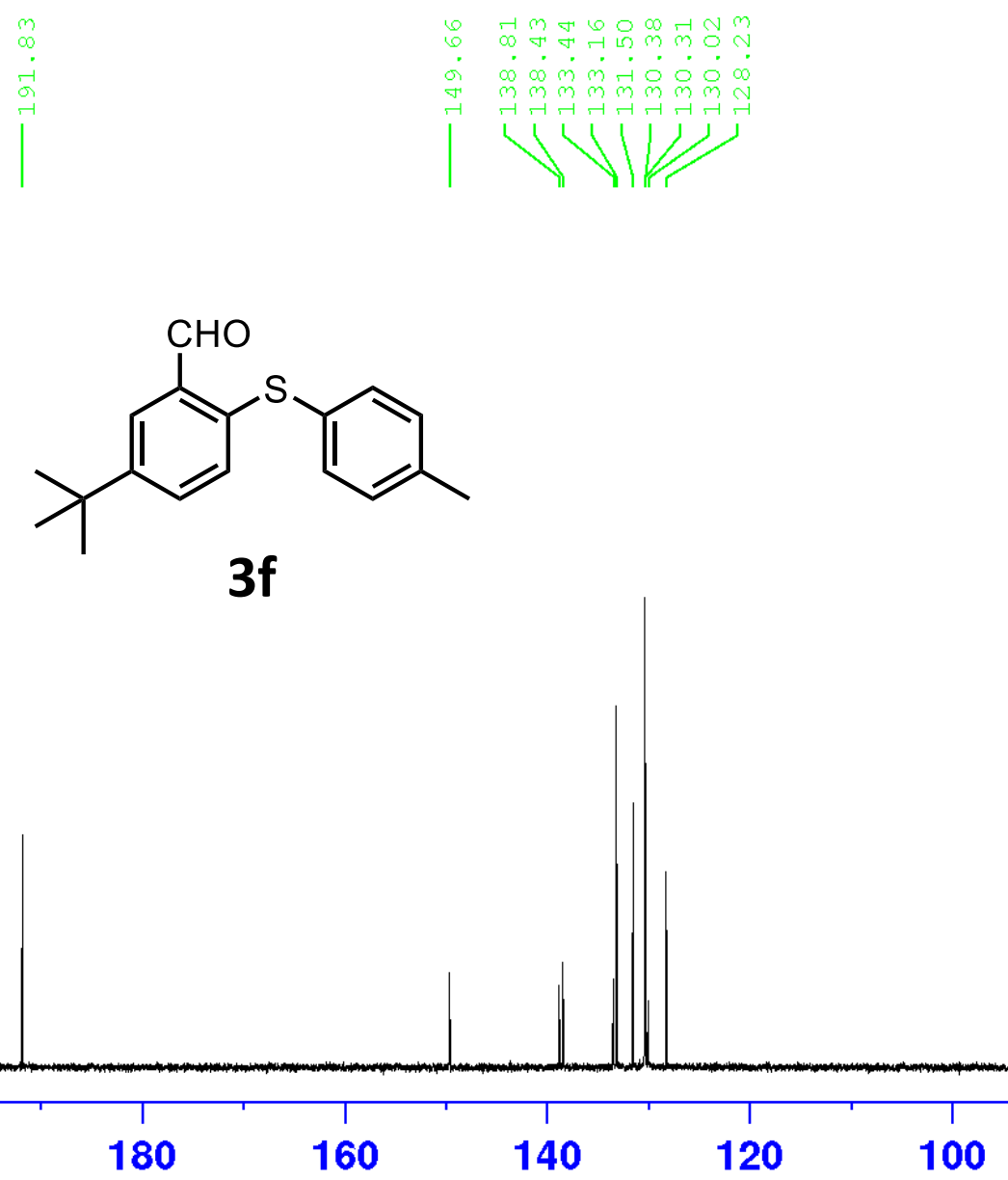
(2) 
${ }^{1} \mathrm{H}$ NMR (400 MHz, CD $\left.{ }_{3} \mathrm{CN}, 298 \mathrm{~K}\right)$
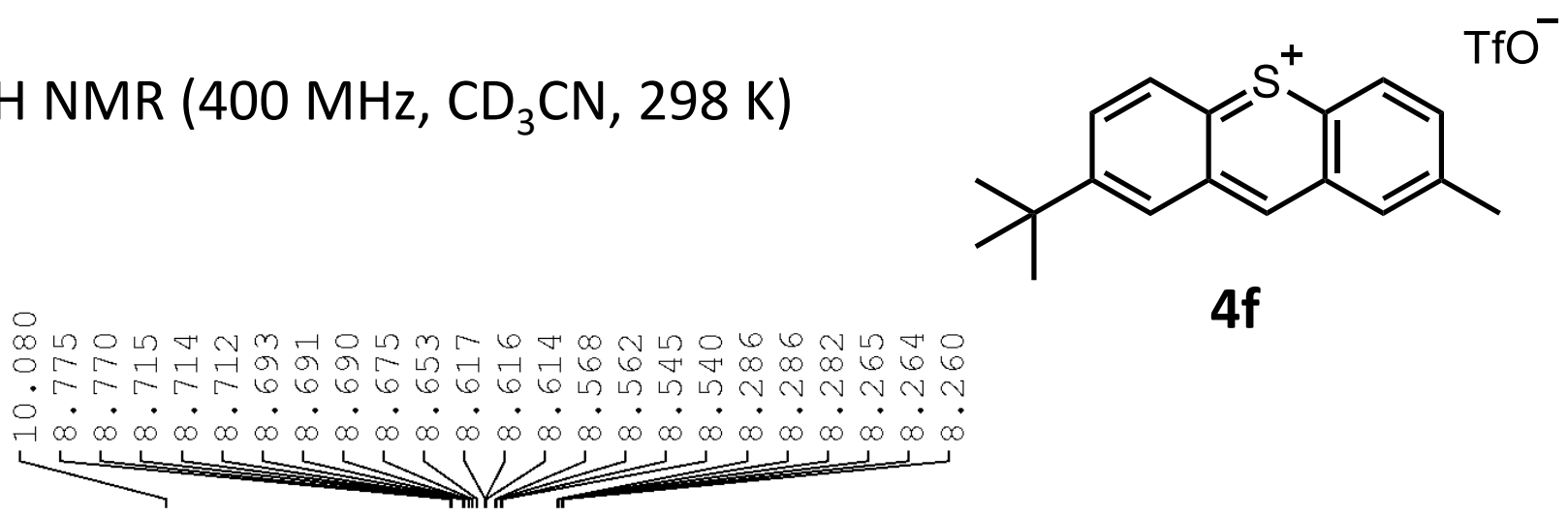

$4 f$

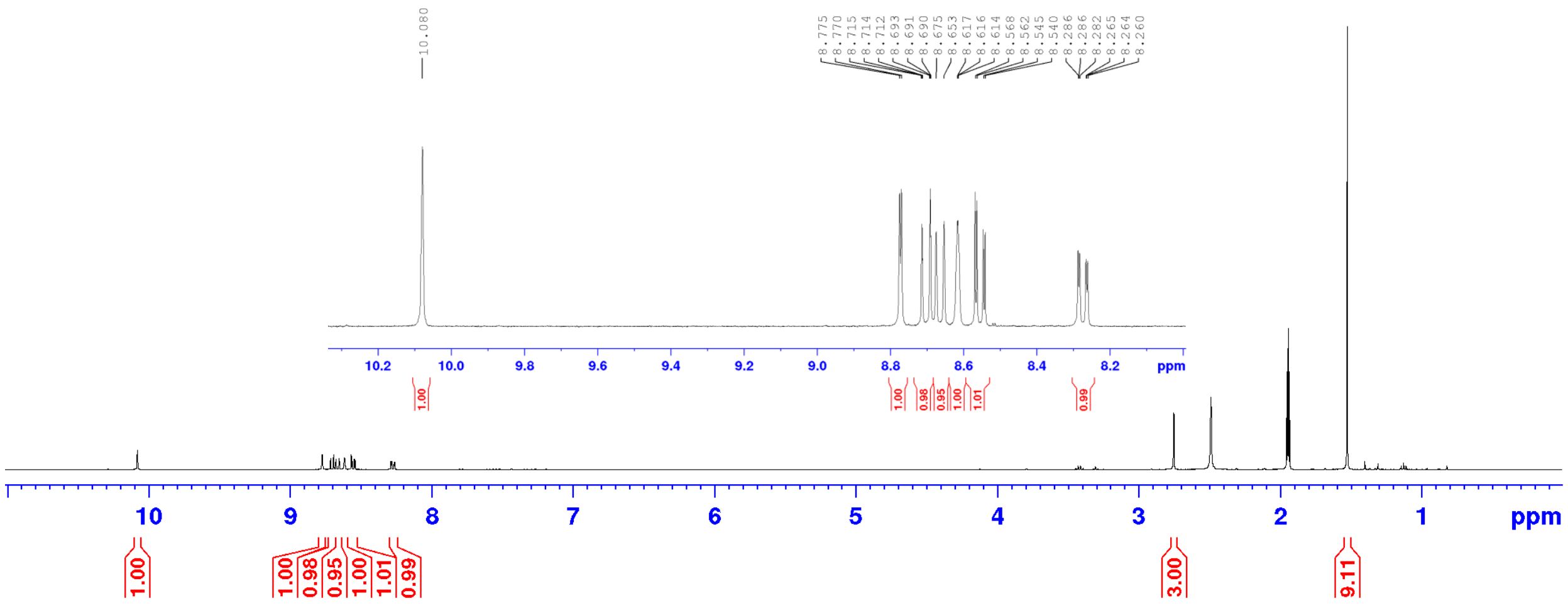


${ }^{13} \mathrm{C}\left\{{ }^{1} \mathrm{H}\right\} \mathrm{NMR}\left(101 \mathrm{MHz}, \mathrm{CD}_{3} \mathrm{CN}, 298 \mathrm{~K}\right)$

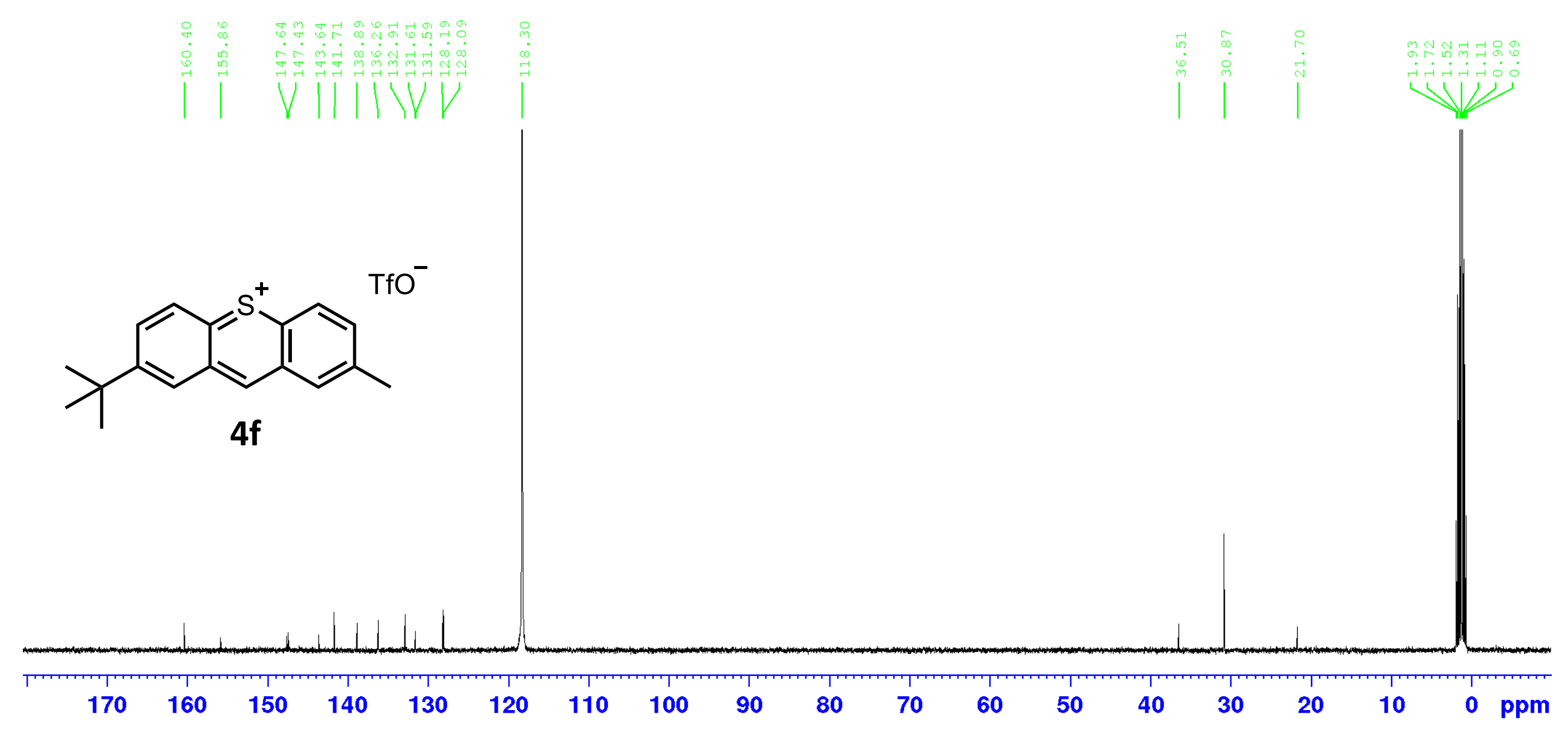


${ }^{1} \mathrm{H} \mathrm{NMR}\left(400 \mathrm{MHz}, \mathrm{CDCl}_{3}, 298 \mathrm{~K}\right)$
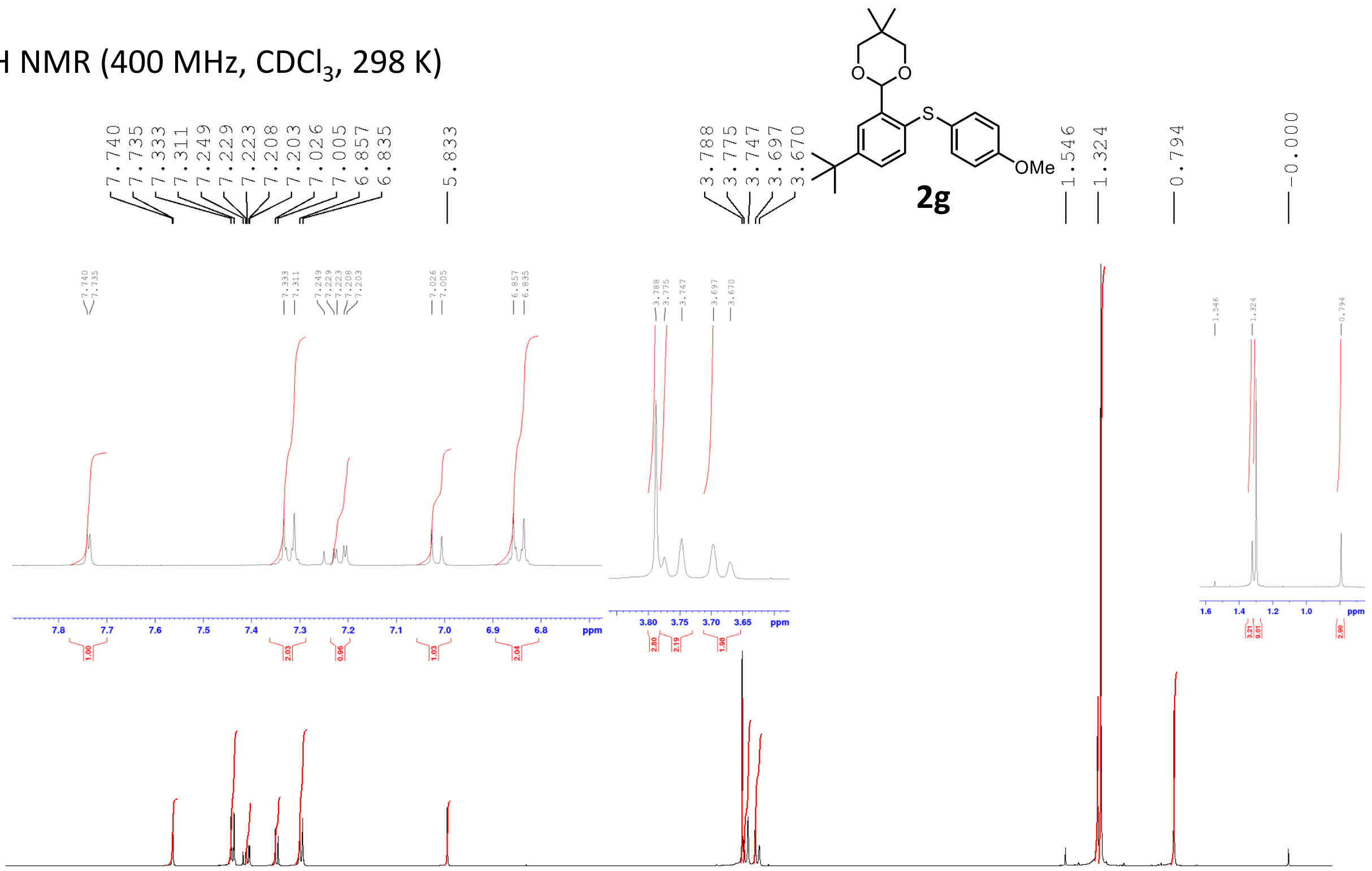

\begin{tabular}{|c|c|c|c|c|c|c|c|c|c|c|c|c|c|c|c|c|c|}
\hline 8.5 & 8.0 & $\begin{array}{l}7.5 \\
\end{array}$ & 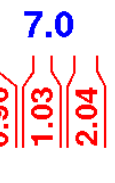 & 6.5 & 6.0 & 5.5 & 5.0 & 4.5 & $\begin{array}{r}4.0 \\
\end{array}$ & | & 3.0 & 2.5 & 2.0 & & 1.0 & 0.5 & ppm \\
\hline
\end{tabular}


${ }^{13} \mathrm{C}$ NMR (101 MHz, CDCl, $298 \mathrm{~K}$ )
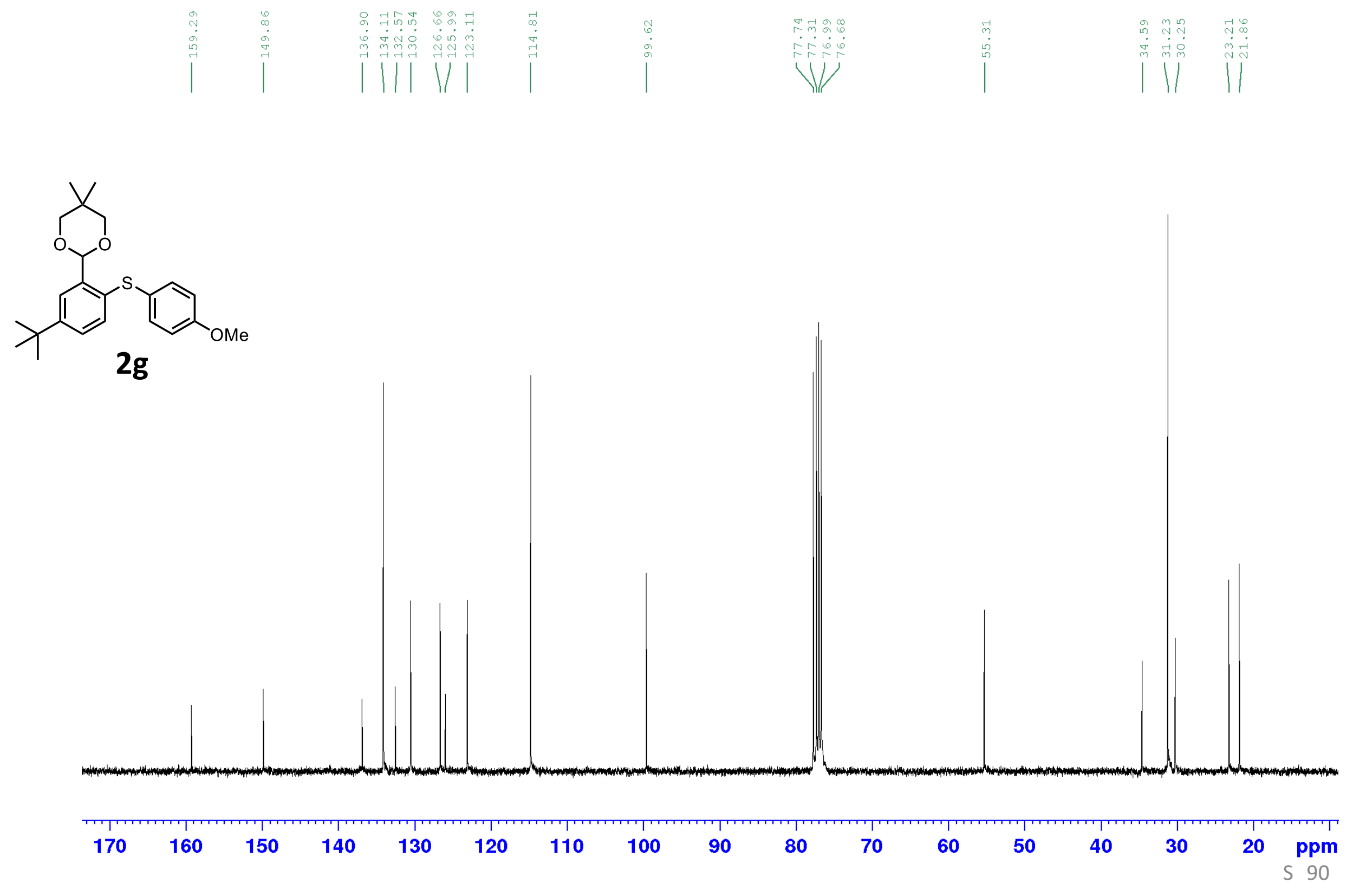
${ }^{1} \mathrm{H}$ NMR (400 MHz, $\mathrm{CDCl}_{3}, 298 \mathrm{~K}$ )
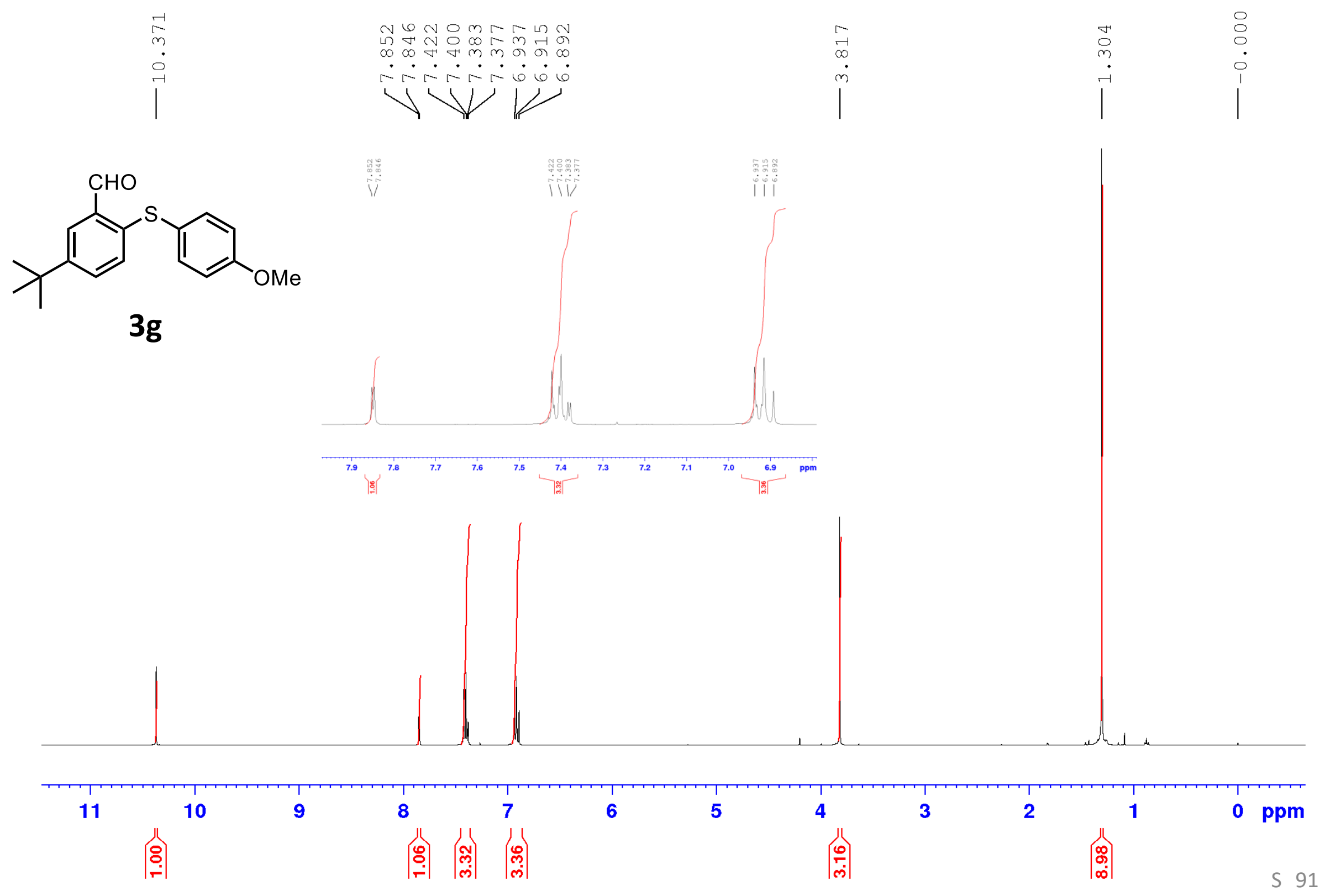
${ }^{13} \mathrm{C}$ NMR (101 MHz, CDCl 3 , $298 \mathrm{~K}$ )
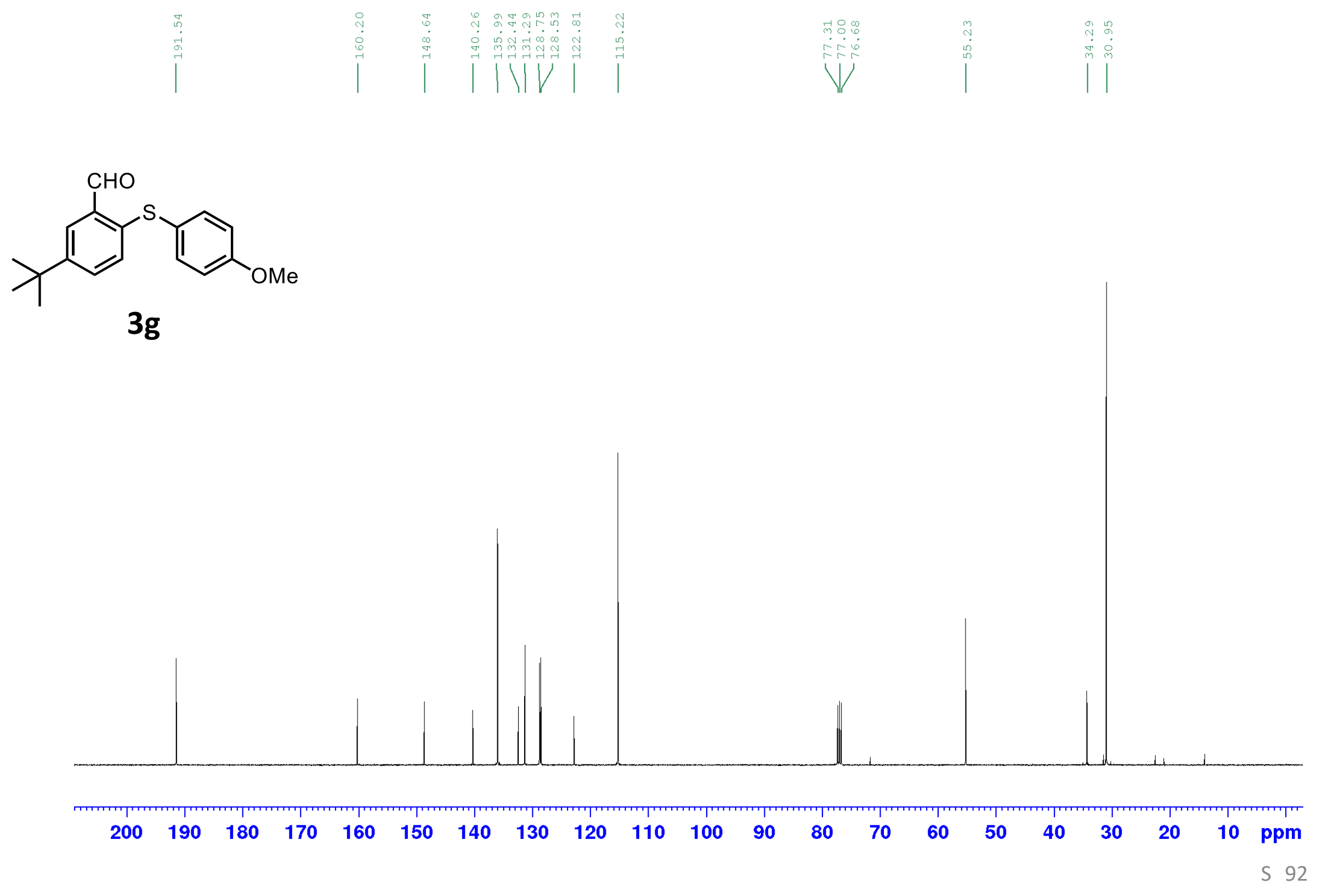
${ }^{1} \mathrm{H}$ NMR (400 MHz, CD 3 CN, $298 \mathrm{~K}$ )

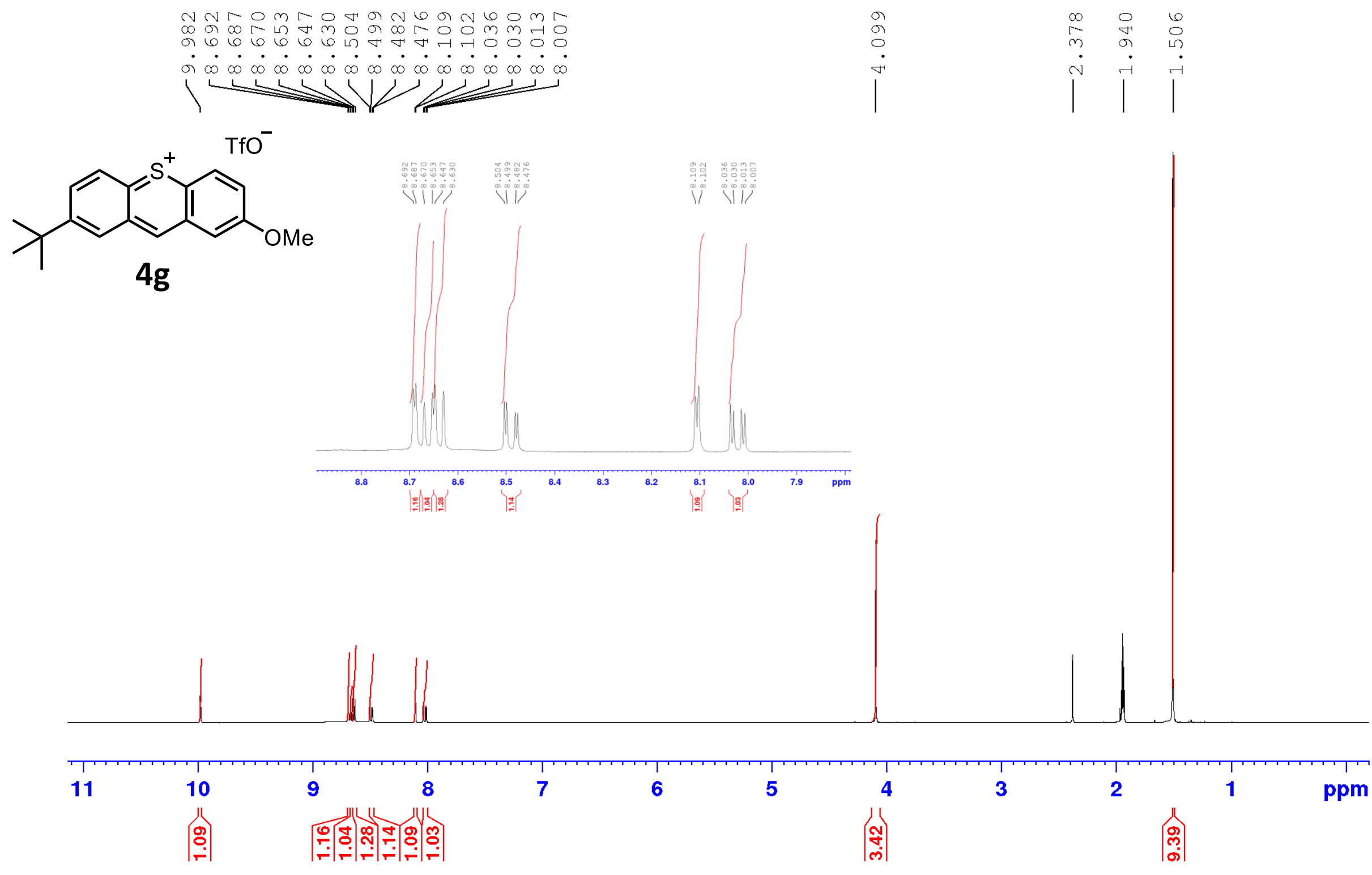


${ }^{13} \mathrm{C}$ NMR (101 MHz, CD 3 CN, $298 \mathrm{~K}$ )
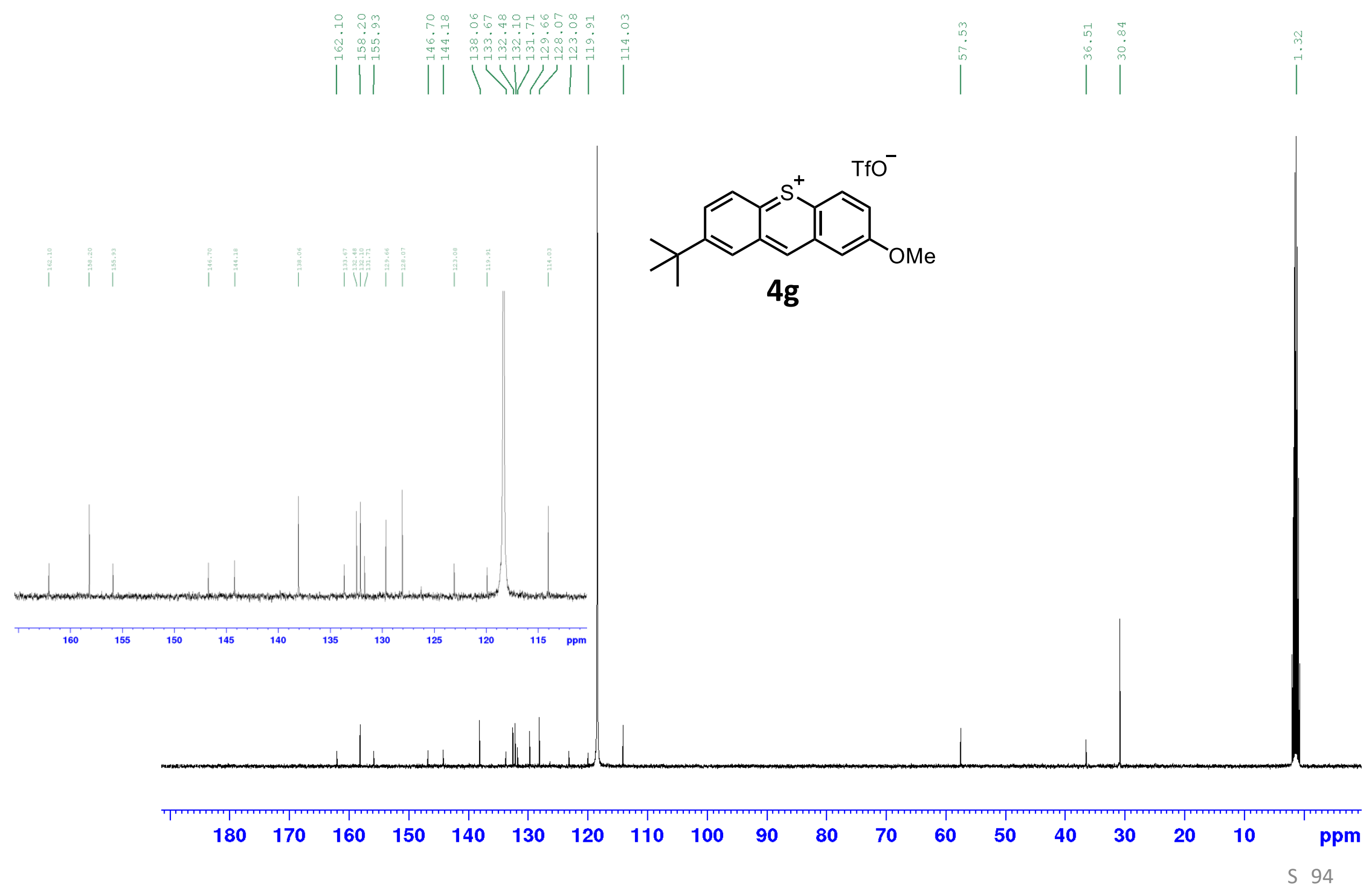
${ }^{1} \mathrm{H} \mathrm{NMR} \mathrm{(400} \mathrm{MHz,} \mathrm{CDCl}_{3}, 298 \mathrm{~K}$ )

ம

N

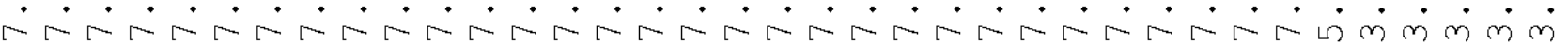

LbL blo

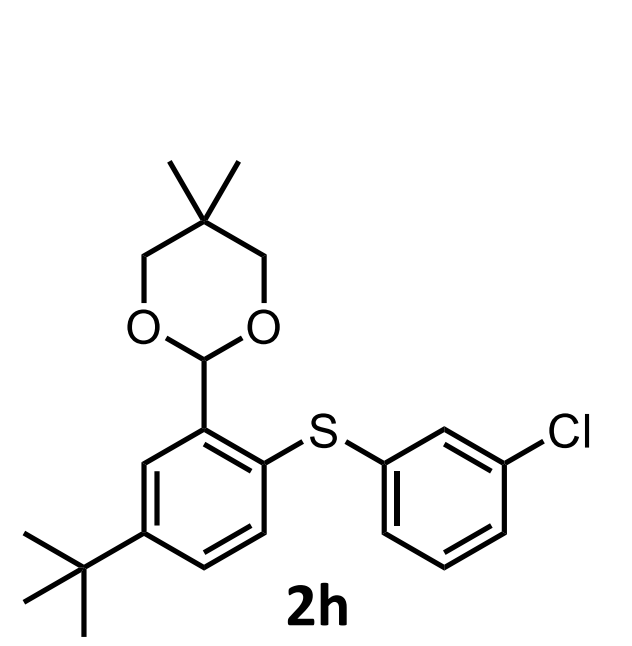

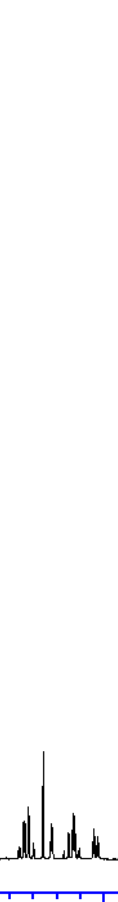

8
|

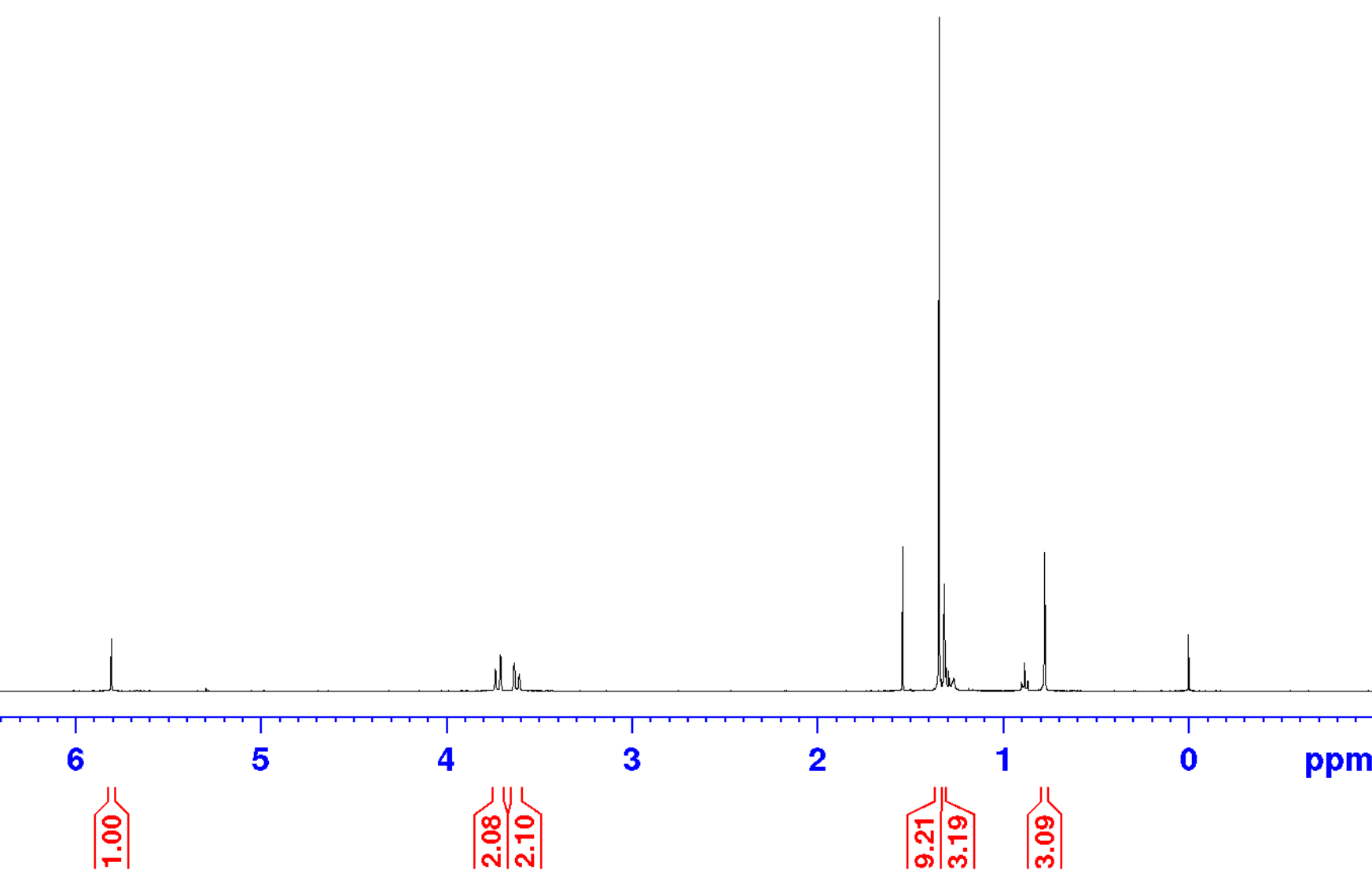


${ }^{13} \mathrm{C}\left\{{ }^{1} \mathrm{H}\right\}$ NMR (101 MHz, $\left.\mathrm{CDCl}_{3}, 298 \mathrm{~K}\right)$
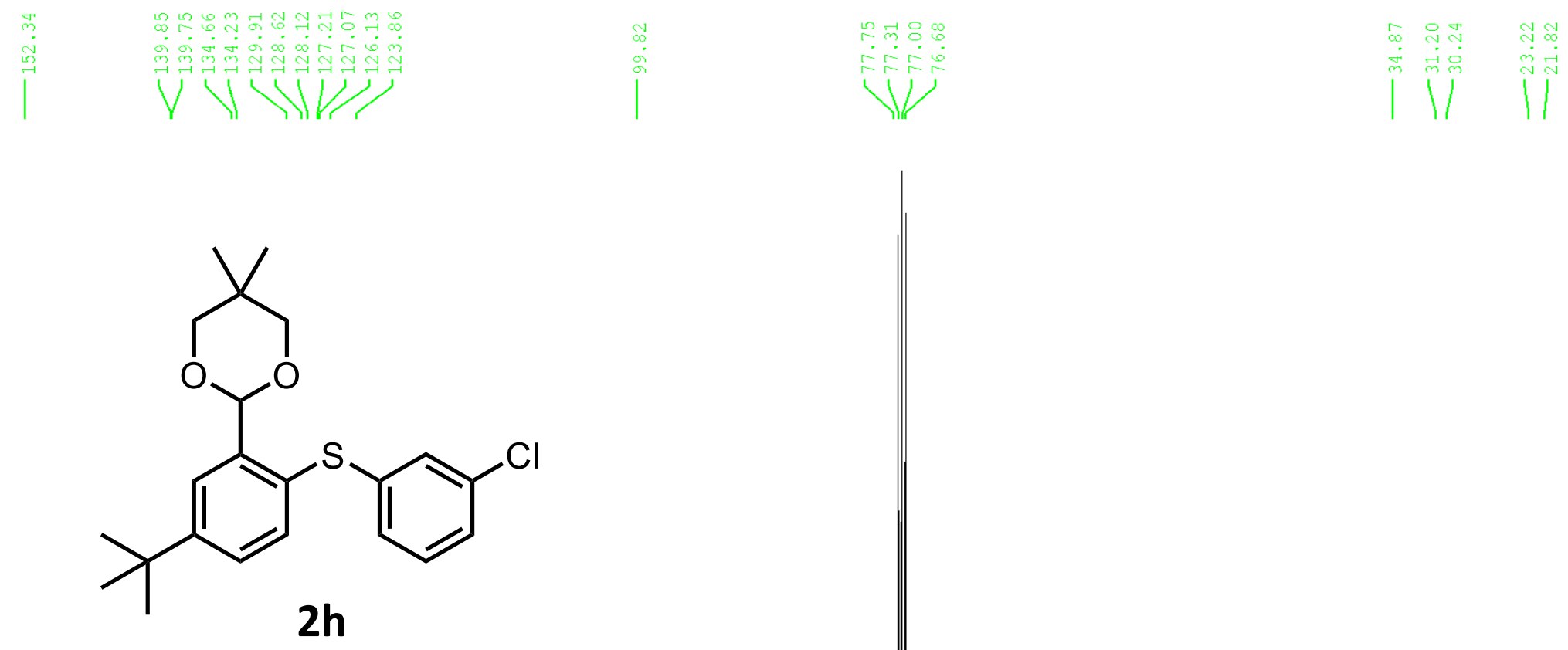
${ }^{1} \mathrm{H} \mathrm{NMR}\left(400 \mathrm{MHz}, \mathrm{CDCl}_{3}, 298 \mathrm{~K}\right.$ )
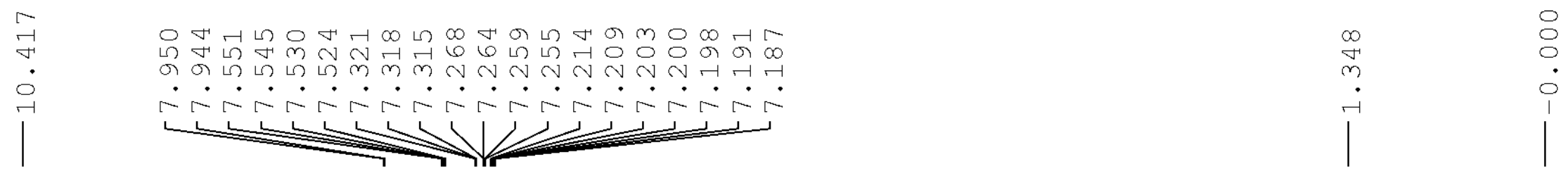<smiles>CC(C)(C)c1ccc(Sc2cccc(Cl)c2)c(C=O)c1</smiles>
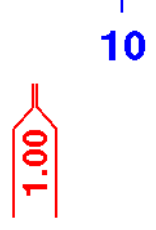

(10)

우- 
${ }^{13} \mathrm{C}\left\{{ }^{1} \mathrm{H}\right\}$ NMR (101 MHz, $\left.\mathrm{CDCl}_{3}, 298 \mathrm{~K}\right)$
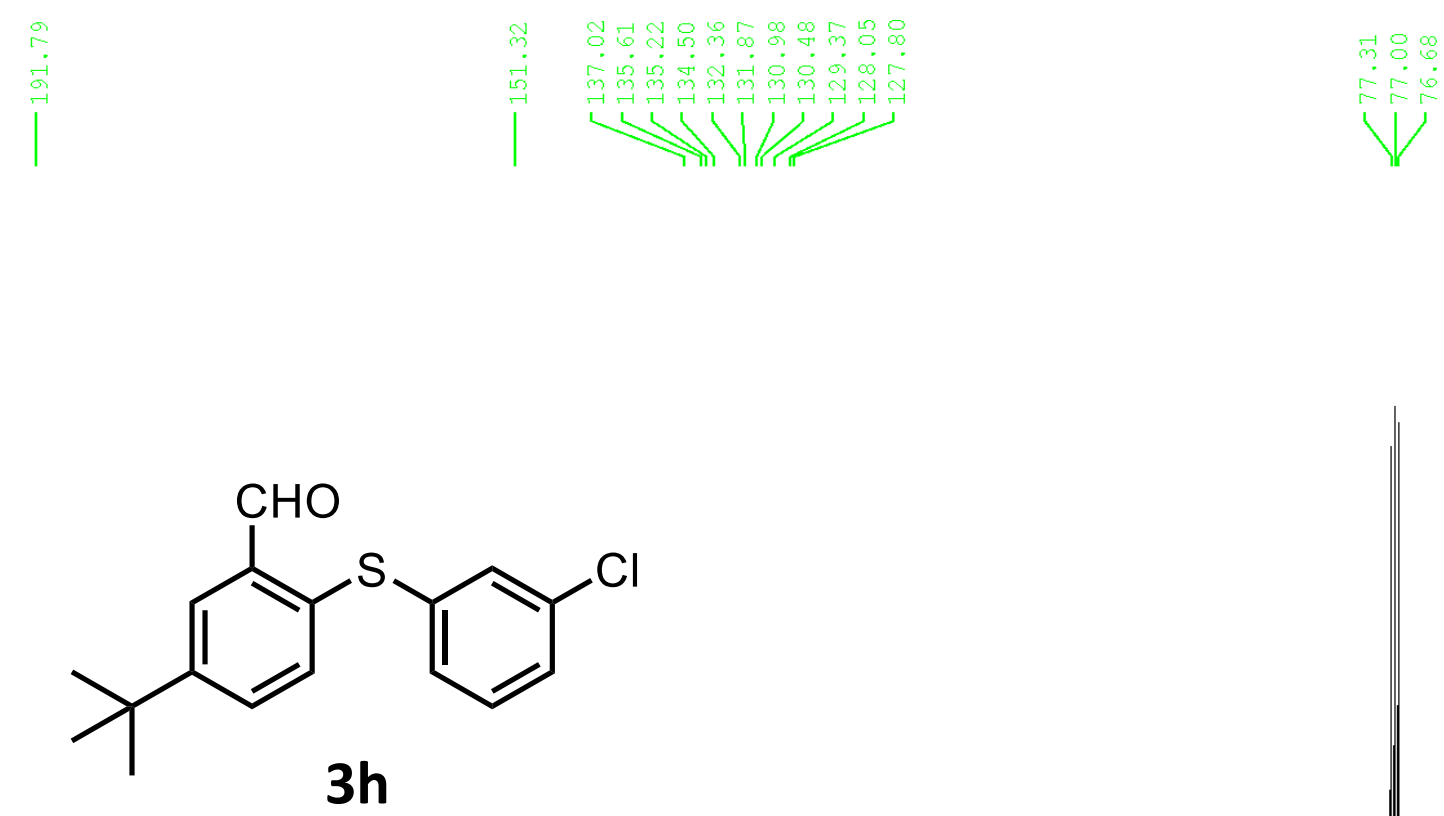
${ }^{1} \mathrm{H}$ NMR (400 MHz, CD 3 CN, $\left.298 \mathrm{~K}\right)$
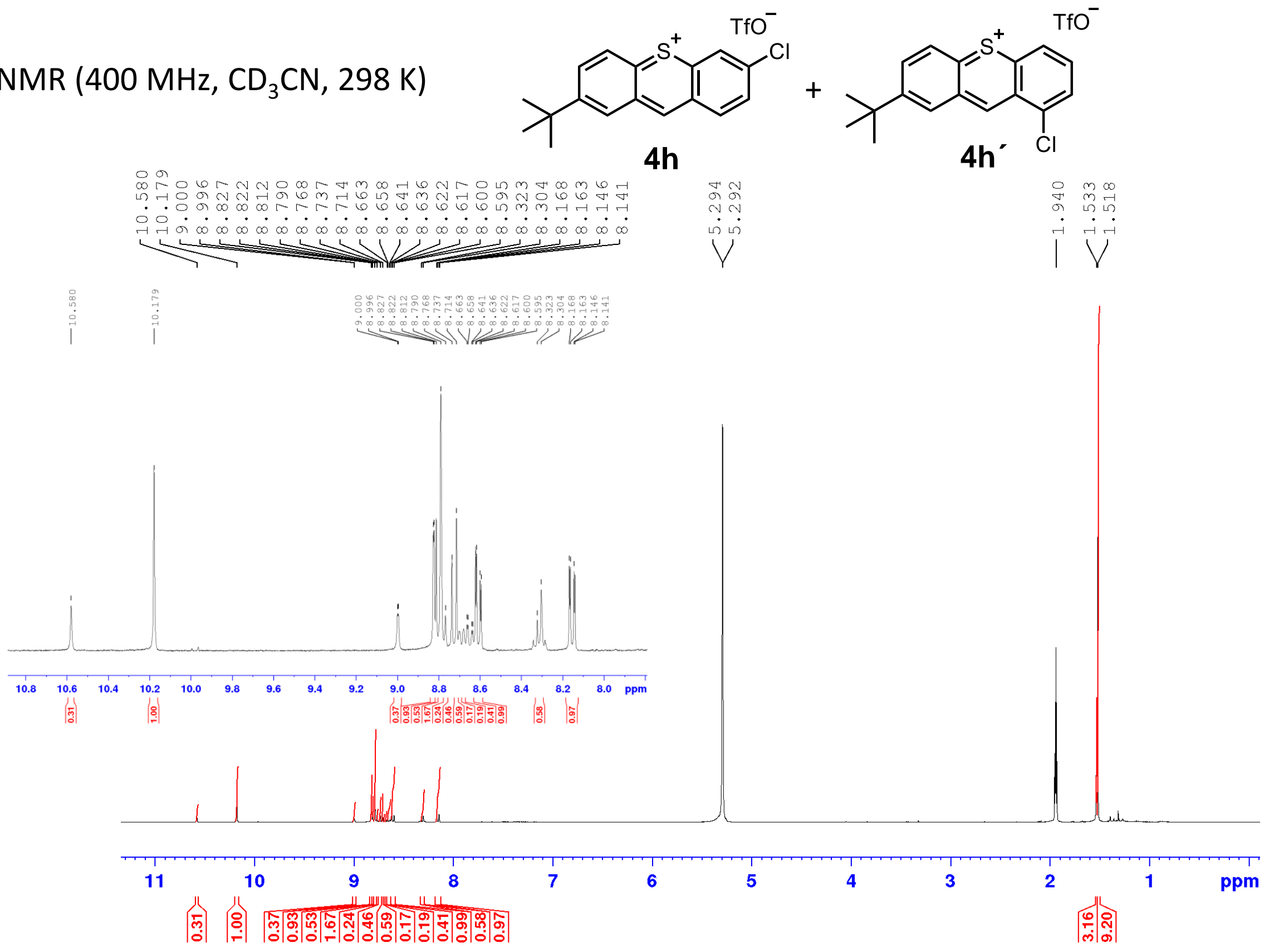
${ }^{13} \mathrm{C}\left\{{ }^{1} \mathrm{H}\right\}$ NMR (101 MHz, CD $\left.3 \mathrm{CN}, 298 \mathrm{~K}\right)$

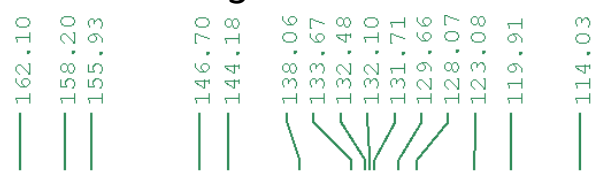

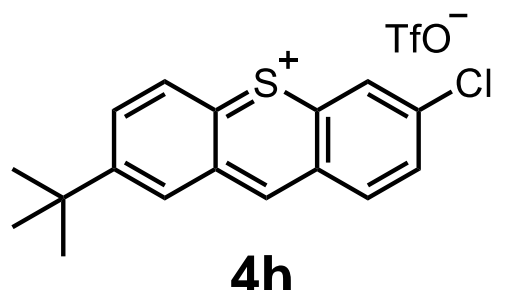

4h

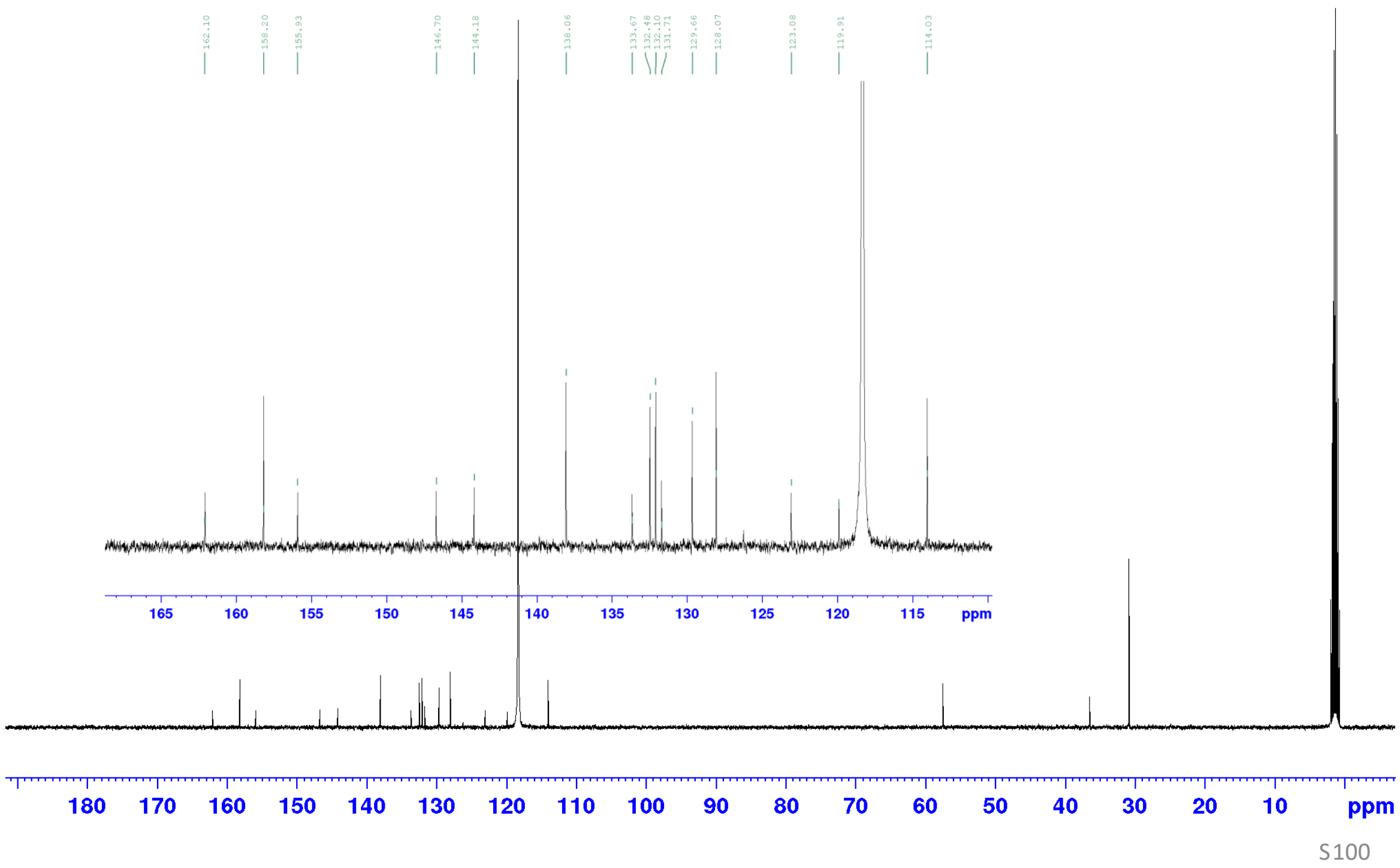


${ }^{1} \mathrm{H} \mathrm{NMR}\left(400 \mathrm{MHz}, \mathrm{CDCl}_{3}, 298 \mathrm{~K}\right.$ )
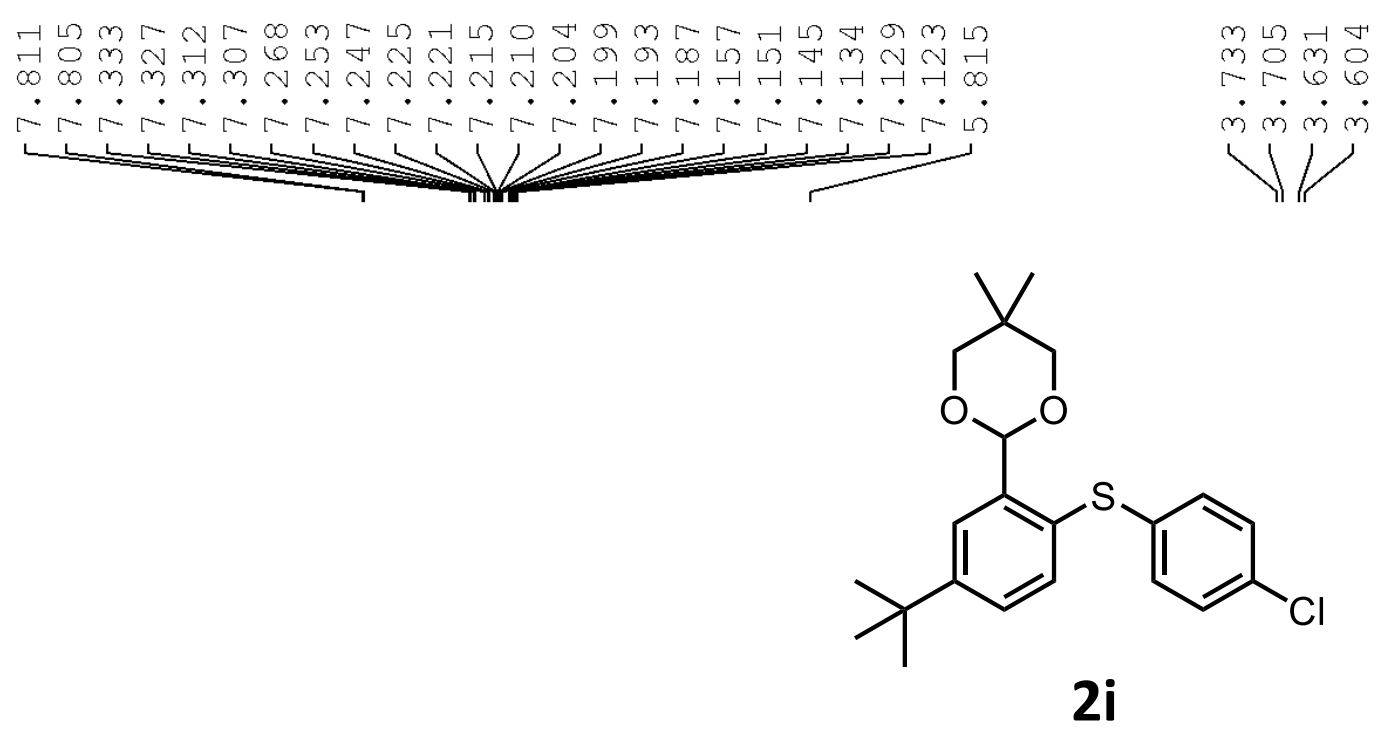

$2 \mathbf{i}$

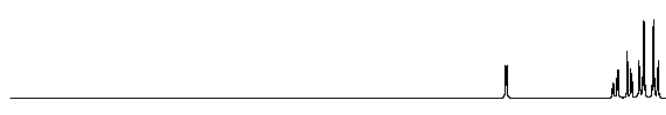

9

8

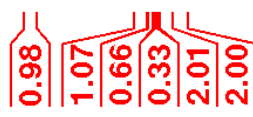

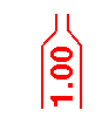

5

,

|

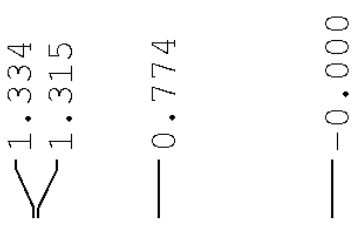
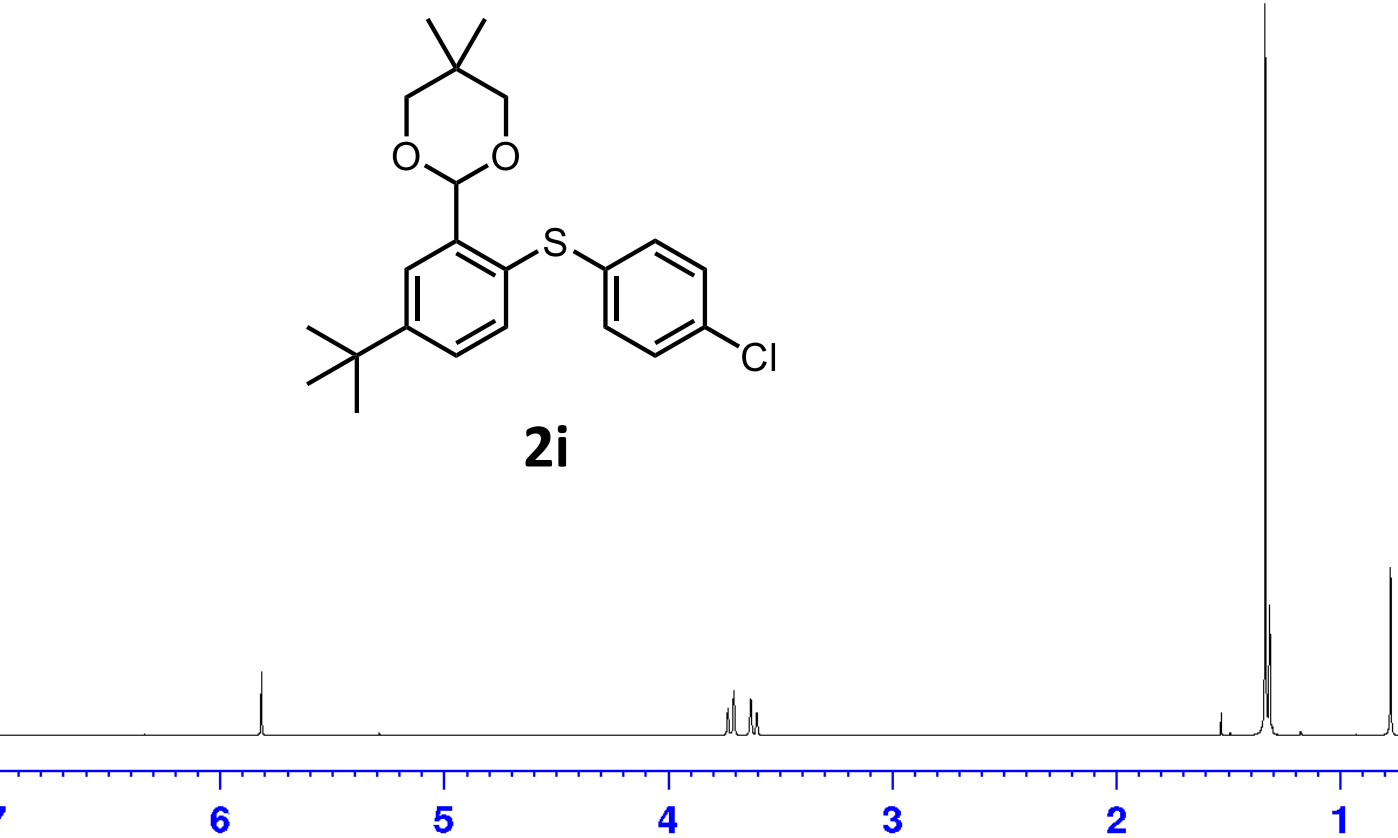

3

2

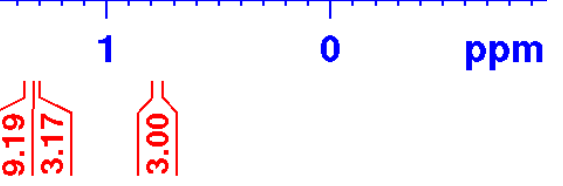


${ }^{13} \mathrm{C}\left\{{ }^{1} \mathrm{H}\right\}$ NMR (101 MHz, $\left.\mathrm{CDCl}_{3}, 298 \mathrm{~K}\right)$

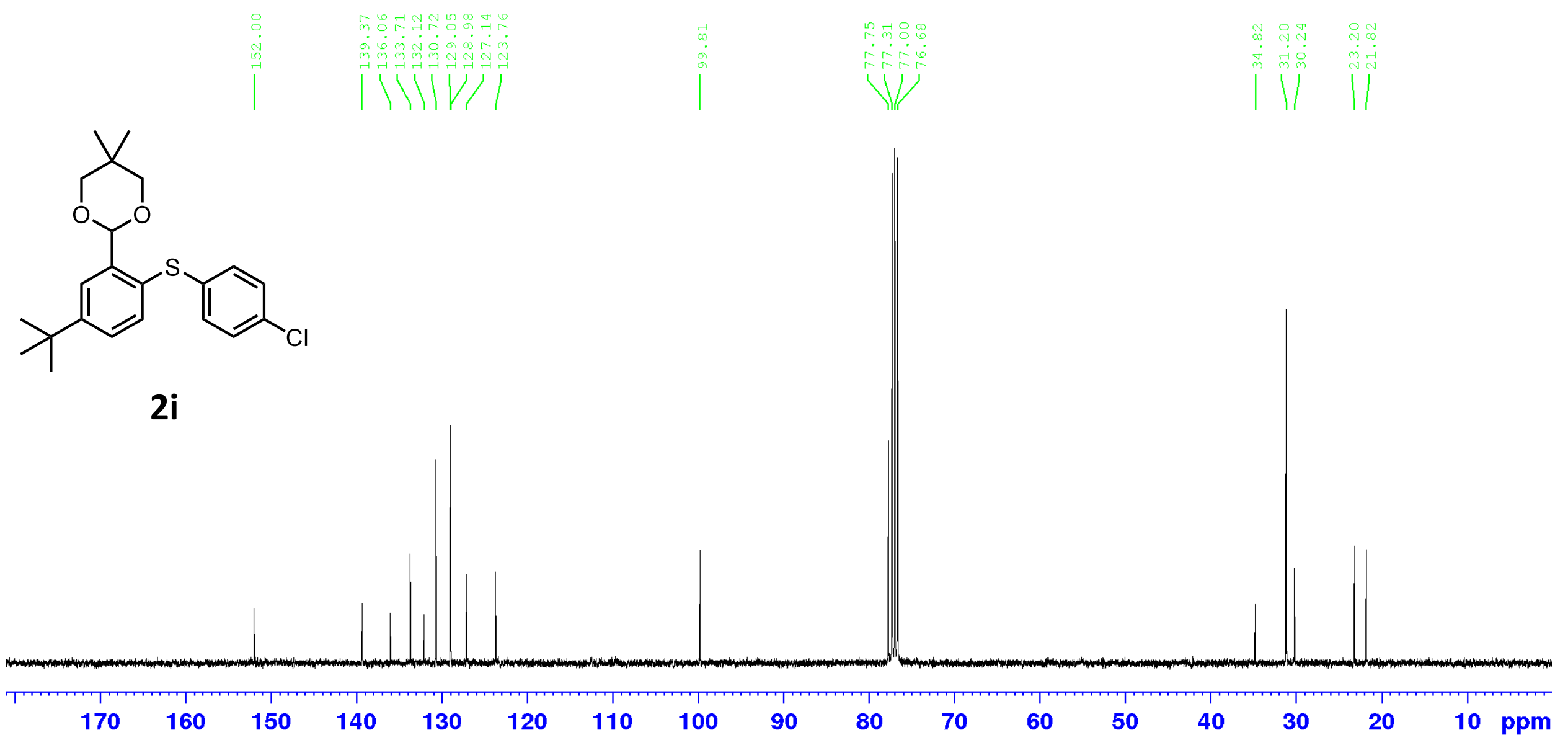


${ }^{1} \mathrm{H} \mathrm{NMR}\left(400 \mathrm{MHz}, \mathrm{CDCl}_{3}, 298 \mathrm{~K}\right.$ )

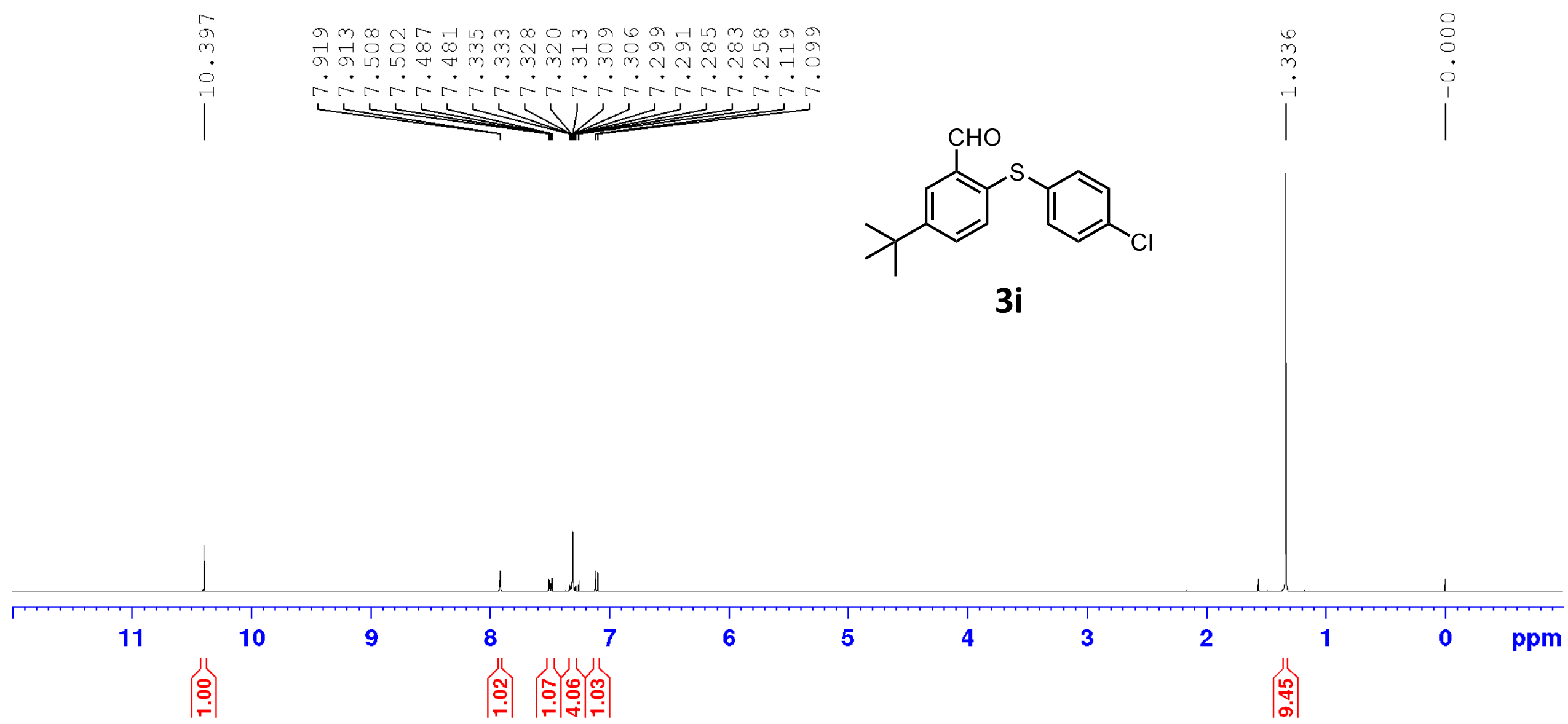


${ }^{13} \mathrm{C}\left\{{ }^{1} \mathrm{H}\right\}$ NMR (101 MHz, $\left.\mathrm{CDCl}_{3}, 298 \mathrm{~K}\right)$

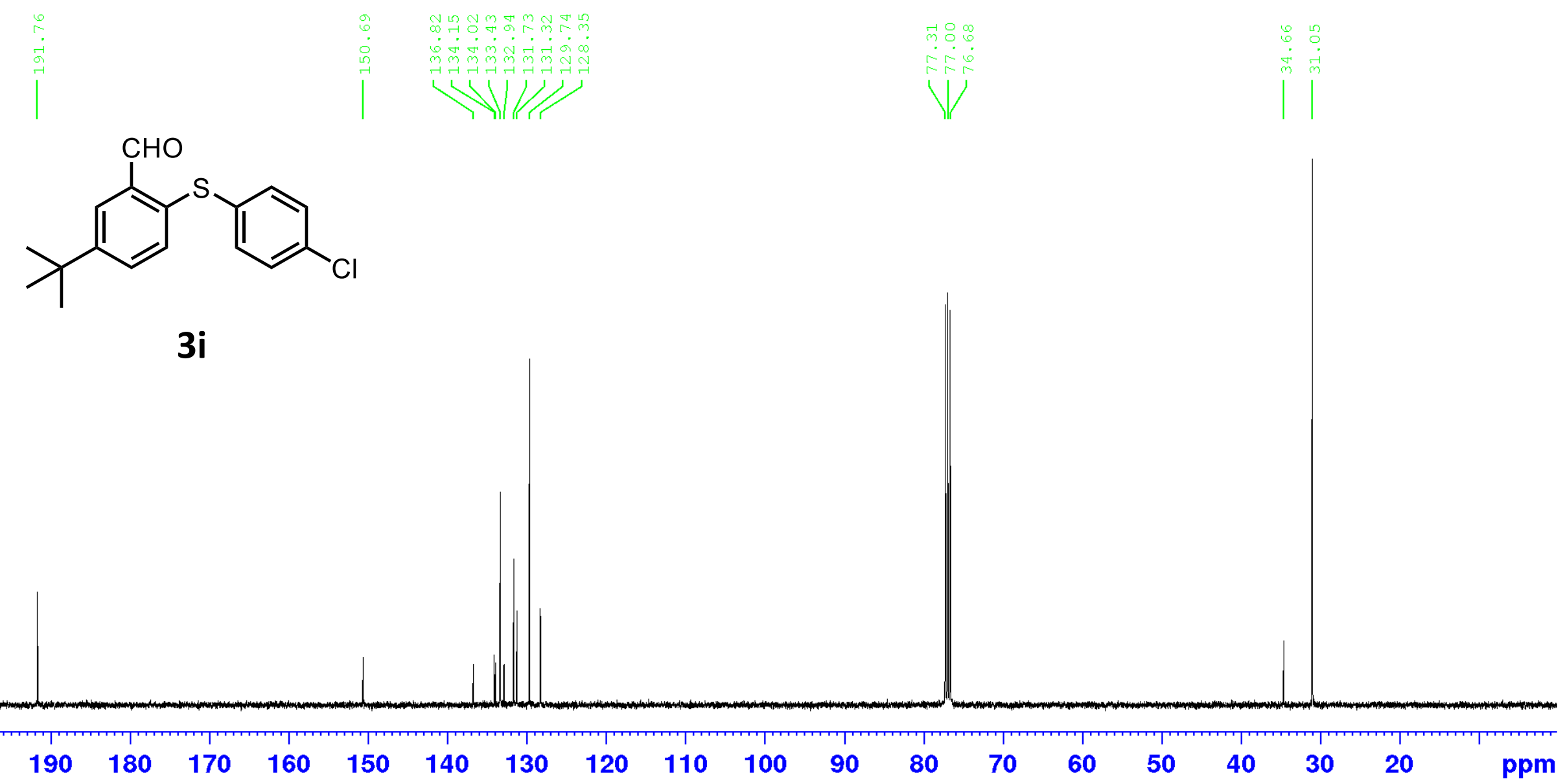


${ }^{1} \mathrm{H}$ NMR (400 MHz, $\left.\mathrm{CD}_{3} \mathrm{CN}, 298 \mathrm{~K}\right)$

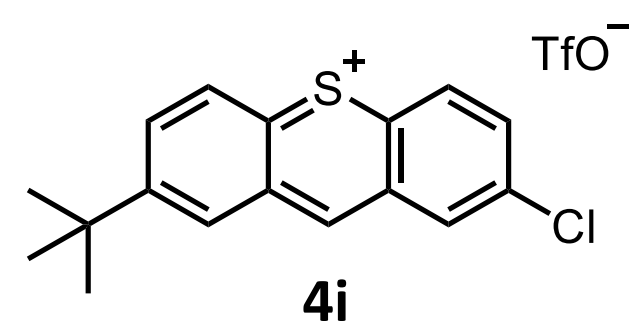

6
$\forall$ ㄱ.

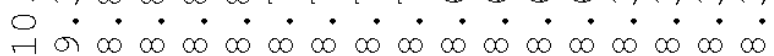
$\mathrm{L}_{\mathrm{L}}^{2} \mathrm{~L}$

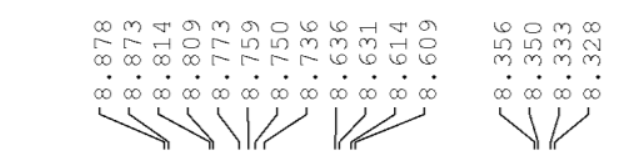

N $6 \circ+\infty$ म न न - $\cdot \cdot \cdot$. $\$$

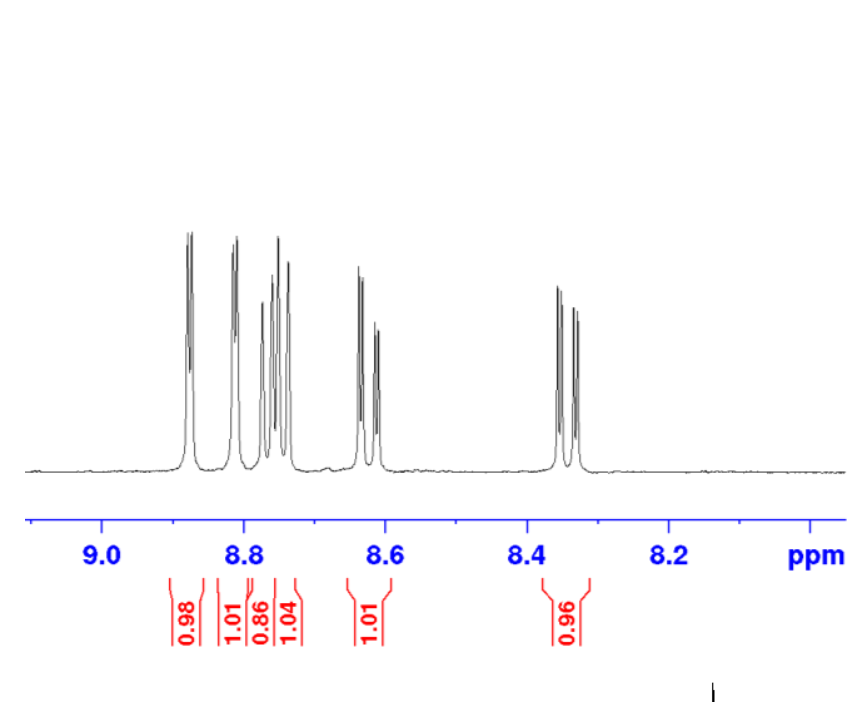

Hill "

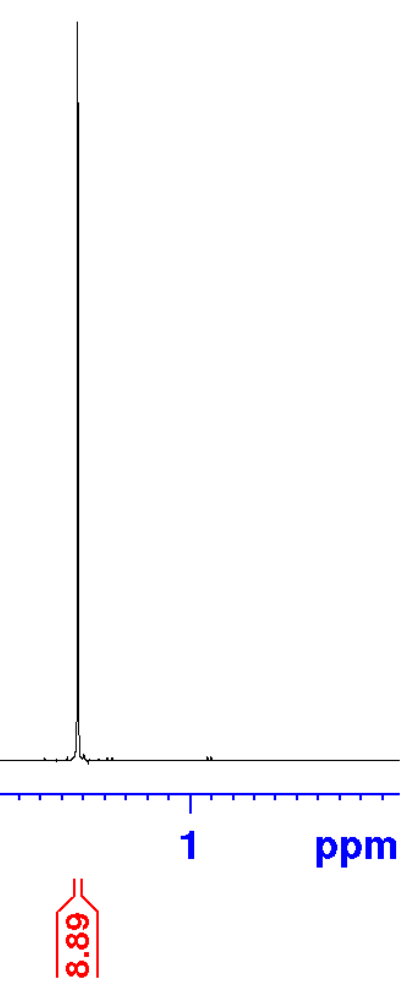


${ }^{13} \mathrm{C}\left\{{ }^{1} \mathrm{H}\right\} \mathrm{NMR}\left(101 \mathrm{MHz}, \mathrm{CD}_{3} \mathrm{CN}, 298 \mathrm{~K}\right)$<smiles>CC(C)(C)c1ccc2[s+]c3ccc(Cl)cc3cc2c1</smiles>

$4 i$

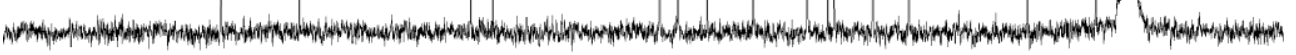

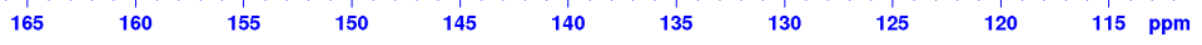


${ }^{1} \mathrm{H}$ NMR (400 MHz, $\mathrm{CDCl}_{3}, 298 \mathrm{~K}$ )
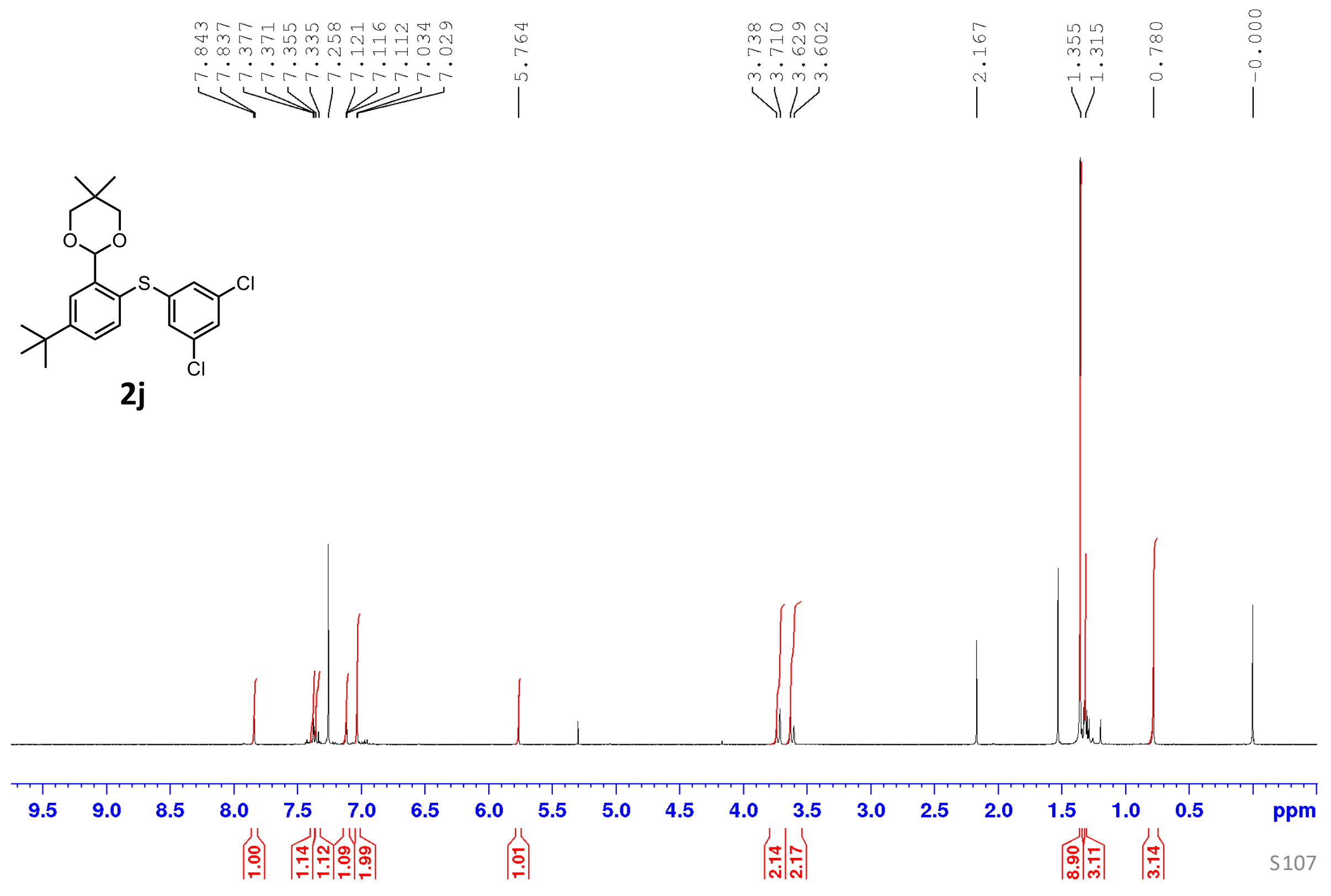
${ }^{13} \mathrm{C}\left\{{ }^{1} \mathrm{H}\right\}$ NMR (101 MHz, $\left.\mathrm{CDCl}_{3}, 298 \mathrm{~K}\right)$
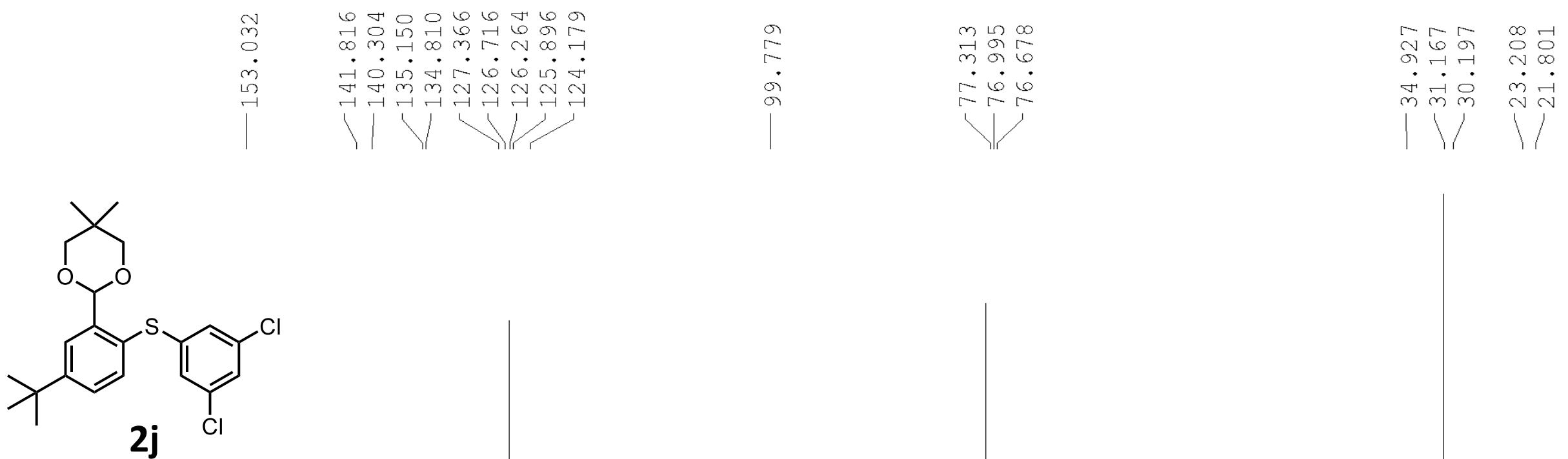

$2 \mathrm{j}$ 
${ }^{1} \mathrm{H}$ NMR (400 MHz, $\mathrm{CDCl}_{3}, 298 \mathrm{~K}$ )
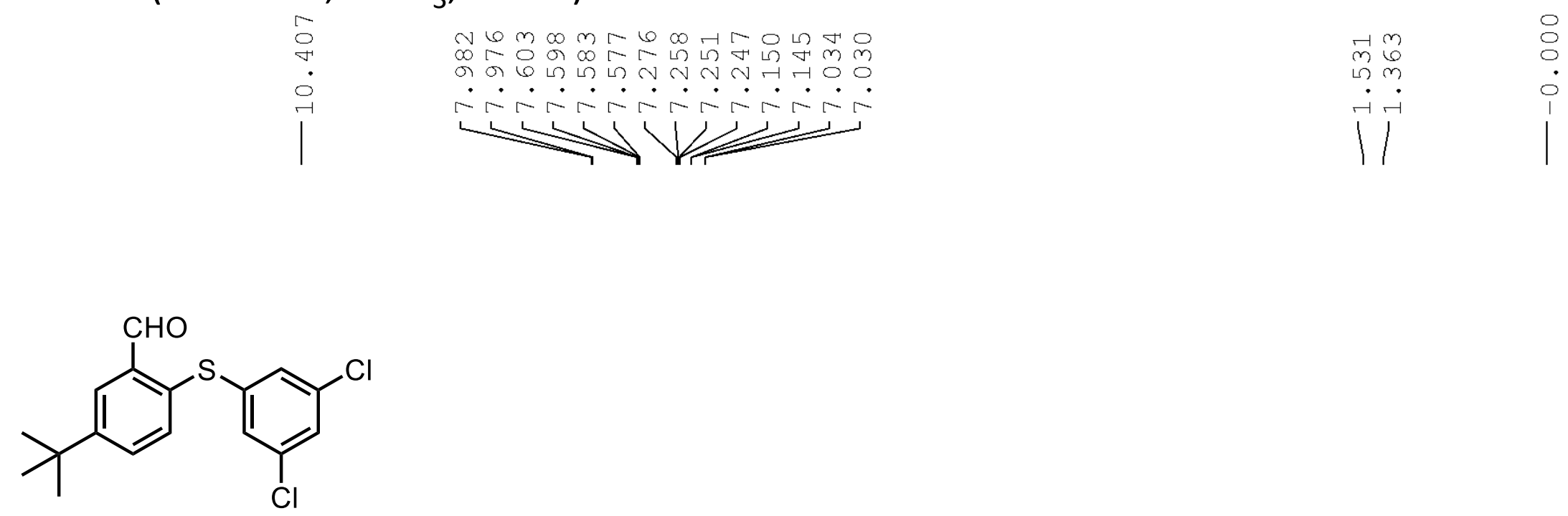

3j

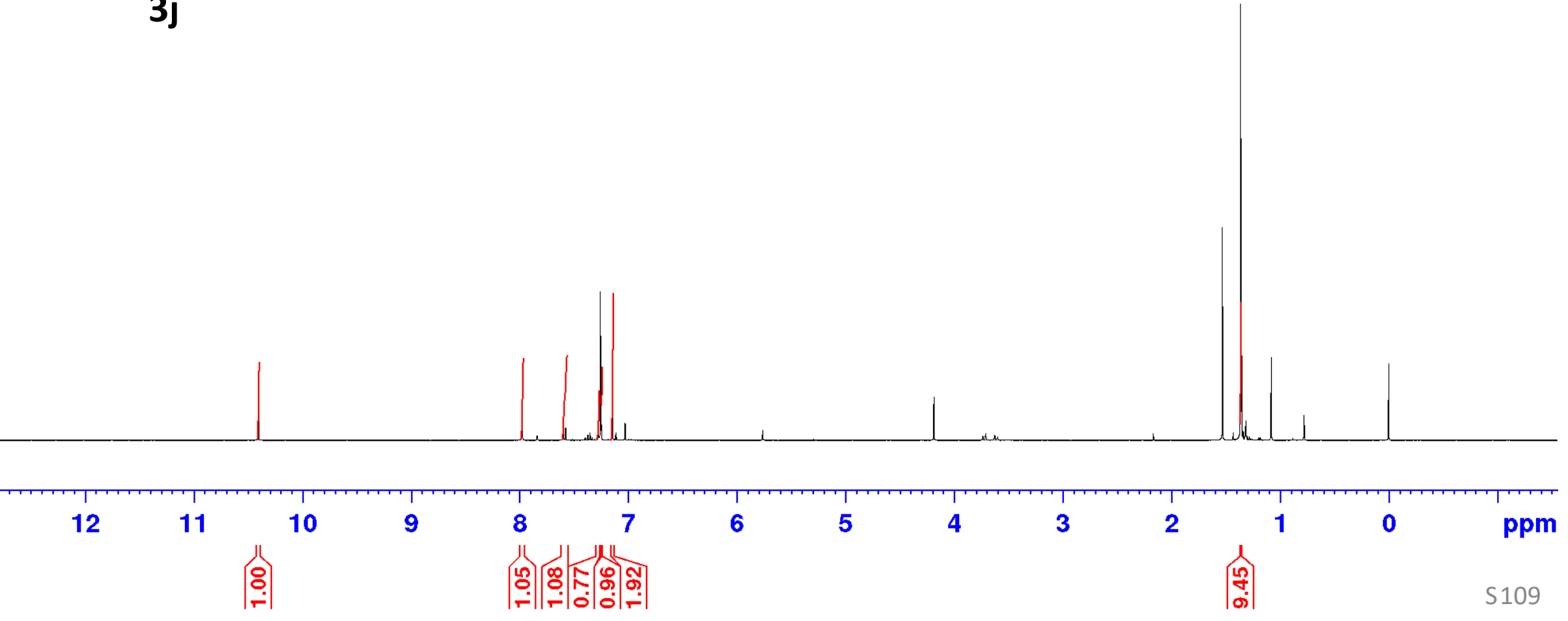


${ }^{13} \mathrm{C}\left\{{ }^{1} \mathrm{H}\right\} \mathrm{NMR}\left(101 \mathrm{MHz}, \mathrm{CDCl}_{3}, 298 \mathrm{~K}\right)$
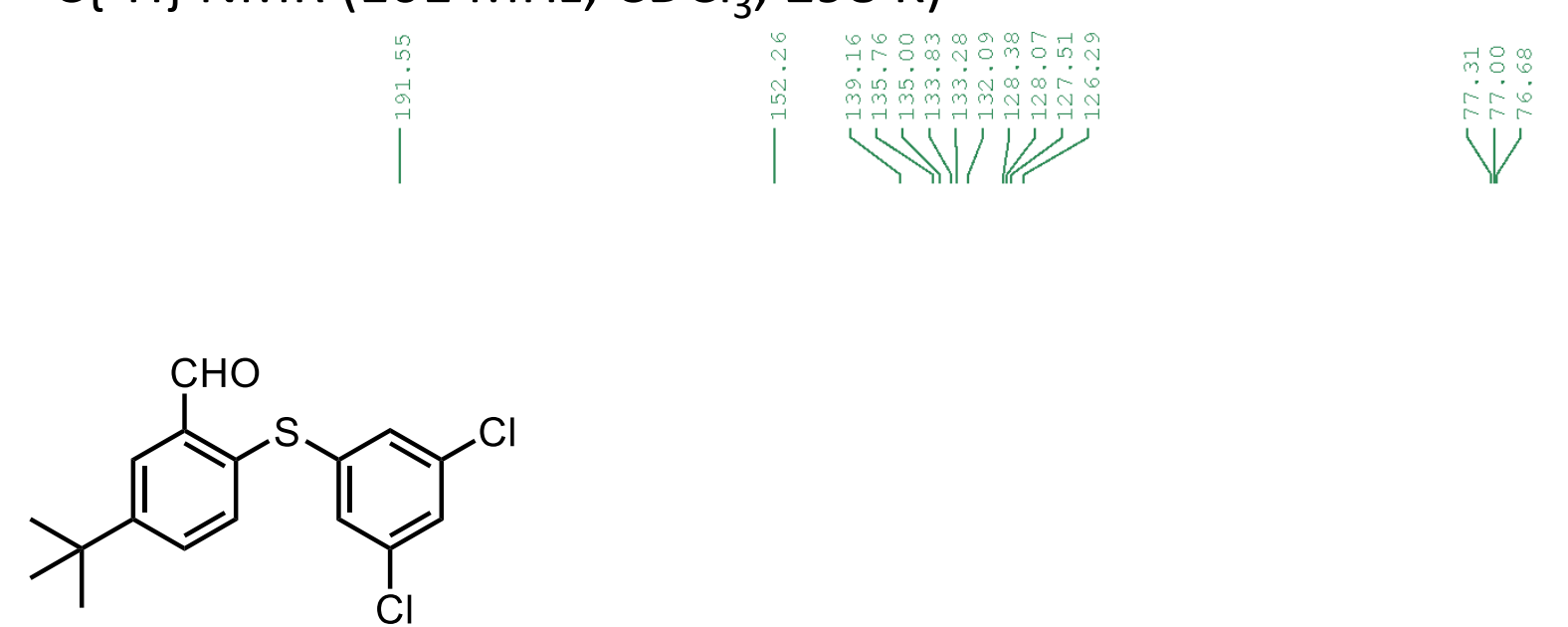

3j

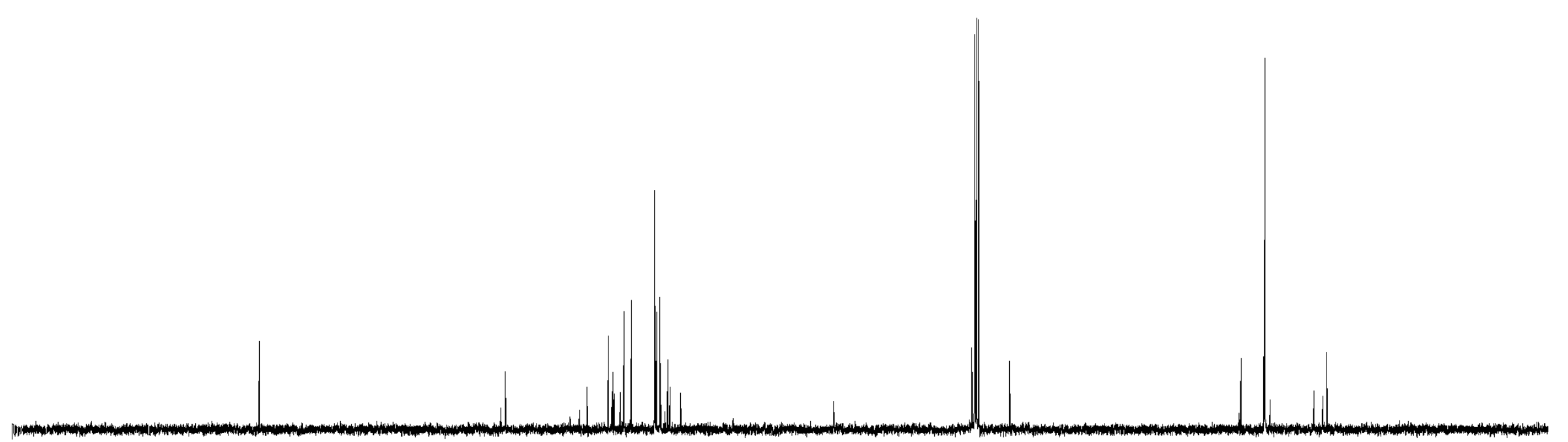

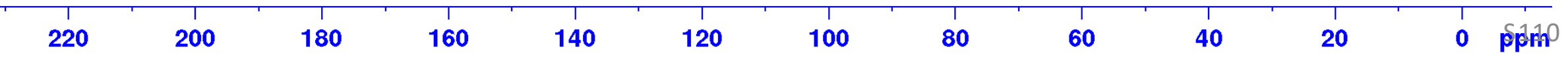


${ }^{1} \mathrm{H}$ NMR (400 MHz, $\left.\mathrm{CD}_{3} \mathrm{CN}, 298 \mathrm{~K}\right)$
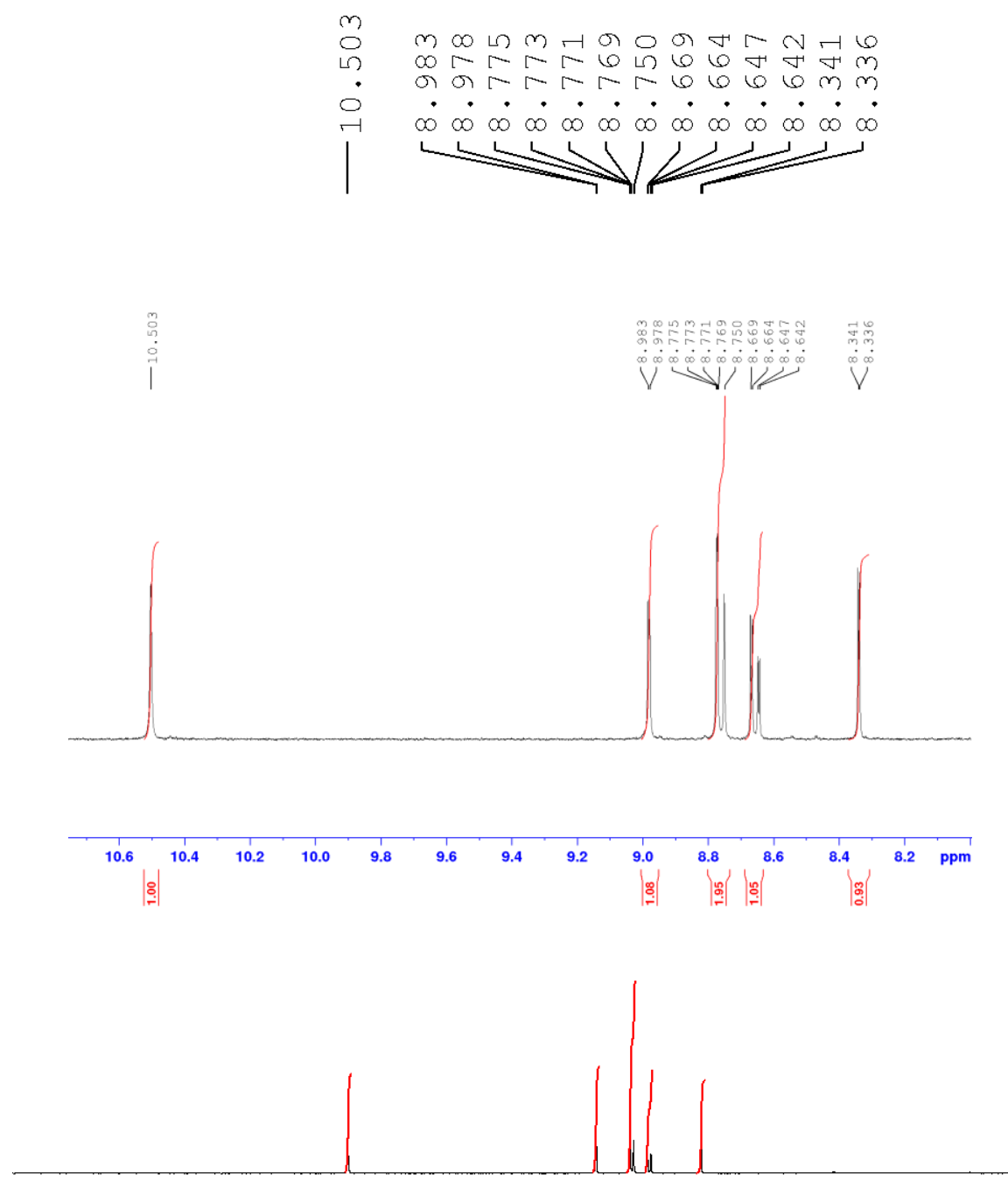

12
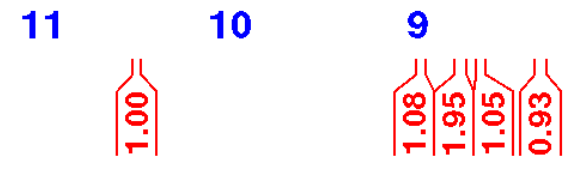

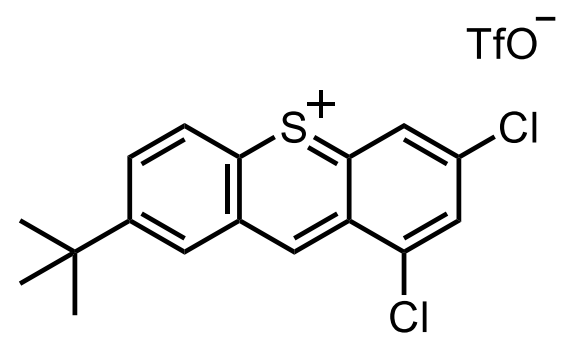

$4 \mathbf{j}$

Cl
$N \in \circ+4 \infty$

न न न न เ เ

桨

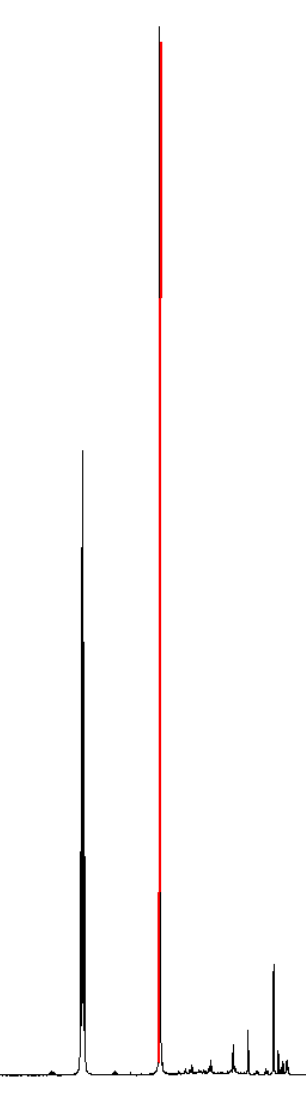

6

5

4

3

2

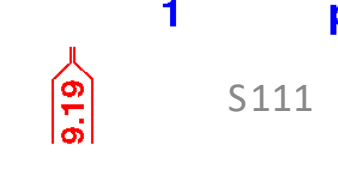


${ }^{13} \mathrm{C}\left\{{ }^{1} \mathrm{H}\right\}$ NMR $\left(101 \mathrm{MHz}, \mathrm{CD}_{3} \mathrm{CN}, 298 \mathrm{~K}\right)$

VIIIVViVil

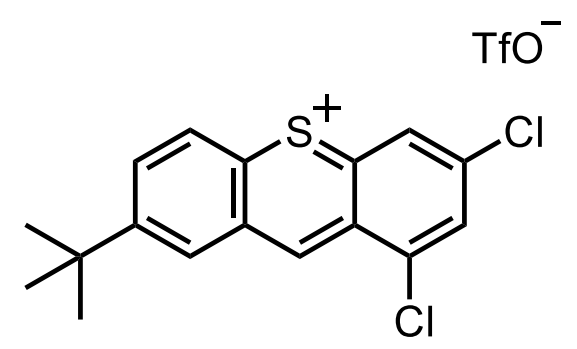

4j
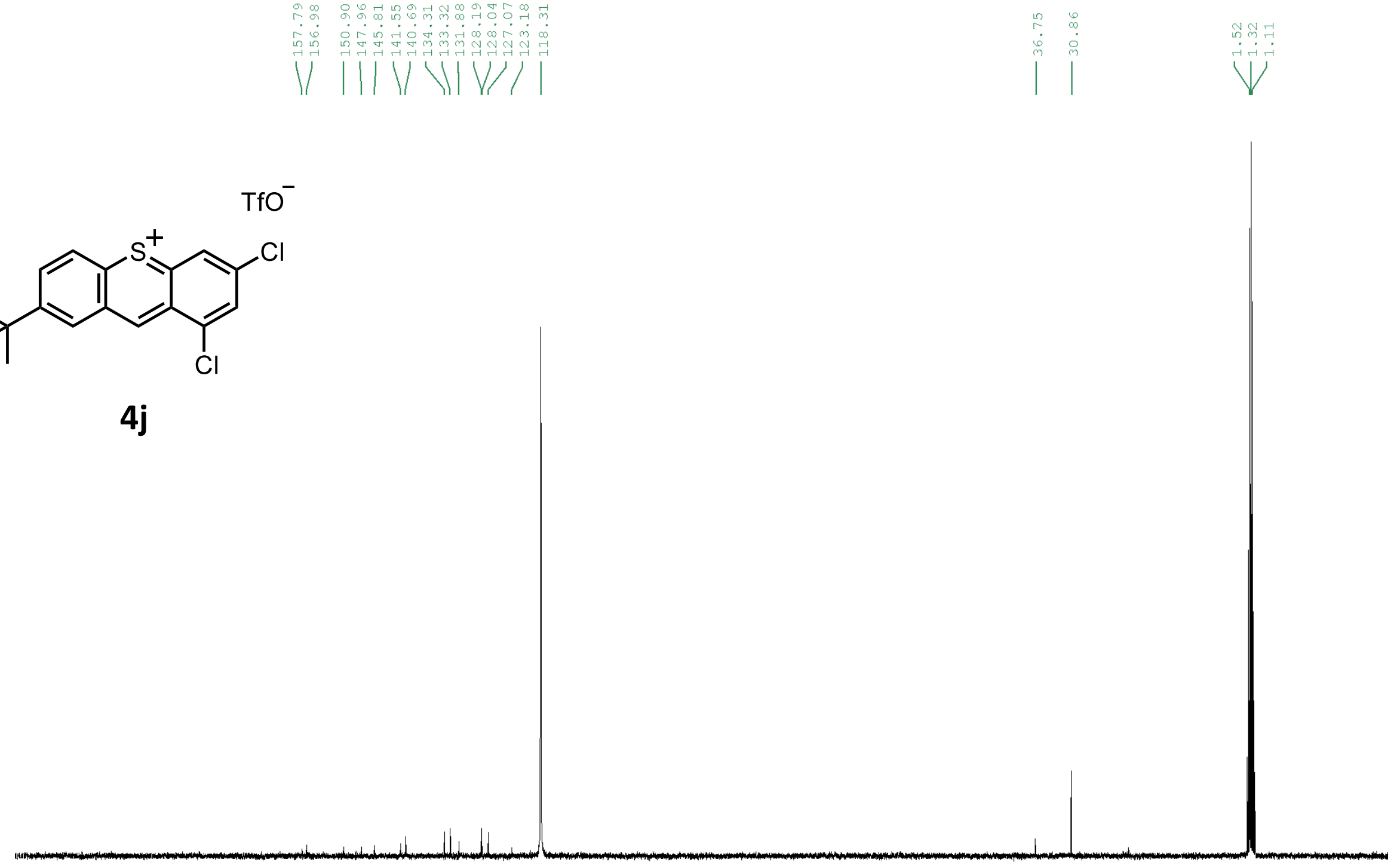

$\begin{array}{llllllllllllllllllllll}200 & 190 & 180 & 170 & 160 & 150 & 140 & 130 & 120 & 110 & 100 & 90 & 80 & 70 & 60 & 50 & 40 & 30 & 20 & 10 & 0 & \text { SPPm }\end{array}$ 
${ }^{1} \mathrm{H} \mathrm{NMR}\left(400 \mathrm{MHz}, \mathrm{CDCl}_{3}, 298 \mathrm{~K}\right.$ )

ㄴ

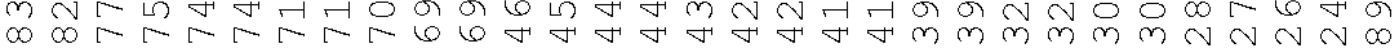

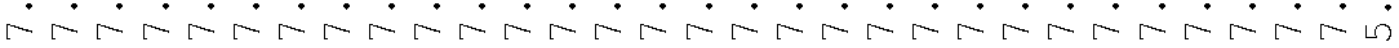

न

대

$\dot{m} \dot{m} \dot{m}$

$1 /$
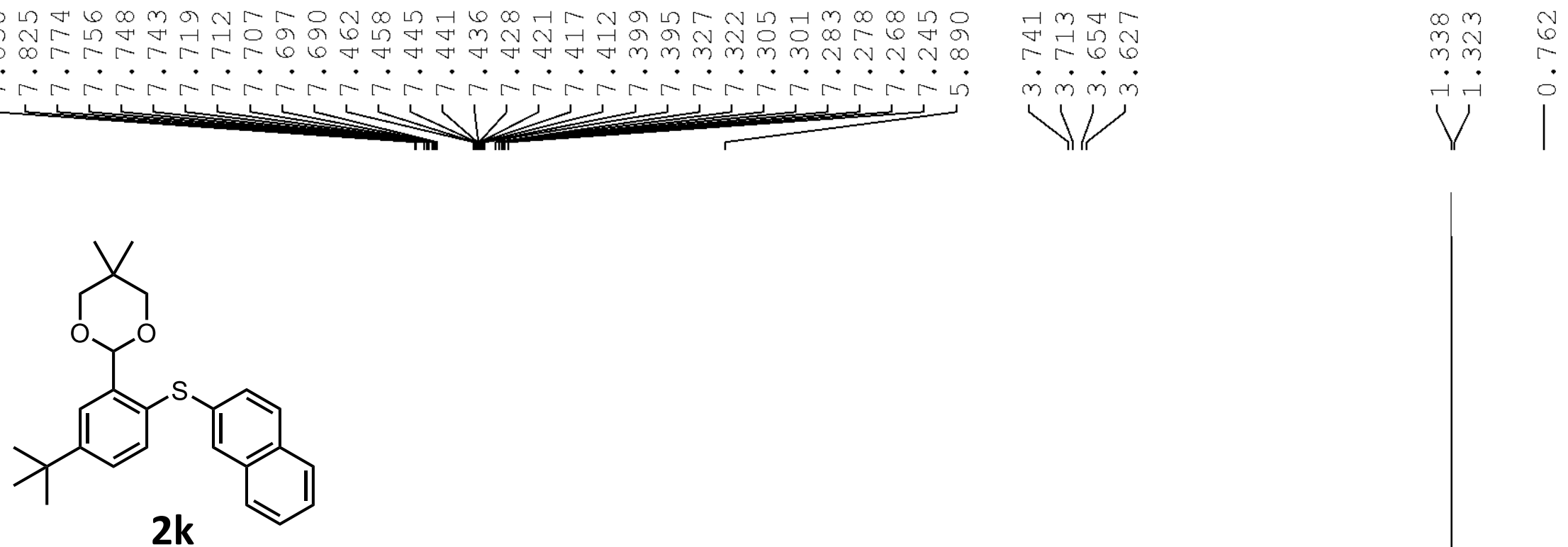

TIII

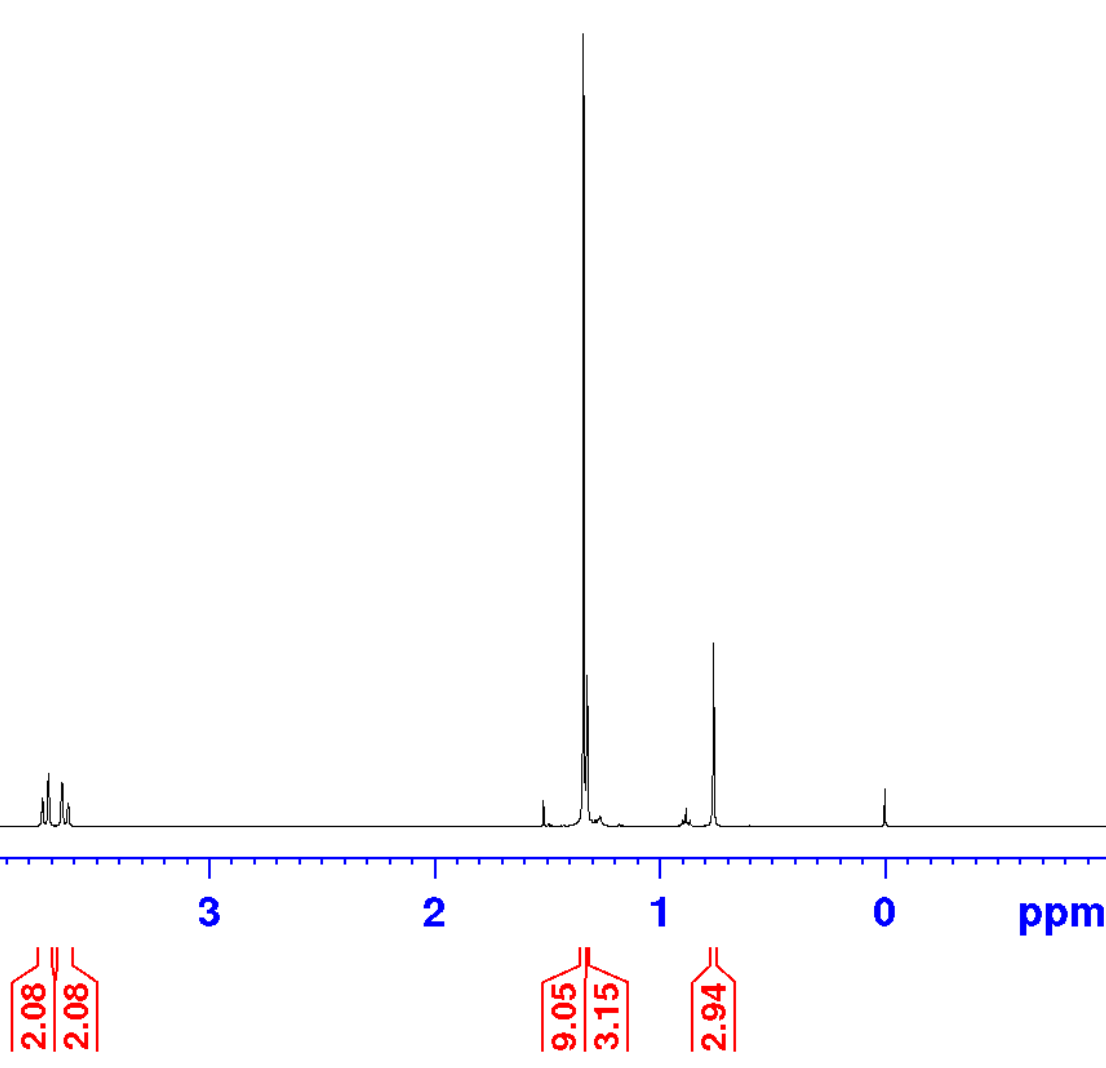


${ }^{13} \mathrm{C}\left\{{ }^{1} \mathrm{H}\right\}$ NMR (101 MHz, $\left.\mathrm{CDCl}_{3}, 298 \mathrm{~K}\right)$
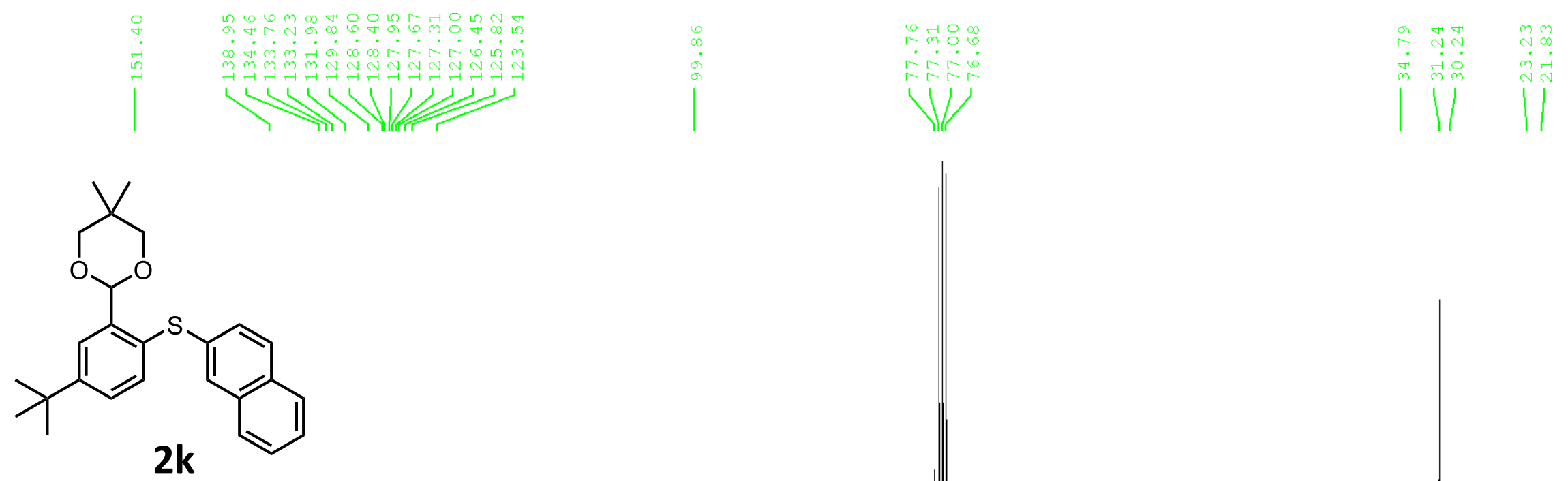
${ }^{1} \mathrm{H}$ NMR (400 MHz, $\mathrm{CDCl}_{3}, 298 \mathrm{~K}$ )

Ğ

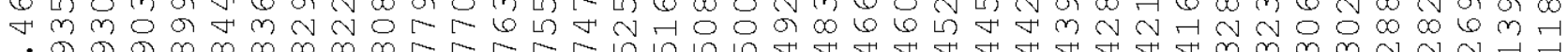

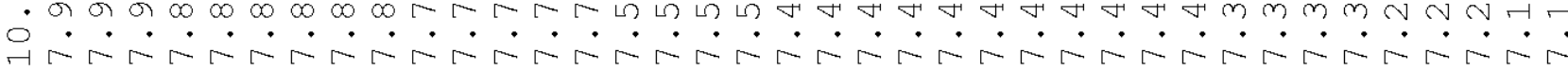

L

inim

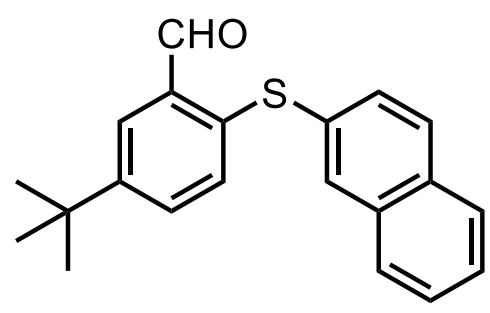

3k

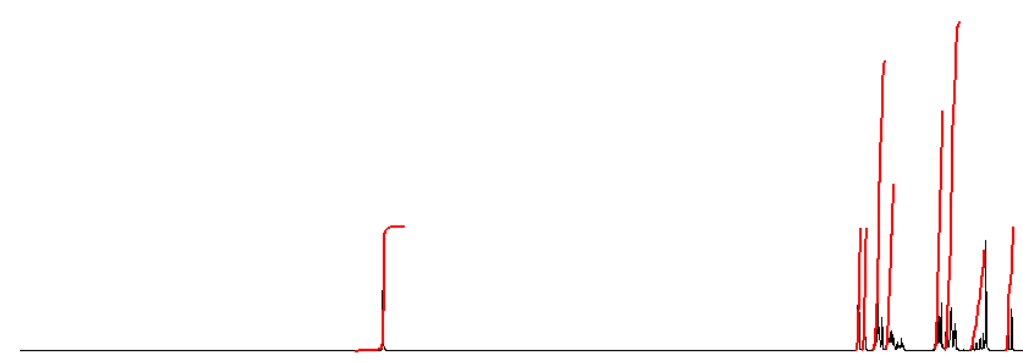

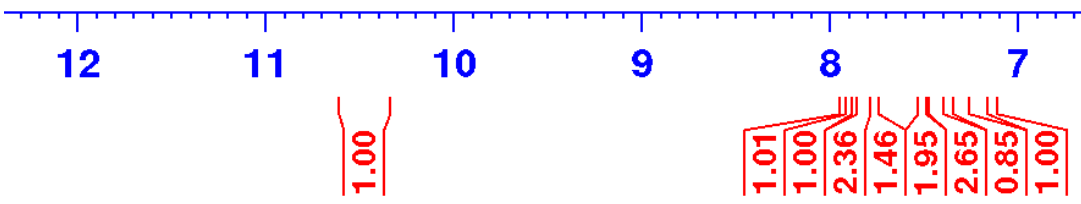

6

5

4

3

2

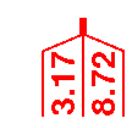

o ppm 
${ }^{13} \mathrm{C}\left\{{ }^{1} \mathrm{H}\right\} \mathrm{NMR}\left(101 \mathrm{MHz}, \mathrm{CDCl}_{3}, 298 \mathrm{~K}\right)$
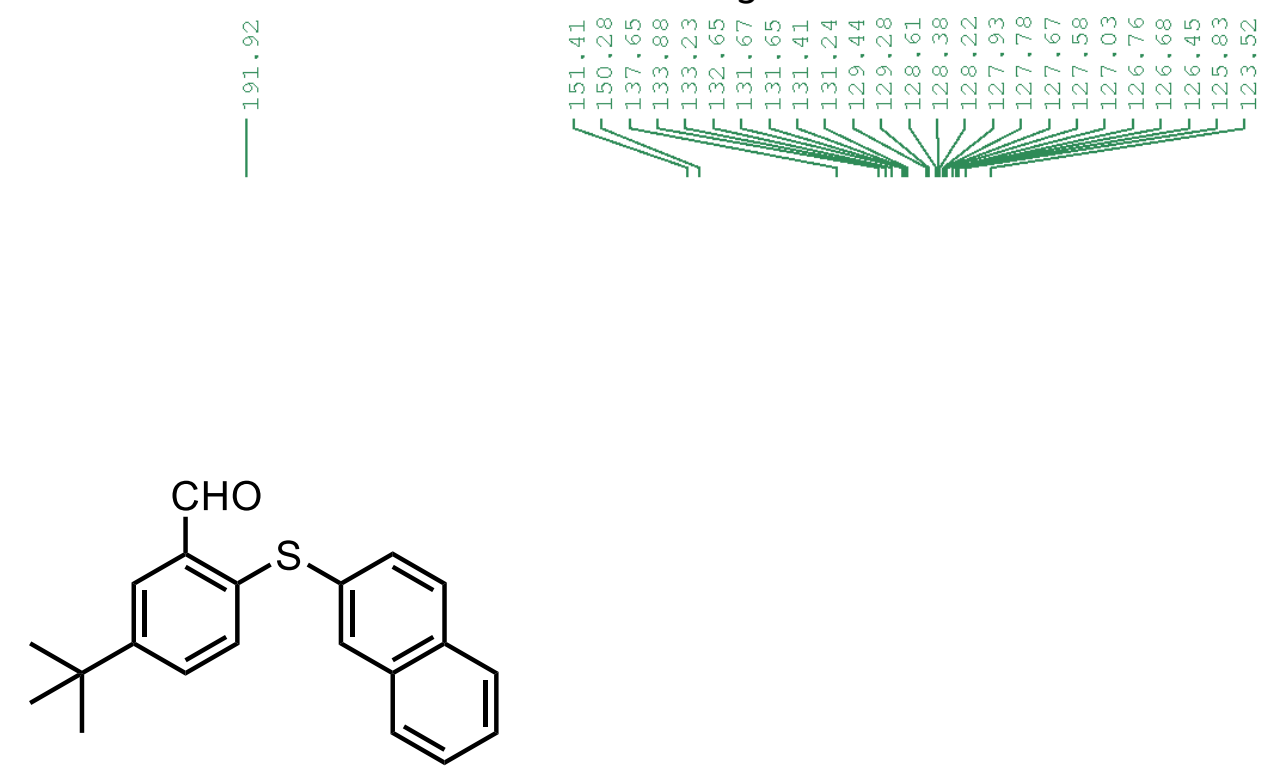

$3 k$

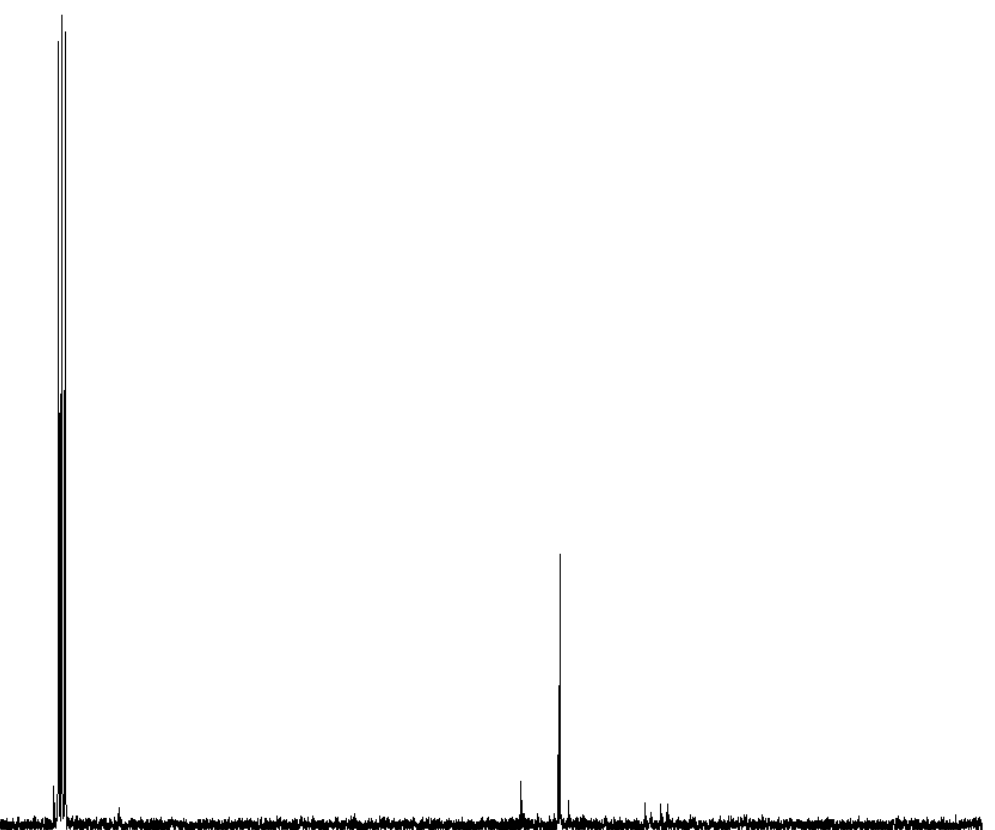


${ }^{1} \mathrm{H}$ NMR (400 MHz, CD 3 CN, $\left.298 \mathrm{~K}\right)$

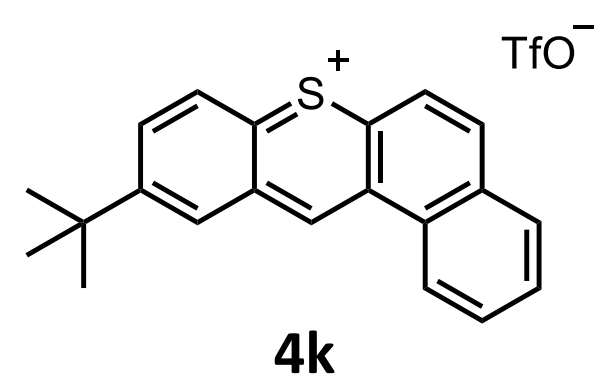

inक न

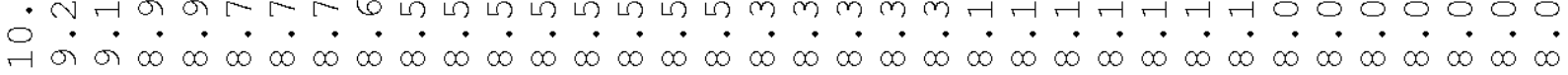

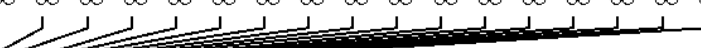

N のののの レ $\dot{i} \dot{-} \dot{-} \dot{-} \dot{i}$ $+2$

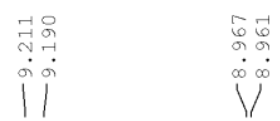

$\int_{1}^{\infty}$
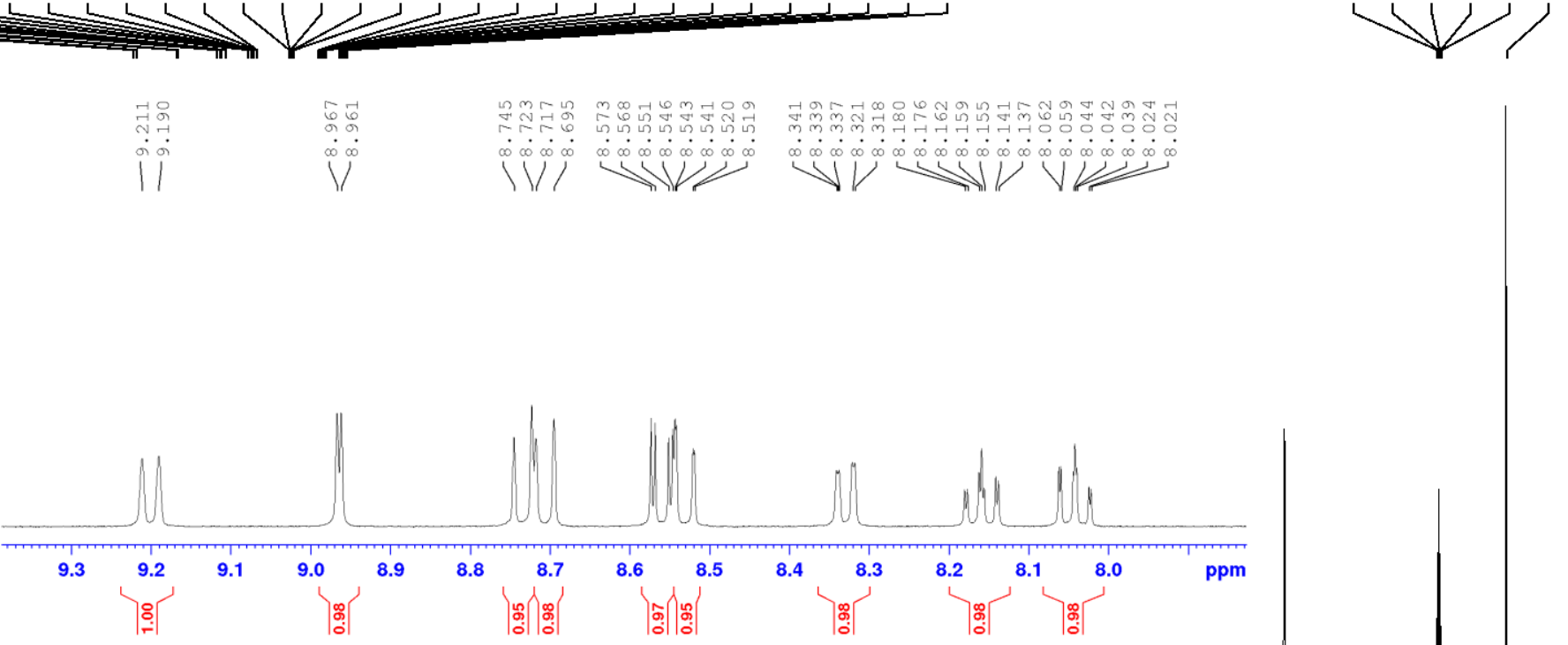

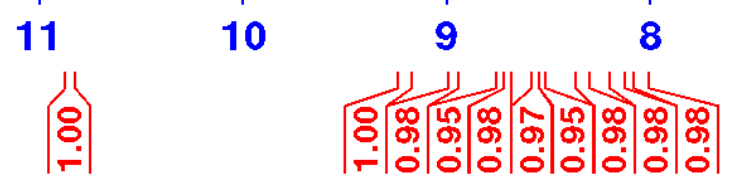

7

6

5

4

3 尔 
${ }^{13} \mathrm{C}\left\{{ }^{1} \mathrm{H}\right\}$ NMR (101 MHz, CD $\left.\mathrm{CN}, 298 \mathrm{~K}\right)$
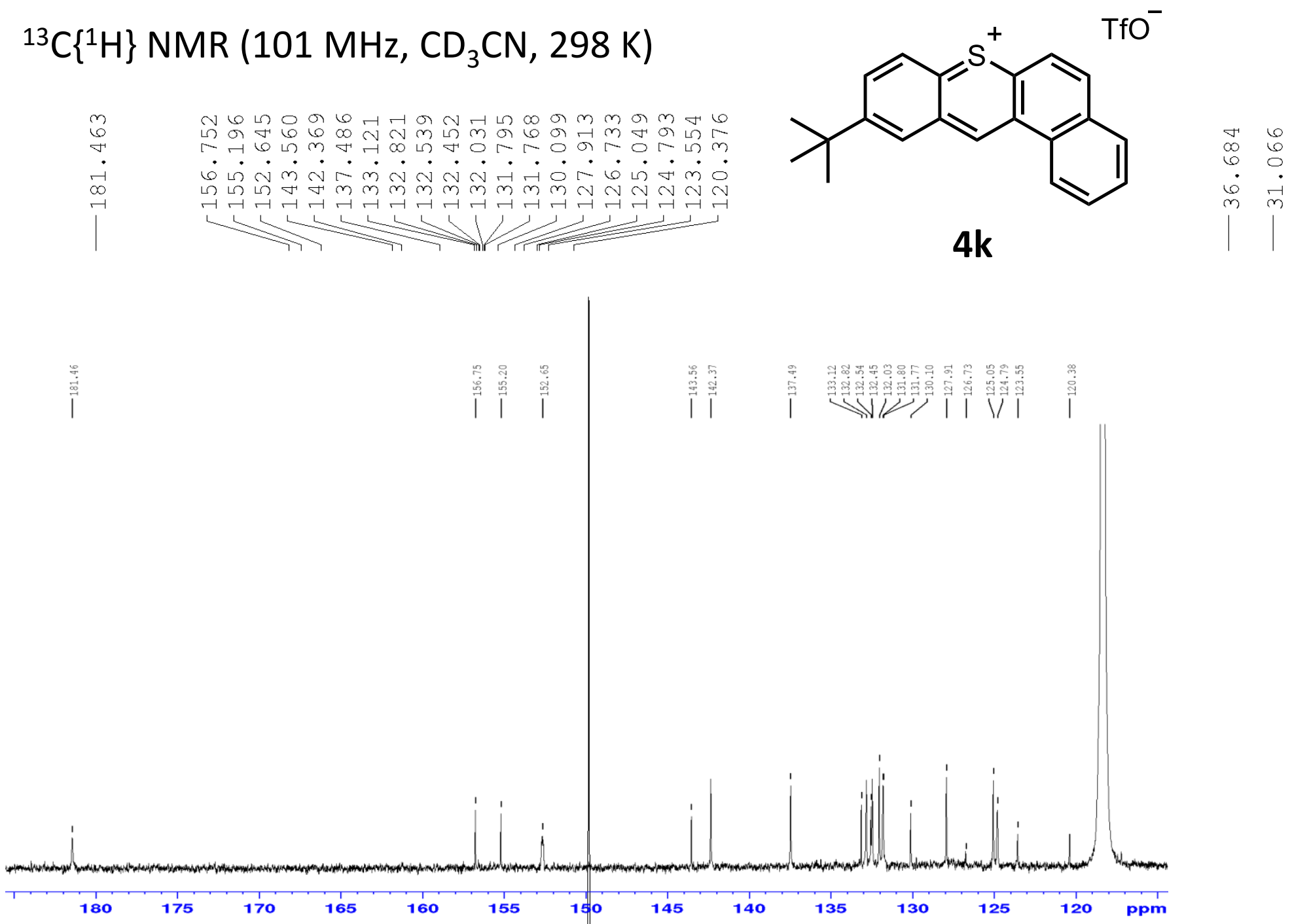
${ }^{13} \mathrm{C}\left\{{ }^{1} \mathrm{H}\right\}$ NMR $\left(101 \mathrm{MHz}, \mathrm{CDCl}_{3}, 298 \mathrm{~K}\right)$
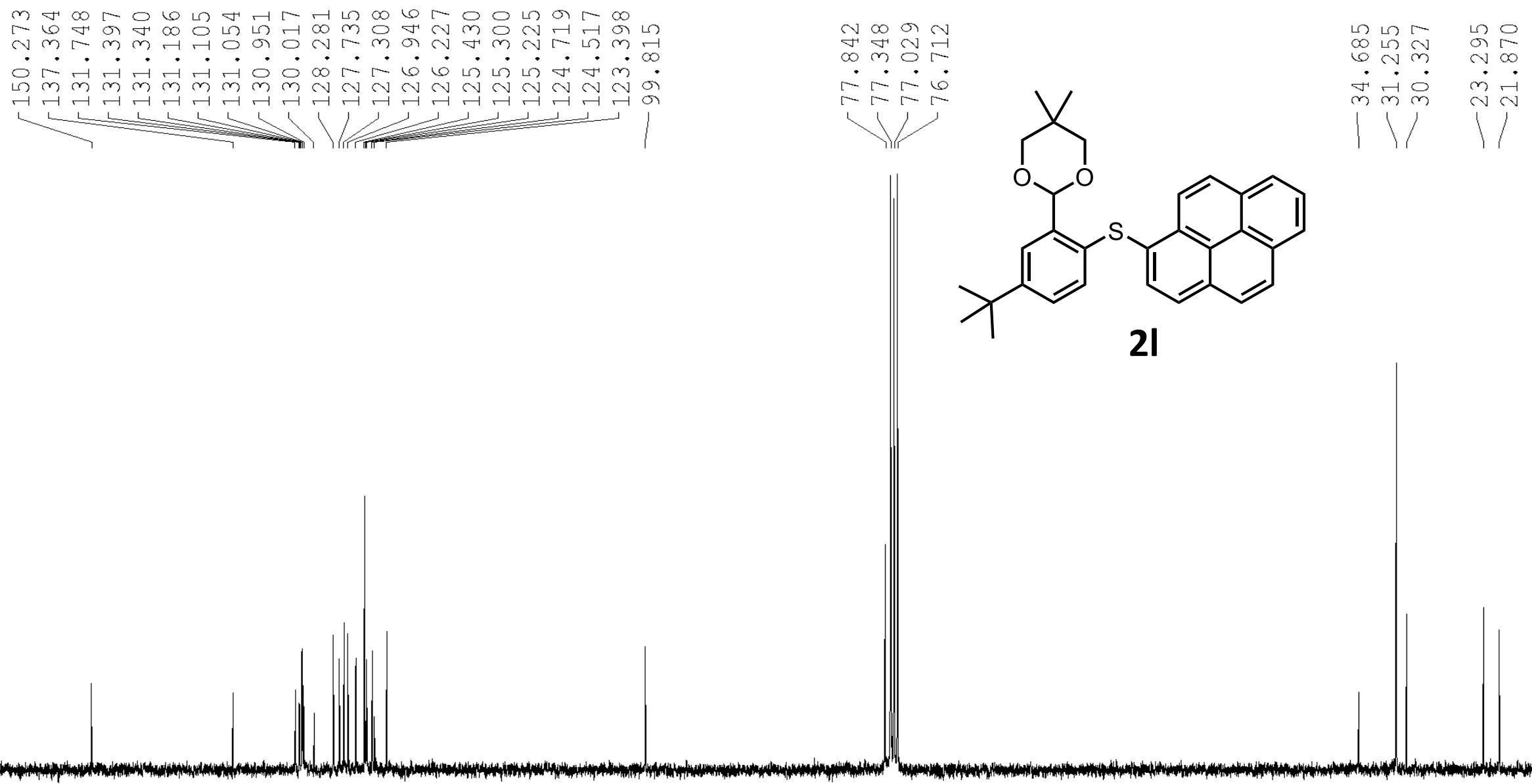

140

130

120

110

100

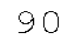

80

70

60

50

40

30

10 
${ }^{1} \mathrm{H}$ NMR (400 MHz, $\left.\mathrm{CD}_{3} \mathrm{CN}, 298 \mathrm{~K}\right)$

$\mathrm{TfO}^{-}$

감웜ㄷㄲ

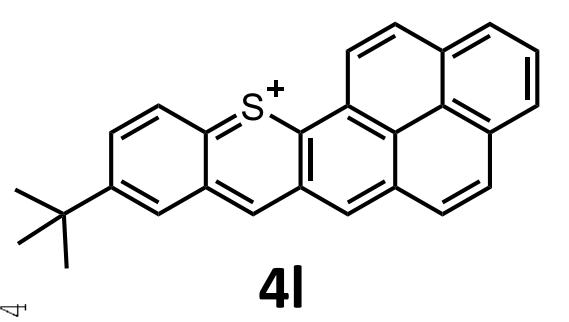

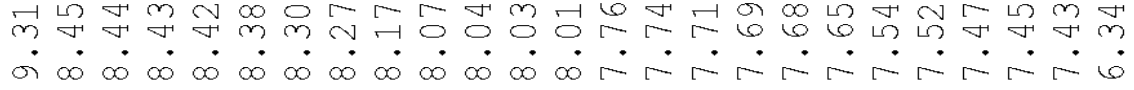

(1)
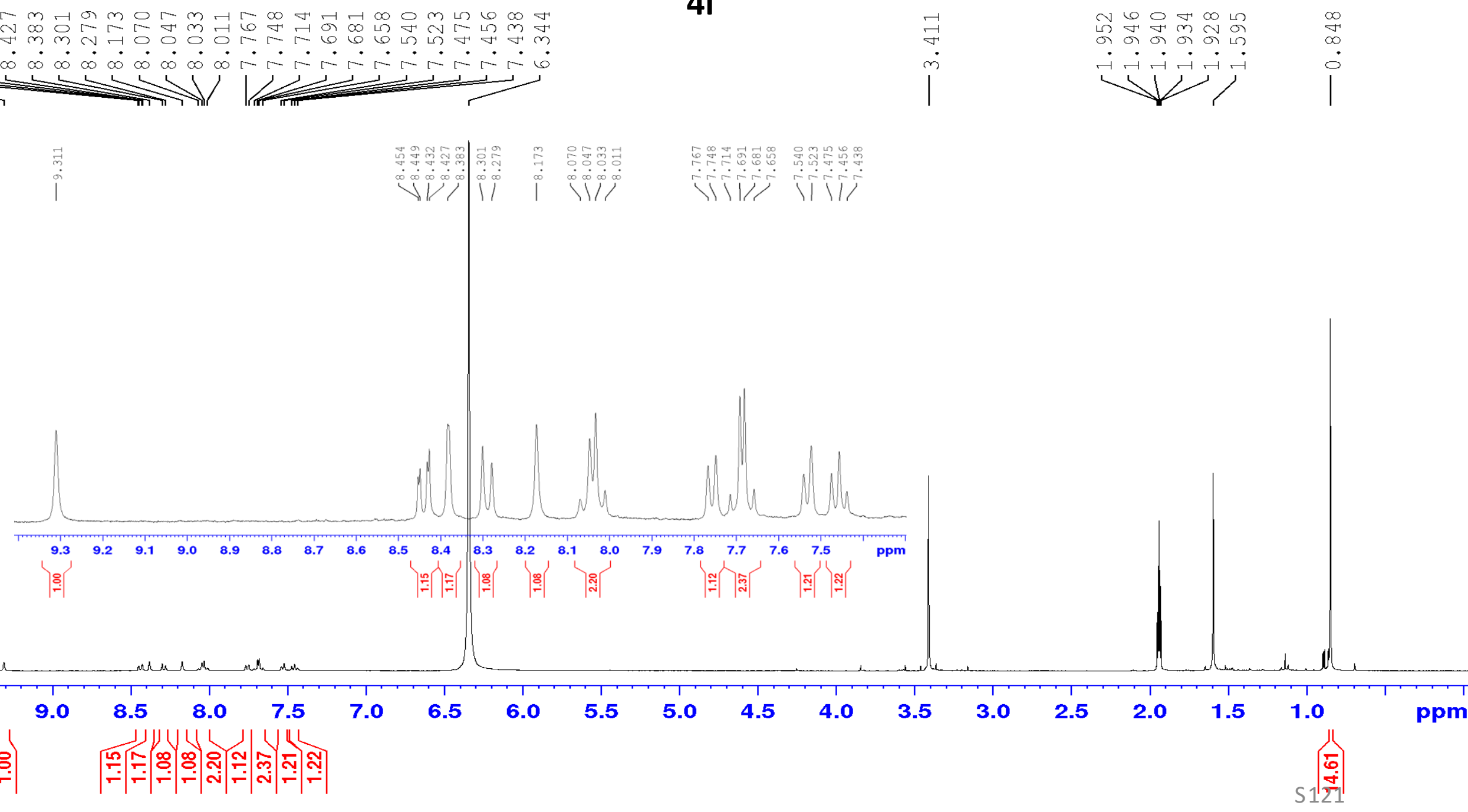
${ }^{1} \mathrm{H}$ NMR (400 MHz, $\left.\mathrm{CDCl}_{3}, 298 \mathrm{~K}\right)$

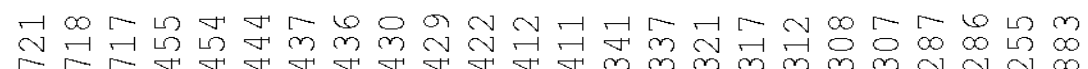

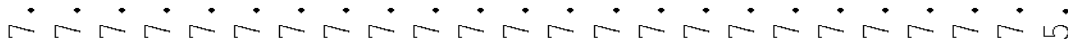

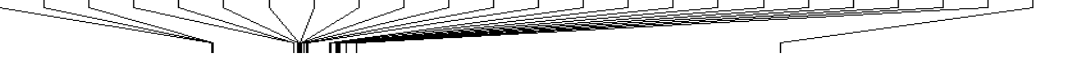

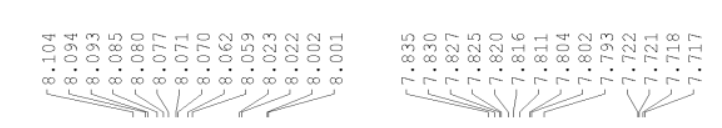
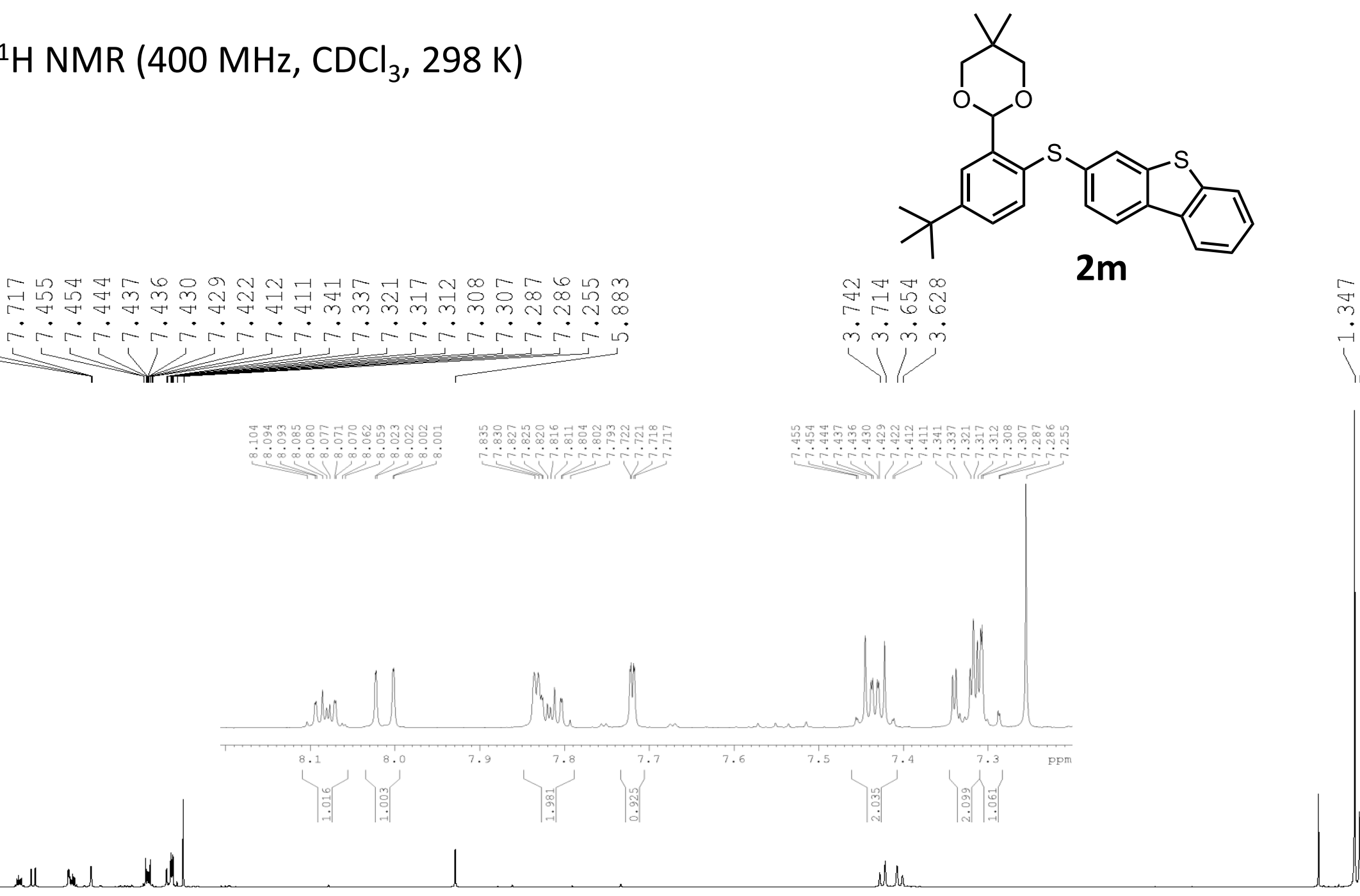

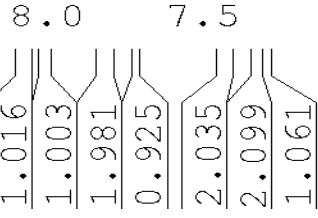

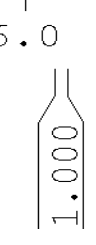

5.5

5.0

$4 \cdot 5$

4.0
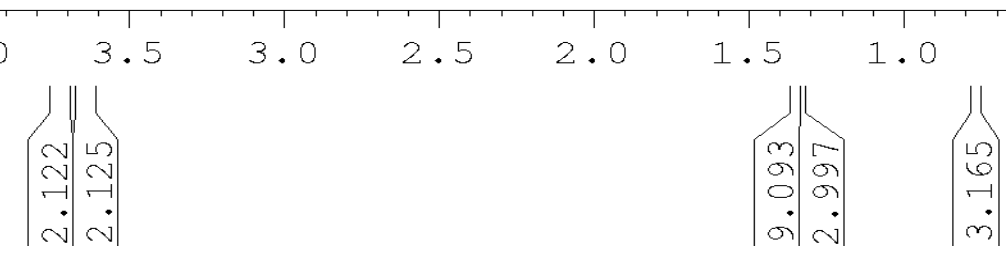
${ }^{13} \mathrm{C}\left\{{ }^{1} \mathrm{H}\right\}$ NMR (101 MHz, $\left.\mathrm{CDCl}_{3}, 298 \mathrm{~K}\right)$

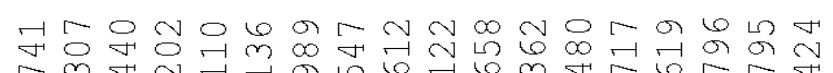

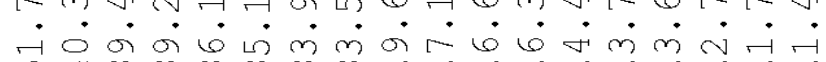

막 m m m m m

$\longrightarrow 1$

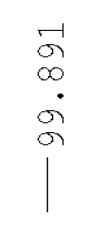

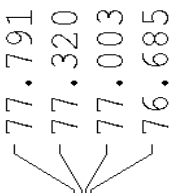

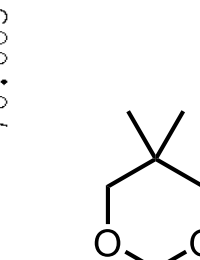

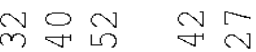

$\infty \sim \sim \sim \infty$

मेंमें $\dot{m} \dot{\sim}$

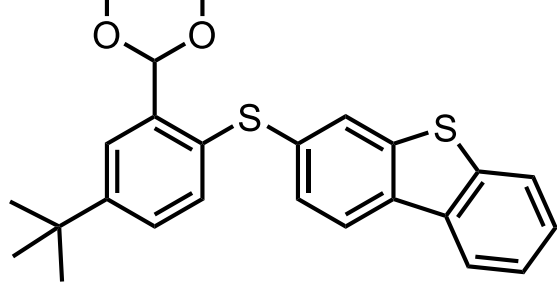

$2 m$ 
${ }^{1} \mathrm{H}$ NMR (400 MHz, CD $\mathrm{CN}, 298 \mathrm{~K}$ )
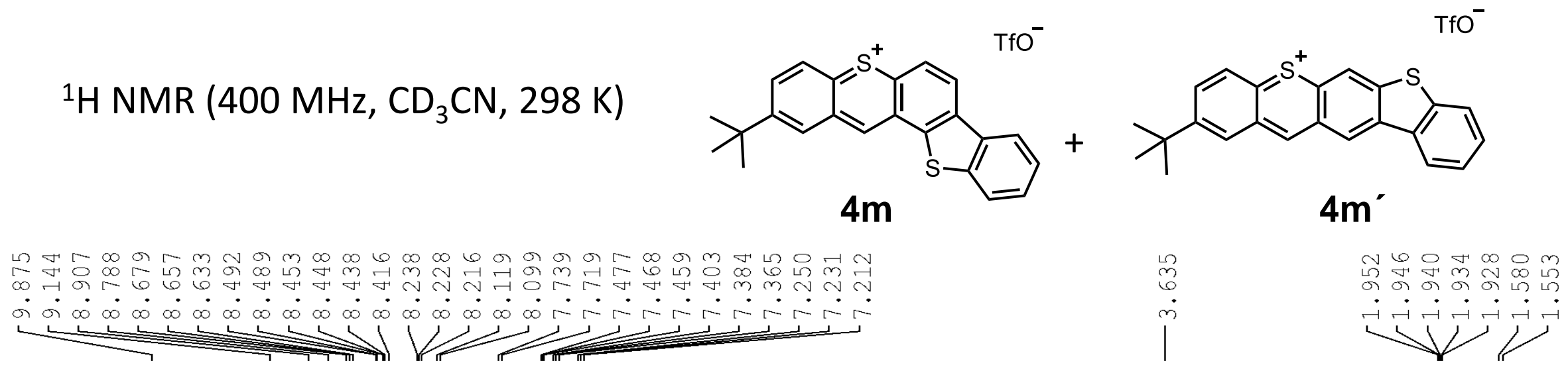

p

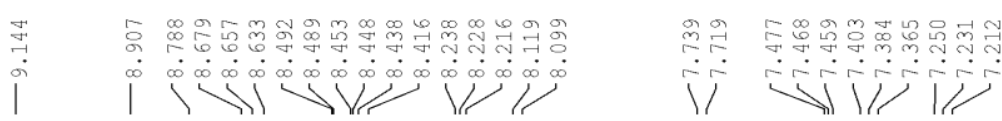

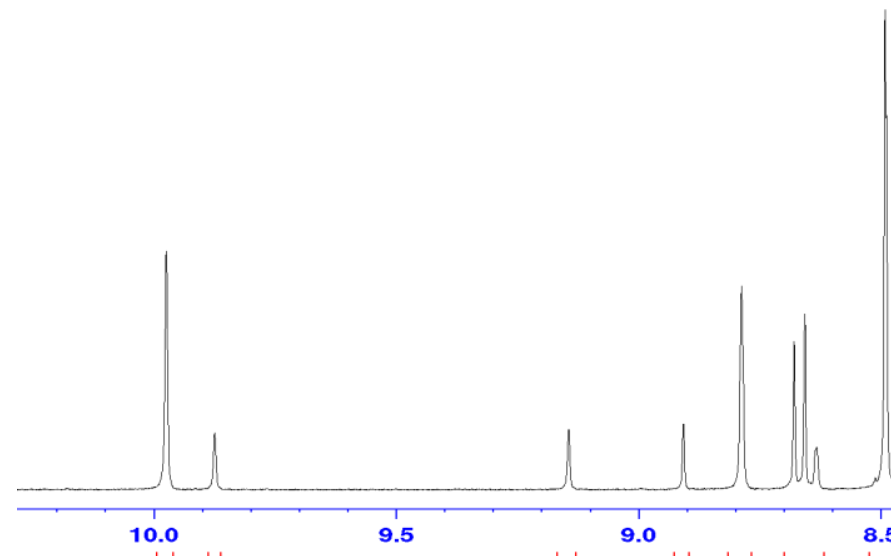

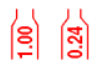

|พั่

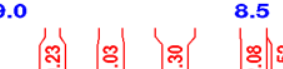

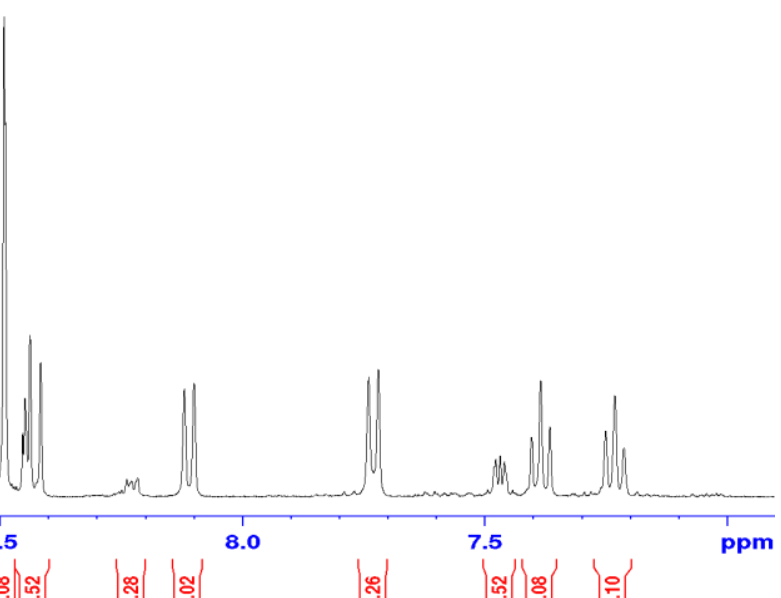

1

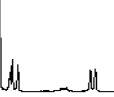

8

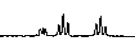

76

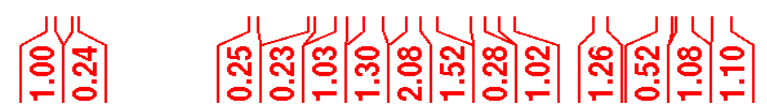


${ }^{13} \mathrm{C}\left\{{ }^{1} \mathrm{H}\right\}$ NMR (101 MHz, CD 3 CN, $\left.298 \mathrm{~K}\right)$

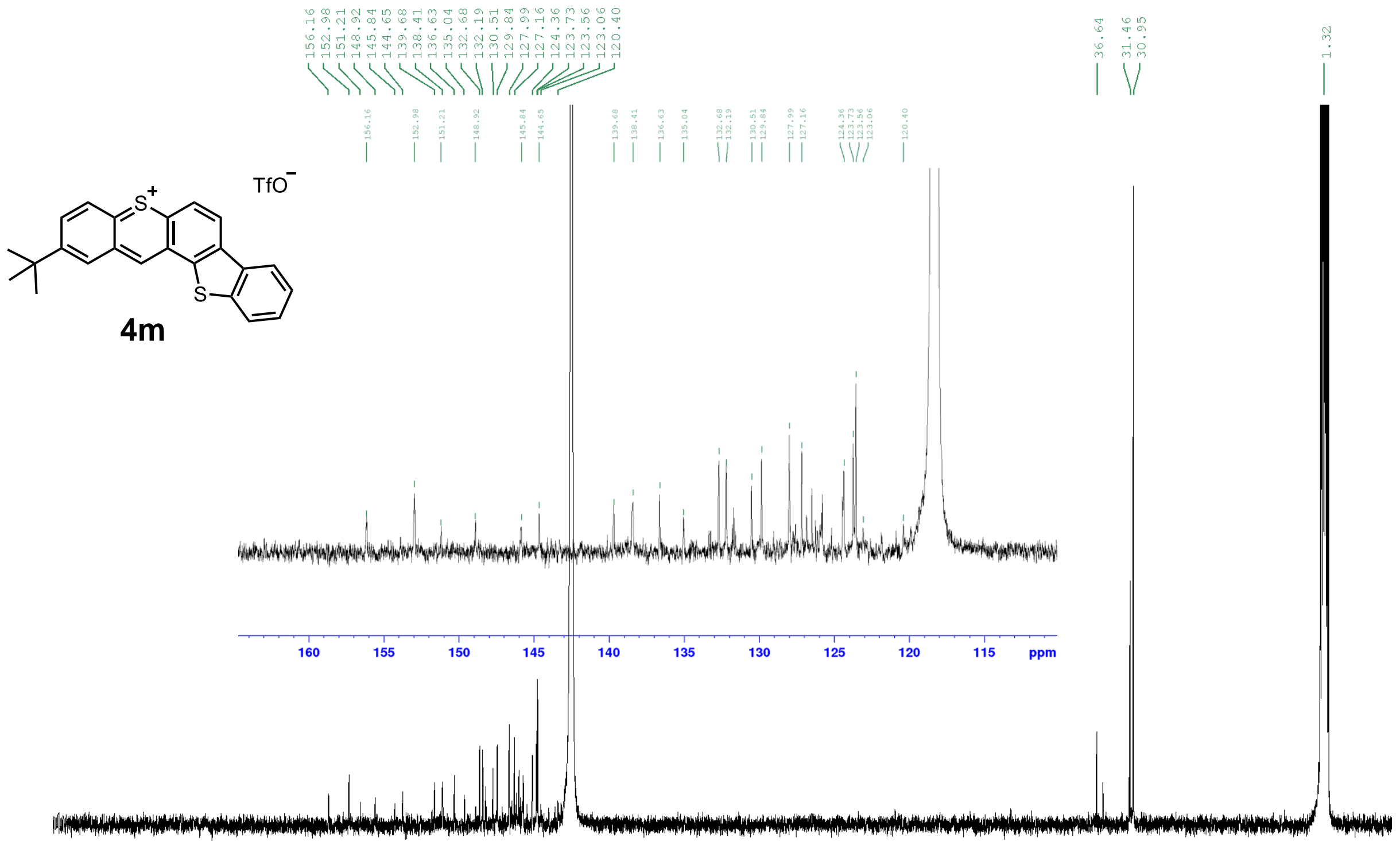


${ }^{13} \mathrm{C}\left\{{ }^{1} \mathrm{H}\right\}$ NMR (101 MHz, $\left.\mathrm{CDCl}_{3}, 298 \mathrm{~K}\right)$

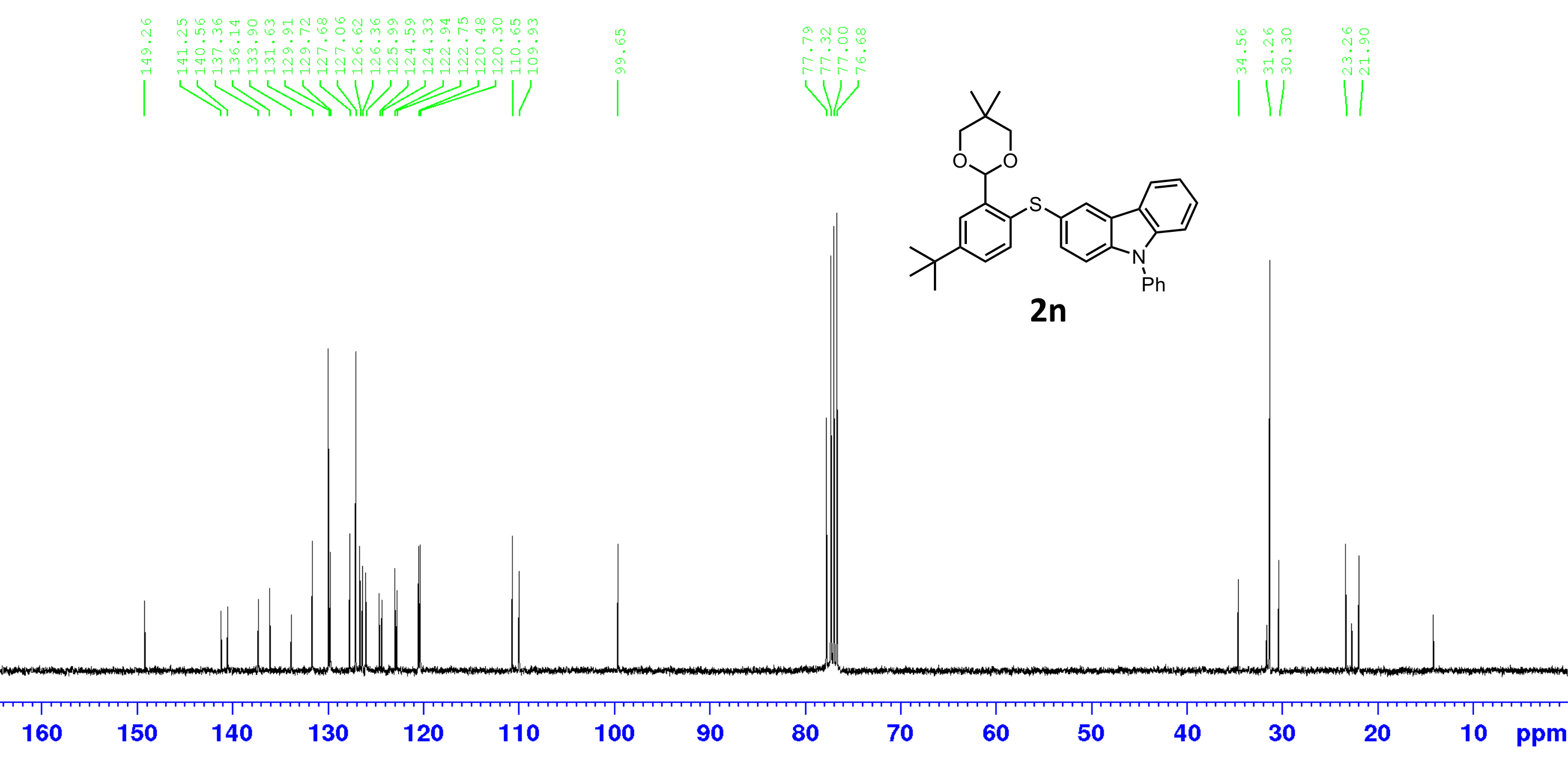


${ }^{1} \mathrm{H}$ NMR (400 MHz, $\left.\mathrm{CD}_{3} \mathrm{CN}, 298 \mathrm{~K}\right)$

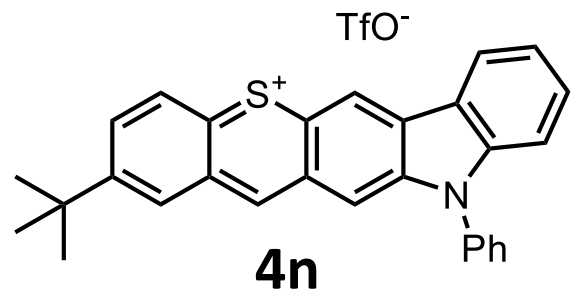

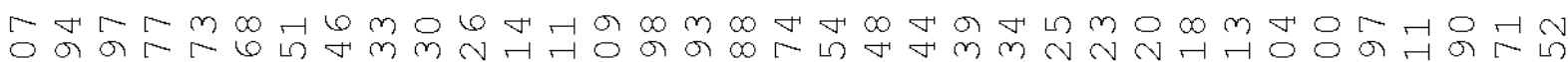

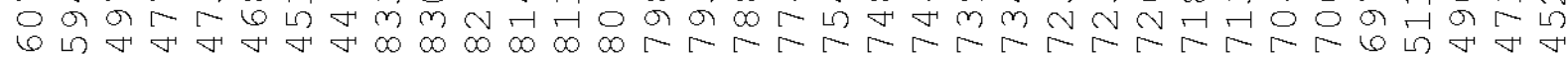

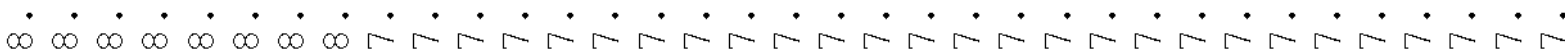

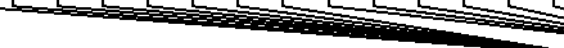

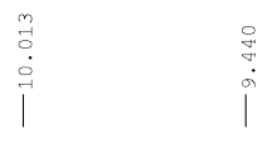

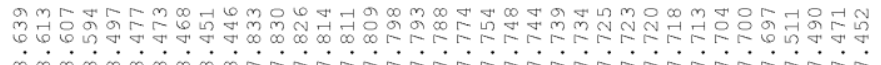

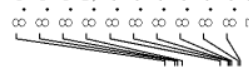
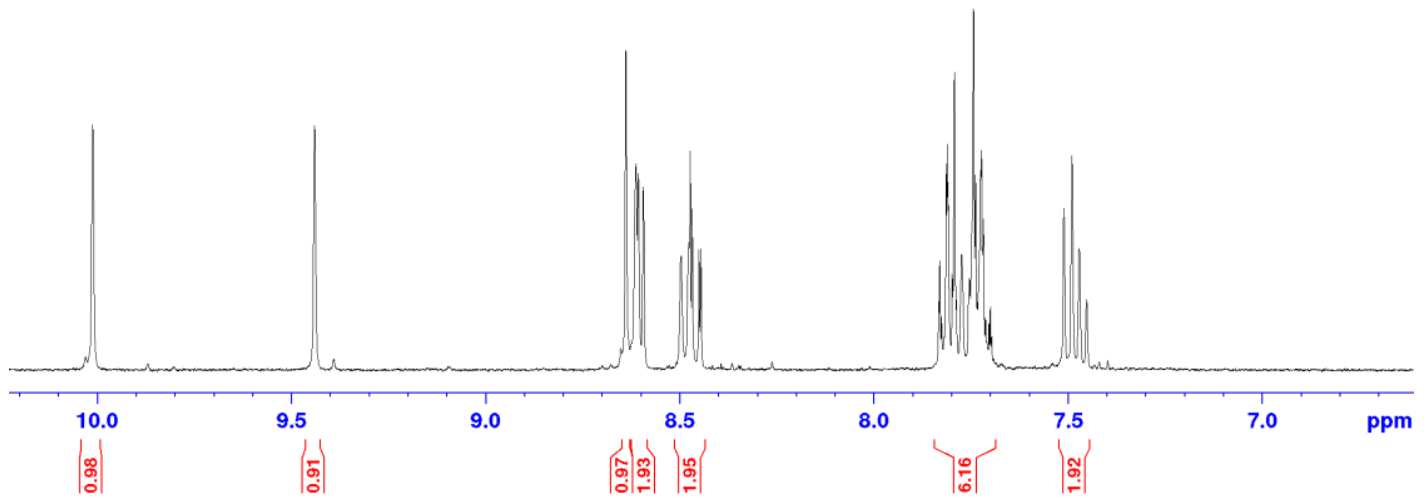

10.0
$\left|\begin{array}{c}\mid \\ 0 \\ 0\end{array}\right|$

9.5

9.0

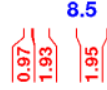

hin

Whe lhe

9

8

|

(

8

$\left|\begin{array}{l}0 \\ 0 \\ 0\end{array}\right|\left|\begin{array}{l}\mathcal{N} \\ 0 \\ \hdashline\end{array}\right|$

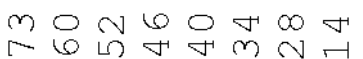

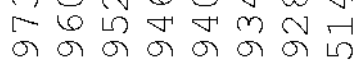
i $\cdot \dot{i} \cdot \dot{i}$

가닉

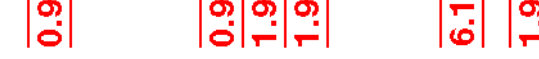


${ }^{13} \mathrm{C}\left\{{ }^{1} \mathrm{H}\right\}$ NMR (101 MHz, CD $\left.\mathrm{CN}, 298 \mathrm{~K}\right)$
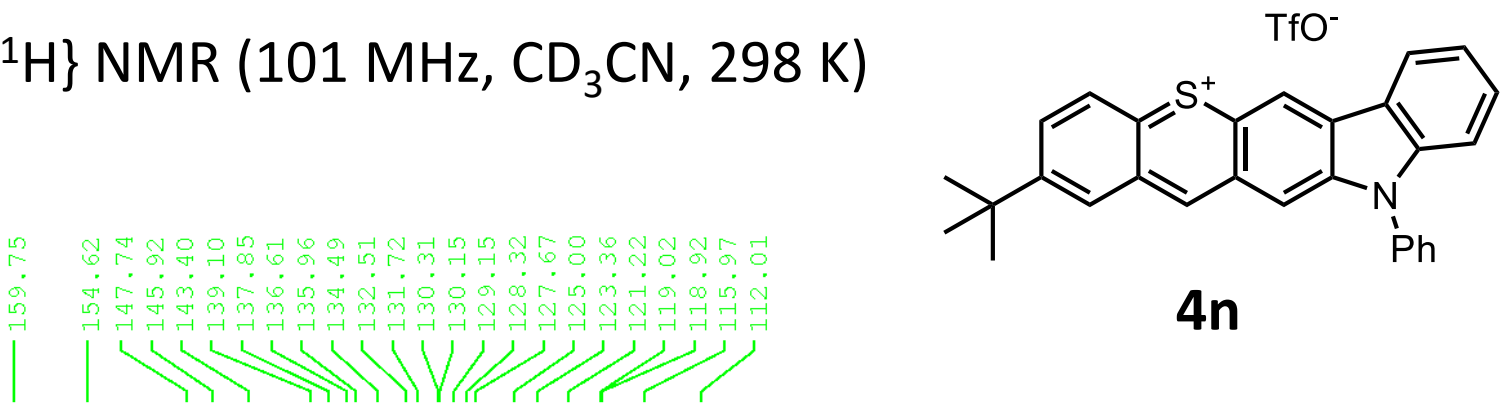

$4 n$

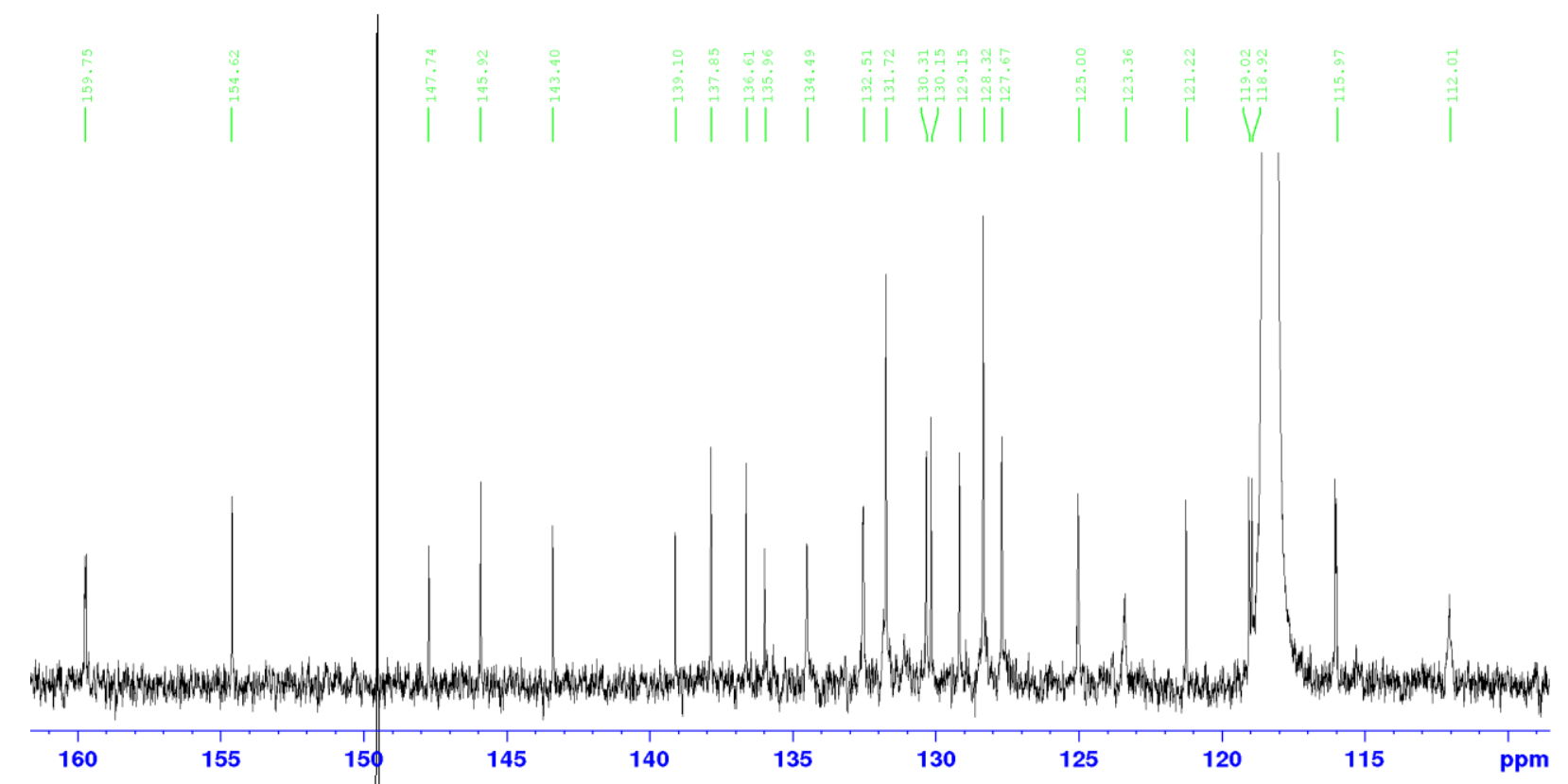

